Portland State University

PDXScholar

$1-1-2010$

\title{
Performance Measures for Managerial Decision Making: Performance Measurement Synergies in Multi-Attribute Performance Measurement Systems
}

Robert Andrew Fowke

Portland State University

Follow this and additional works at: https://pdxscholar.library.pdx.edu/open_access_etds Let us know how access to this document benefits you.

\section{Recommended Citation}

Fowke, Robert Andrew, "Performance Measures for Managerial Decision Making: Performance Measurement Synergies in Multi-Attribute Performance Measurement Systems" (2010). Dissertations and Theses. Paper 164.

https://doi.org/10.15760/etd.164

This Dissertation is brought to you for free and open access. It has been accepted for inclusion in Dissertations and Theses by an authorized administrator of PDXScholar. Please contact us if we can make this document more accessible: pdxscholar@pdx.edu. 
Performance Measures for Managerial Decision Making: Performance Measurement Synergies in Multi-Attribute Performance Measurement Systems

by

Robert Andrew Fowke

A dissertation submitted in partial fulfillment of the requirements for the degree of

\title{
Doctor of Philosophy \\ in
}

Systems Science: Business Administration

\author{
Dissertation Committee: \\ Beverly Fuller, Chair \\ Alan Raedels \\ Richard Sapp \\ Wayne W. Wakeland \\ Timothy Anderson
}

Portland State University

(C)2010 


\begin{abstract}
This research tests for correlation between corporate performance and use of financial measures, nonfinancial measures, and number of balanced scorecard categories used. Literature notes a preference for managing by nonfinancial measures because financial measures are lagging indicators, but little empirical evidence is available on the relationship between nonfinancial measures and financial performance, and few companies are found to realize the benefits of nonfinancial measurements. The balanced scorecard has been studied to find the impact of diversity of performance measures, and anecdotal improvements have been reported, but there is a paucity of empirical evidence regarding how the use of a balanced scorecard impacts organizational performance.
\end{abstract}

These issues are investigated in this research with a web based survey distributed to a sample of publicly traded companies using a systematic selection process based on randomly selected numbers generated for each 3-digit NAICS category. The dependent variable is a rank of high, medium or low performance based on 12-month rolling average stock price comparisons from January 2005 to January 2009. These averages are analyzed as a percent change for each company, with performance standardized by 3digit NAICS category to eliminate cross industry variance in performance ranking. Kruskal-Wallis one-way ANOVA is used to test for correlation.

High performers show greatest utilization of both financial and nonfinancial measures, followed by medium performers, with low performers utilizing both measures 
the least. Nonfinancial performance measures are more correlated to firm value than financial measures with the high performers' mean score for nonfinancial measures being higher than for financial measures. By contrast, medium and low performers exhibit the opposite: higher mean scores for financial measures than for nonfinancial measures $[\mathrm{p} \leq$ 0.05 for nonfinancial measures and $\mathrm{p} \leq 0.1$ for financial measures]. Correlation is found to be borderline significant $(\mathrm{p}=0.06)$ for the number of balanced scorecard categories used with high performers utilizing the highest number of categories and low performers utilizing the lowest number of categories $[p=0.009$ with inclusion of two respondents reporting no usage of balanced scorecard categories]. 


\section{AKNOWLEDGEMENTS}

I am grateful to Beverly Fuller, my chair, for her guidance in completing this dissertation and to the remaining members of my committee: Alan Raedels, Richard Sapp, Wayne W. Wakeland, Timothy Anderson; and Tom Gillpatrick who served as

proxy member. Collectively they have provided me with intellectual guidance and have been role models as researchers.

I am also grateful to Sam Adams. Without his encouragement I would not have started this project. Though he did not live to see the culmination of this research he was confident that it would be completed. He was both a friend and a mentor and is missed.

Finally, I am most grateful to my family for their support: my wife, Kelli; my children Sarah, Chelsea, Steven and Lacy; and my parents for their belief in the value of education.

With so much help from many sources, I have only myself to blame for flaws that remain. 


\section{TABLE OF CONTENTS}

PAGE

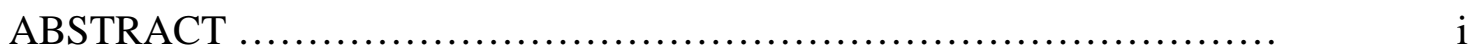

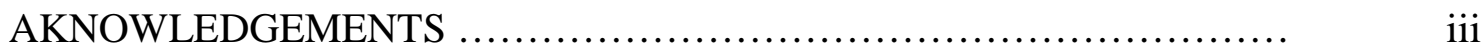

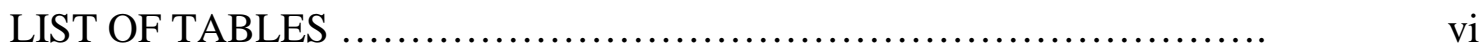

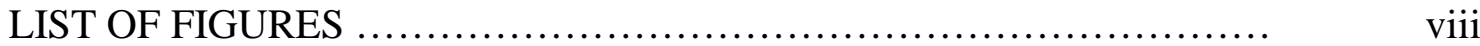

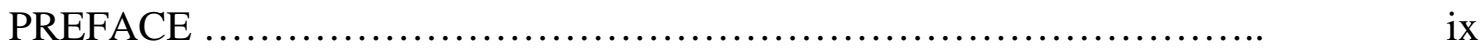

\section{CHAPTER}

I INTRODUCTION ..........................................

The Firm as a Complex Dynamic System ........................ 1

II REVIEW OF THE LITERATURE ............................

Traditional Cost Accounting ......................................

Contemporary Techniques ...................................

Agency Based Theory ....................................... 16

Contingency Based Theory .................................... 20

Activity Based Costing and Economic Value Added Measures ....... 27

Benchmarking and Key Performance Indicators ................... 30

Financial, Nonfinancial Objective - Subjective Measures:

Balanced Scorecards ......................................... 32

Performance Measurement Review Conclusions ...................

Clockspeeed ...............................................

Systems Definitions and Concepts .............................. 41

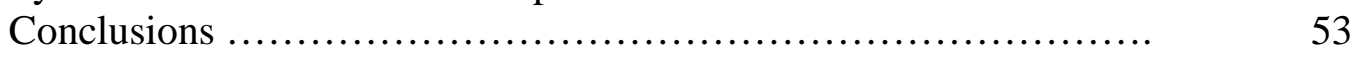

III DESCRIPTION OF THE PROBLEM .......................... 55

Questions and Hypotheses ................................... 55

IV METHODS AND TECHNIQUES ............................... 68

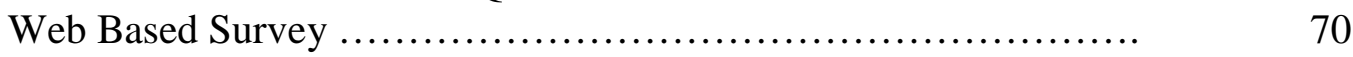

NAICS Company Definition ................................

Stock Price Histories ......................................... $\quad 73$

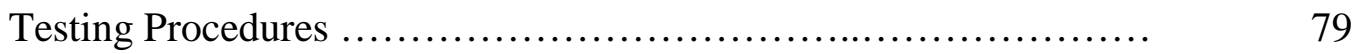

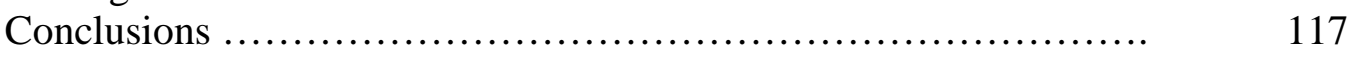

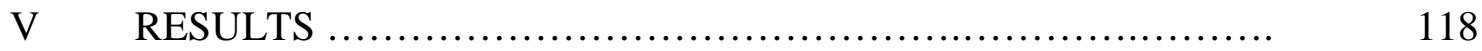

Sample Definition and Survey Setup ........................... 118

Pilot Test Observations / Conclusions / Plan ...................... $\quad 120$

Dissertation Survey Results ................................. 128

Hypothesis Testing ......................................... 132 
VI DISCUSSION ..............................................

Results and Implications ....................................

Limitations ...................................................... 161

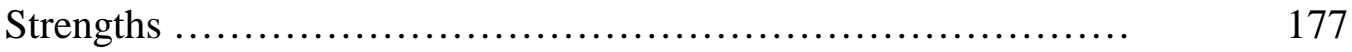

Suggestions for Further Research ............................. 180

BIBLIOGRAPY ....................................................

APPENDICES ..................................................... 191

A The Firm-A Systems Perspective (Figure 1) Detailed

Description with Citations ........................................... 191

B Proposed Dissertation Timeline ................................... 194

C Hypotheses .................................................... 195

D Consent Form Web Based Survey ................................. 196

E Questionnaire .................................................... 197

F NAICS Company Listings ...................................... 201

G NAICS Company Comparisons ................................. 207

H NAICS Codes and Companies per Code .......................... 222

I Random NAICS Category Start Number for Company Selection .... 229

J Pilot Test Email Campaign ......................................... 237

K Dissertation Survey Email Campaign ............................. 242

L Survey Results ............................................. 247

M NAICS Response Categories Data and Dependent

Variable Definition ............................................. 256

N Dependent Variable by Responding Companies Only ................ 281

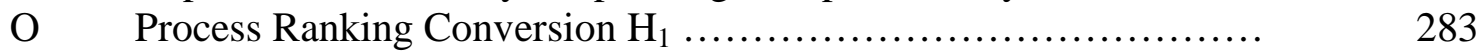

P Financial Nonfinancial $\mathrm{H}_{2}$ Kruskal-Wallis Ranked Means

Q $\quad \mathrm{H}_{3}$ Cramer's Phi ................................................ 289

R Number of Balanced Scorecard Categories Used ................... 290

S Dissertation Survey ......................................... 292 


\section{LIST OF TABLES}

\section{TABLE}

PAGE

1 Sample Industry Clockspeeds

Performance Measures Integrated Perspective ........................

NAICS 31621 Company Listings ................................

Foot Locker Stock Price Changes ............................. 75

Monthly Average Annual Stock Price Change by Company .......... 77

Company Performance Comparisons .............................. 78

Test Variables and Type of Measure

Statistical Tests of Association for Each Hypothesis ................. 80

11 Hypothetical Case - Responses by Performance Level and

Kruskal-Wallis Example Steps 3 and 4 (Identity)

Kruskal-Wallis Example Steps 1 and 2 (Priority)

27 High, Middle and Low Performer Financial and Nonfinancial

Ranked Means $\mathrm{H}_{2}$

Kruskal-Wallis Test of Ranked Means $\mathrm{H}_{2}$

106 High Clockspeed Financial and Nonfinancial Ranked Means $\mathrm{H}_{3} \ldots \ldots . .109$

31 Low Clockspeed Financial and Nonfinancial Ranked Means $\mathrm{H}_{3}$ 
33 Cramer's Phi for Medium Clockspeed Financial

Vs. Nonfinancial Ranked Use Means $\mathrm{H}_{3} \ldots \ldots \ldots \ldots \ldots \ldots \ldots \ldots . \ldots 113$

34 Cramer's Phi for Low Clockspeed Financial

Vs. Nonfinancial Ranked Use Means $\mathrm{H}_{3} \ldots \ldots \ldots \ldots \ldots \ldots \ldots \ldots . \ldots 114$

35 Number of Companies per NAICS Category Summary ................ 119

36 Pilot Test Sample Specification .................................. 120

37 Pilot Test Companies and Completion Rate ......................... 121

38 Pilot Test NAICS Category Completions .......................... 122

39 Pilot Test Financial - Nonfinancial Measures ........................... 124

$40 \quad$ Pilot Test Process Salience Ranking Responses ....................... 125

$41 \quad$ NAICS Category Responses ..................................... 130

42 Combined Survey Response Results ............................. 131

43 Dependent Variable Definition NAICS Category $211 \ldots \ldots \ldots \ldots \ldots \ldots . . . . . .133$

44 Dependent Variable Definition ...................................... 134

$45 \quad \mathrm{H}_{1}$ Kruskal-Wallis Means ....................................... 137

$46 \quad \mathrm{H}_{1 \mathrm{a}}$ Chi-Square Contingency Test Identity vs Priority .................. 141

$47 \quad \mathrm{H}_{1 \mathrm{~b}}$ Chi-Square Contingency Test Priority vs Background .............. 142

$48 \quad \mathrm{H}_{2}$ Kruskal-Wallis Means ................................................ 145

$49 \quad \mathrm{H}_{3}$ Cramer's Phi by Clockspeed ................................... 150

$50 \quad$ Balanced Scorecard Category Utilization ........................... 152

51 Balanced Scorecard Kruskal-Wallis Means ........................ 152

52 Bilateral Correlations ......................................... 167

53 Demographics of Respondents vs. Non-respondents .................. 171

$54 \quad$ Verification of Random Alternative Response Sequencing ............. 176

55 Responding Company NAICS Category Descriptions ................. 178 


\section{LIST OF FIGURES}

FIGURE

PAGE

1 The Firm-A Systems Perspective ............................... 2

2 Business Processes and Performance Measure Feedback ................. 59

3 Financial Measures vs. Nonfinancial Measures Lagged Effect ........... 61

$4 \quad \mathrm{H}_{1}$ Hypothetical Case Results .......................................... 97

$5 \quad \mathrm{H}_{2}$ Hypothetical Case Results ........................................ 107

$6 \quad \mathrm{H}_{3}$ High Clockspeed Hypothetical Case Results ........................ 115

$7 \quad \mathrm{H}_{3}$ Medium Clockspeed Hypothetical Case Results ...................... 115

$8 \quad \mathrm{H}_{3}$ Low Clockspeed Hypothetical Case Results ....................... 116

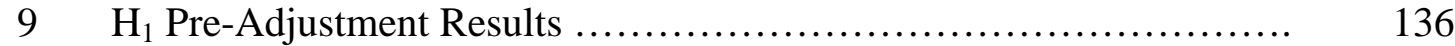

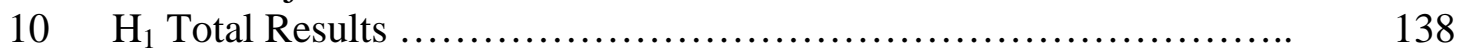

$11 \quad \mathrm{H}_{1}$ Executive Results .............................................. 139

$12 \quad \mathrm{H}_{1}$ Supervisor Results ........................................ 140

$13 \quad \mathrm{H}_{2}$ Pre-Adjustment Results ..................................... 144

$14 \quad \mathrm{H}_{2}$ Total Results ................................................ 146

$15 \quad \mathrm{H}_{2}$ Executive Results ........................................... 147

$16 \quad \mathrm{H}_{2}$ Supervisor Results ...................................... 148

$17 \quad \mathrm{H}_{4}$ Pre-Adjustment Results ...................................... 151

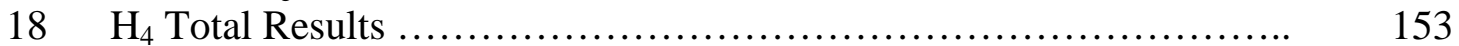

$19 \quad \mathrm{H}_{4}$ Executive \& Supervisor Results .................................. 153

20 Process Salience Usage by Performance ............................. 155

21 Ranked Means of Financial and Nonfinancial Measures Used

22 Cramer's Phi (V) Based on Clockspeed ............................... 158

23 Balanced Scorecard Utilization by Performance Category ............... 159

24 Performance Relative to Clockspeed, Asset Size and Age .............. 163

25 Correlations Relative to Position .................................... 164

26 Correlations Relative to Department ................................ 165 


\section{PREFACE}

This document is presented as it evolved with Chapters I through IV documenting proposed research as of 2008. The final two chapters document the results, and provide a discussion of the research conducted from 2008 to 2010. Therefore, Chapters I through IV are written in form referring to proposed future activities and conclusions that may or may not be realized as documented in Chapters V and VI. 


\section{CHAPTER I}

\section{INTRODUCTION}

The premise of this research project is that the firm (any business organization) is a complex dynamic system, and is a subsystem of a larger (meta) dynamic system (value chain) which operates within an even larger dynamic system (market). The purpose of this project is to show that the selection of specific subsystems of the firm for measurement (feedback) and the types of measurement used correlate to firm performance (value). To establish the context, to define the subsystems and performance measures under consideration, the firm as a complex dynamic system is now described.

\section{The Firm as a Complex Dynamic System}

The firm is a subsystem of a larger value chain system (see Figure 1 - colored boxes at bottom relative to this study -- for a detailed description see Appendix A). The value chain, comprised of upstream (Pre-Firm Value Chain) and downstream (Post-Firm Value Chain) subsystems, provides value to the metasystem environment. The firm attempts to optimize its performance based on relationships with other firms in the value chain; also by monitoring and adjusting performance of its own subsystems using a number of financial and nonfinancial performance measures. 


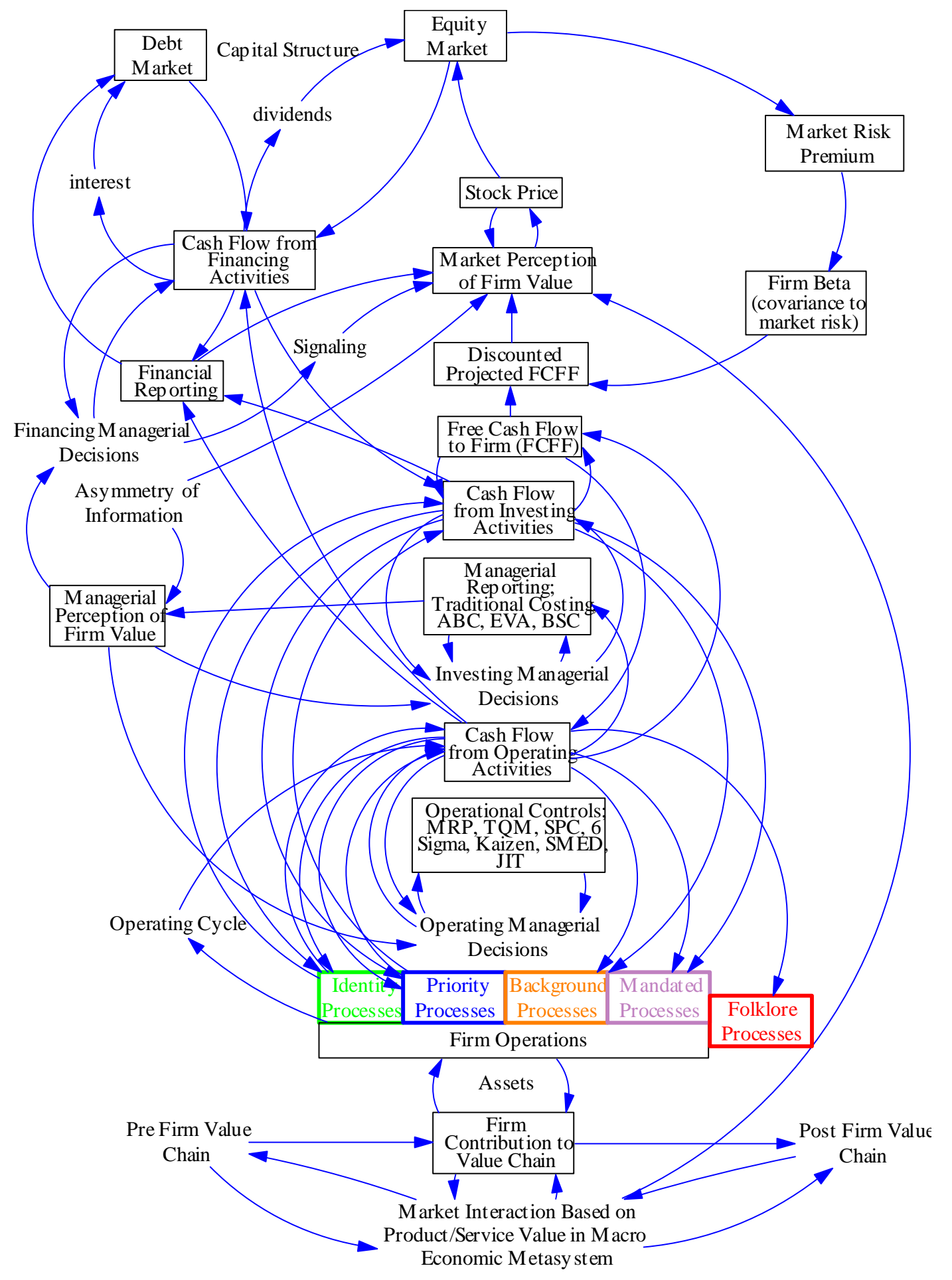

Figure 1: The Firm-A Systems Perspective 
Assets are managed by the firm using a variety of processes to generate cash flow, which creates value. For the purpose of this research, processes considered are Identity, Priority, Background and Mandated processes which are all “...coordinated activities that involve people, procedures, and technology" (Keen, 1997, p. 13). These processes are used throughout the firm system and require the use of resources (cash flow), but not all processes make a positive contribution to cash flow. Literature notes the value of nonfinancial measures (Chow \& Van der Stede, 2006), and even a preference for managing by nonfinancial measures because financial measures are after-the-fact, so using these measures does not fully address the issue of optimizing performance. Although it is true that managing by nonfinancial measures gets one "closer" to the process, this does not get to the heart of the process structure because it does not "quantify" the process structure or the "value added" components of the structure. Are there fundamental process related measures? Many companies identify Key Performance Indicators (KPI), but do the indicators truly represent "Key" processes driving profitability and success? The Balanced Scorecard proposed by Kaplan and Norton (1996) has tried to address the comprehensive nature of performance measurement requirements based on Financial, Customer, Internal Business Process, and Learning and Growth metrics, but are these measures all "Key"?

All processes used within the firm system impact management information, cash flows, market perception of value, and ultimately the stock price of the firm. This study will test the following subset of this complex dynamic system to find synergies in performance measurement systems that correlate to stock price behavior: 
- Based on Keen's perspective (1997) the Salience (Identity, Priority or Background) of the process selected for measurement impacts firm value $\left(\mathrm{H}_{1}\right)$. An identity process is one that defines the company for itself, its customers, and its investors. Priority processes are the engine of corporate effectiveness. They strongly influence how well identity processes are carried out and how a firm stands relative to its competition. Background processes are a necessary support to daily operations. Many administrative and overhead functions are background processes.

- A number of studies, including Chow and Van der Stede (2006), suggest that nonfinancial measures are better than financial measures for improving performance because financial measures are lagging indicators $\left(\mathrm{H}_{2}\right)$

- The concept of clockspeed as introduced by Fine (1998) in conjunction with the systems concept of feedback delay implies that the impact of financial vs. nonfinancial measures will be accented in high clockspeed industries $\left(\mathrm{H}_{3}\right)$. Clockspeed is a term used to designate the rate of change of product, process, or technology with high clockspeed designating rapid change.

- The balanced scorecard concept introduced by Kaplan and Norton (1996) increases firm value by assuring a balanced performance throughout the organization $\left(\mathrm{H}_{4}\right)$ 
The development and statement of these hypotheses is the topic of Chapter 3, but the designations $\left(\mathrm{H}_{1-4}\right)$ are inserted throughout Chapter 2 to note the relevance and applicability of the literature review.

A web-based survey will be used for data collection. Respondents will remain anonymous and companies will be coded for confidentiality. The comparative dependent variable is the change in month-end stock price (positive change indicates increasing value). To eliminate seasonal and year-end distortions, the comparison will be based on a rolling 12-month average month-end stock price with the comparison based on a single specific end date for all companies tested. All materials used in the study will be retained for a period of three years.

This research project will contribute to the field by providing empirical evidence of the value of using a process orientation in performance measurement development, add empirical evidence regarding the value of financial vs. nonfinancial measures, confirm the impact of clockspeed as an important contingency in performance measurement selection, and measure the impact of using a balanced scorecard. These factors will be observed over a broad range of industries as defined by North American Industry Classification System (NAICS) code.

Chapter 2 provides a review of literature relevant to this study including: a history of traditional cost management, contemporary techniques, agency theory, contingency theory, activity based and economic value added measures, financial and nonfinancial 
performance measurement, clockspeed, and a description of systems definitions and concepts. Chapter 3 develops the theoretical basis for the questions and hypotheses under consideration. Chapter 4 defines the data collection and analytic methods to be used for the study. Chapter 5 documents the results, and Chapter 6 discusses the results and provides recommendations. Appendices are included which define the proposed project timeline, the summarized hypotheses, the proposed survey respondent consent form, a preliminary questionnaire, a sampling of company listings and comparisons, and supporting material for the test. 


\section{CHAPTER II}

\section{REVIEW OF THE LITERATURE}

\section{Traditional Cost Accounting}

The history of traditional cost accounting is important because it shows how organizations have come to rely on financial measures as the primary source of information for management decisions $\left(\mathrm{H}_{2}\right)$.

Accounting, as evidenced by double-entry bookkeeping, finds its roots in

Northern Italy in the $14^{\text {th }} \& 15^{\text {th }}$ Centuries where it was formalized and published in 1494 (Davidson \& Weil, 1978, Ch 1, p. 2). The double entry method at that time was used strictly for keeping records. In 1750 the method was adapted to order costing by an English shoemaker, and in 1777 was used in textile manufacturing to record quantities and values of weaving processes external to the firm (Davidson \& Weil, Ch. 1, pp.4-5).

Accounting for textiles manufacturing in New England during the early 1800s saw a change from mercantile to industrial accounting. "Prior to this time, accounting was mercantile in that it simply established accountability for financial statement components and apportioned costs among different products and product lines" (Fleischman \& Tyson, 1997, p. 366). The introduction of the power loom (Lowell mills) in the mid-1820s in the United States resulted in a large, full-time labor force whose 
production needed close monitoring and measurement. The work force became removed from the family and "employed" managers became responsible for costs and quality (Fleischman \& Tyson, 1997, p. 367). According to Fleishman and Tyson (1997) “...accounting information was a necessity from the inception of the Lowell mills. Large, fully integrated facilities faced foreign and domestic competition in markets characterized by steadily falling market prices" (p. 368). As noted by Johnson (1981), "Managers of early textile mills could monitor employee performance with periodic cost information that compared productivity among workers in the same process at a specific time and that also compared productivity for one or more workers over several periods of time" (p. 514). This double entry system was used to obtain managerial cost information rather than to account for the costs of long-term assets.

During the time the Lowell Mills in the United States was utilizing accounting for industrial purposes, farm accounting in France was evolving to distinguish profits on cultivation from speculative profits (from holding produce after it had been raised). Long maturing investments like vineyards and woods were charged a per annum compound interest while maturing, and credited with the value of the produce obtained.

Depreciation of equipment, buildings and improvements was taken account of by writing off the cost over the expected lives of the assets, at different rates even for different parts of the same asset, according to the durability of the part (Davidson \& Weil, 1978, ch.1, p. 8). A publication in France in 1827 discusses “....depreciation and maintenance of buildings and plant, recognizes problem of pricing out materials that have been purchased at varying prices and favors a single average price for the year for each material and ... 
includes interest on capital among the expenses in ... P\&L account" (Davidson \& Weil, 1978, Ch. 1, p. 7).

With the use of accounting for managerial costing increasing, "Charles Babbage, Lucasian Professor of Mathematics in University of Cambridge, puts forward [a] case for devotion of time and attention to costing On the Economy of Machinery and Manufactures (1832)" (Davidson \& Weil, 1978, ch.1, p. 5).

Railroads as large, capital intensive institutions at this time also begin using accounting for managerial information:

As early as 1831, the managers of the $\mathrm{B} \& \mathrm{O}$ [railroad] had observed fixed and variable cost behaviour [sic] and had understood certain 'cost drivers' pertaining to passenger and freight service. They even used measures such as freight ton-miles as part of their management control. From its inception, the B\&O published various operating and comparative statistics such as total passengers and miles run, as well as tons carried and cost-per ton (Heier, 2000, p. 216).

In 1850 a 500-page treatise, Railroad Economy, published by Dionysius Lardner discusses the development of railroads and their operations in the United States, England and Europe. This treatise includes statistics to explain operations and explains factors that impacts railroad expenses (Heier, 2000, pp. 217-218).

It is at this time that agency theory issues begin to be recognized: 
Beginning in the mid-1850s, Henry Varnum Poor, publisher of Poor's American Railroad Journal, encouraged railroads to create and publish statistics such as tonmiles and revenue-miles so that the public could better evaluate their performance. Poor felt this was necessary because so many rail managers were not owners, and conflicts of interest between managerial motives and owners' expectations began to arise. The agency problems inherent with absentee ownership were just beginning to emerge (Heier, 2000, p. 218).

By 1869, operating statistics and cost data are being presented in annual reports, and standardized cost data are used to set shipping tariffs for freight and passenger service (Heier, 2000, p. 215). Though elaborate cost reporting and estimation schemes are used by the $1880 \mathrm{~s}$, overhead and capital costs are not considered in cost reporting (Kaplan, 1984a, pp. 392-393).

By 1900 the industrial uses of accounting are increasingly recognized by business people because of the increasing scale and complexity of business:

In the iron industry, for example, the size of business and the degree of integration had ceased to be limited by the availability of water power. The coal mining industry had developed similarly; the sinking of deep shafts, construction of galleries, ventilation and pumping equipment, and transport all called for large capital investment. The textile industries had been transformed by the introduction of power machinery. Thus the importance of buildings, plant, equipment, and supervision, i.e., of overhead costs, had rapidly increased in relation to the prime costs of materials and labor. Larger bodies of labor had to be paid and controlled, complex processes to be organized and administered. Moreover, the development of railways, with their enormous volume of fixed equipment, had brought the problem of overhead to the fore (Davidson \& Weil, 1978, ch.1, p. 10). 
Standard costing appears in the early 1900s and evolves to the point of being an established practice by 1918 (Kaplan, 1984a, p. 394) and by 1919 “...the process of attaching overhead costs to units of product had been brought more or less to completion" (Davidson \& Weil, 1978, Ch. 1, p. 17).

After 1925 a subtle change occurred in the information used by managers to direct the affairs of complex hierarchies. Until the 1920s, managers invariably relied on information about the underlying processes, transactions, and events that produce financial numbers. By the 1960s and 1970s, however, managers commonly relied on the financial numbers alone (Johnson \& Kaplan, 1987, pp. 125-126).

The end result of this reliance on "traditional cost accounting" financial measurement system is that managers rely on accounting numbers $\left(\mathrm{H}_{2}\right)$ and not on the processes behind the numbers $\left(\mathrm{H}_{1}\right)$.

\section{Contemporary Techniques}

A number of different contemporary techniques have been developed and studied. Contemporary techniques are important because they have been designed to improve organizational performance and value beyond that possible using traditional cost accounting alone. By the 1980s and 1990s increasing competition from globalization and the focus on the customer in the value chain highlight the need for alternative performance measurement systems. Heskett, Jones, Loveman, Sasser, and Schlesinger (1994) describe the concept of customer satisfaction driving profitability as follows: 
The links in the chain... are as follows: Profit and growth are stimulated primarily by customer loyalty. Loyalty is a direct result of customer satisfaction.

Satisfaction is largely influenced by the value of services provided to customers. Value is created by satisfied, loyal and productive employees. Employee satisfaction, in turn, results primarily from high-quality support services and policies that enable employees to deliver results to customers (pp. 164-165).

Simons (1991) defines management control systems as “...the formalized routines and procedures that use information to maintain or alter patterns in organizational activity" noting that “...these systems include formalized information-based processes for planning, budgeting, cost control, environmental scanning, competitor analysis, performance evaluation, resource allocation, and employee rewards" (p. 49). The need for changes to traditional management accounting is noted by Kaplan (1984a) who states that management accounting “...cannot exist as a separate discipline, developing its own set of procedures and measurement systems and applying these universally to all firms without regard to the underlying values, goals, and strategies of the particular firms", but must "... serve the strategic objectives of the firm" (p. 414). Bromwich (1990) considers "strategic management accounting" and the importance that accountants consider the cost structure of not only the firm, but of all enterprises in the relevant market and even potential entrants. Costs cannot be considered separate from demand; therefore the accountant needs to consider whether the firm's cost structure allows its strategy to be sustainable in the face of potential entry. This second view considers demand and cost factors and their linkage, while highlighting the importance of fixed costs and sunk costs. Hemmer (1996) comments that to reinforce the long-term aspects of actions, “...modern management accounting incorporates, as a significant element, nonfinancial (and from an 
accounting standpoint, nontraditional) measures of customer satisfaction and defects" (p. 87). He further notes that little evidence has been found to determine under which conditions alternative designs perform well.

Chenhall and Langfield-Smith (1998) define a variety of combinations of strategies, management techniques and accounting practices used to pursue competitive advantage. The two strategic priorities noted are low price or differentiation as developed by Porter. Management techniques include improving existing processes, quality systems, manufacturing systems innovations, integrating systems (across functions and with suppliers and customers), team based structures, and human resource management policies. Management accounting practices include traditional techniques and contemporary techniques including benchmarking, activity-based costing, balanced performance measures, team-based performance measures, employee based measures and strategic planning (p. 244). They state, “...in high performing companies, these variables may act in combination to provide synergies or complementarities to enhance organizational performance" (p. 245). In a review of 320 different studies of performance, Capon, Farley and Hoenig (1990) note that “... results hint at the presence of strong interactive effects among variables (p. 1157)". They further note:

There may be synergies (positive and negative) leading to various optimal combinations of factor inputs. Work on interaction of causal factors is badly needed if the goal of analysis is to move towards optimal allocation of resources among controllable variables (pp. 1158-1159). 
Lee, Kwak and Han (1995) propose an Analytic Hierarchical Model with four to seven levels of performance measurement consideration. The operational level (lowest level) uses quality, cycle time, delivery, cost, inventory turnover, asset turnover and other similar performance measures. The middle level uses market share, customer satisfaction productivity, ROI, and profitability measures. The top level (executive) uses financial and nonfinancial performance measures. The hierarchy can be adjusted as the company or environment changes.

Kaplan and Norton (1996) summarize the "contemporary" issues facing organizations, noting that product life cycles continue to shrink and changing technological platforms assure that competitive advantage in one generation of a product's life is no guarantee of product leadership in the next (p. 5). They state that the financial-reporting process continues to be based on traditional accounting while the organization is trying to forge linkages and strategic alliances with external parties (pp. 67), and the financial accounting model needs to be “...expanded to incorporate the valuation of a company's intangible and intellectual assets, such as high-quality products and services, motivated and skilled employees responsive and predictable internal processes, and satisfied and loyal customers" (p. 7). They further note that financial measures have a short-term view and are based on past events, which has traditionally been sufficient since investments in long-term capabilities and customer relationships are not critical for success. The Balanced Scorecard proposed by Kaplan and Norton is intended to articulate the theory of the business and "...should be based on a series of cause-and-effect relationships derived from the strategy, including estimates of the 
response times and magnitudes of the linkages among the scorecard measures" (p. 17).

"A good Balanced Scorecard should have an appropriate mix of outcomes (lagging indicators) and performance drivers (leading indicators) of the business unit's strategy" (p. 32).

Ittner and Larcker (1998) examine three measurement trends: 1) economic value measures, 2) nonfinancial performance measures and the balanced scorecard, and 3) performance measurement initiatives in government agencies. They state most economic theories support the contention that "...performance measurement and reward systems should incorporate any financial or nonfinancial measure that provides incremental information on managerial effort (subject to its cost)" (p. 206) while at the same time commenting on the insufficiency of current measurement systems: a 1996 survey by the Institute of Management Accounting found that only $15 \%$ of the respondents' measurement systems supported top managements' business objectives well while $43 \%$ were less than adequate or poor (p. 205). It would seem that either additional measurement systems are needed, or measurement system targeting needs adjustment.

Otley (1999) considers a framework structured around 5 issues: 1) objectives, 2) strategies and plans for their attainment, 3) target-setting, 4) incentive and reward structures, and 5) feedback loops. These five issues are tested against three major organizational control systems: budgeting, economic value added, and balanced scorecard. Otley's conclusion is that “...management accounting and other performance measurement practices need to be evaluated not just from an economic perspective, but 
from a social, behavioral and managerial perspective, within an overall organizational context" (p. 381).

Much has been written about performance measurement relative to different subsystems within the firm. Contemporary techniques have been described that include a variety of financial and nonfinancial measures $\left(\mathrm{H}_{2}\right)$ as well as balanced scorecard $\left(\mathrm{H}_{4}\right)$ systems.

\section{Agency Based Theory}

As noted in the history of traditional cost accounting, by the mid-1850s "The agency problems inherent with absentee ownership were just beginning to emerge (Heier, 2000, p. 218). Agency theory has been well studied and is important because it attempts to find ways to improve employee performance and thereby improve organization performance.

Agency theory deals with the problems of creating a contract governing an exchange between individuals who have divergent interest. In the employment relationship, the basic agency problem is characterized in terms of structuring monitoring and compensation systems so that they will induce self-interested, utility-maximizing, risk-and-effort-averse agents (managers who want to maximize their compensation and minimize their effort expenditures) to act on the behalf of principals - or owners - who want to increase the value and performance of their firms (Bloom \& Milkovich, 1998, p. 283). 
Agency theory assumes “...that both parties are (1) rational and (2) self-interested, and that the agent is (3) both effort- and risk-averse" (Bloom \& Milkovich, 1984, p. 284). Bloom and Milkovich find that higher-risk firms that rely on incentive pay exhibit poorer performance than higher-risk firms that do not emphasize incentive pay, adding evidence to the findings by Pearce, Stevenson and Perry (1985) who find that merit pay does not improve organizational performance. Contrary to the findings of Bloom and Milkovich (1984), and Pearce, et al. (1985), Banker, Potter and Srinivasan (2000) find that both nonfinancial and financial performance improves following the implementation of an incentive plan that includes nonfinancial performance measures.

Feltham and Xie (1994) look at economic trade-offs in multiple performance measure systems, specifically noting, “...performance measures frequently are incomplete or imperfect representations of the economic consequences of the manager's actions" (p. 429). The noisiness of the measure, due to uncontrollable events, and the congruence of the measure are considered, as is the value of additional performance measures to reduce risks and non-congruity. Chenhall (1997) finds stronger performance when manufacturing performance measures are used as part of managerial evaluation (p. 200).

Abernethy and Brownell (1997) show that where task uncertainty is highest (well established techniques for performing tasks is low) and number of exceptions (degree of variety in the tasks) is high, reliance on personnel forms of control has a positive effect on performance. 
Datar, Kulp and Lambert (2001) review multi-dimensional compensation contracts and performance weighting systems. They find that “...the weight assigned to a performance measure is not simply a function of its own 'congruence' with the outcome, but also on how it interacts with the other variables in the contract" (p. 88). Bushman, Indjejikian and Smith (1995) use an agency model to show that the use of aggregate performance measures relative to more localized performance measures is an increasing function of intrafirm interdependencies.

Ittner, Larcker and Meyer (2003) in a review of subjectivity and the weighting of performance measures in a Balanced Scorecard relative to compensation systems find that:

... subjectivity in the scorecard plan allowed superiors to reduce the "balance" in bonus awards by placing most of the weight on financial measures, to incorporate factors other than the scorecard measures in performance evaluations, to change evaluation criteria from quarter to quarter, to ignore measures that were predictive of future financial performance, and to weight measures that were not predictive of desired results (p. 725).

The conclusion of their study is “...that greater weight will be placed on quantitative measures than on qualitative measures, and that greater weight will be placed on measures that are based on aggregations of multiple indicators and performance relative to targets than on other measures" (Ittner, Larker \& Meyer, 2003, p. 732). The findings of Ittner, Larker and Meyer are duplicated by Moers (2005) who states "Performance 
measure diversity leads to more lenient performance ratings and less differentiation among employees... subjectivity leads to bias in performance evaluation...if more subjectivity is used in evaluating and rewarding employees, superiors give higher performance ratings and compress these ratings (p. 79). Baker, Gibbons and Murphy (1994) review explicit and implicit contracts and find in some circumstances objective and subjective measures are complements, but objective weights that do not distort incentives will always be preferred to subjective weights.

Said, HassabElnaby and Wier (2003) examine the implications of nonfinancial performance measures included in compensation contracts on current and future performance. They note that contextual factors, environmental factors, and strategic plans vary across firms and, in turn, adopting appropriate nonfinancial measures determines the performance consequences of such measures. They conclude that firms that use a combination of financial and nonfinancial performance measures have higher returns on assets and higher levels of market returns (p. 193).

Ramachandran (2004) finds that the usefulness of accounting measures in compensation contracts is “...dependent on how accurately they provide information about the underlying managerial actions and to what extent their informativeness is clouded by the discretion managers have in reporting accounting numbers" (p. 61).

Chow and Van Der Stede (2006) conclude that financial, quantitative nonfinancial and subjective measurements each have their own strengths and weakness. Each has 
different impacts on employee actions like risk taking, innovation, emphasis on long- and short-term view, and tendency to game the performance evaluation system. Nonfinancial measures encourage risk taking and innovation and limit gamesmanship. There is no significant difference between financial and nonfinancial measurements in contributing to operational and strategic decision-making or ability to align intra and interdepartmental objectives.

Information economics and agency theory research offers the potential for a rigorous, analytic theory of management accounting, rooted in the utility and profitmaximizing behavior of neo-classical economics, as well as in the more recent analytical tools of statistical decision theory and noncooperative multiperson game theory (Kaplan, 1984a, p. 404).

Agency theory addresses the human subcomponent of the firm system in an attempt to improve organization performance, is a component of balanced scorecards $\left(\mathrm{H}_{4}\right)$, and includes financial and nonfinancial measurements $\left(\mathrm{H}_{2}\right)$.

\section{Contingency Based Theory}

Contingency based theory is important because specific measures will have different impacts on organizational performance depending on the conditions under which they are applied, and contingency theory attempts to define which measurements are best under which conditions.

The term contingency means that something is true only under specified conditions. As such there is no 'contingency theory,' rather a variety of theories may be used to explain and predict the conditions under which particular MCS 
[Management Control Systems] will be found or where they will be associated with enhanced performance (Chenhall, 2003, p. 157).

Chenhall (2003) reviews contingency-based research relative to Management Control Systems (MCS) and the way it has evolved to address the contemporary environment with Just in Time, Total Quality Management and Flexible Manufacturing as dimensions of context (p. 141), noting that contextual variables include the “...external environment, technology (traditional and contemporary), organizational structure, size, strategy and national culture" (p. 128).

According to Chenhall (2003), Agency Theory from economics attempts to maximize the benefit to the principal (employer) with incentive schemes to get commitment from the agents (employees) who are "...assumed to be self-serving and opportunistic" (p. 157) to achieve goals defined by the principal. "Agency theories have been criticized for not considering the context in which principals and agents contract and for not investigating the trade-offs with other elements of control systems" (p. 157). Psychology has attempted to identify “...individual characteristics such as personality or cognitive style affect the way individuals react and respond to different aspects of MCS" (p. 158). Behavioral economics provides a descriptive base for economic research by looking at empirical evidence. Contingency-based research has been criticized for its reliance on "traditional, functionalist theories" without applying more "interpretive and critical views" (pp. 158-159). Alternative approaches, derived from sociological literature, have been used to provide an interpretive and critical focus. "A strength of 
'alternative' approaches is that they show the potential conflict between individuals and groups and how MCS may be implicated in these struggles" (p. 159). Davis, Schoorman and Donaldson (1997) look at the differences between agent based [economic] and Stewardship [psychology and sociology] based theories of management. They advise “...assumptions made in agency theory about individualistic utility motivations resulting in principal-agent interest divergence may not hold for all managers" (p. 20), and conclude "Managers whose needs are based on growth, achievement, and selfactualization and who are intrinsically motivated may gain greater utility by accomplishing organizational rather than personal agendas" (p. 43).

Drazin and Van de Ven (1985) review the "fit" of different approaches to structural contingency theory stating, "The key concept in a contingent proposition is fit, and the definition of fit that is adopted is central to the development of the theory, to the collection of data, and to the statistical analysis of the proposition" (p. 515). The premise of the models reviewed is that "...context and structure must somehow fit together if the organization is to perform well" (p. 514). In this study, three approaches are studied:

- Selection Approach originally hypothesized that organizational context (environment, technology, or size) is related to structure (centralization, formalization, or complexity). More recently, “...natural selection and managerial selection perspectives have surfaced and provide some justification for viewing fit as a basic assumption underlying congruence propositions between organizational context and structure and process" (Drazin \&Van de Ven, 1985, p. 
516). This approach implies a relationship to performance since performance drives the "natural selection" process. This approach also “... takes into account macro- and micro- levels of the organization design" (Drazin \&Van de Ven, 1985, pp. 516-517).

- Interaction Approach explains organizational performance from the interaction of organizational structure and context “...based on Ashby’s (1956) concept of requisite variety, in which organizational adaptability is enhanced when the degree of complexity present in the environment is reflected in the structure of the organization" (Drazin \&Van de Ven, 1985, p. 517).

- Systems Approach eliminates the constraint present in both Selection and Interaction approaches where single contextual and structural factor pairs are studied relative to performance. The systems approach promotes “...the understanding of context-structure performance relationships...by addressing simultaneously the many contingencies, structural alternatives, and performance criteria that must be considered holistically to understand organization design" (Drazin \&Van de Ven, 1985, p. 519). The trade-offs that result from looking at single pairs of factors with conflicts is addressed in the systems approach by using a pattern analysis for the interactions of multiple contingencies and structural patterns with a view to internal consistency (Drazin \&Van de Ven, 1985, pp 521$522)$. 
Results from the Van Drazin and Van de Ven (1985) review find no empirical evidence to support the interaction approach, but does find support for the selection and systems approaches.

Chow, Shields and Chan (1991) look at the differences in Asian and Western manufacturing quality and consider whether these differences are due to “...firms' management controls, the national culture [collectivism vs. individualism] of their employees, or the interaction of these two factors" (pp. $209-210$ ). They find that the “...results are consistent with cultural individualism and management controls having independent, but not interactive, effects on manufacturing performance" (p. 209).

Chenhall and Langfield-Smith (1998) examine how different combinations of management techniques and accounting practices affect organizational performance based on differing (low cost or differentiation) strategic priorities (p. 243). They find that different managerial mind sets underlying differentiation and low price strategies may influence preferences for particular management accounting practices and the variables may act in combination to provide synergies or complementarities to enhance organizational performance (p. 245).

Chapman (1997) looks at typical contingent variables such as technology, environment, and strategy and suggests they might be seen as measuring the level of complexity facing organizations, though uncertainty is the driver for the organization's information processing requirements. "Uncertainty is caused not only by the interaction 
of a number of external contextual factors, but critically also by factors such as the level of organizational knowledge and understanding of how these impact on internal processes" (p. 201).

Ittner and Larcker (1998) note that the link between nonfinancial measures such as customer satisfaction and subsequent accounting and stock market performance vary across industries. "Similarly, the use and performance consequences of these measures appear to be affected by organizational strategies and the structural and environmental factors confronting the organization" and a need for evidence on the "...contingency variables affecting the predictive ability, adoption and performance consequences of various nonfinancial measures and balanced scorecards" (p. 224).

Davila (2000) investigates the relationship between project uncertainty, product strategy and management control systems in the Research and Design environment finding that cost and design information improve performance while time information decreases performance.

Amir and Lev (1996) find that financial information is largely irrelevant in fast changing technology based industries, while Davila (2000) notes "Current emphasis on first mover advantages, fast product introductions, more demanding product functionality, and shortening life cycles has put greater pressure on new product development" (p. 383). Evidence is found that project managers rely on nonfinancial measures much more than they do on financial ones due to an "....implicit assumption 
that good performance in nonfinancials will drive good financial performance (p. 404). The study provides evidence supporting a contingency theory of management control systems with alignment between the design and use of these systems and product strategy significantly related to performance (p. 404).

Bouwens and Abernethy (2000) look at the management accounting system design for firms that pursue customization as a strategic priority finding that customization affects the management accounting system by interdependencies rather than directly.

Lillis (2002) finds combined emphasis on manufacturing efficiency and customer responsiveness is "problematic", while joint emphasis on quality and efficiency is relatively easily managed.

Moores and Yuen (2001) look at management accounting systems relative to lifecycles, finding that “...changes in an organization follow a predictable pattern across discrete stages of development over time..." (p. 353).

"Research is just starting to be published identifying contingencies surrounding the design and implementation of $\mathrm{ABC} / \mathrm{ABM}$ [Activity Based Costing / Activity Based Management]...[and] there is very little published contingency work on balanced scorecards, target costing, life cycle costing, the broad array of nonfinancial performance indicators including those related to human resource management initiatives" (Chenhall, 2003, p. 130). 
Contingency theory assumes that performance measurement optimization will be dependent on the circumstances under which the system is applied. Clockspeed is one contingency considered $\left(\mathrm{H}_{3}\right)$, and the impact of financial and nonfinancial measures under different contingencies have been reviewed $\left(\mathrm{H}_{2}\right)$,

\section{$\underline{\text { Activity Based Costing and Economic Value Added Measures }}$}

The traditional and contemporary contingency-based strategies noted above are supplemented by more targeted financial measurement systems. In addition to traditional financial "costing" systems, Activity Based Costing (ABC) and Economic Value Added (EVA) financial measurement systems are also proposed. These two measures are important to note since they are widely utilized and are attempts to improve the value of financial measurement systems.

As noted by Thyssen, Israelsen, \& Jorgensen (2006), the ABC accounting methodology introduced by Kaplan (1983, 1984a, 1984b) evolved from traditional cost accounting to provide better performance measurement in the contemporary environment. Kaplan (1983) notes that cultural and environmental differences have been considered to be responsible for the superior manufacturing performance of Japanese firms (p. 687), but argues that "Traditional cost accounting systems based on an assumption of long production runs of a standard product, with unchanging characteristics and specifications...[are]...not relevant in this new [contemporary] environment" (pp. 688- 
689). Kaplan further advises that accountants need to develop measurements to address quality, inventory, productivity, and new product technologies in the "new" environment. Use of external reporting systems for internal reporting and evaluation systems result in distortions of economic performance (Kaplan, 1984b, p. 99). Cooper and Kaplan (1988) proposed activity-based costing as an alternative to typical cost accounting systems to reduce distortions in the cost data. The theory is that all of a company's activities that support production and delivery should be considered product costs, and should be split apart and traced to individual products or product families (pp. 96-97). Cooper and Kaplan (1991) advise that to use ABC effectively “...requires a conceptual break from traditional cost accounting systems...”(p. 130). They further highlight the advantage of the $\mathrm{ABC}$ analysis in its ability to view the business in different ways, “... by product or group of similar products, by individual customer or client group, or by distribution channel...." (p. 131). One main advantage to an ABC analysis is the information allowing reduction of resource consumption (p. 135). The implementation and uses of ABC continue to be described and studied (Roztocki, 2001b; Roztocki, 2001c; Roztocki, 2003; Roztocki, \& Schultz, 2003; Roztocki, Valenzuela, Porter, Monk, \& Needy, 1999; Roztocki, \& Weistroffer, 2004a; Roztocki, \& Weistroffer, 2004b; Roztocki, \& Weistroffer, 2005; Thyssen, Israelsen, \& Jorgensen, 2006). Anderson and Young, (1999) commenting on (Chenhall \& Langfield-Smith, 1998) state that “...recent empirical evidence supports the view that $\mathrm{ABC}$ effectiveness depends on organizational and technical factors" and that "... what defines an effective ABC system: whether ABC data are used in product cost reduction or process improvement; and, whether ABC data 
are more accurate than data from traditional cost system" (p. 526). They find that the outcome of $\mathrm{ABC}$ implementation is influenced by the contextual setting (p. 555).

The EVA concept trademarked by Stewart (1991) has its proponents (Keen, 1997; Roztocki, \& Needy, 1999b) due to its more accurate representation of "actual costs". Ittner and Larcker (1998) recognize that “...traditional accounting measures such as earnings per share and return on investment are the most common performance measures..." but "...they have been criticized for not taking into consideration the cost of capital and for being unduly influenced by external reporting rules (p. 209). These weaknesses are being addressed by economic value measures, founded on residual income and internal rate of return concepts (p. 209). Further works cite the advantages of a combined ABC-EVA implementation (Roztocki, 2000a; Roztocki, 2000b; Roztocki, 2000c; Roztocki, 2001a; Roztocki, 2001d; Roztocki, \& Needy, 1998; Roztocki, \& Needy, 1999a; Roztocki, \& Needy, 1999c; Roztocki, \& Needy, 2000). Though there are proponents of EVA, Biddle, Bowen and Wallace (1997) find:

There is little evidence to support the Stern Stewart claim that EVA is superior to earnings in its association with stock returns or firm values. In no case does EVA significantly outperform EBEI [earnings before extraordinary items - note that earnings is not a cash flow] in tests of relative information content. On the contrary, in most cases the evidences suggest that earnings outperforms EVA" (pp. 331-332). 
The claim is made that both $\mathrm{ABC}$ and EVA positively impact the quality of financial information usefulness and improve firm performance. This may have an impact on the relative value of financial and nonfinancial measures $\left(\mathrm{H}_{2}\right)$.

\section{Benchmarking and Key Performance Indicators}

Benchmarking and Key Performance Indicators (KPI) are important concepts to review since the performance measures utilized help organizations choose best practices to improve their performance.

El-Mashaleh, Minching and O’Brien (2007) propose benchmarking using DEA (data envelope analysis) to foster trade-off analyses among various performance metrics. They state, “...a firm's cost performance may improve, but schedule performance declines. How can one determine whether this trade-off is truly desirable? Is the overall performance of the firm better..." (p. 12)? These questions are addressed by DEA which uses mathematical linear programming to form an envelopment surface (efficient frontier) with

...three inherent powerful features. First, it has the ability to incorporate multiple inputs and multiple outputs-particularly when it is used in conjunction with linear programming. Linear programming can handle large numbers of variables and relations (constraints). Second, DEA has no a priori assumptions. There is no need to assign weights to the different inputs and outputs. The weights are derived directly from the data, freeing the user from arbitrary, subjective weightings. Third, the measurement units of the different inputs and outputs need not be congruent. Some may involve the number of persons, areas of floor space, money expended, etc. The various scaling adjustments required for graphical purposes do not affect the relationships among the variables themselves... (p. 14). 
Haponova, Al-Jibouri and Reymen (2006) look for KPI in benchmarking. They note "Many of the indicators are also focused on product and not on the process. There are few existing indicators that inform stakeholders about how well their process is going during the various stages" (p. 1). Moor and Smits (2002) review KPI in a "Community of Practice". Different methods they look at include:

- Human Resources Accounting (HRA) Method which measures the added value of the members of an organization by aggregating salary expenses ( $\mathrm{p}$. 20).

- EVA Method which calculates the net revenues minus operational expenses, taxes, and interest (p. 20).

- $B S C$ Method which is a management tool that aligns measures with key strategies, tracks progress, and assigns accountability (p. 20).

- Intellectual Capital (IC) Method which monitors the intangible resources in an organization. The IC method first distinguishes between Financial Capital (monetary resources) and Intellectual Capital (intangible resources) which is further subdivided into Human Capital (intangible resources possessed by individuals) and Structural Capital (intangible resources available to the organization). The IC approach takes the strategy of the 
organization as the basis. Finally, relevant categories, critical success factors and related indicators of IC are identified and later aggregated (p. 21).

The performance measures utilized for benchmarking and KPI relate to all hypotheses under consideration in this present study.

\section{Financial, Nonfinancial Objective - Subjective Measures: Balanced Scorecards}

Financial, nonfinancial objective, nonfinancial subjective $\left(\mathrm{H}_{2}\right)$ and balanced scorecards $\left(\mathrm{H}_{4}\right)$ have been mentioned in previous sections of this chapter. There are, however, additional studies and concepts relating to these measures that do not easily fit into the prior categories and are worth mentioning here.

“... measures, such as product innovation, product leadership, employee skills and morale, or customer loyalty, may be much better indicators of future profitability than annual profits" (Kaplan, 1984a, p. 413). According to Abernethy and Brownell (1997) "Considerable attention is now being directed towards understanding the role of accounting controls in other settings where the characteristics of the tasks undertaken bring into question the suitability of conventional, accounting-based controls and raise the prospect that for effective control, organizations will need to design their control systems around a variety of non-accounting controls" (p. 233). Abernethy and Lillis (1995) find that "The use of efficiency-based performance measures in manufacturing declines as a firm's commitments to flexibility increases" (p. 249). Perera, Harrison and 
Poole (1997) provide empirical evidence to support that of Abernethy and Lillis (1991) when they note that "...changes in manufacturing strategies to emphasize quality, flexibility, dependability and low cost should be accompanied by changes in formal performance measurement systems to place greater emphasis on nonfinancial (operations-based) measures" (p. 569). They also provide empirical evidence of the increased use of nonfinancial performance measures by firms pursuing a customerfocused manufacturing strategy (pp. 568 - 569).

The BSC proposed by Kaplan and Norton (1996) is intended to articulate the theory of the business and "...should be based on a series of cause-and-effect relationships derived from the strategy, including estimates of the response times and magnitudes of the linkages among the scorecard measures" (p. 17). "A good Balanced Scorecard should have an appropriate mix of outcomes (lagging indicators) and performance drivers (leading indicators) of the business unit's strategy" (p. 32). Neely et al. (1997) presents a framework with 22 recommendations for designing and auditing BSC performance measures. In this framework, subjective measures are not included as confirmed in Item 22 "Performance measures should be objective - not based on opinion" (p. 1137). Neely et al. (2000) point out that though the process of designing a measurement system is valuable to managers, the key to a successful measurement system is implementation. “The real challenges for managers come once they have developed their robust measurement system, for then they must implement the measures. As soon as they seek to do so they encounter fear, politics and subversion" (p. 1142). Even successfully implemented systems tend to become overly complex with new measures added while 
obsolete measures are retained. "Hence the importance of research into these four interlinked themes of measurement system design, implementation, use and ongoing management, and the people, processes, infrastructure and culture issues associated with them" (p. 1142).

Nagar and Rajan, (2001) find that

...both financial quality measures, such as external product failure costs, and nonfinancial quality measures, such as defect rates and on-time deliveries, are significantly associated with future sales. One potential reason that both sets of measures are leading sales indicators is that external failure costs are customerinitiated measures, while defect rates and on-time deliveries are firm-initiated. These results imply that a collection of financial and nonfinancial quality measures best captures the effect of quality on future revenues" (p. 512).

Van der Stede, Chow and Lin (2006) examine the distinction between objective and subjective performance measures stating that "Prior empirical research has typically only differentiated between financial and nonfinancial performance measures" (p. 186). They find that performance measurement diversity is beneficial, though combining quality-based strategies with extensive use of objective nonfinancial measures is not associated with higher performance (p. 186). 


\section{Performance Measurement Review Conclusions}

Performance measurement is intended to help organizations improve their performance, includes a number of different tools that exhibit varying degrees of success under different contingencies, and can be viewed from a variety of different perspectives. This concludes the review of literature relating to performance measurement. The following two sections review clockspeed and systems literature since they are integral concepts in this study.

\section{Clockspeed}

Clockspeed $\left(\mathrm{H}_{3}\right)$ is important because it is a dominant factor in our rapidly changing global marketplace. Fine (1998) introduces the concept of organizational clockspeed by analogy. Organizations in fast clockspeed industries are compared to fruit flies and organizations in slower clockspeed industries to less rapidly evolving species. "Clockspeeds are to business genetics what lifecycles are to human genetics" (p. 17). In Fine's view, organizations must evolve to meet new challenges or die, just as species evolve for survival, and companies in low clockspeed industries can benefit from studying companies in high clockspeed industries (the fruit flies of industry).

An industry's clockspeed is increased with increases in technological innovation and competitive intensity. Of considerable importance are the advances of 
semiconductor and fiber optics technologies since these advances have contributed to increases in clockspeed of almost all industries (p. 26). With our global economy, every innovation in information and communication industries affects all industries worldwide simultaneously.

Two "laws of supply chain dynamics" are identified by Fine (1998):

- Volatility amplification, where in the supply chain “...volatility of demand and inventories in the supply chain tend to be amplified as one looks farther 'upstream' - that is away from the end user" (p. 89). This effect is described by Forrester (1958), demonstrated in the beer game by Sterman (1989), and is sometimes called the bullwhip effect.

- Clockspeed amplification, where clockspeeds tend to be amplified as one looks farther downstream toward the final customer (p. 97).

These two laws of supply chain dynamics are important due to the hierarchical and interdependent systems structure of the supply chain. Each hierarchical level of the supply chain adds more amplification in both volatility and clockspeed (p. 101). 
Guimaraes, Cook and Natarajan (2002) study the relationship of industry clockspeed to supplier network performance and the use of information technology, finding that increased information technology effectiveness improves supplier network performance in high clockspeed industries, but high clockspeed has a detrimental effect on supplier network performance where deeper relationships with few suppliers are used. Organizations in high clockspeed industries may need to develop "shallow" relationships with a relatively large number of suppliers.

Carillo (2005) identifies several characteristics of fast clockpeed industries (p. 139):

- High or increasing marginal net revenue earned per unit

- Low discount rates

- Low development cost structures

- High and / or growing total market of potential buers

- Technological and organizational barriers

Characteristics of low clockspeed industries include:

- Low initial marginal net revenue earned per unit

- High development cost structures

- Low and /or shrinking total market of potential buyers 
Three "submetrics" of clockspeed are considered by Fine (1998): process clockspeed, product clockspeed, and organizational clockspeed (p. 17). These submetrics are considered relative to a selection of sample industries as shown on Table 1: 
TABLE 1

SAMPLE INDUSTRY CLOCKSPEEDS

\begin{tabular}{|c|c|c|c|}
\hline \multicolumn{4}{|c|}{ Measuring Clockspeed - Sample Industries } \\
\hline Industry & $\begin{array}{c}\text { Product } \\
\text { Technology } \\
\text { Clockspeed }\end{array}$ & $\begin{array}{c}\text { Organization } \\
\text { Clockspeed }\end{array}$ & $\begin{array}{c}\text { Process } \\
\text { Technology } \\
\text { Clockspeed }\end{array}$ \\
\hline \multicolumn{4}{|c|}{ FAST-CLOCKSPEED INDUSTRIES } \\
\hline Personal computers & $<6$ months & 2-4 years & 2-4 years \\
\hline $\begin{array}{l}\text { Computer-aided } \\
\text { software engineering }\end{array}$ & 6 months & 2-4 years & 2-4 years \\
\hline Toys and games & $<1$ year & 5-15 years & $5-15$ years \\
\hline Athletic footwear & $<1$ year & 5-15 years & 5-15 years \\
\hline Semiconductors & $1-2$ years & 2-3 years & 3-10 years \\
\hline Cosmetics & $2-3$ years & $5-10$ years & $10-20$ years \\
\hline \multicolumn{4}{|c|}{ MEDIUM-CLOCKSPEED INDUSTRIES } \\
\hline Bicycles & 4-6 years & $10-15$ years & 20-25 years \\
\hline Automobiles & 4-6 years & 4-6 years & $10-15$ years \\
\hline $\begin{array}{l}\text { Computer operating } \\
\text { systems }\end{array}$ & $5-10$ years & $5-10$ years & $5-10$ years \\
\hline Agriculture & 3-8 years & $5-10$ years & $8-10$ years \\
\hline Fast food & 3-8 years & $25-50$ years & $5-25$ years \\
\hline Beer brewing & 4-6 years & 400 years & 2-3 years \\
\hline Airlines & $5-7$ years & $\begin{array}{l}25 \text { years } \\
\text { (hardware) } \\
2-3 \text { years } \\
\text { (software) }\end{array}$ & $<5$ years \\
\hline Machine tools & $6-10$ years & $6-10$ years & $10-15$ years \\
\hline Pharmaceuticals & 7-15 years & $10-20$ years & $5-10$ years \\
\hline \multicolumn{4}{|c|}{ SLOW-CLOCKSPEED INDUSTRIES } \\
\hline Aircraft (commercial) & $10-20$ years & $5-30$ years & 20-30 years \\
\hline Tobacco & $1-2$ years & 20-30 years & 20-30 years \\
\hline Steel & 20-40 years & $10-20$ years & $50-100$ years \\
\hline Aircraft (military) & 20-30 years & $5-30$ years & $2-3$ years \\
\hline Shipbuilding & 25-35 years & $5-30$ years & $10-30$ years \\
\hline Petrochemicals & $10-20$ years & 20-40 years & 20-40 years \\
\hline Paper & $10-20$ years & 20-40 years & 20-40 years \\
\hline Electricity & 100 years & $25-50$ years & $50-75$ years \\
\hline Diamond mining & Centuries & 20-30 years & $50-100$ years \\
\hline (Fine, 1998, p. 239) & & & \\
\hline
\end{tabular}


Mendelson and Pillai (1999) look at industry clockspeed relative to measurements and operational implications and find that industry clockspeed is related to internal operations and organizational clockspeed, “...the frequency with which a firm redesigns its products, the duration of its development projects, the speed at which its manufacturing operations are stabilized, and the likelihood of organizational restructuring, all correlate very strongly with the clockspeed of its industry environment" (p. 2). In Mendelson and Pillai's study, three components are used to measure clockspeed:

- The fraction of total revenue derived from new products (i.e., introduced within the preceding twelve months) - an indicator of product innovation;

- The total duration of the product life cycle (i.e., product life); and

- The rate of decline in the prices of input materials (p. 3).

Carillo (2005) looks at new product development clockspeed (using average rate of new product introduction for a particular industry). Similar to Mendelson and Pallai's findings, Carillo notes that by focusing "...on the new product development dimension of clockspeed...[they] also capture the impact of process and organization dimensions on the firm's ability to bring new products to market effectively" (p. 126), though more work is needed to develop “....appropriate measures and managerial guidance for the process and organization dimensions of clockspeed" (p. 139). 


\section{Systems Definitions and Concepts}

A system as defined by Lendaris (1986) is: “a) a unit with certain attributes perceived relative to its (external) environment, and, b) that unit has the quality that it internally contains subunits, and these subunits operate together to manifest the perceived attributes of the unit" (p. 604). A system is a subsystem of supra (meta) systems, and is also a supra (meta) system of other systems (subsystems). In other words, the Level A system is the Level B subunit of other systems, and the Level B subunit is the Level A system of other Level B subsystems. The boundaries of a system are established by definition (selecting the "perceived attributes" and subunits which operate together to manifest the emergent properties of those perceived attributes). In the case of economics, Simon (1981) describes this hierarchical level relative to man, markets and economy:

Among all the social sciences, economics exhibits in purest form the artificial component in human behavior and does so at three or more levels" the level of the individual actor (economic man or business firm), the level of markets, and the level of an entire economy. At all these levels the outer environment is defined by available technologies and by the behavior of other economic actors, other markets, or other economies. The inner environment is defined by the system's goals and by its capabilities for rational, adaptive behavior" (p. 31).

Another basic characteristic of systems is circularity as opposed to linearity.

Systems are composed of loops and delays. Senge (1990) deals with this concept in great detail with his description of basic archetypes noting the effects of feedback. An example of a simple feedback loop he uses is the process of filling a glass with water. One starts with an actual level of water that is less than the desired level of water in the 
glass. Since the actual level (reality) is less than the desired level, the action is to turn on the faucet. As the glass fills up, the reality level more and more closely approximates the desired level, followed by the action of turning the faucet thus reducing the flow. This is a repeating looped cycle (process) which repeats until the actual level (reality) equals the desired level and the faucet is turned off. A similar system, with the addition of a delay, is the process of adjusting the temperature of water for a shower. The longer the delay between turning the faucet and the adjusted temperature (feedback), the more difficult it is to achieve desired temperature (more iterations are required). Taking the delay into account and waiting until feedback is received before taking modifying action (turning the faucet) can speed up this process by reducing the number of iterations required.

Based on an understanding of feedback loops and delays, a number of archetypes with varying degrees of complexity have been identified by Senge (1990) that explain common problems that are faced on a regular basis. The archetypes offer leverage because they restructure the information to show the structural simplicity underlying the complexity of the problem. Non-leverage actions are frequently used (symptomatic solutions), but they do not address the fundamental problem, resulting in recurrence of the problem and the need to apply the symptomatic solution again. When the fundamental problem and solution are identified by the archetype, the problem can be addressed in a way that eliminates the recurring pattern.

All organizations are subsystems of a larger system, the supply chain, which Fine (1998) proposes to view as “...consisting of three strands: a chain of organizations, a 
chain of technologies, and a chain of capabilities" (p. 13), and the organization's core capabilities must therefore be assessed in context of capability chains. In an organizational setting, the organization is the Level A system and individuals (employees/agents) are the Level B units of the system. According to Senge (1990), leverage for functionality of the organizational system is available through recognition of growth of the individual units and their interactions. Growth of the individual units is achieved by pursuit of Personal Mastery (PM). PM requires an awareness of deeply held beliefs (mental models) that may limit the ability to create what is desired. Reflection skills help increase awareness of mental models and recognition of leaps of abstraction (a core element of misunderstanding). To reflect and recognize leaps of abstraction beliefs about the way the world works, about the nature of business, about people in general and about specific individuals must be questioned. Once generalizations are recognized, determination of what data the generalization is based on and a decision of whether the generalization may be inaccurate or misleading must be made. PM also requires a commitment to principles and values, utilization of the rational mind and the intuitive mind, an awareness of oneself as an individual, an awareness of ones place within the organization, and an awareness of ones place within the world at large. Finally, PM requires creative tension (the gap between desire and reality) to promote progress toward goals or visions which are driven by purpose, thereby maintaining cycles of growth.

Leverage in the organization through interactions of the units is found in developing shared vision (purpose) and alignment of personal visions with organizational visions (team learning) which maximizes the utilization of energy. The concept of 
archetypes noted above apply to the relationships of Level B units within the organizational Level A system, and can be used to help promote the shared vision and alignment that are desired.

Given knowledge of the impact of mental models on the individual and the organization, it is advisable to view problems from multiple perspectives when reviewing problem resolution from the past or attempting to anticipate and prevent problems in the future. Multiple perspectives are identified and defined by Linstone (1999) as follows:

- Technological Perspective: The United States as a culture is the most strongly technologically oriented culture in the world. This orientation is pervasive to the point that we rate beauty, quality of life and other aesthetic qualities in quantitative terms. The advent of the computer as a tool for analysis, and its utility in the technological orientation, has strengthened the technological perspective as the decision-making perspective of choice.

- Organizational Perspective: The organizational perspective reflects the culture and myths of the organization and helps bind the organization into a distinct entity in the eyes of the members. This perspective is of crucial importance for the perception of reality and is intimately bound to the need for security (belonging). The organizational perspective is related to power, and as such is an important perspective to utilize in attempting to affect change in the social environment. History is replete with examples of this perspective being used to promote destruction. Where is the 
real leverage? How can conflicts among units be turned to constructive use? It has been documented that group opinion can modify or distort individual judgment. This can be used to affect desired change, but should also serve as a warning to beware.

- Personal Perspective: Communication of complex problems and issues may be made more effective by means of the personal perspective. Six million murders in the holocaust vs. Diary of Anne Frank impacts perception at a level not possible by the analytic mind. One "real" death versus statistics highlights the personal perspective grasp of reality not possible with statistics alone. In the organizational environment, effective organizations are those that have found successful ways of making the selfinterest of the members work constructively and in unison to support the goals of the organization.

In addition to utilizing multiple perspectives, a variety of inquiring systems (IS) have been identified by Mitroff and Turoff (1973) that help understand the environment and the nature of the problem in question:

- Leibnizian IS: The epitome of formal, symbolic systems. Strives to reduce problems to a formal mathematical or symbolic representation. Most appropriate for working on clearly definable, well-structured problems for which there exists an analytic formulation and solution. Theoretically, deductively derived models. Emphasizes theory to the detriment of data. 
- Lockean IS: The epitome of experimental, consensual systems. Emphasizes empirical, inductive representations of problems. Best suited for working on wellstructured problem situations for which there exists a strong consensual position on the nature of the problem situation. Empirically, inductively derived models emphasize data to the detriment of theory.

- Kantian IS: The epitome of multimodal synthetic systems. On any problem will build at least two alternate representations or models (if complementary is Kantian IS, if antithetical is Hegelian IS). Places emphasis on alternate models in dealing with problems to get as many perspectives on the nature of the problem as possible. Best suited to problems which are inherently ill-structured and inherently difficult to formulate in pure Leibnizian or Lockean terms.

- Hegelian IS: The epitome of conflicting, synthetic systems. Emphasizes creation of at least two, completely antithetical, representations. May start with two strongly opposing Leibnizian models of a problem. Premise is that data are not information, information is that which results from the interpretation of data and out of a dialectic confrontation between opposing interpretations, the underlying assumptions of the opposing Leibnizian models will be brought to the surface for conscious examination.

- Singerian IS: The epitome of synthetic multimodal, interdisciplinary systems (metaIS). Theoretical unification of previous paradigms includes all the previous IS as sub models in the design. Offers a theory about how to manage the application of the 
other types of IS. Speaks almost exclusively in the language of commands.

Attempts to draw hidden commands out of every system so that the analyst is better able to choose the commands he wishes to postulate. Shows how it is possible to incorporate ethics into the design of any given system. If a command underlies every system, it can be shown that behind every system is a set of ethical presuppositions.

Systems have been defined and various aspects of systems have been identified. A Systems Methodology also exists and is articulated by Hall (1989). The function of systems methodology is to create efficient open systems or entities which satisfy one or more goals. The methodology focuses on systems as wholes, and on their parts taken separately only as they relate to properties of the whole. Systems Methodology is distinct from the formal sciences and the factual sciences due to the use of value truths in addition to formal truths (which do not depend on meanings, but only relationships), and factual truths (which depend on the truth of its components). Value truths are subjective and nontransferable, and are used to define wanted systems. Value system design is found in every phase of systems methodology, and it employs a complete subcycle of metasystem synthesis, metasystem analysis and metasystem optimization. The value system design concludes with specific tentative metasystem, that provides the logical basis for the concrete system design.

If Systems Methodology is considered to be the Level A system, the Level B elements in the logic of systems methodology are as follows: 
- Problem definition, including environmental forecasting and impact assessment, in principle must be done over the entire system life cycle. Essential activities include isolating, quantifying, and clarifying the need which creates the problem, and describing the set of environmental factors that define the system and its environment. Describes the operational situation, user requirements, legal considerations, and possible system inputs and outputs.

- Value system design, or metasystem design, or normative scenario production, means to select the set of objectives and goals that will: 1) guide the search for alternatives, 2) imply the types of analyses required of the alternatives, and 3) provide the multidimensional decision criterion for selecting the most appropriate ("optimum") system.

- Systems synthesis, or collecting, searching for, or inventing a set of ideas, alternatives, or options. Each alternative must be worked out in enough detail to permit its subsequent evaluation with respect to the objectives, and to permit an application of the multidimensional decision criterion to decide its relative merits for proceeding into the next phase.

- Systems analysis means determining the relevant consequences in terms of the value system. These deductions may relate to quality, market, reliability, cost, effectiveness, quality of life, freedom, privacy, etc. 
- "Optimization" of the alternatives, or proportioning the system variables to meet the objectives, entails interaction of the first four steps, often by using a model for selected system attributes which may be useful in the proportioning.

- Decision making involves evaluating the consequences of the alternatives developed in systems analysis relative to the objectives, and incorporating these evaluations into the decision criterion so that all alternatives can be compared relative to the criterion, to the end that one or more alternatives can be selected for advancing to the next phase.

- Planning for action to implement the next phase includes communicating the results of the process to this point, scheduling subsequent efforts, allocating resources to carry out the work, assigning priorities for subsequent action, setting up a management control system consisting of performance criteria and feedback methods. If we were not modeling a multi-phase system, this step for starting and controlling action would be final.

Complexity is another systems concept that warrants review since as noted by Pagels (1988) “...the mind can hold at most $7 \pm 2$ distinct items before its attention” (p. 41). Pagels goes on to look at complexity from several different perspectives:

- Algorithmic complexity is the length of the minimal program required to compute something. This concept was described by Alan Turing who “...distinguished 
between 'computable' and 'noncomputable' numbers..." using the example of 3 divided by 7 (pp. 55-56). The complexity of the resultant number is high if one were to try to define each sequential digit, as in defining a number derived by successive random roles of a die $[0.42857142 \ldots]$, but the program to derive the number $3 / 7$ is quite short. Based on the concept of algorithmic complexity the appearance of complexity may, at its root, not be complex. "For 'computable' numbers, even if they are infinitely long, it is possible to write a relatively short program that will calculate them" (p. 56). Algorithmic complexity is “... a definition of randomness..." (p. 64).

- Computational complexity is a measure of the time it takes to solve a problem. Computational complexity exists in two general categories, those “...that require a geometrical (exponential) amount of computing time and those that require only an arithmetic (power law) amount of computer time" (p. 62). One classic problem of the geometrical type is the traveling salesman problem where the salesman has a certain number of cities to visit while traveling the shortest distance upon return to the start point. Problems of the geometrical type are suggested to require in their extreme " $\ldots$ millions of lifetimes of the universe to be solved... "(p. 62). "Algorithmic complexity is in a sense a measure of complexity in space (the length of the minimal algorithm); computational complexity is a measure of complexity in time (the time it takes to solve a problem) as well as in space" (p. 61). 
- Information-based complexity is based on the clarity of information. Is the problem completely specified and known or is the available information partial or contaminated. Information-based complexity is relevant to real world problems such as performance measures. Lack of clarity of information is referred to as "noisiness" of information by Feltham and Xie (1994) and the quality of information as "informativeness" by Ramachandran (2004).

- Physical complexity is based on the diversity of a system in a hierarchical manner. This hierarchy can correspond to the structural layout of the system or, to clustering the parts by the strength of interactions. "In particular, if the most strongly interactive components are grouped together and this procedure is repeated with the resulting clusters, one produces a tree [like an organization chart] reflecting the hierarchy of the system. Once such a hierarchy has been defined it is possible to assign a measure to its complexity, taking into account diversity in interactions among the components" (p. 65).

- Logical depth is defined by how hard it is to put something together starting from elementary pieces, “....measured by how long it takes to simulate the full development of that object beginning with the elementary algorithm and taking no short cuts" (p. 67). 
According to Simon (1981) "Economics ...illustrate[s] how outer and inner environment[s] interact, and in particular, how an intelligent system's adjustment to its outer environment (its substantive rationality) is conditioned by its ability to discover appropriate adaptive behavior (its procedural rationality)" (p. 31). Rationality is the attempt to optimize the allocation of scarce resources. Operations research is an applied science that contributes to achievement of procedural rationality by providing “...algorithms for handling difficult multivariate decision problems, sometimes involving uncertainty" (p. 34). Algorithms utilized in operations research include linear programming, queuing theory, linear decision rules for inventory control and production smoothing. Modeling the real world introduces complexity into the business firm's outer environment subsequently that increases the complexity of the inner environment. For the inner environment, constraints on adaptation include uncertainty about the outer environment and limits on the calculation capabilities available for solving the optimization problems. "The normative theory of the firm becomes a theory of estimation under uncertainty and a theory of computation—decidedly nontrivial theories[,] as the obscurities and complications of information and computation increase" (p. 34). This increase in complexity gives rise to "satisficing” decision. “... the decision that is optimal in the simplified model will seldom be optimal in the real world. The decision maker has a choice between optimal decisions for an imaginary simplified world or decisions that are "good enough," that satisfice, for a world approximating the complex real one more closely" (p. 35). 
Normative economics has shown that exact solutions to the larger optimization problems of the real world are simply not within reach or sight. In the face of this complexity the real-world business firm turns to procedures that find good enough answers to questions whose best answers are unknowable. Thus normative microeconomics, by showing real-world optimization to be impossible, demonstrates that economic man is in fact a satisficer, a person who accepts "good enough" alternatives, not because he prefers less to more but because he has no choice" (Simon, 1981, p. 36).

\section{Conclusions}

Performance measures have a long history of use had have been studied from a variety of different perspectives. To summarize:

- Not much evidence has been found to determine under which conditions financial and nonfinancial measures perform best (Hemmer, 1986) $\left(\mathrm{H}_{2-3}\right)$.

- Synergistic relationships between different measures are assumed to exist (Capon, et al., 1990; Chenhall \& Langfield-Smith, 1998) $\left(\mathrm{H}_{1-4}\right)$.

- Performance measurement systems are considered to be insufficient and do not support top managements’ business objectives (Ittern \& Larker, 1998) $\left(\mathrm{H}_{1-4}\right)$.

- Due to contingencies (including clockspeed $-\mathrm{H}_{3}$ ), the complexity of (and interaction with) the larger economic metasystem, the complexity of the organization itself, and trade-offs and noisiness (informativeness) of 
performance measures, managers rely on satisficing (Simon, 1981) rather than truly optimal decisions.

- There is a tendency to rely on the numbers generated by performance measures and not on the processes behind the numbers $\left(\mathrm{H}_{1}\right)$ (Johnson and Kaplan, 1987; Haponova, et al., 2006), highlighting the need to view performance measurement systems from a systems perspective [“...economic, social, behavioral, and managerial within an overall organizational context” (p. 381). (Otley, 1999)].

The next Chapter develops the theoretical basis for the questions and hypotheses under consideration using a systems perspective. 


\section{CHAPTER III \\ DESCRIPTION OF THE PROBLEM}

\section{Questions and Hypotheses}

Can one quantify the value of "managing process" with a systems perspective? As complex dynamic systems, organizations are compared to living organisms (Fine, 1998; Johnson \& Broms, 2000), a perspective that may be useful. Literature notes the value of nonfinancial measures (Chow \& Van der Stede, 2006), and even a preference for managing by nonfinancial measures because financial measures are after-the-fact (Banker, Potter \& Srinivasan, 2000; Lee, Kwak \& Han, 1995; Nagar \& Rajan, 2001), but using these measures does not fully address the issue. True, managing by nonfinancial measures gets one "closer" to the process, but this does not get to the heart of the process structure because it does not "quantify" the process structure or the "value added" components of the structure. Are there fundamental process related measures? Many companies identify Key Performance Indicators (KPI), but do these indicators truly represent "Key" processes driving profitability and success? The Balanced Scorecard proposed by Kaplan and Norton (1996) address the comprehensive nature of performance measurement requirements by including Financial, Customer, Internal Business Process, and Learning and Growth metrics. 
Perhaps a different view of business process is needed. As Collins and Porras (1997) state “...we're asking you to see the success of visionary companies—at least in part—as coming from underlying processes and fundamental dynamics embedded in the organization...“( (p. 41). A perspective of business process types that may address this issue is offered by Keen (1997). Keen's perspective includes four fundamental process types; 1) Identity, 2) Priority, 3) Background, and 4) Mandated. These four process types, defined as "process salience," are as follows:

- An identity process is one that defines the company for itself, its customers, and its investors. It differentiates a firm from its competitors and is at the heart of the firm's success (p. 25). For FedEx the identity is rapid, reliable delivery of packages.

- Priority processes are the engine of corporate effectiveness. They strongly influence how well identity processes are carried out and how a firm stands relative to its competition...Priority processes tend to be invisible to the customer...but when they fail the problems are visible and immediate.... In the case of FedEx, a package delivery company, airplane scheduling and maintenance would be priority processes. For McDonald's, food supply management is a priority process. Customers think of McDonald's as a family restaurant, not a food distributor. They will not give food-supply management a thought unless the process breaks down, and they discover they can't get fries with their Big Macs (p. 26).

- Background processes are a necessary support to daily operations. Many administrative and overhead functions are background processes. For most companies, office management, document management, accounting, and many other common administrative processes are background. They are often the core of daily operations, but it is a mistake to allow their visibility to make them the main target for management attention and capital investment, because improving them rarely generates much EVA [Economic Value Added] (p. 27).

- Mandated processes are those a company carries out only because it is legally required to do so. Regulatory reporting and filing tax returns are obvious examples (p. 27). 
A fifth process type is also noted, folklore processes, which exist only because they were implemented in a previous time period but offer no functionality in the current environment. According to Keen, resources in general should be allocated to processes that will generate positive EVA (Economic Value Added) as defined by Stewart (1991):

Economic value added (EVA) is the one measure that properly accounts for all the complex trade-offs involved in creating value. It is computed by taking the spread between the rate of return on capital $r$ and the cost of capital $c^{*}$ and then multiplying by the economic book value of the capital committed to the business:

$\mathrm{EVA}=\left(r-c^{*}\right) \mathrm{x}$ capital

$\mathrm{EVA}=($ rate of return - cost of capital) $\mathrm{x}$ capital (p. 136).

Stewart (1991) also notes that:

...EVA increases when:

1. The rate of return earned on the existing base of capital improves; that is, more operating profits are generated without tying up any more funds in the business.

2. Additional capital is invested in projects that return more than the cost of obtaining the new capital.

3. Capital is liquidated from, or further investment is curtailed in, subsequent operations where inadequate returns are being earned. (p. 137)

Keen (1997) assesses processes using a "Salience Worth Matrix", where

Salience is the above noted process type and Worth is defined as an asset if the process generates positive EVA and as a liability if the process generates negative EVA.

Assuming that managers allocate more resources in the form of controls and performance 
measurement to the processes they deem more important than to those they deem less important, and also assuming that managerial decisions are based on these processes being monitored, it can be assumed that process measurement targeting correlate to management intervention and impact firm value accordingly, leading to the first hypothesis of the dissertation:

$\mathrm{H}_{1-0}$ : Salience of business processes identified for measurement do not correlate to firm value as tested by Kruskal-Wallis one-way ANOVA.

$\mathrm{H}_{1}$ : Salience of business processes identified for measurement correlate to firm value as tested by Kruskal-Wallis one-way ANOVA.

$\mathrm{H}_{1 \mathrm{a}-0}$ : Identity business processes using the means results from $\mathrm{H}_{1}$ do not have a higher positive correlation to firm value than Priority business processes as tested by the Chi-Square Contingency Test of Association.

$\mathrm{H}_{1 \mathrm{a}}$ : Identity business processes using the means results from $\mathrm{H}_{1}$ have a higher positive correlation to firm value than Priority business processes as tested by the Chi-Square Contingency Test of Association.

$\mathrm{H}_{1 \mathrm{~b}-0}$ : Priority business processes using the means results from $\mathrm{H}_{1}$ do not have a higher positive correlation to firm value than Background business processes as tested by the Chi-Square Contingency Test of Association. 
$\mathrm{H}_{1 \mathrm{~b}}$ : Priority business processes using the means results from $\mathrm{H}_{1}$ have a higher positive correlation to firm value than Background business processes as tested by the Chi-Square Contingency Test of Association.

Lee et al. (1995) note that one problem with accounting (financial) performance measures is that they are "...lagging indicators, [based on] historical statements of financial performance...the result of management performance, not the cause of it" (p. 344). In Figure 2 it would mean that Delay $2>$ Delay 1.

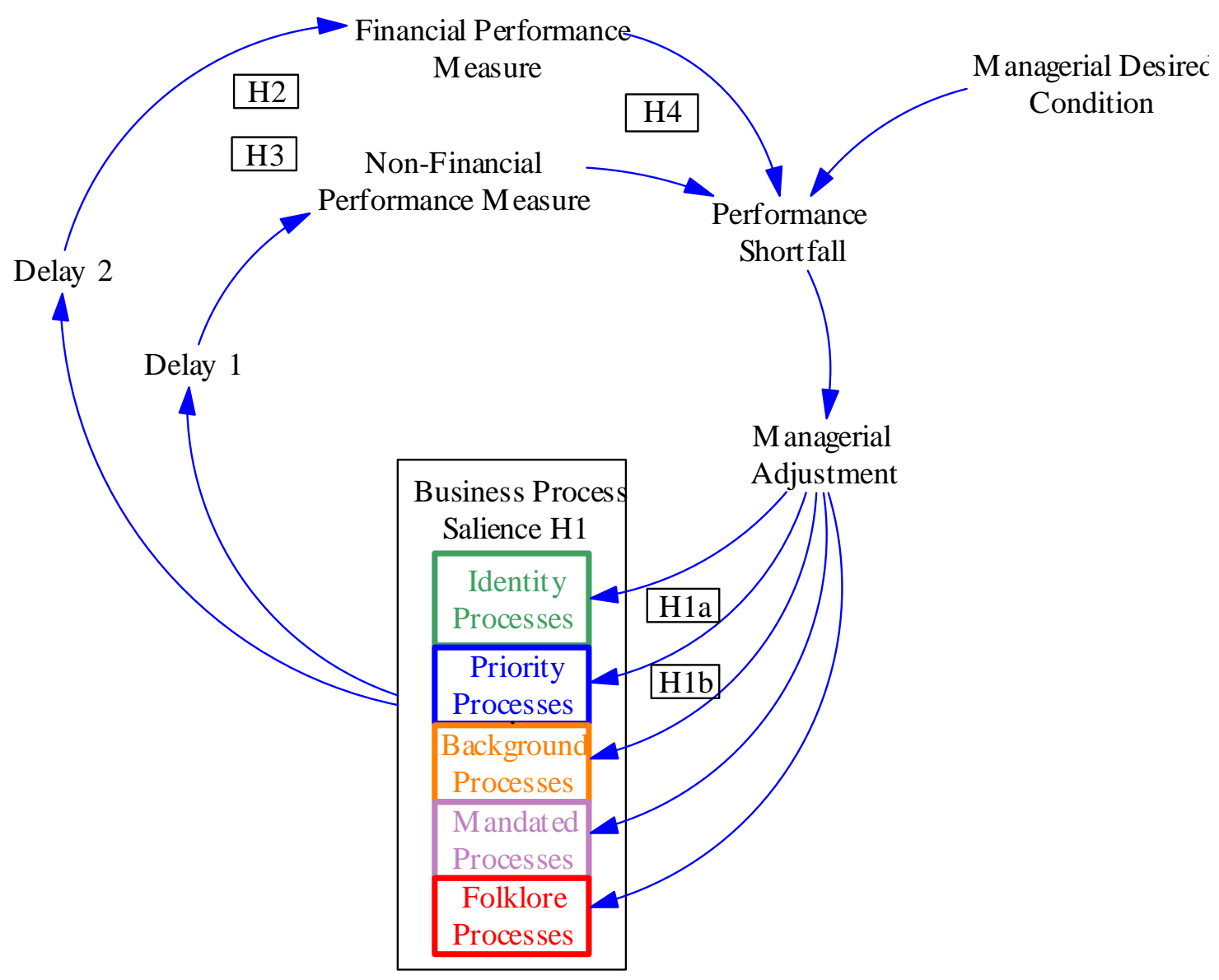

Figure 2: Business Processes and Performance Measure Feedback 
In a study by Nagar and Rajan (2001) financial measures are shown to be a leading indicator with correlation to sales $2-3$ quarters in the future as compared to nonfinancial measures correlation to sales the following quarter (p. 496). They note that this financial "leading indicator" is not typical since financial measures are considered lagging indicators rather than leading indicators. Though the financial measure (external failure cost) in this case is a leading indicator, the law of transitivity applies and correlation between the nonfinancial measure (defect rates) and the 2-3 quarter lagged financial measured effect would exist since the effect measured by the nonfinancial measure is the root cause of the financially measured effect. Following this logic, the nonfinancial measure would correlate to both sales one quarter lagged, and to sales several quarters lagged, and be the fundamental measurement Figure 3). 


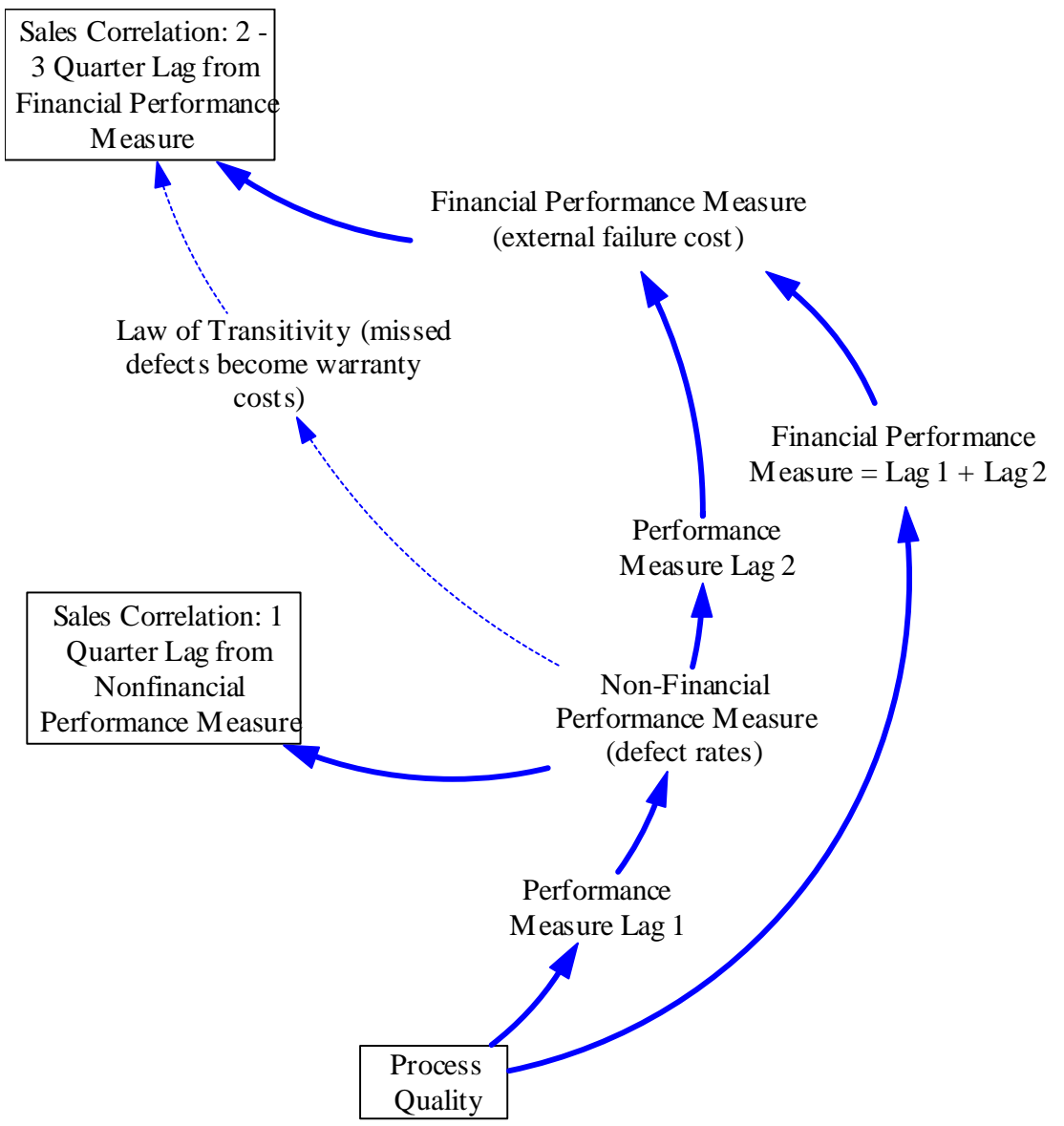

Figure 3: Financial Measures vs. Nonfinancial Measures Lagged Effect

Nagar and Rajan (2001), in reference to another study by Luft and Shields note that in an experimental setting individuals can assess the future financial impact of quality more accurately using nonfinancial quality measures than using financial quality measures (p. 496). Banker, Potter and Srinivasan (2000) state that “...there have been very few studies on the relation[ship] between nonfinancial measures and financial performance, and the empirical evidence provided by these studies has been mixed..." (p. 66). An assumption can be made that the reduction of lagged effect of nonfinancial 
performance measures will result in earlier intervention and problem resolution, thereby positively impacting profitability and lead to the next hypothesis:

$\mathrm{H}_{2-0}$ : Non Nonfinancial performance measures are not more correlated to firm value than financial measures as tested by Kruskal-Wallis one-way ANOVA.

$\mathrm{H}_{2}$ : Nonfinancial performance measures are more correlated to firm value than financial measures as tested by Kruskal-Wallis one-way ANOVA.

Given that nonfinancial performance measures result in shorter lag periods of feedback than financial performance measures, the relative importance of the lag time will be heightened in higher clock-speed industries as defined by Fine (1998); with higher clock-speed industries being more susceptible to dynamic archetypes associated with delayed feedback (Senge,1990; Sterman, 2000), and thus leads to a third hypothesis:

$\mathrm{H}_{3-0}$ : The relative importance of nonfinancial performance measures compared to financial measures using the results from $\mathrm{H}_{2}$ is not greater in high clock-speed industries than in low clock-speed industries as tested by Cramer's Phi.

$\mathrm{H}_{3}$ : The relative importance of nonfinancial performance measures compared to financial measures using the results from $\mathrm{H}_{2}$ is greater in high clock-speed industries than in low clock-speed industries as tested by Cramer's Phi. 
In an organizational setting, performance measures are the feedback mechanism used to pass information back to the controller (manager). According to Rubinstein (1975):

Feedback control is a key to survival; it has endowed all living organisms with the system characteristics most productive for survival. The main feature in control with feedback is the flexibility which can be exercised by the controller to vary the input, leading to a change in output. The species [organization] with a high degree of specialization were [is] less likely to adapt to disturbances from the environment and, therefore, less likely to survive (p. 413).

This study is interested in information theory and dynamic system delays in organizational feedback systems since people perform poorly with delays in dynamic system feedback structures (Diehl \& Sterman, 1995; Sterman, 2000, p. 26). Differing types of information (type of performance measure) have differing lag periods. The closer the information ties to the business process, the less the delay and the better people are able to work with the information. In Johnson and Brohms (2000) work, two examples are given of nonfinancial performance measures that are identified as largely contributing to the success of the organization. The first is the Takt time (rate at which vehicles are released to customers) used at a Toyota manufacturing facility to establish and maintain a continuous flow of product and information throughout the system (p. 88). This takt time is used to define the rate that each work-station completes its part of the process. Work orders are released from within the process in such a way that future components meet product process as they are needed. "Throughout the entire plant, the flow of information from both external and internal customers initiates and directs how 
material flows from worker to worker" (p. 83). The information flow is portrayed as "...a web of unbroken, interconnected relationships" (p. 38), with the further clarification that "The point to recognize... is that in a balanced continuous flow...the work is the information and all the information needed to direct operations is in the work"(p. 31). The second nonfinancial performance measure noted by Johnson and Broms (2000) is the density matrix of Scania, where the modularity benefit based on the engineered part is defined before the part is ever introduced into production, with no resultant delay in feedback relative to production. This measurement structure is engineered into the process. Bourne, Kennerley, and Franco-Santos (2005) study the use of non-standard measurements in high-performing business units as compared to medium-performing business units. Their research finds that high-performing branch managers use "simple mental models" to manage their business units on a daily basis. They often use their own metrics instead of the formal Balanced Scorecard that the organization uses (p. 382) and ignore inappropriate targets (p. 384). They proactively reduce the typical lag period found in average performing units that wait for weekly reports.

Can an analogy of Organization to Organism offer intuitive value in developing mental models that are simple and effective? Organisms evolve and develop effective means of adapting for survival. What feedback mechanisms in organisms can be effectively modeled in context of an organization to provide value? The Takt rate of Toyota is compared to the pulse rate of an organism, the modular structure of the Scania density index is compared to the cellular structure of organisms. Fine (1998) compares the clock-speed of industries to the life cycle of organisms, and the cyclic nature of 
"integrated product vertical industry" / "modular product horizontal industry" to the double helix DNA [this cyclic pattern is an oscillating system]. Brown, Hitchcock and Willard (1994) identify the dynamic aspect of feedback measures required to succeed. They find "To succeed in turbulent times, we need more and more frequent feedback, not less" (p. 107). The ability to change quickly in a world of change is essential for survival and the organism has a need to monitor feedback from all parts of the system for health and longevity. In living organisms, the comprehensive network is exhibited by the autonomic nervous system. In this study, this "autonomic" comprehensive feedback network is considered analogous to the Balanced Scorecard concept of maintaining total organization health by monitoring not only financial, but also customer, internal business process, and learning and growth metrics, giving us the final hypothesis of this study:

$\mathrm{H}_{4-0}$ : The number of categories of Balanced Scorecard metrics used does not correlate to firm value as tested by Kruskal-Wallis one-way ANOVA.

$\mathrm{H}_{4}$ : The number of categories of Balanced Scorecard metrics used correlate to firm value as tested by Kruskal-Wallis one-way ANOVA.

Banker et al. (2000) state that "Recent studies report an increasing use of nonfinancial measures such as product quality, customer satisfaction, and market share in performance measurement and compensation systems (p. 65)", but they also note that there is not much empirical evidence regarding the relationship between nonfinancial 
measures and financial performance. Studies investigating the correlation of performance to independent variables, or combinations of those variables have failed to investigate the relationship of the measures to the process structure. The salience of processes under consideration as defined by Keen (1997), in conjunction with the Kaplan and Norton (1996) multi-attribute strategy, can be used as a proxy to "quantify" the process structure, the "value added" components of the structure, and the value of managing based on that structure. Identity and Priority processes are most closely linked to generation of positive EVA, and therefore to contribution to the survival of the organization. This relates to living systems which develop control feedback systems for the organism's survival (identity and infrastructure). The objective of this research project is to advance the field of study by finding synergies in the dynamic interactions of financial/nonfinancial, objective/subjective, internal business, customer, learning and growth, identity, priority, background and mandated processes.

Van der Stede et al. (2006) studies the value of financial, nonfinancial objective, nonfinancial subjective, and diversity of these alternative measurements relative to quality manufacturing. Kaplan and Norton (1996) introduced the balanced scorecard multi-attribute concept studied by Osama (2006) with Financial, Internal Business, Learning and Growth, and Customer metrics, and finally, Keen offers a perspective based on Identity, Priority, Background and Mandated processes. This objective of this research is to find synergies in the integrated combination of these perspectives as itemized in Table 2: 
TABLE 2

PERFORMANCE MEASURES INTEGRATED PERSPECTIVE

\begin{tabular}{|c|c|}
\hline \multicolumn{2}{|c|}{ Performance Measures } \\
\hline System & Breakdown \\
\hline $\begin{array}{l}\text { Balanced Scorecard (BSC) } \\
\left(\mathrm{H}_{4}\right)\end{array}$ & $\begin{array}{l}\mathrm{F}=\text { Financial } \\
\mathrm{IB}=\text { Internal Business } \\
\mathrm{C}=\text { Customer }\end{array}$ \\
\hline Kaplan \& Norton (1996) & $\mathrm{LG}=$ Learning and Growth \\
\hline $\begin{array}{l}\text { Process Salience } \\
\left(\mathrm{H}_{1}\right) \\
\text { Keen }(1997)\end{array}$ & $\begin{array}{l}\text { ID = Identity } \\
\mathrm{P}=\text { Priority } \\
\mathrm{B}=\text { Background } \\
\mathrm{M}=\text { Mandated }\end{array}$ \\
\hline $\begin{array}{l}\text { Measurement Type } \\
\left(\mathrm{H}_{2-3}\right)\end{array}$ & $\begin{array}{l}\mathrm{F}=\text { Financial } \\
\mathrm{N}=\text { Nonfinancial } \\
\mathrm{S}=\text { Subjective }\end{array}$ \\
\hline Van der Stede et al. (2006) & $\mathrm{O}=$ Objective \\
\hline
\end{tabular}

Chapter IV now defines the methods and techniques used to test these hypotheses. 


\section{CHAPTER IV}

\section{METHODS AND TECHNIQUES}

This research will look for performance correlation (synergies) with select combinations of performance measures. The hypothesis is that more productive combinations will be those with Process Salience Identity (ID - $\mathrm{H}_{1 \mathrm{a}}$ ) and Priority (P $\left.\mathrm{H}_{1 \mathrm{~b}}\right)$, use of Nonfinancial $\left(\mathrm{H}_{2}, \mathrm{H}_{3}\right)$ measures combined with multiple Balanced Scorecard (BSC) type measurements $\left(\mathrm{H}_{4}\right)$.

Atkinson and Shaffir (1998) advise of the potential for bias in management accounting field research and the need to confirm four primary requirements: 1 ) Construct Validity, 2) Internal Validity, 3) External Validity, and 4) Reliability. These four primary requirements are addressed in the proposed research as follows:

- Construct Validity: “...asks whether we are measuring what we want to measure. The major threats to construct validity are those created by bias either through the process of observing itself, or bias introduced by the observation method" (Atkinson \& Shafir, 1998, p. 60). The potential for introduction of bias by the observation method or process of observing is mitigated by the Survey Consent Form (see Appendix D), which states the 
subject matter of the research, the mode of observation, and the background training taken into the study.

- Internal Validity: “...asks whether the researcher has taken steps to ensure that the evidence used to infer a casual $[$ sic $]$ relationship is complete. That is, can we avoid reporting a spurious correlation as causal" (Atkinson \& Shafir, 1998, p. 61). The theoretic basis of the proposed correlation is defined in Chapter 3: Description of the Problem. Results will be compared based on company size, department, and staff position to test for spurious correlation.

- External Validity: “....asks whether we identified clearly the population to which our results apply" (Atkinson \& Shafir, 1998, p. 61). The definition of the subject population and the means of selection are defined in Chapter 4; Methods and Techniques. Statistical significance is expected based on a relatively large sample size solicited by email to participate in a web based survey.

- Reliability: “...asks whether the research can be replicated with the same results" (Atkinson \& Shafir, 1998, p. 62). This research encompasses a large number of industries with results standardized by industry (NAICS Code) over the same time period (end date of financial comparison will be uniquely defined and identical for all subjects). The following methods 
will be used to test reliability of the survey instrument: 1) Pilot survey to test questionnaire, 2) Review demographics of non-respondents vs. respondents (size, performance, NAICS category, longevity) to test nonresponse bias, 3) perform Split-Half reliability test, and 4) Review consistency among organization level responses relative to department and staff position.

\section{Web Based Survey}

Surveys are a well-utilized tool for conducting research. In addition, the World Wide Web has been finding increasing utility in surveys (DePaolo, \& Sherwood, 2006; Gun, 2002; Roztocki, \& Schultz, 2003; Schmidt, 1997) and simulations (Goosen, Wolfe, \& Gold, 2007; Thavikulwat, 2007; Thavilulwat, \& Chang, 2007). An email solicitation will be sent to various companies to participate in the Web Survey, which is composed of five tiers as follows (See Questionnaire, Appendix E):

North American Industry Classification System (NAICS) to benchmark performance Company (coded to provide confidentiality)

Rank (1- Executive, 2- VP, 3- Front Line Supervisor, 4- Staff)

Department (Corporate, HR, Finance, Marketing, Production) Employee (anonymous) 
The determination of the number of financial and nonfinancial measures used are provided by the respondent with a set of alternative measures provided for the respondent to consider. The Balanced Scorecard criteria (Financial, Learning and Growth, Customer, Internal Process) are structured into the survey. The Keen metrics (Identity, Priority, Background, and Mandated) need to be defined by the survey respondent. According to Keen (1997):

Analyzing the salience of a firm's processes is an important task that requires considerable thought and insight, even under the guidance of the salience/worth matrix. As we have seen, one of several complexities to be considered is that different groups and individuals see the salience of the same process differently. So the question "Whose valuation counts most? Must be answered before a process's importance to the entire firm can be determined. (p. 54).

Keen (1997) suggests that "While asking themselves (and one another) which processes identify the firm, which are critically important, which provide necessary support, and so forth, they [managers] should also consider how business environment changes are likely to affect their processes" (p. 56). The questionnaire will, therefore, request the respondent to rank from $1-4$ their use of the four process types.

In this research study, survey responses will be solicited from a variety of industries and from various staff levels within the organization (see Appendix F, NAICS Company Listings for subset of companies to be solicited). Consistency among organization level responses will give some indication of internal validity of response, 
though variances based on respondent function are expected. Industry categories will be defined to benchmark performance level.

The dependent variable is stock price (\% change) which reflects the impact of measures on firm value (stock price). The use of stock price as the dependent variable is implicitly supported by Keen (1997) who states "These processes-the ones investors care about - are the major processes that a company can and must pay attention to. They constitute the firm's process investment portfolio" (p. 56).

In order to perform the analysis: 1) the dependent variable benchmark for the various North American Industry Classification System (NAICS) categories under consideration must be defined, and 2) each company performance relative to the NAICS dependent variable benchmark must then be determined for testing. This process relative to NAICS 31621 is described in detail now:

\section{NAICS Company Definition}

NAICS 31621, "US Exchanges Only" is downloaded from Mergent Online as follows in Table 3. 
TABLE 3

NAICS 31621 COMPANY LISTINGS

\begin{tabular}{|c|c|c|c|c|}
\hline \multicolumn{5}{|c|}{$\begin{array}{l}\text { http://www.mergentonline.com.proxy.lib.pdx.edu/compsearchresults.asp? } \\
\text { searchtype=compname\&searchtext=\&codetype=naic\&industrycode= } \\
31621 \& \text { Index=null\&country=null\&usonly=on\&bstype=codeandcountry }\end{array}$} \\
\hline \multirow{2}{*}{\multicolumn{5}{|c|}{$\begin{array}{l}\text { Prim NAICS } 31621 \\
\text { Footwear Manufacturing }\end{array}$}} \\
\hline & & & & \\
\hline \multicolumn{2}{|l|}{ US Exchanges Only } & \multicolumn{2}{|c|}{ Accessed: } & 9/12/2007 \\
\hline Company Name & \begin{tabular}{|l|l|} 
SIC & Exchange \\
\end{tabular} & Ticker & Active/In & Country \\
\hline Barry (R.G.) Corp. & \begin{tabular}{|l|l|l|}
3149 & ASE \\
\end{tabular} & DFZ & Active & United States \\
\hline Brown Shoe Co., Inc. & \begin{tabular}{|l|l|}
3144 & NYS \\
\end{tabular} & BWS & Active & United States \\
\hline Cole (Kenneth) Productions, Inc. & \begin{tabular}{|l|l|}
3143 & NYS \\
\end{tabular} & KCP & Active & United States \\
\hline Crocs Inc & \begin{tabular}{|l|l|}
3021 & NMS \\
\end{tabular} & CROX & Active & United States \\
\hline Deckers Outdoor Corp. & 3021 NMS & DECK & Active & United States \\
\hline Foot Locker, Inc. & \begin{tabular}{|l|l|}
5661 & NYS \\
\end{tabular} & $\mathrm{FL}$ & Active & United States \\
\hline Iconix Brand Group Inc & 3149 NMS & ICON & Active & United States \\
\hline K-Swiss, Inc & 3149 NMS & KSWS & Active & United States \\
\hline LaCrosse Footwear, Inc. & \begin{tabular}{|l|l|}
3021 & NMS \\
\end{tabular} & BOOT & Active & United States \\
\hline Madden (Steven) Ltd. & \begin{tabular}{|l|l|}
3144 & NMS \\
\end{tabular} & $\mathrm{SHOO}$ & Active & United States \\
\hline NIKE, Inc & \begin{tabular}{|l|l|}
3021 & NYS \\
\end{tabular} & NKE & Active & United States \\
\hline Phoenix Footwear Group, Inc. & \begin{tabular}{|l|l|l|}
3144 & ASE \\
\end{tabular} & PXG & Active & United States \\
\hline Rocky Brands Inc & 3143 NMS & RCKY & Active & United States \\
\hline Skechers U S A, Inc. & 3143 NYS & SKX & Active & United States \\
\hline Skins Inc & 3149 OTC & SKNN & Active & United States \\
\hline Timberland Co. (The) & 3143 NYS & TBL & Active & United States \\
\hline Weyco Group, Inc & 5139 NMS & WEYS & Active & United States \\
\hline Wolverine World Wide, Inc. (US) & 3149 NYS & WWW & Active & United States \\
\hline
\end{tabular}

\section{Stock Price Histories}

Following definition of companies within the specific NAICS code, Stock Price

Histories for each respective company within the NAICS code are downloaded from

Finance.Yahoo to determine year-to-year stock price changes. All company histories 
include an "Adjusted Close" which is adjusted for dividends and splits. Though the algorithm for this adjustment was not examined, it is accepted at face value since it is applied consistently to all companies, and the companies are categorized by industry (NAICS) mitigating possible distortions in the data. As noted by Hull (2007) "Dividend ratios vary systematically across industries due primarily to the comparable investment opportunities within an industry and to differences across industries" (Ch.18.1(1)). The adjusted close prices are then converted to a rolling 12-month average on a monthly basis to further eliminate year-end and seasonal distortions. The Adjusted Close 12-Month Rolling Average is then compared over 1-, 2-, 3-, and 5-year periods as shown in the Foot Locker example on Table 4 (dates 3/1/71 - 7/1/06 are hidden to conserve space): 
TABLE 4

\section{FOOT LOCKER STOCK PRICE CHANGES}

\begin{tabular}{|c|c|c|c|c|c|c|c|c|c|}
\hline $\begin{array}{l}\text { Company: } \\
\text { NAICS: } \\
\text { Monthly Pr } \\
\text { Date Acces } \\
\text { http://finand }\end{array}$ & $\begin{array}{l}\text { ices } \\
\text { ssed: } \\
\text { ce.yahoo }\end{array}$ & $\begin{array}{r}\text { Foot Lo } \\
31621 \\
1 / 2 / 70 \text { - } \\
9 / 14 / 2 \\
. c o m / q /\end{array}$ & $\begin{array}{l}\text { cker In } \\
\text { 9/14/0 } \\
2007 \\
\text { hp?s=F }\end{array}$ & $\begin{array}{l}7 \\
F L \& a=0\end{array}$ & $00 \& b=2 \& c$ & $=1970 \& d=0$ & $08 \& e=14 \&$ & $f=2007 \& g=m$ & \\
\hline Date & Open & High & Low & Close & Volume & Adj Close* & $\begin{array}{l}\text { Adj Close } \\
12 \text { mo } \\
\text { Rolling } \\
\text { Average }\end{array}$ & Comparison & \begin{tabular}{|l|} 
Rolling \\
Average \\
Percent \\
Change \\
\end{tabular} \\
\hline $9 / 4 / 2007$ & 16.78 & 16.86 & 15.91 & 16.49 & 998700 & 16.49 & $\succ 21.14$ & 41 year & $-7.99 \%$ \\
\hline $8 / 1 / 2007$ & 18.42 & 18.49 & 14.63 & 16.71 & 2315400 & 16.71 & 21.83 & 32 year & $-14.81 \%$ \\
\hline $7 / 2 / 2007$ & 21.72 & 23.6 & 18.21 & 18.56 & 2242400 & 18.56 & 22.41 & 3 year & $-4.75 \%$ \\
\hline $6 / 1 / 2007$ & 21.9 & 22.8 & 20.78 & 21.8 & 2086400 & 21.67 & 23.08 & 85 year & $58.79 \%$ \\
\hline $5 / 1 / 2007$ & 23.77 & 24.4 & 20.74 & 21.94 & 2062000 & 21.81 & 23.26 & & \\
\hline $4 / 2 / 2007$ & 23.55 & 24.72 & 23.04 & 23.79 & 1608100 & 23.65 & 23.41 & & \\
\hline $3 / 1 / 2007$ & 22.46 & 24.78 & 21.28 & 23.55 & 2054800 & 23.29 & 23.33 & & \\
\hline $2 / 1 / 2007$ & 22.54 & 23.47 & 22.24 & 22.72 & 1682700 & 22.47 & 23.32 & & \\
\hline $1 / 3 / 2007$ & 22.06 & 22.66 & 21.1 & 22.44 & 2557800 & 22.19 & 23.32 & & \\
\hline 12/1/2006 & 23.03 & 23.71 & 21.6 & 21.93 & 1809000 & 21.56 & 23.32 & & \\
\hline $11 / 1 / 2006$ & 23.19 & 24.92 & 22.21 & 22.9 & 2709500 & 22.52 & 23.43 & & \\
\hline $10 / 2 / 2006$ & 25 & 25.89 & 22.8 & 23.19 & 2826500 & 22.8 & 23.31 & & \\
\hline $9 / 1 / 2006$ & 24.1 & 25.55 & 22.34 & 25.25 & 2761100 & 24.74 & 22.98 & & \\
\hline $8 / 1 / 2006$ & 27 & 27.1 & 22.5 & 24.1 & 2597800 & 23.61 & 22.68 & & \\
\hline $2 / 1 / 1971$ & 39.5 & 48.13 & 38.5 & 47.63 & 209400 & 3.28 & 2.37 & & \\
\hline $1 / 4 / 1971$ & 36.5 & 40 & 35.5 & 39.75 & 107000 & 2.74 & 2.28 & & \\
\hline $12 / 1 / 1970$ & 35.13 & 37.88 & 35.13 & 36.5 & 66700 & 2.49 & 2.24 & & \\
\hline $11 / 2 / 1970$ & 33 & 35 & 32.25 & 34.88 & 57500 & 2.38 & & & \\
\hline $10 / 1 / 1970$ & 33.38 & 35.25 & 31.87 & 33 & 70100 & 2.26 & & & \\
\hline $9 / 1 / 1970$ & 35.25 & 35.38 & 32.75 & 33.38 & 47000 & 2.26 & & & \\
\hline $8 / 3 / 1970$ & 31.87 & 35.5 & 30.12 & 35.38 & 48400 & 2.4 & & & \\
\hline $7 / 1 / 1970$ & 29.25 & 32.75 & 28.75 & 31.87 & 53300 & 2.16 & & & \\
\hline $6 / 1 / 1970$ & 29.5 & 31.37 & 28.12 & 29.25 & 42100 & 1.96 & & & \\
\hline $5 / 1 / 1970$ & 31.87 & 31.87 & 25.37 & 29.5 & 69100 & 1.98 & & & \\
\hline 4/1/1970 & 35 & 35.63 & 29.12 & 32.13 & 49200 & 2.15 & & & \\
\hline $3 / 2 / 1970$ & 34.13 & 35.88 & 32.63 & 34.88 & 61200 & 2.32 & & & \\
\hline $2 / 2 / 1970$ & 33.63 & 36 & 32.88 & 34.13 & 94200 & 2.27 & & & \\
\hline $1 / 2 / 1970$ & 37.75 & \begin{tabular}{|l|}
38.88 \\
\end{tabular} & 33.63 & 33.63 & 75900 & 2.23 & & & \\
\hline
\end{tabular}


Table 4 demonstrates the method used to determine the rolling average changes in stock price. The average adjusted close price from 10/06 - 9/07 =21.14 reflected as "Adj close 12 mo Rolling Average” for 9/4/2007. This figure relative to the 9/1/2006 “Adj close 12 mo Rolling Average" (22.98) gives a negative 7.99\% 1-year change in stock price $[(21.14 / 22.98-1) \%$ with no rounding difference]. It can be further noted as shown in the period $1 / 2 / 1970-11 / 2 / 1970$ that the first year of adjusted close prices are not included in the rolling average since $<12$ months would be represented in that average. A minimum of two years of stock price history is required to be included in this study, 1 year to define the first 1 -year adjusted rolling average, then a $2^{\text {nd }}$ year for the comparison.

Once the stock price changes are consolidated for all companies in the NAICS category under consideration, individual company performance is then compared on a side-to-side basis as in Table 5 (Note: missing cells due to insufficient stock price history in the US Exchange): 
TABLE 5

MONTHLY AVERAGE ANNUAL STOCK PRICE CHANGE BY COMPANY

\begin{tabular}{|c|c|c|c|c|c|}
\hline & \multicolumn{5}{|c|}{$\begin{array}{l}\text { Prim NAICS } 31621 \\
\text { Footwear Manufacturing } \\
\text { US Exchanges Only } \\
12 \text { month rolling average year to year adjusted stock price comparison } \\
(\% \text { change }=(\mathrm{A} / \mathrm{B}-1) \%)\end{array}$} \\
\hline & $A$ & $9 / 07-$ & $9 / 07-$ & $9 / 07-$ & $9 / 07-$ \\
\hline & $\mathrm{B}$ & $9 / 06$ & $9 / 05$ & $9 / 04$ & $9 / 02$ \\
\hline & Company & $1 \mathrm{yr}$ & $2 \mathrm{yr}$ & $3 y r$ & $5 \mathrm{yr}$ \\
\hline 1 & Barry (R.G.) Corp. & $41 \%$ & $105 \%$ & $197 \%$ & $71 \%$ \\
\hline 2 & Brown Shoe Co., Inc. & $32 \%$ & $92 \%$ & $82 \%$ & $249 \%$ \\
\hline 3 & Cole (Kenneth) Productions, Inc. & $-4 \%$ & $-14 \%$ & $-19 \%$ & $24 \%$ \\
\hline 4 & Crocs Inc & & & & \\
\hline 5 & Deckers Outdoor Corp. & $118 \%$ & $136 \%$ & $208 \%$ & $1563 \%$ \\
\hline 6 & Foot Locker, Inc. & $-8 \%$ & $-15 \%$ & $-5 \%$ & $59 \%$ \\
\hline 7 & Iconix Brand Group Inc & $50 \%$ & $22 \%$ & $701 \%$ & $652 \%$ \\
\hline 8 & K-Swiss, Inc & $-3 \%$ & $-5 \%$ & $32 \%$ & $195 \%$ \\
\hline 9 & LaCrosse Footwear, Inc. & $33 \%$ & $45 \%$ & $113 \%$ & $425 \%$ \\
\hline 10 & Madden (Steven) Ltd. & $16 \%$ & $158 \%$ & $152 \%$ & $213 \%$ \\
\hline 11 & NIKE, Inc & $30 \%$ & $31 \%$ & $53 \%$ & $114 \%$ \\
\hline 12 & Phoenix Footwear Group, Inc. & $-26 \%$ & $-38 \%$ & $-57 \%$ & \\
\hline 13 & Rocky Brands Inc & $-35 \%$ & $-52 \%$ & $-36 \%$ & $124 \%$ \\
\hline 14 & Skechers U S A, Inc. & $38 \%$ & $107 \%$ & $160 \%$ & $79 \%$ \\
\hline 15 & Skins Inc & & & & \\
\hline 16 & Timberland Co. (The) & $-14 \%$ & $-22 \%$ & $-8 \%$ & $43 \%$ \\
\hline 17 & Weyco Group, Inc & $25 \%$ & $30 \%$ & $63 \%$ & $168 \%$ \\
\hline 18 & Wolverine World Wide, Inc. (US) & $20 \%$ & $34 \%$ & $86 \%$ & $180 \%$ \\
\hline & Count & 16 & 16 & 16 & 15 \\
\hline & Median & $22 \%$ & $30 \%$ & $73 \%$ & $168 \%$ \\
\hline & Mean & $20 \%$ & $39 \%$ & $108 \%$ & $277 \%$ \\
\hline & Standard Deviation & $36 \%$ & $64 \%$ & $178 \%$ & $391 \%$ \\
\hline
\end{tabular}

Finally, its distance in standard deviations from the mean determines the relative ranking of each company in each NAICS group (Appendix G). As shown in Table 6, Deckers ranks first in NAICS 31621 for the 1-, 2-, and 5-year comparisons with Iconix 
highest in the 3-year comparison while Rocky Brands ranks lowest in the 1-, 2-, and 3year comparisons with Timberland coming in lowest in the 5-year comparison:

TABLE 6

\section{COMPANY PERFORMANCE COMPARISONS}

\begin{tabular}{|c|c|c|c|c|}
\hline \multicolumn{5}{|c|}{$\begin{array}{l}\text { Prim NAICS } 31621 \\
\text { Footwear Manufacturing } \\
\text { US Exchanges Only } \\
12 \text { month rolling average year to year adjusted stock price comparison } \\
(\% \text { change }=(\mathrm{A} / \mathrm{B}-1) \%)\end{array}$} \\
\hline $\mathrm{A}$ & $9 / 07-$ & $9 / 07-$ & $9 / 07-$ & $9 / 07-$ \\
\hline$B$ & $9 / 06$ & $9 / 05$ & $9 / 04$ & $9 / 02$ \\
\hline Company & $1 \mathrm{yr}$ & $2 \mathrm{yr}$ & $3 \mathrm{yr}$ & $5 \mathrm{yr}$ \\
\hline \multicolumn{5}{|c|}{ Standard Deviation from Mean } \\
\hline Barry (R.G.) Corp. & 0.60 & 1.04 & 0.50 & -0.53 \\
\hline Brown Shoe Co., Inc. & 0.33 & 0.84 & -0.14 & -0.07 \\
\hline Cole (Kenneth) Productions, Inc. & -0.63 & -0.81 & -0.71 & -0.65 \\
\hline \multicolumn{5}{|l|}{ Crocs Inc } \\
\hline Deckers Outdoor Corp. & 2.71 & 1.53 & 0.56 & 3.28 \\
\hline Foot Locker, Inc. & -0.76 & -0.83 & -0.63 & -0.56 \\
\hline Iconix Brand Group Inc & 0.83 & -0.26 & 3.33 & 0.96 \\
\hline K-Swiss, Inc & -0.61 & -0.68 & -0.42 & -0.21 \\
\hline LaCrosse Footwear, Inc. & 0.37 & 0.10 & 0.03 & 0.38 \\
\hline Madden (Steven) Ltd. & -0.09 & 1.87 & 0.25 & -0.17 \\
\hline NIKE, Inc & 0.28 & -0.12 & -0.30 & -0.42 \\
\hline Phoenix Footwear Group, Inc. & -1.25 & -1.19 & -0.93 & \\
\hline Rocky Brands Inc & -1.50 & -1.41 & -0.80 & -0.39 \\
\hline Skechers U S A, Inc. & 0.50 & 1.07 & 0.29 & -0.51 \\
\hline \multicolumn{5}{|l|}{ Skins Inc } \\
\hline Timberland Co. (The) & -0.92 & -0.95 & -0.65 & -0.60 \\
\hline Weyco Group, Inc & 0.14 & -0.13 & -0.25 & -0.28 \\
\hline Wolverine World Wide, Inc. (US) & 0.02 & -0.07 & -0.12 & -0.25 \\
\hline
\end{tabular}

For purpose of determining correlation, the rankings of the companies in each

NAICS category will be broken into 3 levels of comparative performance: high, medium, and low. In this way, the relative performance of all companies under consideration will 
be standardized and high performers of all NAICS classifications can be compared against the medium and low performers of all NAICS classifications. The testing procedure for each hypothesis under consideration is now defined.

\section{$\underline{\text { Testing Procedures }}$}

Table 7 defines the Dependent and Independent variables and the measurement type for each hypothesis.

TABLE 7

TEST VARIABLES AND TYPE OF MEASURE

\begin{tabular}{|c|c|c|c|c|c|}
\hline & \multicolumn{5}{|c|}{ Hypotheses } \\
\hline & $\mathrm{H} 1 \mathrm{a}$ & $\mathrm{H} 1 \mathrm{~b}$ & $\mathrm{H} 2$ & $\mathrm{H} 3$ & $\mathrm{H} 4$ \\
\hline $\begin{array}{c}\text { Dependent } \\
\text { Variable }\end{array}$ & $\begin{array}{c}\text { Company } \\
\text { Performance }\end{array}$ & $\begin{array}{c}\text { Company } \\
\text { Performance }\end{array}$ & $\begin{array}{c}\text { Company } \\
\text { Performance }\end{array}$ & $\begin{array}{c}\text { Company } \\
\text { Performance }\end{array}$ & $\begin{array}{c}\text { Company } \\
\text { Performance }\end{array}$ \\
\hline \hline $\begin{array}{c}\text { Type of } \\
\text { Measure }\end{array}$ & Ordinal & Ordina! & Ordinal & Ordinal & Ordina! \\
\hline \multirow{2}{*}{$\begin{array}{c}\text { Independent } \\
\text { Variables }\end{array}$} & Identity & Priority & Financial & Financial & $\begin{array}{c}\text { Number } \\
\text { BSC } \\
\text { Measures }\end{array}$ \\
\cline { 2 - 6 } & Priority & Background & Nonfinancial & Nonfinancial & \\
\hline \hline & & & & & \\
\hline
\end{tabular}

A variety of possible statistical tests of association are considered in Table 8. Each test type is either considered an appropriate test for the Hypothesis under consideration or an inappropriate test with an explanation of the reason for rejection. 
TABLE 8

STATISTICAL TESTS OF ASSOCIATION FOR EACH HYPOTHESIS

\begin{tabular}{|c|c|c|c|c|c|}
\hline & \multicolumn{5}{|c|}{ Hypotheses } \\
\hline & H1a & H1b & $\mathrm{H} 2$ & $\mathrm{H} 3$ & $\mathrm{H} 4$ \\
\hline Type of Measure & Ordinal & Ordinal & Ordinal & Ordinal & Ordinal \\
\hline \multicolumn{6}{|c|}{ Association Tests } \\
\hline Parametric & $\begin{array}{c}\text { In appropriate - } \\
\text { minimum } \\
\text { interval } \\
\text { measure }\end{array}$ & $\begin{array}{c}\text { In appropriate - } \\
\text { minimum } \\
\text { interval } \\
\text { measure }\end{array}$ & $\begin{array}{c}\text { In appropriate - } \\
\text { minimum } \\
\text { interval } \\
\text { measure }\end{array}$ & $\begin{array}{l}\text { In appropriate - } \\
\text { minimum } \\
\text { interval } \\
\text { measure }\end{array}$ & $\begin{array}{c}\text { In appropriate - } \\
\text { minimum } \\
\text { interval } \\
\text { measure }\end{array}$ \\
\hline Non-Parametric & Appropriate & Appropriate & Appropriate & Appropriate & Appropriate \\
\hline $\begin{array}{l}\text { Wald-Wolfowitz } \\
\text { Runs Test }\end{array}$ & $\begin{array}{c}\text { In appropriate - } \\
\text { two-sample } \\
\text { test }\end{array}$ & $\begin{array}{c}\text { In appropriate - } \\
\text { two-sample } \\
\text { test }\end{array}$ & $\begin{array}{c}\text { In appropriate - } \\
\text { two-sample } \\
\text { test }\end{array}$ & $\begin{array}{c}\text { In appropriate - } \\
\text { two-sample } \\
\text { test }\end{array}$ & $\begin{array}{c}\text { In appropriate - } \\
\text { two-sample } \\
\text { test }\end{array}$ \\
\hline $\begin{array}{l}\text { Mann-Whitney or } \\
\text { Wilcoxon Test }\end{array}$ & $\begin{array}{c}\text { Inappropriate - } \\
\text { two-sample } \\
\text { test }\end{array}$ & $\begin{array}{c}\text { Inappropriate - } \\
\text { two-sample } \\
\text { test }\end{array}$ & $\begin{array}{c}\text { Inappropriate - } \\
\text { two-sample } \\
\text { test }\end{array}$ & $\begin{array}{c}\text { Inappropriate - } \\
\text { two-sample } \\
\text { test }\end{array}$ & $\begin{array}{c}\text { In appropriate - } \\
\text { two-sample } \\
\text { test }\end{array}$ \\
\hline $\begin{array}{l}\text { Kolmogoroy- } \\
\text { Smirnov Test }\end{array}$ & $\begin{array}{c}\text { Inappropriate - } \\
\text { two-sample } \\
\text { test }\end{array}$ & $\begin{array}{c}\text { Inappropriate - } \\
\text { two-sample } \\
\text { test }\end{array}$ & $\begin{array}{c}\text { Inappropriate - } \\
\text { two-sample } \\
\text { test }\end{array}$ & $\begin{array}{c}\text { Inappropriate - } \\
\text { two-sample } \\
\text { test }\end{array}$ & $\begin{array}{c}\text { In appropriate - } \\
\text { two-sample } \\
\text { test }\end{array}$ \\
\hline $\begin{array}{l}\text { Wilcoxon } \\
\text { Matched-pairs } \\
\text { Signed-ranks Test }\end{array}$ & $\begin{array}{c}\text { In appropriate - } \\
\text { two-sample } \\
\text { test }\end{array}$ & $\begin{array}{c}\text { Inappropriate - } \\
\text { two-sample } \\
\text { test }\end{array}$ & $\begin{array}{c}\text { Inappropriate - } \\
\text { two-sample } \\
\text { test }\end{array}$ & $\begin{array}{c}\text { Inappropriate - } \\
\text { two-sample } \\
\text { test }\end{array}$ & $\begin{array}{c}\text { In appropriate - } \\
\text { two-sample } \\
\text { test }\end{array}$ \\
\hline $\begin{array}{l}\text { Friedman's Rank } \\
\text { Test for k } \\
\text { Correlated } \\
\text { Samples }\end{array}$ & $\begin{array}{l}\text { Inappropriate - } \\
\text { repeated } \\
\text { measures test }\end{array}$ & $\begin{array}{l}\text { Inappropriate - } \\
\text { repeated } \\
\text { measures test }\end{array}$ & $\begin{array}{l}\text { In appropriate - } \\
\text { repeated } \\
\text { measures test }\end{array}$ & $\begin{array}{l}\text { Inappropriate - } \\
\text { repeated } \\
\text { measures test }\end{array}$ & $\begin{array}{l}\text { In appropriate - } \\
\text { repeated } \\
\text { measures test }\end{array}$ \\
\hline $\begin{array}{l}\text { Kruskal-Wallis } \\
\text { One-Way } \\
\text { Analysis of } \\
\text { Variance }\end{array}$ & Appropriate & Appropriate & Appropriate & Appropriate & Appropriate \\
\hline Chi-square Test & Appropriate & Appropriate & Appropriate & Appropriate & Appropriate \\
\hline
\end{tabular}

(Blalock, H. M., 1972; Howell, D. C. 2002)

Finally, each Hypothesis is considered relative to possible statistical tests of strength of association in Table 9: 
TABLE 9

\section{STATISTICAL TESTS OF STRENGTH OF ASSOCIATION FOR EACH HYPOTHESIS}

\begin{tabular}{|c|c|c|c|c|c|}
\hline & \multicolumn{5}{|c|}{ Hypotheses } \\
\hline & $\mathrm{H} 1 \mathrm{a}$ & H1b & $\mathrm{H} 2$ & $\mathrm{H3}$ & $\mathrm{H} 4$ \\
\hline $\begin{array}{c}\text { Type of } \\
\text { Measure } \\
\end{array}$ & Ordinal & Ordinal & Ordinal & Ordinal & Ordinal \\
\hline \multicolumn{6}{|c|}{ Strength of Asso ciation Tests } \\
\hline Cohen's Kappa & $\begin{array}{l}\text { In appropriate } \\
\text { maximum two } \\
\text { raters only }\end{array}$ & $\begin{array}{l}\text { In appropriate } \\
\text { maximum two } \\
\text { raters only }\end{array}$ & $\begin{array}{l}\text { In appropriate } \\
\text { maximum two } \\
\text { raters only }\end{array}$ & $\begin{array}{l}\text { In appropriate } \\
\text { maximum two } \\
\text { raters only }\end{array}$ & $\begin{array}{l}\text { In appropriate } \\
\text { maximum two } \\
\text { raters only }\end{array}$ \\
\hline Spearman's r & $\begin{array}{l}\text { Appropriate - } \\
\text { not necessary } \\
\text { to reject null }\end{array}$ & $\begin{array}{l}\text { Appropriate - } \\
\text { not necessary } \\
\text { to reject null }\end{array}$ & $\begin{array}{l}\text { Appropriate - } \\
\text { not necessary } \\
\text { to reject null }\end{array}$ & Appropriate & $\begin{array}{l}\text { Appropriate - } \\
\text { not necessary } \\
\text { to reject null }\end{array}$ \\
\hline Kendall's tau & $\begin{array}{l}\text { Appropriate - } \\
\text { not necessary } \\
\text { to reject null }\end{array}$ & $\begin{array}{l}\text { Appropriate - } \\
\text { not necessary } \\
\text { to reject null }\end{array}$ & $\begin{array}{l}\text { Appropriate - } \\
\text { not necessary } \\
\text { to reject null }\end{array}$ & Appropriate & $\begin{array}{l}\text { Appropriate - } \\
\text { not necessary } \\
\text { to reject null }\end{array}$ \\
\hline Gamma & $\begin{array}{l}\text { Appropriate - } \\
\text { not necessary } \\
\text { to reject null }\end{array}$ & $\begin{array}{l}\text { Appropriate - } \\
\text { not necessary } \\
\text { to reject null }\end{array}$ & $\begin{array}{l}\text { Appropriate - } \\
\text { not necessary } \\
\text { to reject null }\end{array}$ & Appropriate & $\begin{array}{l}\text { Appropriate - } \\
\text { not necessary } \\
\text { to reject null }\end{array}$ \\
\hline $\begin{array}{l}\text { Goodman and } \\
\text { Kruskal's tau }\end{array}$ & $\begin{array}{l}\text { Appropriate - } \\
\text { not necessary } \\
\text { to reject null }\end{array}$ & $\begin{array}{l}\text { Appropriate - } \\
\text { not necessary } \\
\text { to reject null }\end{array}$ & $\begin{array}{l}\text { Appropriate - } \\
\text { not necessary } \\
\text { to reject null }\end{array}$ & Appropriate & $\begin{array}{l}\text { Appropriate - } \\
\text { not necessary } \\
\text { to reject null }\end{array}$ \\
\hline $\begin{array}{l}\text { Cramers Phi } \\
\text { (Cramers V) }\end{array}$ & $\begin{array}{l}\text { Appropriate - } \\
\text { not necessary } \\
\text { to reject null }\end{array}$ & $\begin{array}{l}\text { Appropriate - } \\
\text { not necessary } \\
\text { to reject null }\end{array}$ & $\begin{array}{l}\text { Appropriate - } \\
\text { not necessary } \\
\text { to reject null }\end{array}$ & Appropriate & $\begin{array}{l}\text { Appropriate - } \\
\text { not necessary } \\
\text { to reject null }\end{array}$ \\
\hline \multicolumn{6}{|c|}{ (Blalock, H. M., 1972; Howell, D. C. 2002) } \\
\hline
\end{tabular}

The testing methodology proposed for each Hypothesis is now described in

further detail: 
$\mathrm{H}_{1}$ : Salience of business processes identified for measurement correlate to firm value as measured by Kruskal-Wallis one-way ANOVA.

$\mathrm{H}_{1 \mathrm{a}}$ : Identity business processes using the means results from $\mathrm{H}_{1}$ have a higher positive correlation to firm value than Priority business processes as measured by the Chi-Square Contingency Test of Association.

$\mathrm{H}_{1 \mathrm{~b}}$ : Priority business processes using the means results from $\mathrm{H}_{1}$ have a higher positive correlation to firm value than Background business processes as measured by the Chi-Square Contingency Test of Association.

Kruskal-Wallis one-way ANOVA is used to test against the null for $\mathrm{H}_{1}$, followed by the Chi-Square Contingency Test of Association using the means results from $\mathrm{H}_{1}$ to test against the null for $\mathrm{H}_{1 \mathrm{a}}$ and $\mathrm{H}_{1 \mathrm{~b}}$.

A hypothetical set of responses from 23 respondents (Table 10), summarized by group and rank (rate) in Table 11, is used to demonstrate the tests of these hypotheses. 
TABLE 10

HYPOTHETICAL RESPONSES SURVEY QUESTION 1

\begin{tabular}{|c|c|c|c|c|c|}
\hline \multirow{2}{*}{ Respondent } & \multirow{2}{*}{$\begin{array}{c}\text { Performance } \\
\text { Level }\end{array}$} & \multicolumn{4}{|c|}{ Rank Use (1 - 4) } \\
\hline & & Identity & Priority & Background & Mandated \\
\hline 1 & $\mathrm{H}$ & 1 & 2 & 4 & 3 \\
\hline 2 & $\mathrm{H}$ & 1 & 2 & 4 & 3 \\
\hline 3 & $\mathrm{H}$ & 1 & 2 & 4 & 3 \\
\hline 4 & $\mathrm{H}$ & 4 & 3 & 2 & 1 \\
\hline 5 & $\mathrm{H}$ & 2 & 1 & 3 & 4 \\
\hline 6 & $\mathrm{H}$ & 2 & 1 & 3 & 4 \\
\hline 7 & $\mathrm{H}$ & 1 & 2 & 4 & 3 \\
\hline 8 & $\mathrm{H}$ & 2 & 1 & 3 & 4 \\
\hline 9 & $\mathrm{H}$ & 2 & 1 & 3 & 4 \\
\hline 10 & $M$ & 1 & 2 & 4 & 3 \\
\hline 11 & $\mathrm{M}$ & 2 & 1 & 3 & 4 \\
\hline 12 & $M$ & 2 & 1 & 3 & 4 \\
\hline 13 & M & 4 & 3 & 2 & 1 \\
\hline 14 & $\mathrm{M}$ & 3 & 4 & 1 & 2 \\
\hline 15 & $M$ & 3 & 4 & 1 & 2 \\
\hline 16 & $\mathrm{~L}$ & 3 & 4 & 1 & 2 \\
\hline 17 & $\mathrm{~L}$ & 3 & 4 & 1 & 2 \\
\hline 18 & $\mathrm{~L}$ & 3 & 4 & 1 & 2 \\
\hline 19 & $\mathrm{~L}$ & 3 & 4 & 1 & 2 \\
\hline 20 & $\mathrm{~L}$ & 3 & 4 & 1 & 2 \\
\hline 21 & $\mathrm{~L}$ & 4 & 3 & 2 & 1 \\
\hline 22 & $\mathrm{~L}$ & 4 & 3 & 2 & 1 \\
\hline 23 & $\mathrm{~L}$ & 2 & 1 & 3 & 4 \\
\hline
\end{tabular}


TABLE 11

HYPOTHETICAL CASE -- RESPONSES BY PERFORMANCE

LEVEL AND RESPONSE RANK (RATE)

\begin{tabular}{|c|c|c|c|}
\hline Identity & \multicolumn{3}{|c|}{ Performance Level } \\
\hline Rank (rate) & $\mathbf{H}$ & $\mathbf{M}$ & $\mathbf{L}$ \\
\hline 1 & 4 & 1 & 0 \\
\hline 2 & 4 & 2 & 1 \\
\hline 3 & 0 & 2 & 5 \\
\hline 4 & 1 & 1 & 2 \\
\hline Total & 9 & 6 & 8 \\
\hline Priority & \multicolumn{3}{|c|}{ Performance Level } \\
\hline Rank & $\mathbf{H}$ & M & $\mathbf{L}$ \\
\hline 1 & 4 & 2 & 1 \\
\hline 2 & 4 & 1 & 0 \\
\hline 3 & 1 & 1 & 2 \\
\hline 4 & 0 & 2 & 5 \\
\hline Total & 9 & 6 & 8 \\
\hline Background & \multicolumn{3}{|c|}{ Performance Level } \\
\hline Rank (rate) & $\mathbf{H}$ & $\mathbf{M}$ & $\mathbf{L}$ \\
\hline 1 & 0 & 2 & 5 \\
\hline 2 & 1 & 1 & 2 \\
\hline 3 & 4 & 2 & 1 \\
\hline 4 & 4 & 1 & 0 \\
\hline Total & 9 & 6 & 8 \\
\hline
\end{tabular}

The first task is to confirm that High, Middle and Low performing companies do in fact rate Identity, Priority and Background processes differently (Mandated processes are also rated in the survey, but are not included in the test since mandated processes are not a component of the Hypotheses). The Kruskal-Wallis one-way ANOVA is accomplished in a series of four steps. The initial data is placed in a matrix for each salience process (Salience Identity demonstrated in Table 11) showing the dependent 
variable (company performance) in columns and the independent variable (Salience rating) in rows. The salience ratings then are organized in a matrix to rank all scores without regard to group membership in increasing rank from Rate 1 to 4 (like rates are "tied" and are therefore averaged) to determine a mean ranking level for each rate type (1 -4) as shown in step two, Table 12. 
TABLE 12

KRUSKAL-WALLIS EXAMPLE STEPS 1 AND 2 (IDENTITY)

\begin{tabular}{|c|c|c|c|c|}
\hline \multirow{2}{*}{$\begin{array}{c}\text { Step } 1 \\
\text { Raw } \\
\text { Measures }\end{array}$} & \multicolumn{4}{|c|}{ Performance Level } \\
\hline & Respondent & High & Medium & Low \\
\hline \multirow{9}{*}{ 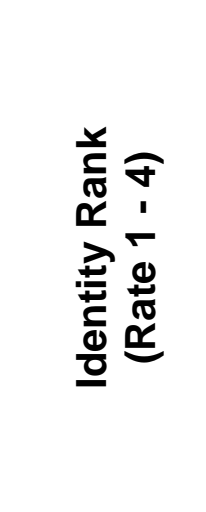 } & $1(H, M, L)$ & 1 & 1 & 3 \\
\hline & 2 & 1 & 2 & 3 \\
\hline & 3 & 1 & 2 & 3 \\
\hline & 4 & 4 & 4 & 3 \\
\hline & 5 & 2 & 3 & 3 \\
\hline & 6 & 2 & 3 & 4 \\
\hline & 7 & 1 & & 4 \\
\hline & 8 & 2 & & 2 \\
\hline & 9 & 2 & & \\
\hline \multirow[t]{5}{*}{ Step 2} & Salience Rank & Count & Tied & \\
\hline & 1 & 1 & & \\
\hline & 1 & 2 & & \\
\hline & 1 & 3 & & \\
\hline & 1 & 4 & & \\
\hline \multirow{4}{*}{$\frac{\frac{n}{n}}{\frac{n}{\sqrt{0}}}$} & 1 & 5 & 3 & Average \\
\hline & 2 & 6 & & \\
\hline & 2 & 7 & & \\
\hline & 2 & 8 & & \\
\hline \multirow{15}{*}{ 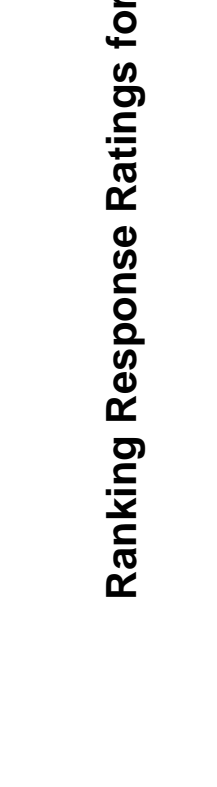 } & 2 & 9 & & \\
\hline & 2 & 10 & & \\
\hline & 2 & 11 & & \\
\hline & 2 & 12 & 9 & Average \\
\hline & 3 & 13 & & \\
\hline & 3 & 14 & & \\
\hline & 3 & 15 & & \\
\hline & 3 & 16 & & \\
\hline & 3 & 17 & & \\
\hline & 3 & 18 & & \\
\hline & 3 & 19 & 16 & Average \\
\hline & 4 & 20 & & \\
\hline & 4 & 21 & & \\
\hline & 4 & 22 & & \\
\hline & 4 & 23 & 21.5 & Average \\
\hline
\end{tabular}


In this test, Rank (rate) 1 is considered by respondent to be most used type of process and Rank (rate) 4 is least used, so the lower mean number reflects more often used process types.

Once the mean ranking levels for each rated salience process are identified, the raw data rates $(1-4)$ are replaced by the ranked means for those tied rates in the raw data matrix as exhibited in Step 3, followed by the Kruksall-Wallis one-way ANOVA calculation in Step 4 Table 13, where the H statistic is treated as though it is a value of chi-square with degrees of freedom equal to $\mathrm{k}-1$ where $\mathrm{k}$ is the number of groups. 
TABLE 13

KRUSKAL-WALLIS EXAMPLE STEPS 3 AND 4 (IDENTITY)

\begin{tabular}{|c|c|c|c|c|c|}
\hline \multicolumn{2}{|c|}{ Step 3} & \multicolumn{4}{|c|}{ Performance Level } \\
\hline & Respondent & High & Medium & Low & Total \\
\hline & $1(H, M, L)$ & 3.0 & 3.0 & 16 & \\
\hline & 2 & 3.0 & 9.0 & 16 & \\
\hline & 3 & 3.0 & 9.0 & 16 & \\
\hline & 4 & 21.5 & 21.5 & 16 & \\
\hline & 5 & 9.0 & 16 & 16 & \\
\hline & 6 & 9.0 & 16 & 21.5 & \\
\hline & 7 & 3.0 & & 21.5 & \\
\hline & 8 & 9.0 & & 9.0 & \\
\hline & 9 & 9.0 & & & \\
\hline & Count & 9 & 6 & 8 & 23 \\
\hline & sum of ranks & 69.5 & 74.5 & 132.0 & 276 \\
\hline & average of ranks & 7.72 & 12.42 & 16.50 & 12.00 \\
\hline \multirow[t]{5}{*}{ Step 4} & $\begin{array}{c}\text { Ranked Measures } \\
\text { (example) }\end{array}$ & \multicolumn{4}{|c|}{ Performance Level } \\
\hline & & High & Medium & Low & All \\
\hline & counts $(n)$ & 9 & 6 & 8 & 23 \\
\hline & sums $(\mathrm{R})$ & 69.5 & 74.5 & 132 & 276 \\
\hline & means & 7.72 & 12.42 & 16.50 & 12.00 \\
\hline$H=$ & $\begin{array}{l}12 /[N(N+1)] \Sigma_{i=1}^{k} R^{2} \\
\text { where } \\
k=\text { number of grol } \\
n_{i}=\text { number of obs } \\
R_{i}=\text { sum of the rar } \\
N=\Sigma n_{i}=\text { total sam }\end{array}$ & $\begin{array}{l}\text { s in grou } \\
\text { oup i }\end{array}$ & & & \\
\hline $\begin{array}{l}\mathrm{H}= \\
\mathrm{H}=\end{array}$ & \multicolumn{5}{|c|}{$\begin{array}{c}12 /[23(23+1)]\left(69.5^{2} / 9+74.5^{2} / 6+132^{2} / 8\right)-3(23+1) \\
7.12\end{array}$} \\
\hline$d f=$ & $\begin{array}{l}3-1=2 \\
7.12>5.991\end{array}$ & \multicolumn{4}{|c|}{$\begin{array}{l}\text { Chi Square at } 0.05=5.991 \\
\text { Reject null at } .05\end{array}$} \\
\hline
\end{tabular}


In this Example, the null hypothesis -- no difference in ratings of use of salience Identity by High, Middle and Low performers -- is rejected at a 0.05 significance level.

For the purpose of demonstration of reasonableness in this hypothetical case Tables $14-17$ display Priority and Background responses and results using the initial hypothetical responses from Table 10. 
TABLE 14

KRUSKAL-WALLIS EXAMPLE STEPS 1 AND 2 (PRIORITY)

\begin{tabular}{|c|c|c|c|c|}
\hline \multirow{2}{*}{$\begin{array}{c}\text { Step } 1 \\
\text { Raw } \\
\text { Measures }\end{array}$} & \multicolumn{4}{|c|}{ Performance Level } \\
\hline & Respondent & High & Medium & Low \\
\hline \multirow{9}{*}{ 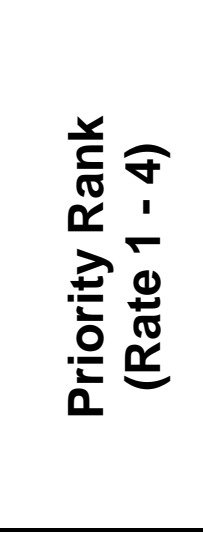 } & $1(H, M, L)$ & 2 & 2 & 4 \\
\hline & 2 & 2 & 1 & 4 \\
\hline & 3 & 2 & 1 & 4 \\
\hline & 4 & 3 & 3 & 4 \\
\hline & 5 & 1 & 4 & 4 \\
\hline & 6 & 1 & 4 & 3 \\
\hline & 7 & 2 & & 3 \\
\hline & 8 & 1 & & 1 \\
\hline & 9 & 1 & & \\
\hline \multirow[t]{24}{*}{ Step 2} & Salience Rank & Count & Tied & \\
\hline & 1 & 1 & & \\
\hline & 1 & 2 & & \\
\hline & 1 & 3 & & \\
\hline & 1 & 4 & & \\
\hline & 1 & 5 & & \\
\hline & 1 & 6 & & \\
\hline & 1 & 7 & 4 & Average \\
\hline & 2 & 8 & & \\
\hline & 2 & 9 & & \\
\hline & 2 & 10 & & \\
\hline & 2 & 11 & & \\
\hline & 2 & 12 & 10 & Average \\
\hline & 3 & 13 & & \\
\hline & 3 & 14 & & \\
\hline & 3 & 15 & & \\
\hline & 3 & 16 & 14.5 & Average \\
\hline & 4 & 17 & & \\
\hline & 4 & 18 & & \\
\hline & 4 & 19 & & \\
\hline & 4 & 20 & & \\
\hline & 4 & 21 & & \\
\hline & 4 & 22 & & \\
\hline & 4 & 23 & 20 & Average \\
\hline
\end{tabular}


TABLE 15

KRUSKAL-WALLIS EXAMPLE STEPS 3 AND 4 (PRIORITY)

\begin{tabular}{|c|c|c|c|c|c|}
\hline \multicolumn{2}{|c|}{ Step 3} & \multicolumn{4}{|c|}{$\begin{array}{l}\text { Performance Level } \\
\text { Ranked Measures (example) }\end{array}$} \\
\hline \multicolumn{2}{|r|}{ Respondent } & High & Medium & Low & Total \\
\hline \multicolumn{2}{|r|}{$1(H, M, L)$} & 10.0 & 10.0 & 20 & \\
\hline \multicolumn{2}{|r|}{2} & 10.0 & 4.0 & 20 & \\
\hline \multicolumn{2}{|r|}{3} & 10.0 & 4.0 & 20 & \\
\hline \multicolumn{2}{|r|}{4} & 14.5 & 14.5 & 20 & \\
\hline \multicolumn{2}{|r|}{5} & 4.0 & 20 & 20 & \\
\hline \multicolumn{2}{|r|}{6} & 4.0 & 20 & 14.5 & \\
\hline \multicolumn{2}{|r|}{7} & 10.0 & & 14.5 & \\
\hline \multicolumn{2}{|r|}{8} & 4.0 & & 4.0 & \\
\hline \multicolumn{2}{|r|}{9} & 4.0 & & & \\
\hline \multicolumn{2}{|r|}{ Count } & 9 & 6 & 8 & 23 \\
\hline \multicolumn{2}{|r|}{ sum of ranks } & 70.5 & 72.5 & 133.0 & 276 \\
\hline \multicolumn{2}{|r|}{$\begin{array}{l}\text { average of } \\
\text { ranks }\end{array}$} & 7.83 & 12.08 & 16.63 & 12.00 \\
\hline \multicolumn{2}{|c|}{ Step 4} & \multicolumn{4}{|c|}{ Performance Level } \\
\hline & & High & Medium & Low & All \\
\hline & counts (n) & 9 & 6 & 8 & 23 \\
\hline & sums (R) & 70.5 & 72.5 & 133 & 276 \\
\hline & means & 7.83 & 12.08 & 16.63 & 12.00 \\
\hline $\mathrm{H}=$ & $\begin{array}{l}12 /[N(N+1)] \Sigma^{k}= \\
\text { where } \\
k=\text { number of } \mathrm{g} \\
n_{i}=\text { number of } \\
R_{i}=\text { sum of the } \\
N=\sum n_{i}=\text { total } s \\
\text { size }\end{array}$ & $\begin{array}{l}\text { oups } \\
\text { oservatio } \\
\text { anks in g } \\
\text { ample }\end{array}$ & $\begin{array}{l}\text { in group i } \\
\text { up i }\end{array}$ & & \\
\hline $\begin{array}{l}H= \\
H= \\
d f=\end{array}$ & \multicolumn{5}{|c|}{$\begin{array}{c}12 /[23(23+1)]\left(70.5^{2} / 9+72.5^{2} / 6+133^{2} / 8\right)-3(23+1) \\
7.12\end{array}$} \\
\hline
\end{tabular}


TABLE 16

KRUSKAL-WALLIS EXAMPLE STEPS 1 AND 2 (BACKGROUND)

\begin{tabular}{|c|c|c|c|c|}
\hline \multirow{2}{*}{$\begin{array}{c}\text { Step } 1 \\
\text { Raw } \\
\text { Measures }\end{array}$} & \multicolumn{4}{|c|}{ Performance Level } \\
\hline & Respondent & High & Medium & Low \\
\hline \multirow{9}{*}{ 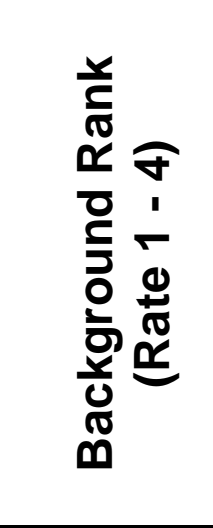 } & $1(H, M, L)$ & 4 & 4 & 1 \\
\hline & 2 & 4 & 3 & 1 \\
\hline & 3 & 4 & 3 & 1 \\
\hline & 4 & 2 & 2 & 1 \\
\hline & 5 & 3 & 1 & 1 \\
\hline & 6 & 3 & 1 & 2 \\
\hline & 7 & 4 & & 2 \\
\hline & 8 & 3 & & 3 \\
\hline & 9 & 3 & & \\
\hline \multirow[t]{24}{*}{ Step 2} & Salience Rank & Count & Tied & \\
\hline & 1 & 1 & & \\
\hline & 1 & 2 & & \\
\hline & 1 & 3 & & \\
\hline & 1 & 4 & & \\
\hline & 1 & 5 & & \\
\hline & 1 & 6 & & \\
\hline & 1 & 7 & 4 & Average \\
\hline & 2 & 8 & & \\
\hline & 2 & 9 & & \\
\hline & 2 & 10 & & \\
\hline & 2 & 11 & 9.5 & Average \\
\hline & 3 & 12 & & \\
\hline & 3 & 13 & & \\
\hline & 3 & 14 & & \\
\hline & 3 & 15 & & \\
\hline & 3 & 16 & & \\
\hline & 3 & 17 & & \\
\hline & 3 & 18 & 15 & Average \\
\hline & 4 & 19 & & \\
\hline & 4 & 20 & & \\
\hline & 4 & 21 & & \\
\hline & 4 & 22 & & \\
\hline & 4 & 23 & 21 & Average \\
\hline
\end{tabular}


TABLE 17

KRUSKAL-WALLIS EXAMPLE STEPS 3 AND 4 (BACKGROUND)

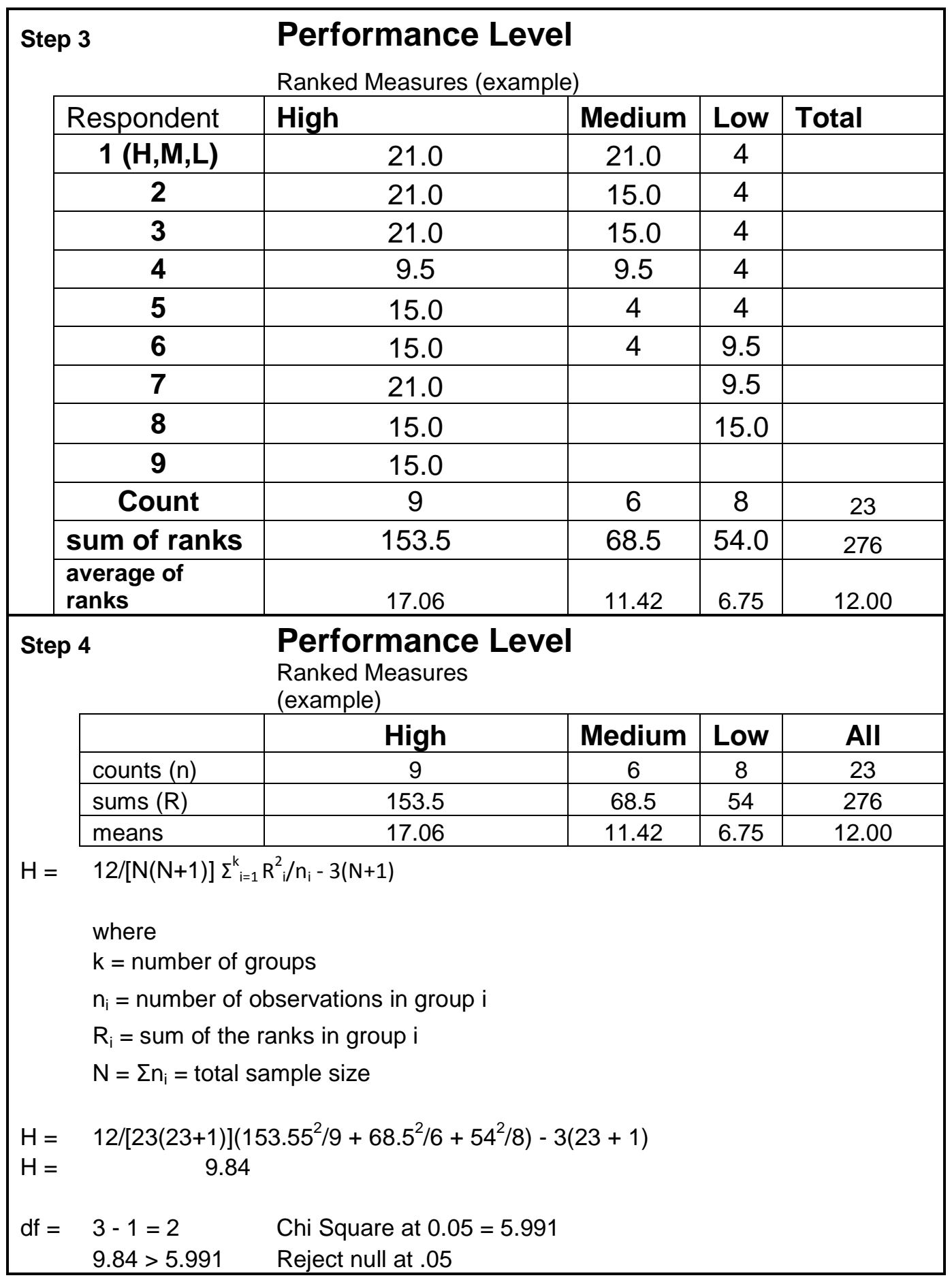


A summary of the results in Table 18 shows that in this hypothetical case, for all three salience types, the null hypothesis (no difference in rating of salience types exists for High, Middle and Low performers) is rejected at a 0.05 level of significance.

TABLE 18

KRUSKAL-WALLIS EXAMPLE RESULTS (IDENTITY, PRIORITY, BACKGROUND)

\begin{tabular}{|c|c|c|c|c|}
\hline \multicolumn{5}{|c|}{$\begin{array}{l}\text { Performance Level } \\
\text { Ranked Measures (example) }\end{array}$} \\
\hline \multicolumn{5}{|c|}{ Identity } \\
\hline & High & Medium & Low & All \\
\hline counts $(n)$ & 9 & 6 & 8 & 23 \\
\hline sums $(R)$ & 69.5 & 74.5 & 132 & 276 \\
\hline means & 7.72 & 12.42 & 16.50 & 12.00 \\
\hline $\begin{array}{l}H= \\
H= \\
d f=\end{array}$ & $\begin{array}{l}12 /[N(N+1)] \\
3-1=2 \\
7.12>5.991\end{array}$ & \multicolumn{3}{|c|}{$\begin{array}{l}\text { Chi Square at } 0.05= \\
5.991 \\
\text { Reject null at } .05\end{array}$} \\
\hline \multicolumn{5}{|c|}{ Priority } \\
\hline & High & Medium & Low & All \\
\hline counts $(n)$ & 9 & 6 & 8 & 23 \\
\hline sums $(R)$ & 70.5 & 72.5 & 133 & 276 \\
\hline means & 7.83 & 12.08 & 16.63 & 12.00 \\
\hline $\begin{array}{l}H= \\
H= \\
d f=\end{array}$ & $\begin{array}{l}12 /[N(N+1)] \\
3-1=2 \\
7.12>5.991\end{array}$ & $\begin{array}{l}/ \mathrm{n}_{\mathrm{i}}-3(\mathrm{~N}+1) \\
\text { Chi Square a } \\
5.991 \\
\text { Reject null a }\end{array}$ & $5=$ & \\
\hline \multicolumn{5}{|c|}{ Background } \\
\hline & High & Medium & Low & All \\
\hline counts $(n)$ & 9 & 6 & 8 & 23 \\
\hline sums $(R)$ & 153.5 & 68.5 & 54 & 276 \\
\hline means & 17.06 & 11.42 & 6.75 & 12.00 \\
\hline $\begin{array}{l}H= \\
H= \\
d f=\end{array}$ & $\begin{array}{l}12 /[N(N+1)] \Sigma \\
3-1=2 \\
9.84>5.991\end{array}$ & $\begin{array}{l}/ \mathrm{n}_{\mathrm{i}}-3(\mathrm{~N}+1) \\
\text { Chi Square a } \\
5.991 \\
\text { Reject null a }\end{array}$ & & \\
\hline
\end{tabular}


The final step is to use the Chi-Square Contingency Test of Association to reject the null for the two specific sub-hypotheses as demonstrated in this hypothetical case using The Kruskal-Wallis group means.

$\mathrm{H}_{1 \mathrm{a}}$ : Identity business processes using the means results from $\mathrm{H}_{1}$ have a higher positive correlation to firm value than Priority business processes as measured by the Chi-Square Contingency Test of Association.

TABLE 19

CHI-SQUARE CONTINGENCY TEST EXAMPLE $\mathrm{H}_{1 \mathrm{~A}}$

\begin{tabular}{|c|c|c|c|c|}
\hline \multicolumn{5}{|c|}{ Identity vs. Priority (H1a) } \\
\hline Observed & High & Medium & Low & Total \\
\hline Identity & 7.72 & 12.42 & 16.5 & 36.64 \\
\hline Priority & 7.83 & 12.08 & 16.63 & 36.54 \\
\hline Total & 15.55 & 24.5 & 33.13 & 73.18 \\
\hline \multicolumn{5}{|l|}{ Expected } \\
\hline Identity & 7.79 & 12.27 & 16.59 & \\
\hline Priority & 7.76 & 12.23 & 16.54 & \\
\hline Observed & Expected & $O-E$ & $(\mathrm{O}-\mathrm{E})^{\wedge} 2$ & $(O-E)^{\wedge} 2 / E$ \\
\hline 7.72 & 7.79 & -0.07 & 0.004 & 0.000553144 \\
\hline 7.83 & 7.76 & 0.07 & 0.004 & 0.000554658 \\
\hline 12.42 & 12.27 & 0.15 & 0.023 & 0.001914834 \\
\hline 12.08 & 12.23 & -0.15 & 0.023 & 0.001920074 \\
\hline 16.5 & 16.59 & -0.09 & 0.008 & 0.000462999 \\
\hline 16.63 & 16.54 & 0.09 & 0.008 & 0.000464266 \\
\hline \multirow[t]{2}{*}{$\mathrm{df}=2$} & & $0.05=5.991$ & & $0.00586997 €$ \\
\hline & \multicolumn{2}{|c|}{$0.00587<5.991$} & \multicolumn{2}{|c|}{ Cannot reject Null at 0.05} \\
\hline
\end{tabular}




$$
\mathrm{H}_{1 \mathrm{~b}} \text { : Priority business processes using the means results from } \mathrm{H}_{1} \text { have a higher }
$$

positive correlation to firm value than Background business processes as measured by the Chi-Square Contingency Test of Association.

TABLE 20

CHI-SQUARE CONTINGENCY TEST EXAMPLE $\mathrm{H}_{1 \mathrm{~B}}$

\begin{tabular}{|c|c|c|c|c|}
\hline \multicolumn{5}{|c|}{ Priority vs. Background (H1b) } \\
\hline Observed & High & Medium & Low & Total \\
\hline Priority & 7.83 & 12.08 & 16.63 & 36.54 \\
\hline Background & 17.06 & 11.42 & 6.75 & 35.22 \\
\hline Total & 24.89 & 23.50 & 23.38 & 71.76 \\
\hline Expected & & & & \\
\hline Identity & 12.67 & 11.97 & 11.90 \\
\hline Priority & 12.22 & 11.53 & 11.47 & \\
\hline \multicolumn{5}{|c|}{} \\
\hline Observed & Expected & $0-\mathrm{E}$ & $(\mathrm{O}-\mathrm{E})^{\wedge} 2$ & $(\mathrm{O}-\mathrm{E})^{\wedge} 2 / \mathrm{E}$ \\
\hline 7.833333333 & 12.67 & -4.84 & 23.425 & 1.848363203 \\
\hline 17.05555556 & 12.22 & 4.84 & 23.425 & 1.917603938 \\
\hline 12.08333333 & 11.97 & 0.12 & 0.014 & 0.00114984 \\
\hline 11.41666667 & 11.53 & -0.12 & 0.014 & 0.001192914 \\
\hline 16.625 & 11.90 & 4.72 & 22.303 & 1.873833581 \\
\hline 6.75 & 11.47 & -4.72 & 22.303 & 1.944028451 \\
\hline df $=2$ & Chi Square at $0.025=7.378$ \\
\hline \multicolumn{5}{|c|}{$7.586>7.378$} \\
\hline
\end{tabular}




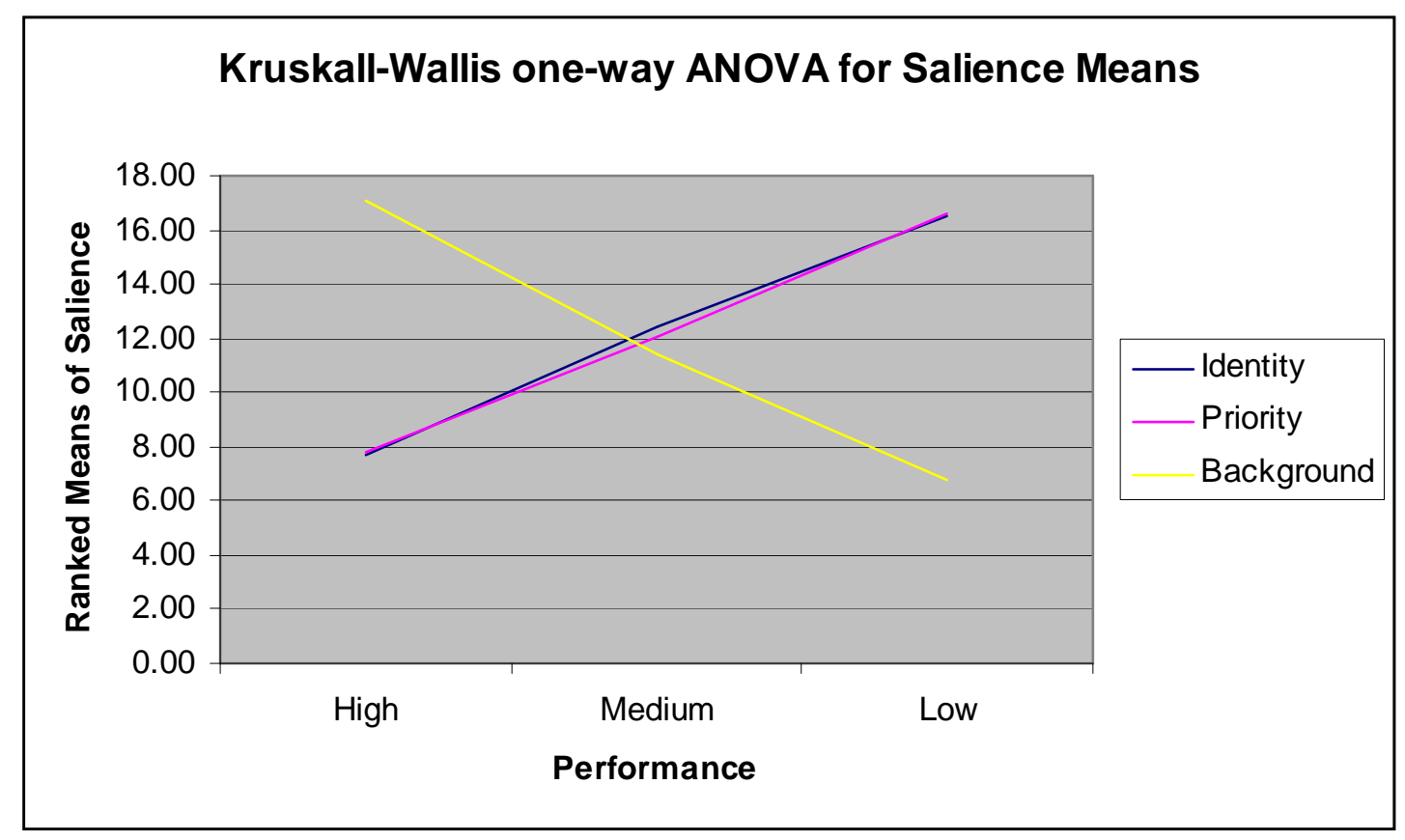

Figure 4: $H_{1}$ Hypothetical Case Results

The concluding results of this hypothetical test case is that evidence supports the hypothesis that High, Middle and Low performing organizations rank their common salience process types differently, and that Priority business processes measured correlate more positively to firm value than Background business processes (lower mean ranking level for high performers in Priority performance measures and lower mean ranking level for low performers in Background performance measurement) at a 0.05 significance level. It could not be demonstrated, however, that Identity business processes measured correlate more positively to firm value than Priority business processes at a 0.05 significance level. 
$\mathrm{H}_{2}$ : Nonfinancial performance measures are more correlated to firm value than financial measures as measured by Kruskal-Wallis one-way ANOVA.

Kruskal-Wallis one-way ANOVA is the alternative that will be used in this hypothesis test. To demonstrate the logic, two extreme examples will be used to show non-rejection of null then rejection of null. This will be followed by a hypothetical case using survey data measures.

To perform this test, the data is compiled and then ranked. Example 1 as follows in Table 21 where the number of measures are defined then ranked from $1-23$ in increasing order (tied numbers would be averaged). 
TABLE 21

KRUSKAL-WALLIS ONE-WAY ANOVA TEST RANKED DATA $\mathrm{H}_{2}$ EXAMPLE 1

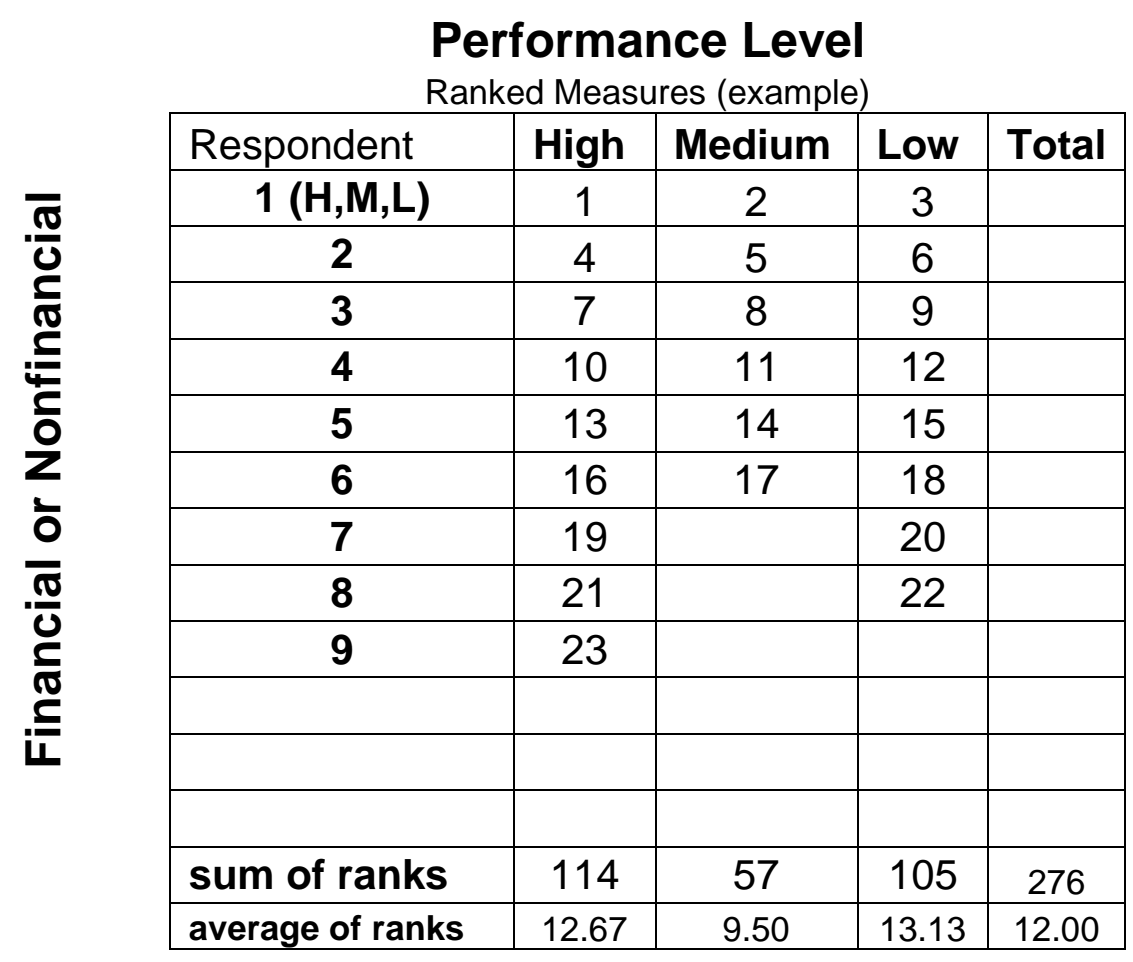

The Null Hypothesis cannot be rejected based on this sample data as exhibited in Table 22. 
TABLE 22

KRUSKAL-WALLIS TEST RESULTS $\mathrm{H}_{2}$ EXAMPLE 1

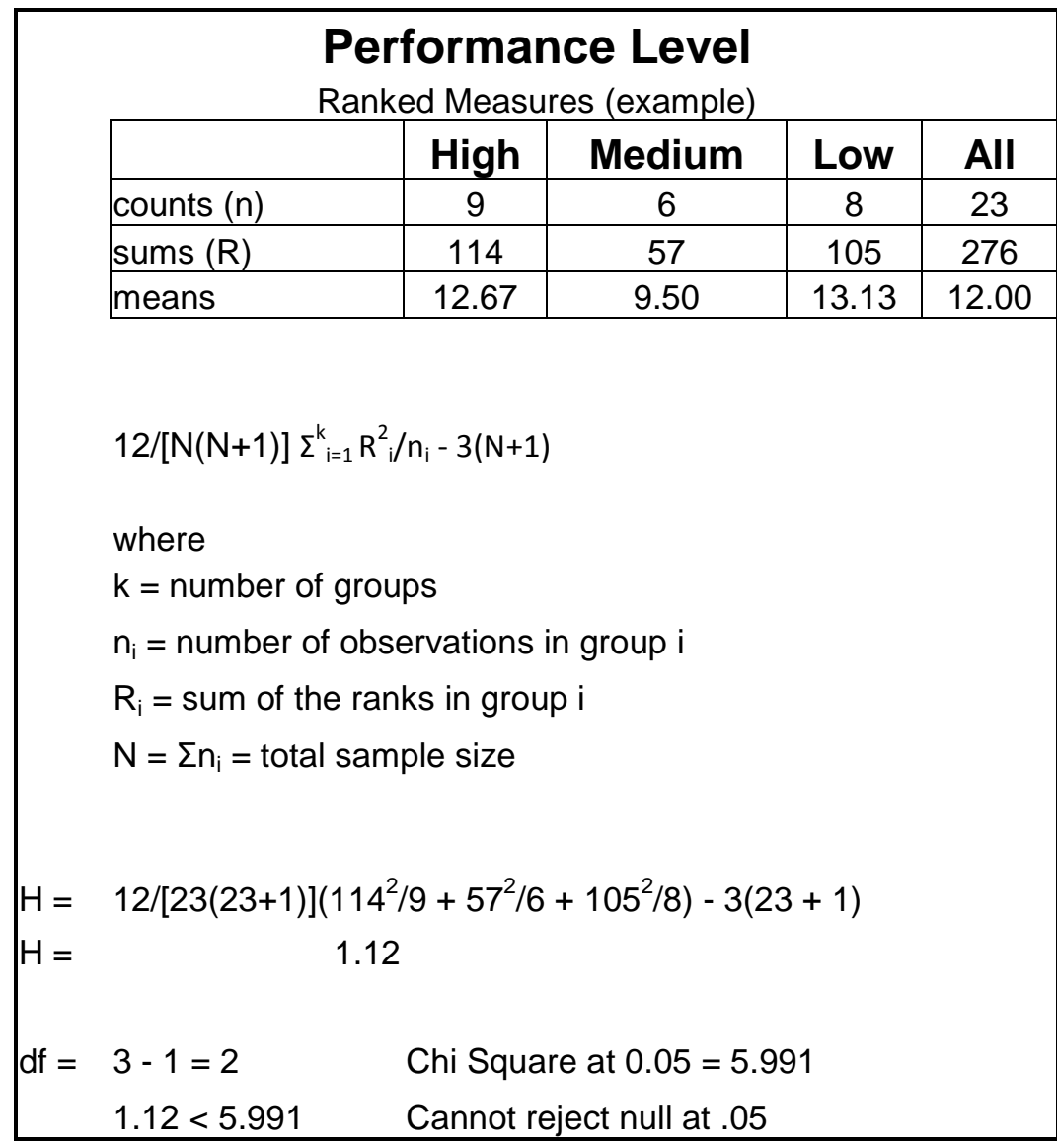

Table 23 gives an example (2) using the same process but with different responses where the null would be rejected at 0.05 . 
TABLE 23

KRUSKAL-WALLIS TEST RESULTS $\mathrm{H}_{2}$ EXAMPLE 2

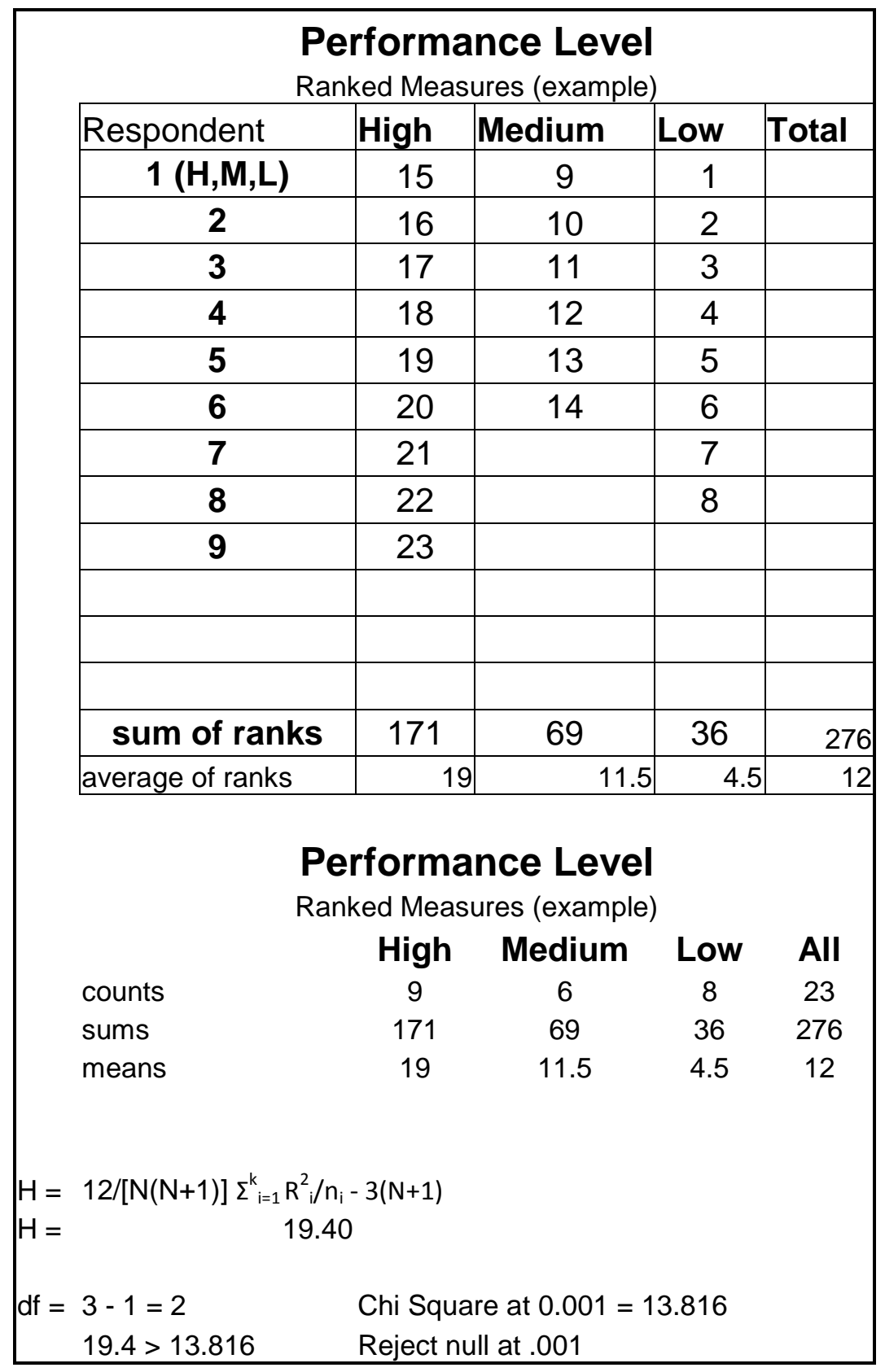

Having demonstrated an extreme example to show the logic of the test, the reasonableness of the test will be demonstrated using a hypothetical set of responses from 23 respondents as given in Table 24. 
TABLE 24

HYPOTHETICAL SURVEY RESPONSE FOR $\mathrm{H}_{2}$

\begin{tabular}{|c|c|c|c|c|}
\hline Respondent & $\begin{array}{c}\text { Performance } \\
\text { Level }\end{array}$ & Clockspeed & \multicolumn{2}{c|}{ Number of Measures Used } \\
\hline & & & Financial & Nonfinancial \\
\hline 1 & $\mathrm{H}$ & $\mathrm{H}$ & 2 & 5 \\
\hline 2 & $\mathrm{H}$ & $\mathrm{H}$ & 1 & 4 \\
\hline 3 & $\mathrm{H}$ & $\mathrm{H}$ & 3 & 6 \\
\hline 4 & $\mathrm{H}$ & $\mathrm{H}$ & 4 & 5 \\
\hline 5 & $\mathrm{H}$ & $\mathrm{H}$ & 2 & 3 \\
\hline 6 & $\mathrm{H}$ & $\mathrm{M}$ & 1 & 4 \\
\hline 7 & $\mathrm{H}$ & $\mathrm{M}$ & 5 & 3 \\
\hline 8 & $\mathrm{H}$ & $\mathrm{M}$ & 2 & 5 \\
\hline 9 & $\mathrm{H}$ & $\mathrm{L}$ & 3 & 2 \\
\hline 10 & $\mathrm{M}$ & $\mathrm{H}$ & 1 & 7 \\
\hline 11 & $\mathrm{M}$ & $\mathrm{H}$ & 5 & 2 \\
\hline 12 & $\mathrm{M}$ & $\mathrm{H}$ & 4 & 4 \\
\hline 13 & $\mathrm{M}$ & $\mathrm{M}$ & 3 & 3 \\
\hline 14 & $\mathrm{M}$ & $\mathrm{M}$ & 2 & 5 \\
\hline 15 & $\mathrm{M}$ & $\mathrm{L}$ & 1 & 1 \\
\hline 16 & $\mathrm{~L}$ & $\mathrm{H}$ & 5 & 1 \\
\hline 17 & $\mathrm{~L}$ & $\mathrm{H}$ & 4 & 2 \\
\hline 18 & $\mathrm{~L}$ & $\mathrm{H}$ & 3 & 3 \\
\hline 19 & $\mathrm{~L}$ & $\mathrm{H}$ & 3 & 4 \\
\hline 20 & $\mathrm{~L}$ & $\mathrm{M}$ & 2 & 2 \\
\hline 21 & $\mathrm{~L}$ & $\mathrm{M}$ & 4 & 4 \\
\hline 22 & $\mathrm{~L}$ & $\mathrm{~L}$ & 3 & 3 \\
\hline 23 & $\mathrm{~L}$ & $\mathrm{~L}$ & 1 & 1 \\
\hline & & & & \\
\hline
\end{tabular}

As in the example for $\mathrm{H}_{1}$, the raw responses are then organized in a matrix to rank all scores without regard to group membership in increasing rank based on the number of measures used from lowest to highest (like rates are "tied" and are therefore averaged) to determine a mean ranking for financial and nonfinancial as demonstrated in Table 25. 
TABLE 25

HYPOTHETICAL RESPONSES RANKING CONVERSIONS $\mathrm{H}_{2}$

\begin{tabular}{|c|c|c|c|c|c|}
\hline Financial & $\begin{array}{l}\text { Number of } \\
\text { Measures }\end{array}$ & $\begin{array}{c}\text { Tied - } \\
\text { Use Avg. }\end{array}$ & Nonfinancial & $\begin{array}{c}\text { Response } \\
\text { Count }\end{array}$ & $\begin{array}{c}\text { Tied - } \\
\text { Use Avg. }\end{array}$ \\
\hline 1 & 1 & & 1 & 1 & \\
\hline 1 & 2 & & 1 & 2 & \\
\hline 1 & 3 & & 1 & 3 & 2 \\
\hline 1 & 4 & & 2 & 4 & \\
\hline 1 & 5 & 3 & 2 & 5 & \\
\hline 2 & 6 & & 2 & 6 & \\
\hline 2 & 7 & & 2 & 7 & 5.5 \\
\hline 2 & 8 & & 3 & 8 & \\
\hline 2 & 9 & & 3 & 9 & \\
\hline 2 & 10 & 8 & 3 & 10 & \\
\hline 3 & 11 & & 3 & 11 & \\
\hline 3 & 12 & & 3 & 12 & 10 \\
\hline 3 & 13 & & 4 & 13 & \\
\hline 3 & 14 & & 4 & 14 & \\
\hline 3 & 15 & & 4 & 15 & \\
\hline 3 & 16 & 13.5 & 4 & 16 & \\
\hline 4 & 17 & & 4 & 17 & 15 \\
\hline 4 & 18 & & 5 & 18 & \\
\hline 4 & 19 & & 5 & 19 & \\
\hline 4 & 20 & 18.5 & 5 & 20 & \\
\hline 5 & 21 & & 5 & 21 & 19.5 \\
\hline 5 & 22 & & 6 & 22 & 22 \\
\hline 5 & 23 & 22 & 7 & 23 & 23 \\
\hline
\end{tabular}

The raw number of measures response data from Table 24 is then replaced with the rankings derived from Table 25 as summarized in Table 26. 
TABLE 26

RANKED SURVEY RESPONSE DATA $\mathrm{H}_{2}$

\begin{tabular}{|c|c|c|c|c|}
\hline \multirow[t]{2}{*}{ Respondent } & \multirow{2}{*}{$\begin{array}{c}\text { Performance } \\
\text { Level }\end{array}$} & \multirow{2}{*}{ Clockspeed } & \multicolumn{2}{|c|}{ Ranked Responses } \\
\hline & & & Financial & Nonfinancial \\
\hline 1 & $\mathrm{H}$ & $\mathrm{H}$ & 8 & 19.5 \\
\hline 2 & $\mathrm{H}$ & $\mathrm{H}$ & 3 & 15 \\
\hline 3 & $\mathrm{H}$ & $\mathrm{H}$ & 13.5 & 22 \\
\hline 4 & $\mathrm{H}$ & $\mathrm{H}$ & 18.5 & 19.5 \\
\hline 5 & $\mathrm{H}$ & $\mathrm{H}$ & 8 & 10 \\
\hline 6 & $\mathrm{H}$ & $\mathrm{M}$ & 3 & 15 \\
\hline 7 & $\mathrm{H}$ & $\mathrm{M}$ & 22 & 10 \\
\hline 8 & $\mathrm{H}$ & $M$ & 8 & 19.5 \\
\hline 9 & $\mathrm{H}$ & $\mathrm{L}$ & 13.5 & 5.5 \\
\hline 10 & M & $\mathrm{H}$ & 3 & 23 \\
\hline 11 & $\mathrm{M}$ & $\mathrm{H}$ & 22 & 5.5 \\
\hline 12 & $M$ & $\mathrm{H}$ & 18.5 & 15 \\
\hline 13 & $M$ & $M$ & 13.5 & 10 \\
\hline 14 & $M$ & $M$ & 8 & 19.5 \\
\hline 15 & $M$ & L & 3 & 2 \\
\hline 16 & $\mathrm{~L}$ & $\mathrm{H}$ & 22 & 2 \\
\hline 17 & $\mathrm{~L}$ & $\mathrm{H}$ & 18.5 & 5.5 \\
\hline 18 & $\mathrm{~L}$ & $\mathrm{H}$ & 13.5 & 10 \\
\hline 19 & $\mathrm{~L}$ & $\mathrm{H}$ & 13.5 & 15 \\
\hline 20 & $L$ & $M$ & 8 & 5.5 \\
\hline 21 & $\mathrm{~L}$ & $M$ & 18.5 & 15 \\
\hline 22 & $\mathrm{~L}$ & $\mathrm{~L}$ & 13.5 & 10 \\
\hline 23 & $L$ & $\mathrm{~L}$ & 3 & 2 \\
\hline
\end{tabular}

The ranked response means for High, Medium and Low performers for financial and nonfinancial measure use is then determined (Table 27) followed by the KruskalWallis one-way ANOVA test of the ranked means for each organization performance level in Table 28. 
TABLE 27

HIGH, MIDDLE AND LOW PERFORMER FIANCIAL AND NONFINANCIAL RANKED MEANS $\mathrm{H}_{2}$

\begin{tabular}{|c|c|c|c|c|}
\hline \multirow{2}{*}{ Respondent } & \multirow{2}{*}{ Level } & \multirow{2}{*}{ Clockspeed } & \multicolumn{2}{|c|}{ Ranked Responses } \\
\hline & & & Financial & Nonfinancial \\
\hline 1 & $\mathrm{H}$ & $\mathrm{H}$ & 8 & 19.5 \\
\hline 2 & $\mathrm{H}$ & $\mathrm{H}$ & 3 & 15 \\
\hline 3 & $\mathrm{H}$ & $\mathrm{H}$ & 13.5 & 22 \\
\hline 4 & $\mathrm{H}$ & $\mathrm{H}$ & 18.5 & 19.5 \\
\hline 5 & $\mathrm{H}$ & $\mathrm{H}$ & 8 & 10 \\
\hline 6 & $\mathrm{H}$ & $M$ & 3 & 15 \\
\hline 7 & $\mathrm{H}$ & $\mathrm{M}$ & 22 & 10 \\
\hline 8 & $\mathrm{H}$ & $\mathrm{M}$ & 8 & 19.5 \\
\hline 9 & $\mathrm{H}$ & $\mathrm{L}$ & 13.5 & 5.5 \\
\hline \multicolumn{3}{|c|}{ Count } & 9 & 9 \\
\hline \multicolumn{3}{|c|}{ sum of ranks } & 97.5 & 136.0 \\
\hline \multicolumn{3}{|c|}{ average of ranks } & 10.83 & 15.11 \\
\hline \multirow{2}{*}{ Respondent } & \multirow{2}{*}{ Level } & \multirow{2}{*}{ Clockspeed } & \multicolumn{2}{|c|}{ Ranked Responses } \\
\hline & & & Financial & Nonfinancial \\
\hline 1 & $M$ & $\mathrm{H}$ & 3 & 23 \\
\hline 2 & $M$ & $\mathrm{H}$ & 22 & 5.5 \\
\hline 3 & $M$ & $\mathrm{H}$ & 18.5 & 15 \\
\hline 4 & $\mathrm{M}$ & $\mathrm{M}$ & 13.5 & 10 \\
\hline 5 & $\mathrm{M}$ & $M$ & 8 & 19.5 \\
\hline 6 & $M$ & $\mathrm{~L}$ & 3 & 2 \\
\hline \multicolumn{3}{|c|}{ Count } & 6 & 6 \\
\hline \multicolumn{3}{|c|}{ sum of ranks } & 68.0 & 75.0 \\
\hline \multicolumn{3}{|c|}{ average of ranks } & 11.33 & 12.50 \\
\hline \multirow{2}{*}{ Respondent } & \multirow{2}{*}{ Level } & \multirow{2}{*}{ Clockspeed } & \multicolumn{2}{|c|}{ Ranked Responses } \\
\hline & & & Financial & Nonfinancial \\
\hline 1 & $\mathrm{~L}$ & $\mathrm{H}$ & 22 & 2 \\
\hline 2 & $\mathrm{~L}$ & $\mathrm{H}$ & 18.5 & 5.5 \\
\hline 3 & $\mathrm{~L}$ & $\mathrm{H}$ & 13.5 & 10 \\
\hline 4 & $\mathrm{~L}$ & $\mathrm{H}$ & 13.5 & 15 \\
\hline 5 & $\mathrm{~L}$ & $\mathrm{M}$ & 8 & 5.5 \\
\hline 6 & L & $M$ & 18.5 & 15 \\
\hline 7 & $\mathrm{~L}$ & $\mathrm{~L}$ & 13.5 & 10 \\
\hline 8 & $\mathrm{~L}$ & $\mathrm{~L}$ & 3 & 2 \\
\hline \multicolumn{3}{|c|}{ Count } & 8 & 8 \\
\hline \multicolumn{3}{|c|}{ sum of ranks } & 110.5 & 65.0 \\
\hline \multicolumn{3}{|c|}{ average of ranks } & 13.81 & 8.13 \\
\hline
\end{tabular}


TABLE 28

KRUSKAL-WALLIS TEST OF RANKED MEANS $\mathrm{H}_{2}$

\begin{tabular}{|c|c|c|c|c|}
\hline \multicolumn{5}{|c|}{$\begin{array}{c}\text { Performance Level } \\
\text { Ranked Number of Financial Measures }\end{array}$} \\
\hline & High & Medium & Low & All \\
\hline counts (n) & 9 & 6 & 8 & 23 \\
\hline sums $(R)$ & 97.5 & 68 & 110.5 & 276 \\
\hline means & 10.83 & 11.33 & 13.81 & 12.00 \\
\hline $\mathrm{H}=$ & \multicolumn{4}{|c|}{$\begin{array}{c}12 /[N(N+1)] \Sigma_{i=1}^{k} R_{i}^{2} / n_{i}-3(N+1) \\
0.90\end{array}$} \\
\hline $\mathrm{df}=$ & $\begin{array}{l}3-1=2 \\
0.90<5.991\end{array}$ & \multicolumn{3}{|c|}{$\begin{array}{l}\text { Chi Square at } 0.05=5.991 \\
\text { Cannot reject null at } .05\end{array}$} \\
\hline \multicolumn{5}{|c|}{$\begin{array}{c}\text { Performance Level } \\
\text { Ranked Number of Nonfinancial Measures }\end{array}$} \\
\hline & High & Medium & Low & All \\
\hline counts (n) & 9 & 6 & 8 & 23 \\
\hline sums $(\mathrm{R})$ & 136.0 & 75 & 65 & 276 \\
\hline means & 15.11 & 12.5 & 8.125 & 12.00 \\
\hline $\begin{array}{l}\mathrm{H}= \\
\mathrm{H}=\end{array}$ & \multicolumn{4}{|c|}{$\begin{array}{c}12 /[N(N+1)] \Sigma_{i=1}^{k} R_{i}^{2} / n_{i}-3(N+1) \\
4.54\end{array}$} \\
\hline $\mathrm{df}=$ & $\begin{array}{l}3-1=2 \\
4.54<5.991\end{array}$ & $\begin{array}{l}\text { Chi Square at } \\
\text { Cannot reject }\end{array}$ & $\begin{array}{l}=5.99 \\
\text { at } .05\end{array}$ & \\
\hline
\end{tabular}




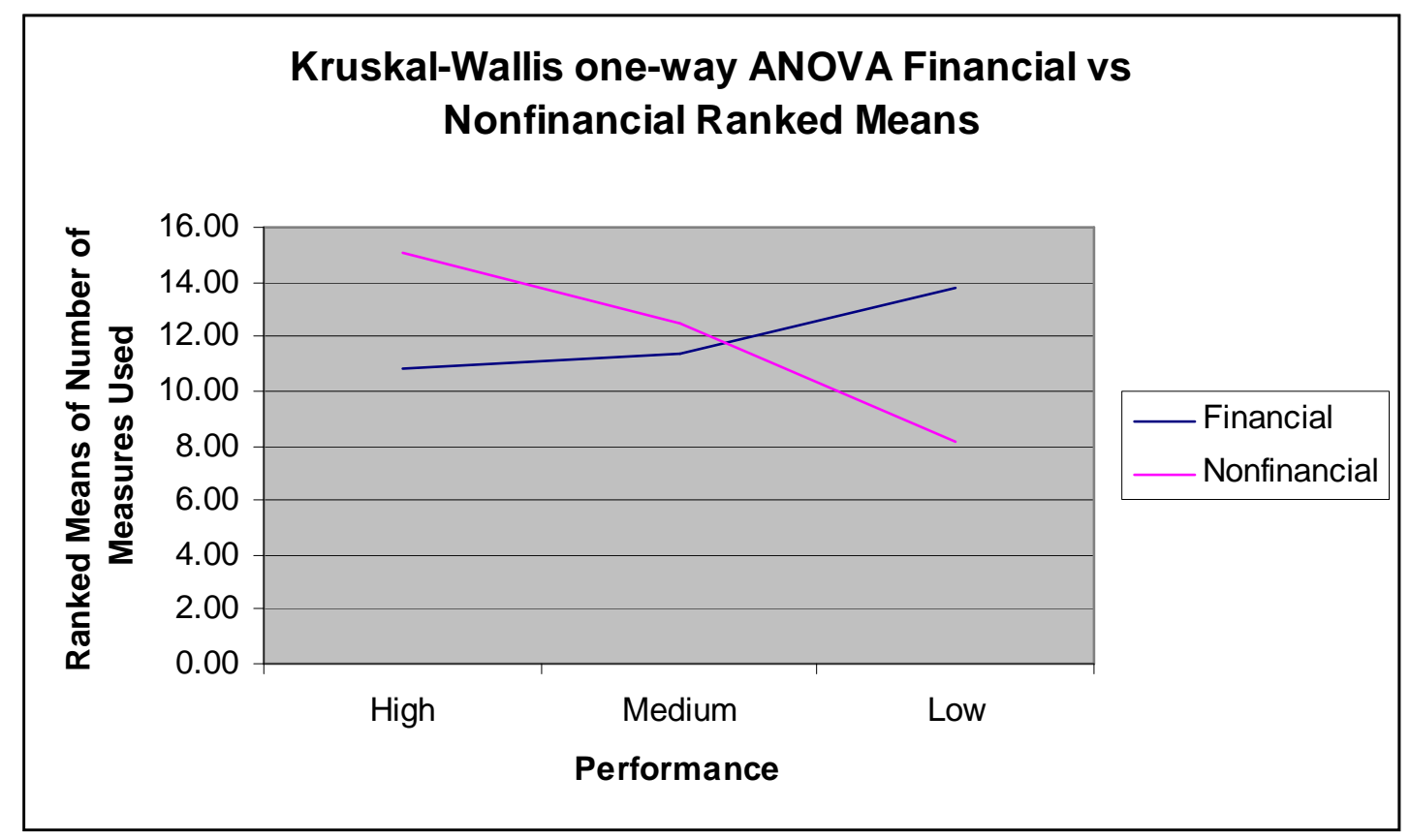

Figure 5: $\mathrm{H}_{2}$ Hypothetical Case Results

In this hypothetical case, financial measures appear to be used by high performers less than by low performers (lower ranking mean of 10.83 compared to 13.81 with middle performers in the middle ranking at 11.33), though statistical significance is not established. The nonfinancial measures appear to be used more by high performers (higher ranking mean of 15.11 compared to 8.13 with middle performers in the middle ranking at 12.5 ) though this was not statistically significant at 0.05 level. In conclusion, the hypothetical evidence appears to support the hypothesis that nonfinancial performance measures correlate more positively to firm value than financial measures, but not at a statistically significant level. 
$\mathrm{H}_{3}$ : The relative importance of nonfinancial performance measures compared to financial measures using the results from $\mathrm{H}_{2}$ is greater in high clock-speed industries than in low clock-speed industries as measured by Cramer's Phi.

The results from $\mathrm{H}_{2}$ (Financial / Nonfinancial) relative to three levels of clockspeed (high, medium, low) will be compared for strengths of association differences between the three-clockspeed categories. According to Howell (2002) "If I were going to retain only one measure of association, it would be Cramer's $\emptyset_{c} \ldots[$ which $]$ is not constrained by the size of the table...." (p. 165) and will therefore be used for this test.

For this test, the Chi Square value for each alternative needs to be determined since " $\ldots \varnothing_{\mathrm{c}}=\left(\chi^{2} /(\mathrm{N}(\mathrm{k}-1))\right)^{1 / 2}$ where $\mathrm{N}$ is the sample size and $\mathrm{k}$ is defined as the smaller of R and C" (Howell, 2002, p. 165).

The methodology will be demonstrated using the same hypothetical survey responses used to demonstrate $\mathrm{H}_{2}$ methodology in Table 24, and the ranked means in Table 26.

The first step is to break the data from the previous example into clockspeed tables to determine the ranking means for the variables as demonstrated in Tables $29-$ 31. 
TABLE 29

HIGH CLOCKSPEED FINANCIAL AND NONFINANCIAL RANKED MEANS $\mathrm{H}_{3}$

\begin{tabular}{|c|c|c|c|c|}
\hline \multicolumn{5}{|c|}{$\begin{array}{r}\text { Financial High Clockspeed } \\
\text { Performance }\end{array}$} \\
\hline Observed & High & Medium & Low & Total \\
\hline & 8 & 3 & 22 & \\
\hline & 3 & 22 & 18.5 & \\
\hline & 13.5 & 18.5 & 13.5 & \\
\hline & 18.5 & & 13.5 & \\
\hline & 8 & & & \\
\hline Count & 5 & 3 & 4 & 12 \\
\hline Mean & 10.20 & 14.50 & 16.88 & 41.58 \\
\hline \multicolumn{5}{|c|}{ Noninancial High Clockspeed } \\
\hline Observed & High & Medium & Low & Total \\
\hline & 19.5 & 23 & 2 & \\
\hline & 15 & 5.5 & 5.5 & \\
\hline & 22 & 15 & 10 & \\
\hline & 19.5 & & 15 & \\
\hline & 10 & & & \\
\hline Count & 5 & 3 & 4 & 12 \\
\hline Mean & 17.20 & 14.50 & 8.13 & 39.83 \\
\hline
\end{tabular}


TABLE 30

MEDIUM CLOCKSPEED FINANCIAL AND NONFINANCIAL RANKED MEANS $\mathrm{H}_{3}$

\begin{tabular}{|c|c|c|c|c|}
\hline \multicolumn{5}{|c|}{ Financial Medium Clockspeed } \\
\hline Observed & High & Medium & Low & Total \\
\hline & 3 & 13.5 & 8 & \\
\hline & 22 & 8 & 18.5 & \\
\hline & 8 & & & \\
\hline & & & & \\
\hline Count & 3 & 2 & 2 & \\
\hline Mean & 11.00 & 10.75 & 13.25 & 35.00 \\
\hline \multicolumn{5}{|c|}{$\begin{array}{c}\text { Nonfinancial Medium Clockspeed } \\
\text { Performance }\end{array}$} \\
\hline Observed & High & Medium & Low & Total \\
\hline & 15 & 10 & 5.5 & \\
\hline & 10 & 19.5 & 15 & \\
\hline & 19.5 & & & \\
\hline & & & & \\
\hline Count & 3 & 2 & 2 & \\
\hline Mean & 14.83 & 14.75 & 10.25 & \\
\hline
\end{tabular}


TABLE 31

LOW CLOCKSPEED FINANCIAL AND NONFINANCIAL RANKED MEANS $\mathrm{H}_{3}$

\begin{tabular}{|c|c|c|c|c|}
\hline \multicolumn{5}{|c|}{ Financial Low Clockspeed } \\
\hline \multicolumn{5}{|c|}{ Performance } \\
\hline Observed & High & Medium & Low & Total \\
\hline & 13.5 & 3 & 13.5 & \\
\hline & & & 3 & \\
\hline & & & & \\
\hline & & & & \\
\hline & & & & \\
\hline Count & 1 & 1 & 2 & 4 \\
\hline Mean & 13.50 & 3.00 & 8.25 & \\
\hline \multicolumn{5}{|c|}{ Noninancial Low Clockspeed } \\
\hline \multicolumn{5}{|c|}{ Performance } \\
\hline Observed & High & Medium & Low & Total \\
\hline & 5.5 & 2 & 10 & \\
\hline & & & 2 & \\
\hline & & & & \\
\hline & & & & \\
\hline & & & & \\
\hline Count & 1 & 1 & 2 & 4 \\
\hline Mean & 5.50 & 2.00 & 6.00 & \\
\hline
\end{tabular}

Chi-Squares are then calculated for the means for each clockspeed and performance level, followed by calculation of the Cramer's Phi as shown in Tables 32 34. 
TABLE 32

CRAMER'S PHI FOR HIGH CLOCKSPEED FINANCIAL VS. NONFINANCIAL RANKED USE MEANS $\mathrm{H}_{3}$

\begin{tabular}{|c|c|c|c|c|}
\hline & \multicolumn{4}{|c|}{$\begin{array}{l}\text { High Clockspeed } \\
\text { Performance }\end{array}$} \\
\hline Observed & High & Medium & Low & Total \\
\hline Financial & 10.20 & 14.50 & 16.88 & 41.58 \\
\hline Nonfinancial & 17.20 & 14.50 & 8.13 & 39.83 \\
\hline Total & 27.40 & 29.00 & 25.00 & 81.40 \\
\hline $\begin{array}{c}\text { Count } \\
\text { Expected }\end{array}$ & 10.00 & 6.00 & 8.00 & 24.00 \\
\hline Financial & 13.99 & 14.81 & 12.77 & \\
\hline Nonfinancial & 13.41 & 14.19 & 12.23 & \\
\hline Observed & Expected & $O-E$ & $\begin{array}{l}\mathrm{(O-} \\
\mathrm{E})^{\wedge} 2\end{array}$ & $(\mathrm{O}-\mathrm{E})^{\wedge} 2 / \mathrm{E}$ \\
\hline 10.20 & 13.99 & -3.79 & 14.398 & 1.028865 \\
\hline 17.20 & 13.41 & 3.79 & 14.398 & 1.074075 \\
\hline 14.50 & 14.81 & -0.31 & 0.097 & 0.006561 \\
\hline 14.50 & 14.19 & 0.31 & 0.097 & 0.006849 \\
\hline 16.875 & 12.77 & 4.11 & 16.861 & 1.320524 \\
\hline 8.125 & 12.23 & -4.11 & 16.861 & 1.37855 \\
\hline $\mathrm{df}=2$ & Chi Square & $0.05=5.99$ & & 4.815424 \\
\hline $\begin{array}{l}\phi_{\mathrm{c}}=\left(\chi^{2} /(\mathrm{N}(\mathrm{k}-1))\right. \\
\phi_{\mathrm{c}}=\end{array}$ & 0.44793154 & where & $\begin{array}{l}\mathrm{N}=\text { San } \\
\mathrm{k}=\mathrm{sma} \\
\text { and C }\end{array}$ & $\begin{array}{l}\text { ple size } \\
\text { er of } R\end{array}$ \\
\hline
\end{tabular}


TABLE 33

CRAMER'S PHI FOR MEDIUM CLOCKSPEED FINANCIAL VS. NONFINANCIAL RANKED USE MEANS $\mathrm{H}_{3}$

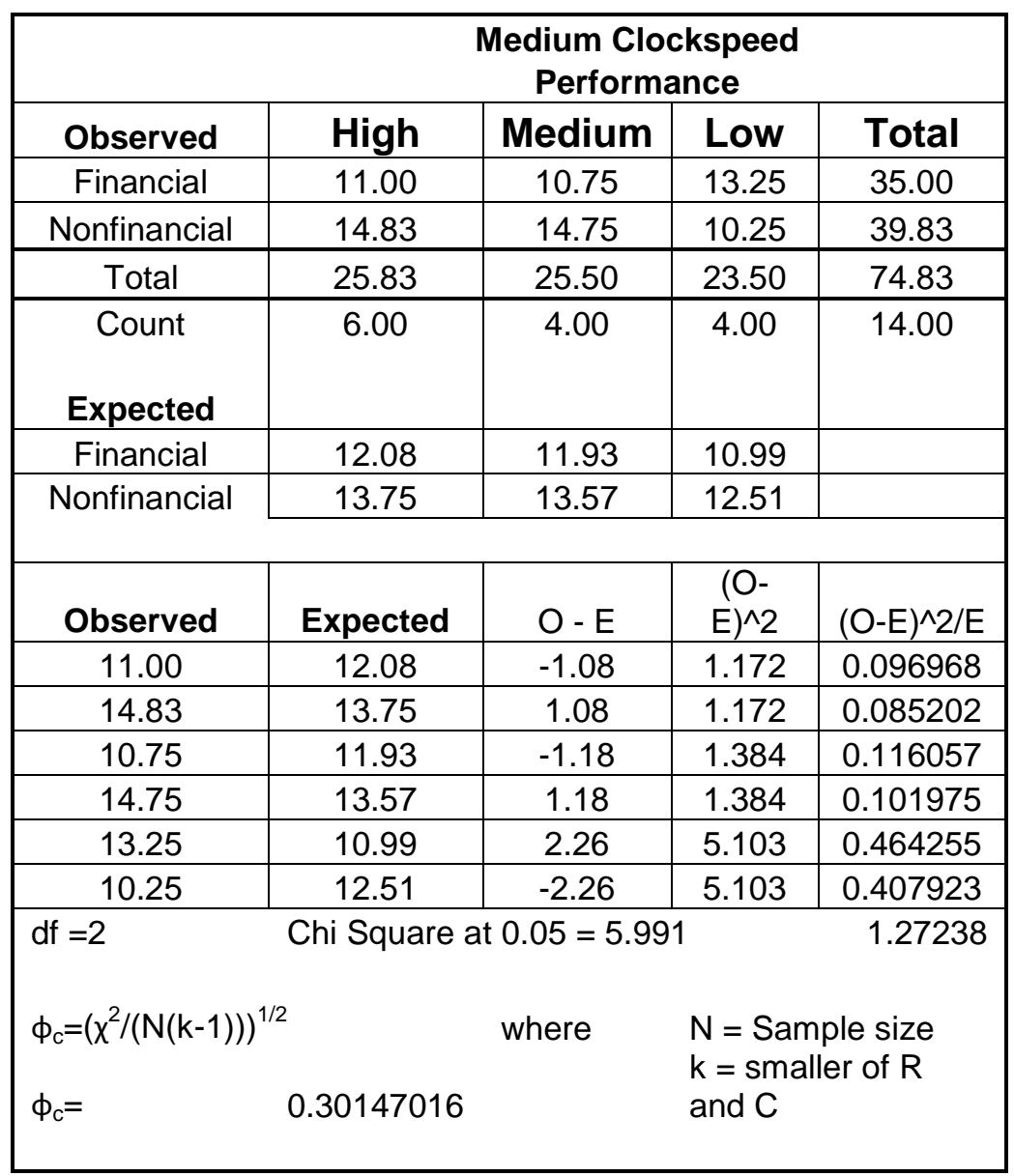


TABLE 34

CRAMER'S PHI FOR LOW CLOCKSPEED FINANCIAL VS. NONFINANCIAL RANKED USE MEANS $\mathrm{H}_{3}$

\begin{tabular}{|c|c|c|c|c|}
\hline \multicolumn{5}{|c|}{$\begin{array}{l}\text { Low Clockspeed } \\
\text { Performance }\end{array}$} \\
\hline Observed & High & Medium & Low & Total \\
\hline Financial & 13.50 & 3.00 & 8.25 & 24.75 \\
\hline Nonfinancial & 5.50 & 2.00 & 6.00 & 13.50 \\
\hline Total & 19.00 & 5.00 & 14.25 & 38.25 \\
\hline $\begin{array}{c}\text { Count } \\
\text { Expected }\end{array}$ & 2.00 & 2.00 & 4.00 & 8.00 \\
\hline Financial & 12.29 & 3.24 & 9.22 & \\
\hline Nonfinancial & 6.71 & 1.76 & 5.03 & \\
\hline Observed & Expected & $O-E$ & $\begin{array}{l}(\mathrm{O}- \\
\mathrm{E})^{\wedge} 2\end{array}$ & $(O-E)^{\wedge} 2 / E$ \\
\hline 13.50 & 12.29 & 1.21 & 1.454 & 0.11828 \\
\hline 5.50 & 6.71 & -1.21 & 1.454 & 0.216847 \\
\hline 3.00 & 3.24 & -0.24 & 0.055 & 0.017112 \\
\hline 2.00 & 1.76 & 0.24 & 0.055 & 0.031373 \\
\hline 8.25 & 9.22 & -0.97 & 0.942 & 0.102167 \\
\hline 6 & 5.03 & 0.97 & 0.942 & 0.187307 \\
\hline $\mathrm{df}=2$ & Chi Square & $0.05=5.99$ & & 0.673086 \\
\hline $\begin{array}{l}\phi_{c}=\left(\chi^{2} /(N(\mathrm{k}-1))\right) \\
\phi_{c}=\end{array}$ & 0.29006166 & where & $\begin{array}{l}N=\text { Sar } \\
\mathrm{k}=\mathrm{sma} \\
\text { and } \mathrm{C}\end{array}$ & $\begin{array}{l}\text { le size } \\
\text { ef of } R\end{array}$ \\
\hline
\end{tabular}




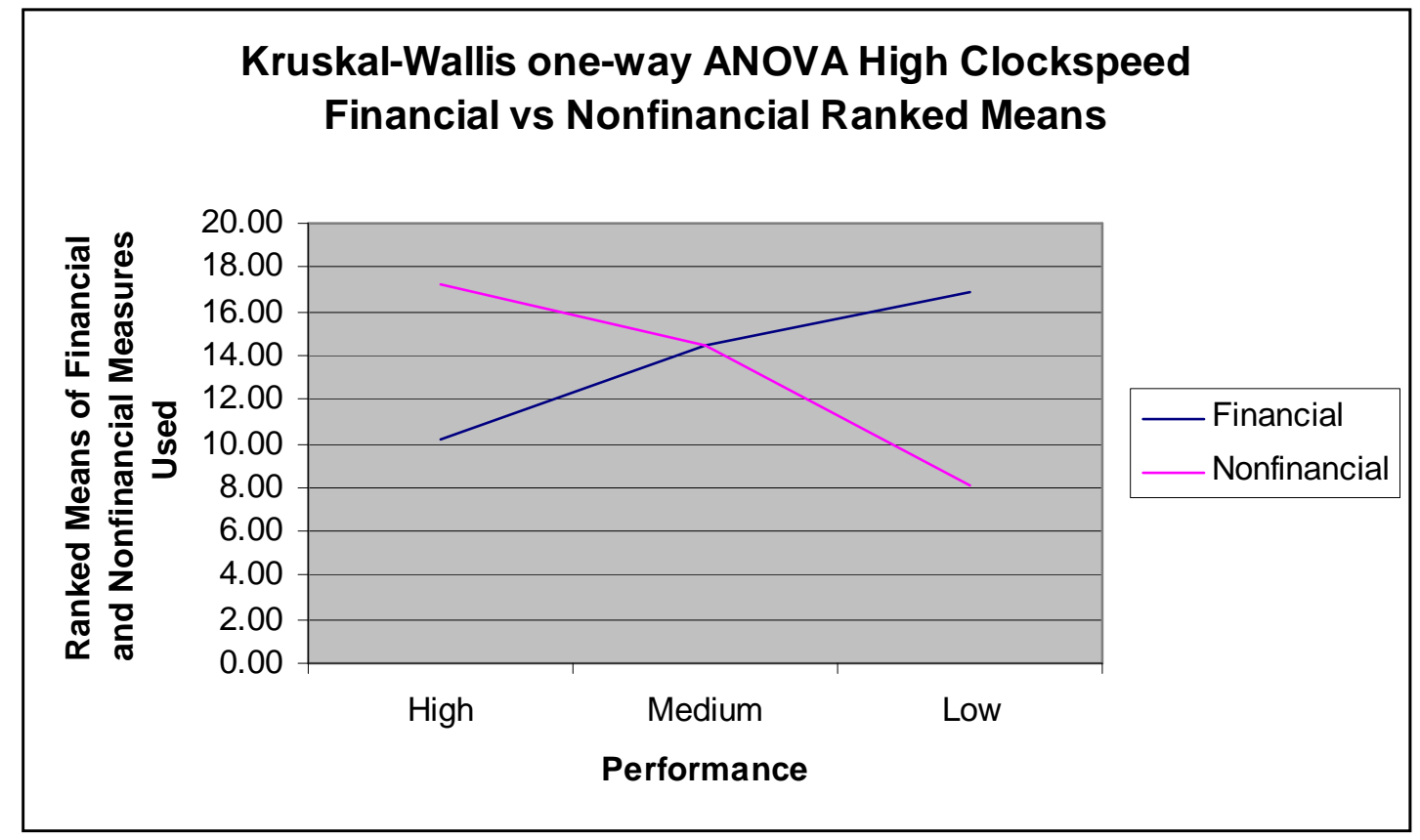

Figure 6: $\mathrm{H}_{3}$ High Clockspeed Hypothetical Case Results

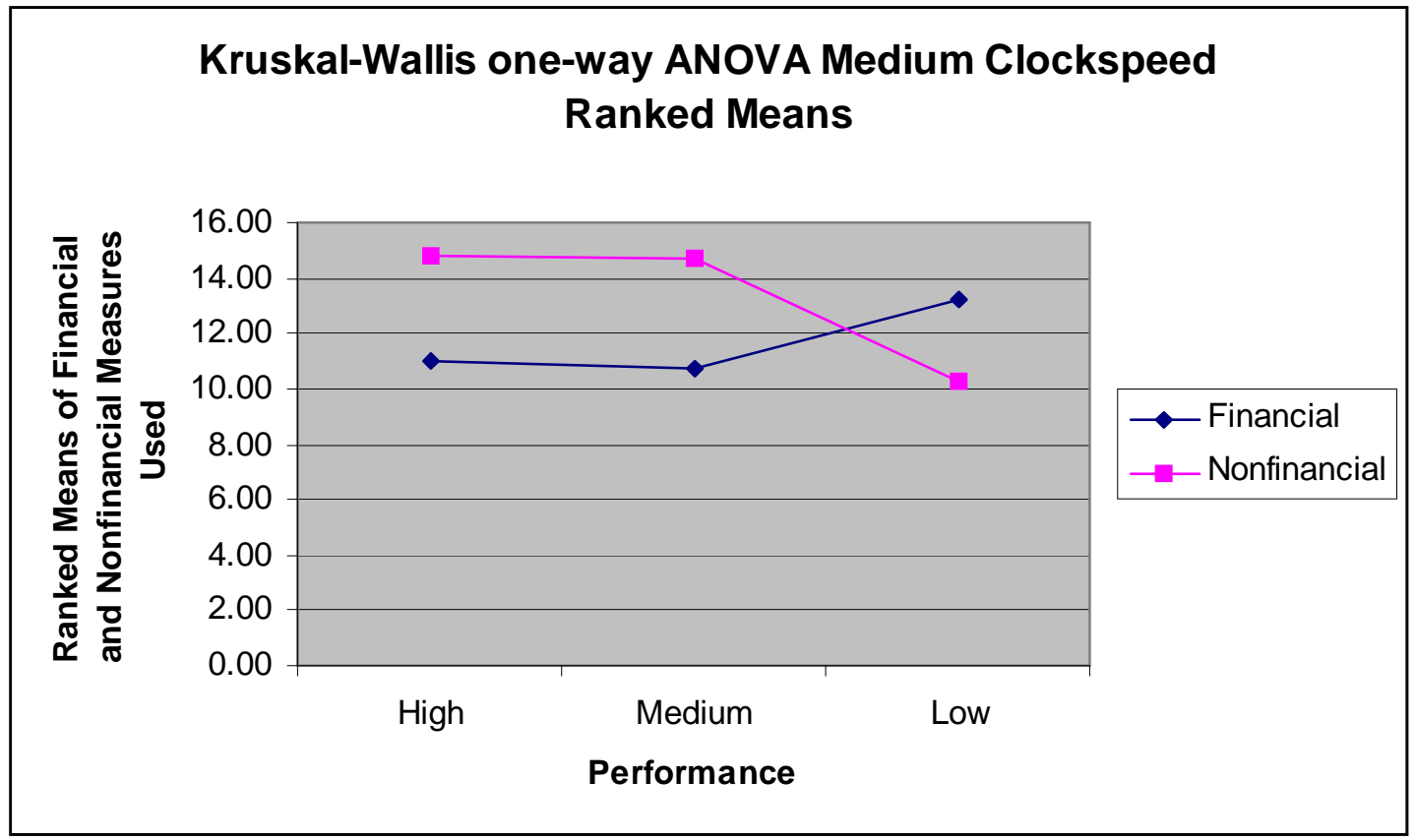

Figure 7: $\mathbf{H}_{3}$ Medium Clockspeed Hypothetical Case Results 


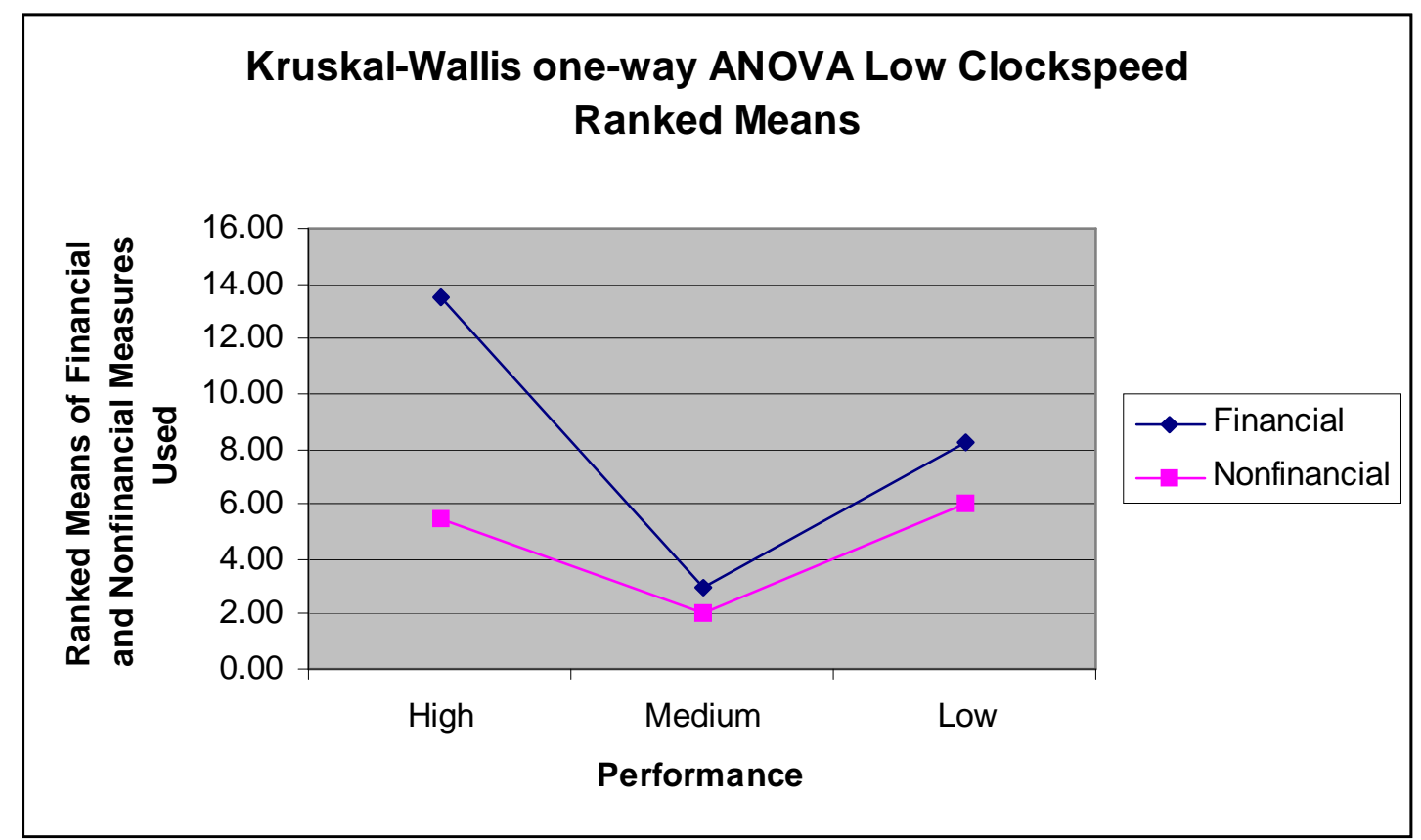

Figure 8: $\mathrm{H}_{3}$ Low Clockspeed Hypothetical Case Results

The results of this hypothetical test gives evidence to support the hypothesis that the relative importance of nonfinancial performance measures compared to financial measures is greater in high clockspeed industries than in low clock-speed industries. The degree of association for High Clockspeed industries is 0.45 compared to 0.30 for Medium and 0.29 for Low Clockspeed industries.

$\mathrm{H}_{4}$ : The number of types of Balanced Scorecard metrics used correlate to firm value as measured by Kruskal-Wallis one-way ANOVA.

Kruskal-Wallis one-way ANOVA is the alternative that will be used with the same methodology as described in Tables 10 - 13 for Hypothesis 1. 


\section{Conclusions}

Viable testing methods are defined for each of the hypotheses under consideration and examples of their use provided. In all cases nonparametric tests are necessary. It is anticipated that the questionnaire will yield a much higher number of responses than are used in the hypothetical cases. The questionnaire is designed to yield a high response rate by limiting the number of questions to 10 , thereby requiring minimal time of respondents. Once the infrastructure is set up (database of contact email addresses and web based survey), a large volume of requests for response can be sent to a broad range of companies for little cost.

Following the test, Chapter 5 documents the results, and Chapter 6 discusses the results and provides recommendations for further research. 


\section{CHAPTER V}

\section{RESULTS}

Implementation of Methods and Techniques as proposed in Chapter IV with subsequent results are documented in this chapter in the following sequence: 1) Sample Definition and Survey Setup; 2) Pilot Test Observations / Conclusions / Plan; 3) Dissertation Survey Results; and finally 4) Hypothesis Testing relative to each hypothesis under consideration. Chapter VI concludes this research with a discussion of: results and implications of the research; its limitations and strengths; then provides suggestions for further research.

\section{Sample Definition and Survey Setup}

The first step taken in sample definition and setup is to identify and define NAICS Categories to standardize performance among industry types. A total of 19,720 six-digit NAICS codes for a total of 13,286 companies are identified using Mergent Online, of which 7,268 companies are later determined to be active. This resolution is deemed too high to be of use so the number of codes, and the number of companies for each code, are determined based on 2-Digit, 3-Digit, and 4-Digit Code Classification Schemes (Appendix H). Results of the search are summarized as follows in Table 35: 
TABLE 35

NUMBER OF COMPANIES PER NAICS CATEGORY SUMMARY

\begin{tabular}{|l|r|r|r|}
\hline Total 6-Digit NAICS Codes $\quad \mathbf{1 9 , 7 2 0}$ & \multicolumn{1}{l|l|}{$\begin{array}{l}\text { 2-Digit } \\
\text { Code }\end{array}$} & $\begin{array}{l}\text { 3-Digit } \\
\text { Code }\end{array}$ & \multicolumn{1}{l|}{$\begin{array}{l}\text { 4-Digit } \\
\text { Code }\end{array}$} \\
\hline Total (Number of Companies) & 13,286 & 13,286 & 13,286 \\
\hline Max (Companies / Category) & 3,573 & 1,628 & 1,484 \\
\hline Min (Companies / Category) & 15 & 1 & 1 \\
\hline Count (NAICS Categories) & 24 & 87 & 289 \\
\hline Average (Companies / Category) & 554 & 153 & 46 \\
\hline Standard Deviation (Companies / Category) & 834.53 & 282.22 & 134.13 \\
\hline & & & \\
\hline
\end{tabular}

A 3-Digit Code Classification Scheme is used since it has greater resolution than a 2-Digit Scheme (87 total categories compared to 24 categories) with substantially fewer categories than a 4-Digit classification scheme (87 total categories compared to 289 categories) while the maximum number of companies for any one category is comparable to that of the 4-Digit Scheme (1,628 companies compared to 1,484 companies). The 3Digit Code Classification Scheme is then used to download all companies from Mergent Online. A systematic selection process is defined based on a 5\% sampling ratio, which is subsequently increased to a $10 \%$ sampling ratio for the pilot test (Appendix I). The first date with stock price history is then found for each sample company from Yahoo Finance. By converting the date to years publicly traded as of $12 / 31 / 08$ a table is created with the number of companies at $3,5,10,15,20,25,30$ years. LexisNexis is used to find general email addresses for companies that are publicly traded $\geq 5$ years and to the extent possible for executives. For those not available Hoovers Online is used to augment the contact data. Response rate is anticipated to be $2 \%-5 \%$. The summary of results follows in Table 36: 
TABLE 36

PILOT TEST SAMPLE SPECIFICATION

\begin{tabular}{|l|r|r|r|r|r|r|r|r|r|}
\hline & Total & $\begin{array}{l}\text { 3 } \\
\text { yrs }\end{array}$ & $\begin{array}{l}\mathbf{5} \\
\text { yrs }\end{array}$ & $\begin{array}{l}\mathbf{1 0} \\
\text { yrs }\end{array}$ & $\begin{array}{l}\mathbf{1 5} \\
\text { yrs }\end{array}$ & $\begin{array}{l}\mathbf{2 0} \\
\text { yrs }\end{array}$ & $\begin{array}{l}\mathbf{2 5} \\
\text { yrs }\end{array}$ & $\begin{array}{l}\mathbf{3 0} \\
\text { yrs }\end{array}$ & $\begin{array}{l}\text { 5yrs } \\
\text { w/email }\end{array}$ \\
\hline Set 1 (5\%) \# of Companies & 349 & 267 & 230 & 161 & 99 & 40 & 6 & 2 & 183 \\
\hline Percent of Total & & $77 \%$ & $66 \%$ & $46 \%$ & $28 \%$ & $11 \%$ & $2 \%$ & $1 \%$ & $52 \%$ \\
\hline Set 2 (5\%) \# of Companies & 352 & 257 & 222 & 152 & 89 & 27 & 5 & 2 & 177 \\
\hline Percent of Total & & $73 \%$ & $63 \%$ & $43 \%$ & $25 \%$ & $8 \%$ & $1 \%$ & $1 \%$ & $50 \%$ \\
\hline Combined (10\% Sample) & $\mathbf{7 0 1}$ & $\mathbf{5 2 4}$ & $\mathbf{4 5 2}$ & $\mathbf{3 1 3}$ & $\mathbf{1 8 8}$ & $\mathbf{6 7}$ & $\mathbf{1 1}$ & $\mathbf{4}$ & $\mathbf{3 6 0}$ \\
\hline Percent of Total & & $\mathbf{7 5 \%}$ & $\mathbf{6 4 \%}$ & $\mathbf{4 5 \%}$ & $\mathbf{2 7 \%}$ & $\mathbf{1 0} \%$ & $\mathbf{2} \%$ & $\mathbf{1 \%}$ & $\mathbf{5 1 \%}$ \\
\hline
\end{tabular}

The Survey Methodology is then defined based on Mail and Internet Surveys: The

Tailored Design Method (2nd ed.) (Dillman, 2000, pp. 156 - 185) as follows (Appendix $\mathrm{J})$ :

- Week 1 -- $(02 / 24 / 09)$ Tue 1 st Contact Email Prior Letter

- Week 2 -- $(02 / 27 / 09) \mathrm{Fr}$ 2nd Contact Survey Cover Letter

- Week 3 -- (03/09/09) Mon 3rd Contact E-mail Thank You/Reminder

- Week 5 -- (03/23/09) Mon 4th Contact Repeat Questionnaire

- Week 10 -- (04/27/09) Mon 5th Final Contact

WebSurveyor by Vovici (available through Portland State University OIT) is used as the tool for survey distribution.

\section{Pilot Test Observations / Conclusions / Plan}

Six observations and four comments from the Pilot Test are used to define the plan for final Dissertation Survey implementation as follows: 


\section{Observation I:}

TABLE 37

PILOT TEST COMPANIES AND COMPLETION RATE

\begin{tabular}{lrr} 
& \multicolumn{1}{c}{ Total } & \multicolumn{1}{l}{ Percent } \\
$10 \%$ Sample & 701 & $100.00 \%$ \\
5 Years Plus & 452 & $64.48 \%$ \\
W/ email info & 360 & $51.36 \%$
\end{tabular}

\begin{tabular}{lrrr} 
Test & Contacts & Unreachable & $\begin{array}{l}\text { Survey } \\
\text { Completion }\end{array}$ \\
\hline Pilot Test 1 & 360 & 4 & $\mathbf{7}$ \\
Pilot Test 2 & 352 & & $\mathbf{1}$ \\
Pilot Test 3 & 344 & & $\mathbf{5}$ \\
Pilot Test 4 & 332 & 1 & $\mathbf{3}$ \\
Pilot Test 5 & 324 & & $\mathbf{1 6}$ \\
\hline Total & & 5 & $\mathbf{4 . 4 4 \%}$
\end{tabular}

Conclusions: Completion rate is within the initial estimate of expected responses of $2 \%-5 \%$. Given that solicitation is restricted to a generic email address for each company with an arbitrarily selected officer of the company as the noted contact the response rate is acceptable. A substantial loss of potential respondents is realized due to the lack of email address availability (452 companies publicly traded $\geq 5$ years reduced to 360 companies publicly traded $\geq 5$ years with email address $=20 \%$ loss of potential contacts). 


\section{Observation II:}

NAICS Category Pilot Test Completions in order of completion:

TABLE 38

PILOT TEST NAICS CATEGORY COMPLETIONS

\begin{tabular}{|rcl|}
\hline \multicolumn{1}{l}{$\begin{array}{l}\text { Date \& Time } \\
\text { Submitted }\end{array}$} & $\begin{array}{l}\text { NAICS } \\
\text { Code }\end{array}$ & Description \\
\hline 2/27/2009 11:11 & 325 & Chemical Manufacturing \\
2/27/2009 11:56 & 336 & Transportation Equipment Manufacturing \\
2/27/2009 12:06 & 423 & Merchant Wholesalers, Durable Goods \\
2/27/2009 12:52 & 722 & Food Services and Drinking Places \\
3/2/2009 8:14 & 423 & Merchant Wholesalers, Durable Goods \\
3/2/2009 14:46 & 525 & Funds, Trusts, and Other Financial Vehicles \\
3/6/2009 8:51 & 713 & Amusement, Gambling, \& Recreation Industries \\
$3 / 9 / 200911: 19$ & 812 & Personal and Laundry Services \\
3/24/2009 6:42 & 541 & Professional, Scientific, and Technical Services \\
$3 / 24 / 200911: 27$ & 541 & Professional, Scientific, and Technical Services \\
$3 / 26 / 200915: 47$ & 325 & Chemical Manufacturing \\
4/1/2009 15:30 & 221 & Utilities \\
4/3/2009 6:48 & 237 & Heavy and Civil Engineering Construction \\
4/27/2009 17:25 & 311 & Food Manufacturing \\
4/28/2009 10:56 & 325 & Chemical Manufacturing \\
$4 / 29 / 200914: 26$ & 311 & Food Manufacturing \\
\hline
\end{tabular}

Conclusion: Participation is well represented across industries indicating that the survey is applicable across the full range of NAICS Categories. 


\section{Observation III:}

Finance Related Title/Department Solicitations vs. Finance Related Title/Department Responses:

- Finance Related Solicitations:

- Finance Related Rejections:

- Finance Related Completions:
$7.25 \%$ of all solicitations

$7.69 \%$ (Company Policy) of all rejections

$31.25 \%$ of all completions

Conclusion: A very high percentage of survey completions are from finance related title/department staff relative to the number of finance related title/department solicitations. The total percentage of finance related rejections are comparable to the percentage of finance related solicitations. Therefore -- Target Finance Related Contacts for solicitation.

\section{Observation IV:}

Several companies indicated that the survey would be forwarded to the appropriate department or person. Multiple responses from each company did not occur with the exception of one case; while statements were received from some companies that the individual noted on the cover letter does not respond to surveys.

Conclusion: Target finance related contacts when possible and delete the request for multiple responses from each company. As an additional benefit, this may result in better consistency of responses and may eliminate the need to introduce some type of averaging mechanism to address the multiple response scenarios. 
Observation V:

TABLE 39

\section{PILOT TEST FINANCIAL - NONFINANCIAL MEASURES}

\begin{tabular}{|l|rrrr|}
\hline & \multicolumn{4}{|c|}{ Number of Measures Used } \\
Respondent & Financial & Operating & Employee & Customer \\
\hline Finance & 10 & 5 & 5 & 3 \\
Finance & 4 & 7 & 2 & 5 \\
Finance & 50 & 20 & 15 & 20 \\
Corporate & 20 & 30 & 5 & 25 \\
Finance & 10 & 12 & 6 & 10 \\
Finance & 4 & 20 & 2 & 20 \\
Corporate & 5 & 20 & 5 & 5 \\
Corporate & 20 & 20 & 5 & 10 \\
Corporate & 6 & 8 & 10 & 8 \\
Corporate & 20 & 30 & 15 & 20 \\
Corporate & 20 & 100 & 20 & 50 \\
Corporate & 10 & 3 & 4 & 3 \\
HR & 10 & 10 & 5 & 8 \\
Corporate & 14 & 15 & 0 & 3 \\
Corporate & 9 & 8 & 4 & 3 \\
\hline Max & 50 & 100 & 20 & 50 \\
Min & 4 & 3 & 0 & 3 \\
Average & 14.13 & 20.53 & 6.87 & 12.87 \\
\hline Excluded first respondent as relative non- respondent all 0 \\
\hline \multicolumn{5}{r}{}
\end{tabular}

Conclusion: Good variation in ranking between categories. 


\section{Observation VI:}

TABLE 40

\section{PILOT TEST PROCESS SALIENCE RANKING RESPONSES}

\begin{tabular}{|c|c|c|c|c|}
\hline Respondent & Rank 1 & Rank 2 & Rank 3 & Rank 4 \\
\hline Finance & Identity & Background & Mandated & Priority \\
\hline Finance & Priority & Background & Identity & Mandated \\
\hline Finance & Identity & Priority & Background & Mandated \\
\hline Corporate & Mandated & Background & Identity & Priority \\
\hline Finance & Priority & Background & Identity & Mandated \\
\hline Finance & Identity & Background & Priority & Mandated \\
\hline Corporate & Priority & Mandated & Background & Identity \\
\hline Corporate & Identity & Priority & Background & Mandated \\
\hline Corporate & Priority & Mandated & Background & Identity \\
\hline Corporate & Background & Mandated & Priority & Identity \\
\hline Corporate & Identity & Priority & Background & Mandated \\
\hline Corporate & Background & Priority & Mandated & Identity \\
\hline & Background & Mandated & Priority & Identity \\
\hline Corporate & Background & Priority & Identity & Mandated \\
\hline Corporate & Background & Mandated & Identity & Priority \\
\hline Identity & 5 & 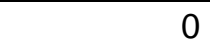 & 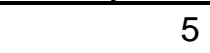 & 5 \\
\hline Priority & 4 & 5 & & 3 \\
\hline Background & 5 & 5 & 5 & 0 \\
\hline Mandated & 1 & 5 & 2 & 7 \\
\hline
\end{tabular}

Conclusions: There is good variability of response. The quality of question receives no written comments or questions, indicating that respondents had no problem with the survey which is therefore considered valid in its format. 


\section{Survey Comment I:}

"We are a very small biotechnology R\&D company that outsources most of our operational tasks. Most of your performance questions do not pertain to our current business model."

Conclusion: This was first survey respondent whose only response was "don’t know."

Solicitation was to CEO; President; Director, and completion noted Executive, Corporate, therefore target finance related contacts.

\section{Survey Comment II:.}

"With all the junk mail going around it took me a while to verify that this request was valid. I found some phone numbers after I clicked on the link but clicking on links from unknown sources in emails is not considered safe." [Note: This comment on survey from Pilot Test 5]

Conclusion: Identify HSRRC Proposal \# and HSRRC phone number on each cover letter so potential respondents can easily confirm validity of survey.

\section{Survey Comment III:.}

"The \# of estimated measures relates to the entire corporation. Of just the "Key" metrics; there are about 150-200 in total across Fin'l; Cust; Oper \& Employee dimensions with many other "tracking metrics" to keep tabs on more detailed breakdowns of performance. (On Q8; I tried entering 150 but was limited to 100 by the survey). If you have further questions call [deleted for anonymity]. I would like to receive the overall results of your survey at [deleted for anonymity]. Thanks."

Conclusion: Increase maximum number available for entry to 999. 


\section{Survey Non-Respondent Email Comment IV:}

"Dear Robert,

Being in the financial service industry especially during these economic times, it's necessary for our CEO to focus primarily on the business of increasing our stock price for our stockholders. Unfortunately, we do not have the luxury -- as we did several years ago -- to take time out of our day to complete a survey. My apologies, but unfortunately, the priority is not there right now."

Conclusion: Financial Industry turmoil at the time of the Pilot Test is

documented. Once again, perhaps targeting financial contact instead of CEO or Chairman may yield better completion rate. Include the estimated $3-5$ minutes in addition to the number of questions to highlight low time requirement on all solicitations.

\section{Pilot Test Results Conclusions:}

Pilot Test Results Conclusions are as follows. The Pilot Test is successful. Respondents understand the questions and no major flaws are detected in the study. Therefore the survey instrument can be used as in the Pilot Test. In addition the results can be combined for analysis. However, minor variations will be made in the Final Test including: 1) Note of “...brief 3 - 5 minute, 12 question questionnaire...” on all solicitation cover letters instead of the “...brief 12 question questionnaire..." comment used in the pilot test cover letters; 2) The contact noted on the email to generic address will be targeted to $\mathrm{CFO} /$ Accounting or some other finance related function when possible; 3) The comment regarding structure allowing multiple responses from one company will be deleted; 4) Identify HSRRC phone number and application number on every cover letter to help potential respondents confirm validity of the study. 


\section{Changes for Testing Based on Pilot Test:}

The plan following the Pilot test was stated as follows: Prepare Final Test based on $50 \%$ sample rate (expected $\mathrm{N}$ approximately 80) using the Systematic Selection Process used in the Pilot Test. The Pilot Test selected every $10^{\text {th }}$ company based on a random start number from 1 to 10 for each three digit NAICS category. The final selection will include every odd or even numbered company depending on the original random start number for the Pilot Test, excluding the previously selected companies. For example, if the original random number for a specific 3-Digit NAICS Category was 3 then the Pilot Test included companies 3, 13, 23, 33... The final test from this NAICS 3-Digit category will include $1, \ldots, 5,7,9,11, \ldots, 15 \ldots$

\section{Dissertation Survey Results}

Results from the Pilot Test are of sufficient quality to be included with the Dissertation Survey for combined results analysis. A total of 3,578 companies are selected based on a 50\% sampling of all active publicly traded companies. Of these companies, 2,217 are publicly traded for 5 years or more, of which 1,732 have email and contact information available. This comprised the sample set for survey distribution. The Survey e-mail Campaign is scheduled as follows: 
- Week 1 -- (09/10/09) Th 1st Contact Email Prior Letter

- Week 2 -- (09/14/09) Mon 2nd Contact Survey Cover Letter

- Week 3 -- (09/21/09) Mon 3rd Contact E-mail Thank You/Reminder

- Week 5 -- (10/05/09) Mon 4th Contact Repeat Questionnaire

- Week 10 -- (11/09/09) Mon 5th Final Contact

The actual campaign schedule had to be modified due to a power outage resulting in the survey instrument being inaccessible for Week $5(10 / 05 / 09)$, so distribution was delayed until 10/14/09 (Appendix K). Based on the number of actively traded companies in each NAICS category from which email and contact information is available a total of 64 NAICS categories are analyzed. If fewer than 3 contacts are available no testing is done (Categories 238, 483, and 711 are not analyzed due to insufficient number of contacts for dependent variable definition). 29 (45.31\%) of the 64 NAICS categories included responding companies. Table 41 shows the response rate for each of the NAICS categories: 
TABLE 41

NAICS CATEGORY RESPONSES

\begin{tabular}{|c|c|c|c|c|c|c|c|}
\hline $\begin{array}{l}3 \text { Digit } \\
\text { NAlOs } \\
\text { Code }\end{array}$ & $\begin{array}{l}\text { \# Contacts } \\
5 \text { yrs } \\
\text { wi email }\end{array}$ & Responses & $\begin{array}{l}\text { Response } \\
\text { Rate }\end{array}$ & $\begin{array}{l}3 \text { Digit } \\
\text { Wás } \\
\text { Gode }\end{array}$ & $\begin{array}{l}\# \\
\text { Contacts } \\
5 \text { yrs } \\
\text { w'email }\end{array}$ & Fesponses & $\begin{array}{l}\text { Response } \\
\text { Rate }\end{array}$ \\
\hline 211 & 30 & 2 & 6.678 & 452 & 6 & & \\
\hline 212 & 13 & & & 453 & 7 & & \\
\hline 213 & 20 & & & 454 & 6 & & \\
\hline 221 & 49 & 5 & $10.20 \%$ & 481 & 7 & & \\
\hline 236 & 13 & & & 483 & 1 & & \\
\hline 237 & 9 & 2 & $22.22 \%$ & 484 & 10 & & \\
\hline 238 & 2 & & & 486 & 9 & & \\
\hline 311 & 25 & 3 & $12.00 \%$ & 489 & 4 & & \\
\hline 312 & 10 & 1 & $10.00 \%$ & 511 & 59 & 3 & $5.08 \%$ \\
\hline 315 & 10 & & & 512 & 5 & & \\
\hline 316 & 7 & & & 515 & 12 & 1 & 8.336 \\
\hline 321 & 4 & & & 517 & 25 & & \\
\hline 322 & 12 & 2 & $16.67 \%$ & 518 & 21 & & \\
\hline 323 & 6 & & & 522 & 221 & 14 & $6.33 \%$ \\
\hline 324 & 6 & & & 523 & 33 & & \\
\hline 325 & 158 & 6 & $3.80 \%$ & 524 & 45 & 1 & 2.228 \\
\hline 326 & 11 & 1 & 9.096 & 525 & 56 & 2 & 3.578 \\
\hline 327 & 4 & & & 531 & 9 & & \\
\hline 331 & 10 & & & 532 & 7 & & \\
\hline 332 & 23 & 1 & 4.358 & 533 & 9 & 1 & $11.11 \%$ \\
\hline 333 & 54 & 4 & 7.418 & 541 & 104 & 4 & $3.85 \%$ \\
\hline 334 & 245 & 8 & $3.27 \%$ & 551 & 12 & & \\
\hline 335 & 24 & 1 & 4.178 & 561 & 35 & 1 & $2.86 \%$ \\
\hline 336 & 34 & 2 & 5.988 & 562 & 7 & 1 & $14.29 \%$ \\
\hline 337 & 9 & & & 611 & 10 & & \\
\hline 339 & 58 & 1 & 1.728 & 621 & 21 & & \\
\hline 423 & 33 & 1 & 3.036 & 622 & 4 & & \\
\hline 424 & 16 & & & 623 & 3 & & \\
\hline 441 & 7 & 1 & $14.29 \%$ & 711 & 1 & & \\
\hline 443 & 3 & 1 & $33.33 \%$ & 713 & 13 & 1 & 7.698 \\
\hline 445 & 4 & & & 721 & 5 & & \\
\hline 446 & 5 & & & 722 & 20 & 1 & $5.010 \%$ \\
\hline 448 & 16 & & & 812 & 11 & 2 & $18.18 \%$ \\
\hline 451 & 4 & & & & & & \\
\hline \multicolumn{5}{|l|}{ Total } & 1732 & 74 & \\
\hline
\end{tabular}

A total sample size of 76 (74 companies) is achieved compared to the expected 80 based on the Pilot Test. One respondent indicated company number 9999.999 which was the example code number and could not be identified, and one company submitted two responses as was requested in the Pilot Test. Table 42 summarizes the combined survey response results: 
TABLE 42

COMBINED SURVEY RESPONSE RESULTS

\begin{tabular}{|c|c|c|c|c|c|c|}
\hline \multicolumn{7}{|c|}{ Pilot Test and Dissertation Survey Contacts Setup } \\
\hline \multirow{4}{*}{$\begin{array}{l}50 \% \text { Sampling } \\
5 \text { Years Flus } \\
\text { Wi email contact info }\end{array}$} & Filot & Percent & Survey & Percent & Cumulative & Percent \\
\hline & 701 & $100.010 \%$ & 2077 & $100.00 \%$ & 3578 & $100.00 \%$ \\
\hline & 452 & $64.48 \%$ & 1765 & $61.35 \%$ & 2217 & $61.96 \%$ \\
\hline & 360 & $51.36 \%$ & 1372 & $47.69 \%$ & 1732 & $48.41 \%$ \\
\hline \multicolumn{7}{|c|}{$\begin{array}{l}\text { Dissertation Euwey Notes: } \\
4 \text { eliminated due to insufficient contacts to define dependent variable [1372-4=1368] } \\
8 \text { consistenty not delwered by campaign so contacts accordingly reduced [1368 } 8=1360]\end{array}$} \\
\hline \multicolumn{7}{|c|}{ Pilot Test Survey Results } \\
\hline Pilot Test & Contacts & Remove & $\begin{array}{c}\text { Survey } \\
\text { completion }\end{array}$ & $\begin{array}{l}\text { Percent of } \\
\text { Distribution } \\
\text { Compilete }\end{array}$ & $\begin{array}{c}\text { Percent of } \\
\text { Total } \\
\text { Contacts } \\
\text { Complete }\end{array}$ & \\
\hline Pilat Test 1 & 360 & 8 & & & & \\
\hline *Pilot Test 2 & 352 & 2 & 7 & $1.99 \%$ & $1.94 \%$ & \\
\hline Filot Test 3 & 344 & 11 & 1 & $0.29 \%$ & $0.28 \%$ & \\
\hline Filot Test 4 & 332 & 3 & 5 & $1.51 \%$ & $1.39 \%$ & \\
\hline Pilot Test 5 & 324 & & 3 & $0.93 \%$ & $0.83 \%$ & \\
\hline Total & & 24 & 16 & & $4.44 \%$ & \\
\hline * 6 companies completed & & $6.67 \%$ & & & & \\
\hline \multicolumn{7}{|c|}{ Dissertation Survey Results } \\
\hline Dissertation Surwey & Contacts & Remove & $\begin{array}{c}\text { Survey } \\
\text { completion }\end{array}$ & $\begin{array}{l}\text { Percent of } \\
\text { Distribution } \\
\text { Complete }\end{array}$ & $\begin{array}{c}\text { Percent of } \\
\text { Total } \\
\text { Contacts } \\
\text { Complete }\end{array}$ & \\
\hline Disertation Survey 1 & 1360 & 7 & & & & \\
\hline Dissertation Sure/ 2 & 1353 & 10 & 28 & $2.07 \%$ & $2.06 \%$ & \\
\hline Dissertation Suney 3 & 1315 & 13 & 16 & $1.22 \%$ & $1.18 \%$ & \\
\hline "Dissertation Surwey 4 & 1286 & 19 & 12 & $0.93 \%$ & $0.88 \%$ & \\
\hline Dissertation Survey 5 & 1256 & & 4 & $0.32 \%$ & $0.29 \%$ & \\
\hline Total & & 49 & 60 & & $4.41 \%$ & \\
\hline \multicolumn{7}{|c|}{$\begin{array}{l}\text { "Distribution delay ed } 9 \text { days - one completed as code } 9999.999 \text { not usable } \\
\text { "SsL Server went down seweral hours day of distribution, one message from contact security not good }\end{array}$} \\
\hline \multicolumn{7}{|c|}{ Cumulative Survey Results } \\
\hline Dissertation Surwey & Contacts & Remove & $\begin{array}{c}\text { Survey } \\
\text { completion }\end{array}$ & $\begin{array}{l}\text { Percent of } \\
\text { Distribution } \\
\text { Complete }\end{array}$ & $\begin{array}{c}\text { Percent of } \\
\text { Total } \\
\text { Contacts } \\
\text { Compilete }\end{array}$ & \\
\hline omulative 1 & 1720 & 15 & $\overline{0}$ & $\overline{0}$ & $\overline{0}$ & \\
\hline Cumulative 2 & 1705 & 12 & 35 & $2.05 \%$ & $2.03 \%$ & \\
\hline Cumulative 3 & 1659 & 24 & 17 & $1.02 \%$ & $0.99 \%$ & \\
\hline Cumulative 4 & 1618 & 22 & 17 & $1.05 \%$ & $0.99 \%$ & \\
\hline Oumulative 5 & 1580 & 0 & 7 & $0.44 \%$ & $0.41 \%$ & \\
\hline Total Cumulative & & $\begin{array}{r}73 \\
4.24 \%\end{array}$ & 76 & & $4.42 \%$ & \\
\hline
\end{tabular}


Responses to the Survey are documented (Appendix L) by ID as chronologically submitted $(1-76)$.

\section{Hypothesis Testing}

The dependent variable is the 12-month rolling average stock price comparisons of 1/05 vs. 1/09 for all sample companies in each of the 29 responding NAICS categories (1,288 companies with data available). The begin date of 1/05 for the comparison date range requires that every company is publicly traded for $\geq 5$ years since only companies for which monthly stock price history is available as of 2/2/04 are included (1/05 result is monthly average of $2 / 2 / 04$ to $1 / 3 / 05$ and the comparison $1 / 09$ result is monthly average of 2/1/08 to 1/2/09; data range for each company is therefore February 2004 through January 2009).

Appendix M shows the years the companies are publicly traded and percentage change for all sample companies in the NAICS categories with responses. Data are sorted by: 1) NAICS 3-Digit Code; 2) Percent Change in Stock Price One-Year Rolling Average from 1/05 to 1/09. Each 3-Digit NAICS category is segmented into thirds to define low, medium and high performers. Respondent companies are highlighted and pilot test solicitations are noted. The methodology is demonstrated for NAICS Code 211 in Table 43 as follows: 
TABLE 43

DEPENDENT VARIABLE DEFINITION NAICS CATEGORY 211

\begin{tabular}{|c|c|c|c|c|c|c|c|c|c|c|c|}
\hline Pilot & NACS & Comp & $\begin{array}{l}12 / 08 \\
0 \text { Age yrs. }\end{array}$ & $\begin{array}{l}\text { \% Change } \\
1,05-1,09\end{array}$ & $\begin{array}{l}\text { HLM } \\
\text { Groupings }\end{array}$ & $\begin{array}{l}\text { Rank } \\
\text { HLM }\end{array}$ & Count & Mean & Std.Dev & $\operatorname{Max}$ & Min \\
\hline & 211 & 060 & 6.8 & -66.05 & & & & & & & \\
\hline & 211 & 080 & 13.3 & -64.15 & & & & & & & \\
\hline & 211 & 134 & 8.5 & -55.52 & & & & & & & \\
\hline & 211 & 142 & 14.1 & -46.47 & & & & & & & \\
\hline & 211 & 160 & 16.8 & -27.46 & & & & & & & \\
\hline & 211 & 076 & 7.6 & -21.95 & & & & & & & \\
\hline \multirow[t]{2}{*}{$\mathrm{P}$} & 211 & 098 & 13.3 & -18.97 & & & & & & & \\
\hline & 211 & 162 & 8.5 & -7.82 & & & & & & & \\
\hline \multirow[t]{8}{*}{$\bar{P}$} & 211 & 048 & 15.2 & 22.58 & & & & & & & \\
\hline & 211 & 114 & 11.9 & 37.44 & 10 & & & & & & \\
\hline & 211 & 010 & 11.9 & 38.72 & & & & & & & \\
\hline & 211 & 126 & 11.3 & 39.12 & & & & & & & \\
\hline & 211 & 166 & 20.9 & 62.58 & & & & & & & \\
\hline & 211 & 004 & 11.9 & 66.13 & & & & & & & \\
\hline & 211 & 154 & 17.4 & 73.52 & & & & & & & \\
\hline & 211 & 146 & 16.0 & 100.01 & & m & & & & & \\
\hline \multirow[t]{2}{*}{$\mathrm{P}$} & 211 & 168 & 11.9 & 106.04 & & & & & & & \\
\hline & 211 & 022 & 21.4 & 110.51 & & & & & & & \\
\hline $\mathrm{P}$ & 211 & 128 & 6.0 & 111.10 & & & & & & & \\
\hline \multirow[t]{2}{*}{$\mathrm{P}$} & 211 & 008 & 27.0 & 134.68 & 10 & & & & & & \\
\hline & 211 & 136 & 16.8 & 139.27 & & & & & & & \\
\hline $\mathrm{P}$ & 211 & 038 & 18.8 & 149.23 & & & & & & & \\
\hline \multirow[t]{3}{*}{$P$} & 211 & 118 & 14.8 & 160.45 & & & & & & & \\
\hline & 211 & 112 & 26.9 & 166.54 & & & & & & & \\
\hline & 211 & 034 & 15.8 & 166.75 & & & & & & & \\
\hline \multirow[t]{6}{*}{$\mathrm{P}$} & 211 & 028 & 18.8 & 225.60 & & & & & & & \\
\hline & 211 & 064 & 19.2 & 241.53 & & & & & & & \\
\hline & 211 & 082 & 21.0 & 249.68 & & & & & & & \\
\hline & 211 & 090 & 6.9 & 530.01 & & & & & & & \\
\hline & 211 & 040 & 9.2 & 837.52 & 10 & & 30 & 115.35 & 183.42 & 837.52 & -66.05 \\
\hline & 221 & $\overline{047}$ & 5.2 & -74.24 & & & & & & & \\
\hline
\end{tabular}

This analysis also is performed based on "responding companies only" (Appendix $\mathrm{N})$ for comparison of respondent performance rankings with and without the standardization based on NAICS 3-Digit Category as follows: 
TABLE 44

\section{DEPENDENT VARIABLE DEFINITION}

\begin{tabular}{|c|c|c|c|c|}
\hline Pilot & $\begin{array}{l}\text { NAlCS } \\
\text { Code }\end{array}$ & $\begin{array}{l}\text { Co. } \\
\text { No. }\end{array}$ & $\begin{array}{l}\text { NAICS } \\
1,05- \\
1,09\end{array}$ & $\begin{array}{l}\text { Responding } \\
1,05- \\
1,09\end{array}$ \\
\hline & 211 & 060 & $L$ & $L$ \\
\hline & 211 & 146 & $M$ & $\mathrm{H}$ \\
\hline & 221 & 001 & $\mathrm{~L}$ & $L$ \\
\hline & 221 & 049 & $L$ & $\mathrm{M}$ \\
\hline & 221 & 101 & $\mathrm{H}$ & $\mathrm{H}$ \\
\hline \multirow[t]{2}{*}{$\bar{P}$} & 221 & 105 & $\mathrm{M}$ & $\mathrm{H}$ \\
\hline & 221 & 141 & $M$ & $\mathrm{H}$ \\
\hline \multirow[t]{2}{*}{$\bar{P}$} & 237 & 011 & $M$ & $M$ \\
\hline & 237 & 027 & $\mathrm{~L}$ & $\mathrm{~L}$ \\
\hline$P$ & 311 & 0.38 & $\mathrm{H}$ & $\mathrm{H}$ \\
\hline \multirow[t]{6}{*}{$P$} & 311 & 058 & $\mathrm{~L}$ & $L$ \\
\hline & 311 & 084 & $\mathrm{~L}$ & $M$ \\
\hline & 312 & 033 & $\mathrm{~L}$ & $\mathrm{~L}$ \\
\hline & 322 & 006 & \multicolumn{2}{|c|}{ CHAPTER 11} \\
\hline & 322 & 026 & $\mathrm{H}$ & $\mathrm{H}$ \\
\hline & 325 & 0.35 & $\mathrm{~L}$ & $L$ \\
\hline \multirow[t]{2}{*}{$\mathrm{P}$} & 325 & 153 & $\mathrm{~L}$ & $\mathrm{~L}$ \\
\hline & 325 & 277 & $\mathrm{M}$ & $M$ \\
\hline \multirow[t]{2}{*}{$\mathrm{P}$} & 325 & 303 & $\mathrm{H}$ & $\mathrm{M}$ \\
\hline & 325 & 449 & $\mathrm{H}$ & $\mathrm{H}$ \\
\hline \multirow[t]{16}{*}{$\bar{P}$} & 325 & 453 & $M$ & $\mathrm{~L}$ \\
\hline & 326 & 0.36 & $\mathrm{H}$ & $\mathrm{H}$ \\
\hline & 332 & 007 & $\mathrm{~L}$ & $M$ \\
\hline & 333 & 070 & $\mathrm{H}$ & $\mathrm{H}$ \\
\hline & 333 & 104 & $\mathrm{~L}$ & $M$ \\
\hline & 333 & 130 & $\bar{L}$ & $\bar{L}$ \\
\hline & 333 & 146 & $M$ & $\mathrm{H}$ \\
\hline & 334 & 131 & $\mathrm{H}$ & $M$ \\
\hline & 334 & 191 & $\overline{\mathrm{H}}$ & $\mathrm{H}$ \\
\hline & 334 & 229 & \multicolumn{2}{|c|}{ N/AFOR DATARANGE } \\
\hline & 334 & 421 & $\overline{\mathrm{H}}$ & $\overline{\mathrm{H}}$ \\
\hline & 334 & 439 & $\mathrm{M}$ & $\mathrm{L}$ \\
\hline & 334 & 477 & $\mathrm{H}$ & $M$ \\
\hline & 334 & 499 & $\mathrm{M}$ & $L$ \\
\hline & 334 & 601 & $M$ & $L$ \\
\hline & 335 & 009 & $M$ & $\mathrm{H}$ \\
\hline 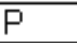 & 336 & 048 & $\mathrm{M}$ & $|\mathrm{M}|$ \\
\hline & 336 & 074 & $\mathrm{H}$ & $\mathrm{H}$ \\
\hline
\end{tabular}

\begin{tabular}{|c|c|c|c|c|}
\hline Pilot & $\begin{array}{l}\text { NAICS } \\
\text { Code }\end{array}$ & $\begin{array}{l}\text { Co. } \\
\text { No. }\end{array}$ & $\begin{array}{l}\text { N,ACS } \\
1,05- \\
1,09\end{array}$ & $\begin{array}{l}\text { Responding } \\
1,05- \\
1,09\end{array}$ \\
\hline & 339 & 087 & $\mathrm{M}$ & $M$ \\
\hline \multirow[t]{22}{*}{$\mathrm{P}$} & 423 & 135 & $\mathrm{M}$ & $M$ \\
\hline & 441 & 025 & $L$ & $\mathrm{~L}$ \\
\hline & 443 & 006 & $\mathrm{M}$ & $M$ \\
\hline & 511 & 005 & $\mathrm{M}$ & $\mathrm{M}$ \\
\hline & 511 & 019 & $\mathrm{M}$ & $M$ \\
\hline & 511 & 159 & $M$ & $M$ \\
\hline & 515 & 009 & $\mathrm{M}$ & $\mathrm{L}$ \\
\hline & 522 & 135 & $\mathrm{H}$ & $\mathrm{H}$ \\
\hline & 522 & 199 & $\mathrm{H}$ & $\mathrm{H}$ \\
\hline & 522 & 233 & $\mathrm{H}$ & $M$ \\
\hline & 522 & 315 & L & $L$ \\
\hline & 522 & 319 & $\mathrm{H}$ & $M$ \\
\hline & 522 & 337 & $\mathrm{M}$ & $\mathrm{L}$ \\
\hline & 522 & 349 & $\mathrm{H}$ & $M$ \\
\hline & 522 & 359 & $\mathrm{H}$ & $\mathrm{H}$ \\
\hline & 522 & 417 & $\mathrm{H}$ & $M$ \\
\hline & 522 & 449 & $\bar{L}$ & $\bar{L}$ \\
\hline & 522 & 499 & $M$ & $L$ \\
\hline & 522 & 625 & $\mathrm{~L}$ & $\bar{L}$ \\
\hline & 522 & 659 & $\mathrm{M}$ & $M 1$ \\
\hline & 522 & 679 & $\mathrm{H}$ & $\mathrm{M}$ \\
\hline & 524 & 074 & $\mathrm{H}$ & $\mathrm{H}$ \\
\hline \multirow[t]{4}{*}{$\mathrm{P}$} & 525 & 475 & $\mathrm{H}$ & $\mathrm{H}$ \\
\hline & 525 & 791 & $\mathrm{H}$ & $\mathrm{H}$ \\
\hline & 533 & 016 & $\mathrm{H}$ & $\mathrm{H}$ \\
\hline & 541 & 018 & $L$ & $L$ \\
\hline \multirow[t]{2}{*}{$\mathrm{P}$} & 541 & 052 & $L$ & $\mathrm{~L}$ \\
\hline & 541 & 150 & $\mathrm{M}$ & $M$ \\
\hline \multirow[t]{3}{*}{$P$} & 541 & 352 & $\mathrm{H}$ & $\mathrm{H}$ \\
\hline & 561 & 078 & $\mathrm{M}$ & $M$ \\
\hline & 562 & 030 & $\mathrm{M}$ & $L$ \\
\hline $\bar{P}$ & 713 & 039 & $L$ & $\bar{L}$ \\
\hline $\mathrm{P}$ & 722 & 054 & $L$ & $\mathrm{~L}$ \\
\hline \multirow[t]{2}{*}{$P$} & 812 & 0106 & $\bar{H}$ & $\vec{H}$ \\
\hline & 812 & 028 & $\overline{\mathrm{H}}$ & $\mathrm{H}$ \\
\hline \multirow[t]{3}{*}{ Coun } & & $\mathrm{H}$ & 27 & 2 \\
\hline & & $\mathrm{M}$ & 25 & 2 \\
\hline & & $\mathrm{L}$ & 20 & 2 \\
\hline
\end{tabular}


IBM SPSS Statistics GradPack 18 Windows (PASW Statistics GradPack) is used for testing.

Prior to Hypothesis testing four responses are deleted as follows relative to Respondent IDs noted in Appendix L: ID 1) No responses; ID 27) Unable to define Dependent Variable due to Chapter 11 filing; ID 70) Respondent used Company Code 9999.999, unable to identify company; and ID 72) Unable to define Dependent Variable, "Not Available for Data Range" message when attempting to download stock price histories. Data with these modifications is used for the Pre-Adjustment Results for each hypothesis.

Two responses are received from Company Code 4230.135: ID 3 Front Line Supervisor; and ID 5 Executive. The Executive position is typical of other company respondents, but to assure that results are not skewed based on this double company entry tests are performed based on: 1) Inclusion of both responses for this company identified as "Total Results"; 2) Inclusion of Executive response only for this company identified as "Executive Results"; and 3) Inclusion of Front Line Supervisor response only for this company identified as "Supervisor Results." Data is adjusted and is defined for each hypothesis to remove non-responses relative to the specific hypothesis in question. Results for each hypothesis are presented as: 1) Stated Hypothesis; 2) Pre-Adjustment results; 3) Description of the adjustments made for the specific hypothesis; 4) Total Results; 5) Executive Results; then 6) Supervisor Results followed by conclusions. 
Hypothesis 1 Test:

$\mathrm{H}_{1}$ : Salience of business processes identified for measurement correlate to firm value as tested by Kruskal-Wallis one-way ANOVA.

Prior to performing this test, data is transposed from Columns as rank 1, 2, 3, 4 with Row inputs Identity (I), Priority (P), Background (B), Mandated (M) to Columns as I, P, B, M and Rows as rank 1, 2, 3, 4:

Hypothesis Test Summary

\begin{tabular}{|c|c|c|c|c|}
\hline & Null Hypothesis & Test & Sig. & Decision \\
\hline 1 & $\begin{array}{l}\text { The distribution of Identity Process } \\
\text { Rank is the same across } \\
\text { categories of } 1 \mathrm{H}, 2 \mathrm{M}, 3 \mathrm{~L} \text { Rank. }\end{array}$ & $\begin{array}{l}\text { Independent- } \\
\text { Samples } \\
\text { Kruskal- } \\
\text { Wallis Test }\end{array}$ & .601 & $\begin{array}{l}\text { Retain the } \\
\text { null } \\
\text { hypothesis }\end{array}$ \\
\hline 2 & $\begin{array}{l}\text { The distribution of Priority Process } \\
\text { Rank is the same across } \\
\text { categories of } 1 \mathrm{H}, 2 \mathrm{M}, 3 \mathrm{~L} \text { Rank. }\end{array}$ & $\begin{array}{l}\text { Independent- } \\
\text { Samples } \\
\text { Kruskal- } \\
\text { Wallis Test }\end{array}$ & .659 & $\begin{array}{l}\text { Retain the } \\
\text { null } \\
\text { hypothesis }\end{array}$ \\
\hline 3 & $\begin{array}{l}\text { The distribution of Background } \\
\text { Process Rank is the same across } \\
\text { categories of } 1 \mathrm{H}, 2 \mathrm{M}, 3 \mathrm{~L} \text { Rank. }\end{array}$ & $\begin{array}{l}\text { Independent- } \\
\text { Samples } \\
\text { Kruskal- } \\
\text { Wallis Test }\end{array}$ & .961 & $\begin{array}{l}\text { Retain the } \\
\text { null } \\
\text { hypothesis }\end{array}$ \\
\hline
\end{tabular}

Asymptotic significances are displayed. The significance level is .05 .

\section{Figure 9: $H_{1}$ Pre-Adjustment Results}




\section{$\underline{\mathrm{H}}_{1} \underline{\text { Adjustments }}$}

$\mathrm{H}_{1}$ Test Data: Delete ID 28 No response.

$\mathrm{H}_{1}$ Executive: Delete ID 3 Supervisor.

$\mathrm{H}_{1}$ Supervisor: Delete ID 5 Executive.

Include Partial responses ID 69 and ID 75 -- assign both priority rank $=5$ for missing rankings based on logic that non-use ranks lower in priority than the defined lowest rank (Appendix O).

TABLE 45

\section{$\mathrm{H}_{1} \quad$ KRUSKAL-WALLIS MEANS}

\begin{tabular}{|l|r|r|r|}
\hline & \multicolumn{1}{|c|}{ Low } & Medium & High \\
\hline Total Identity & 37.00 & 33.60 & 37.52 \\
\hline Total Priority & 34.66 & 39.68 & 33.54 \\
\hline Total Background & 35.84 & 36.86 & 35.31 \\
\hline & & & \\
\hline & Low & Medium & High \\
\hline Executive Identity & 36.47 & 33.04 & 37.00 \\
\hline Executive Priority & 33.79 & 40.00 & 32.70 \\
\hline Executive Background & 35.29 & 36.46 & 34.80 \\
\hline & & & \\
\hline & Low & Medium & High \\
\hline Supervisor Identity & 36.47 & 33.04 & 37.00 \\
\hline Supervisor Priority & 34.61 & 38.46 & 33.50 \\
\hline Supervisor Background & 35.29 & 36.46 & 34.8 \\
\hline
\end{tabular}




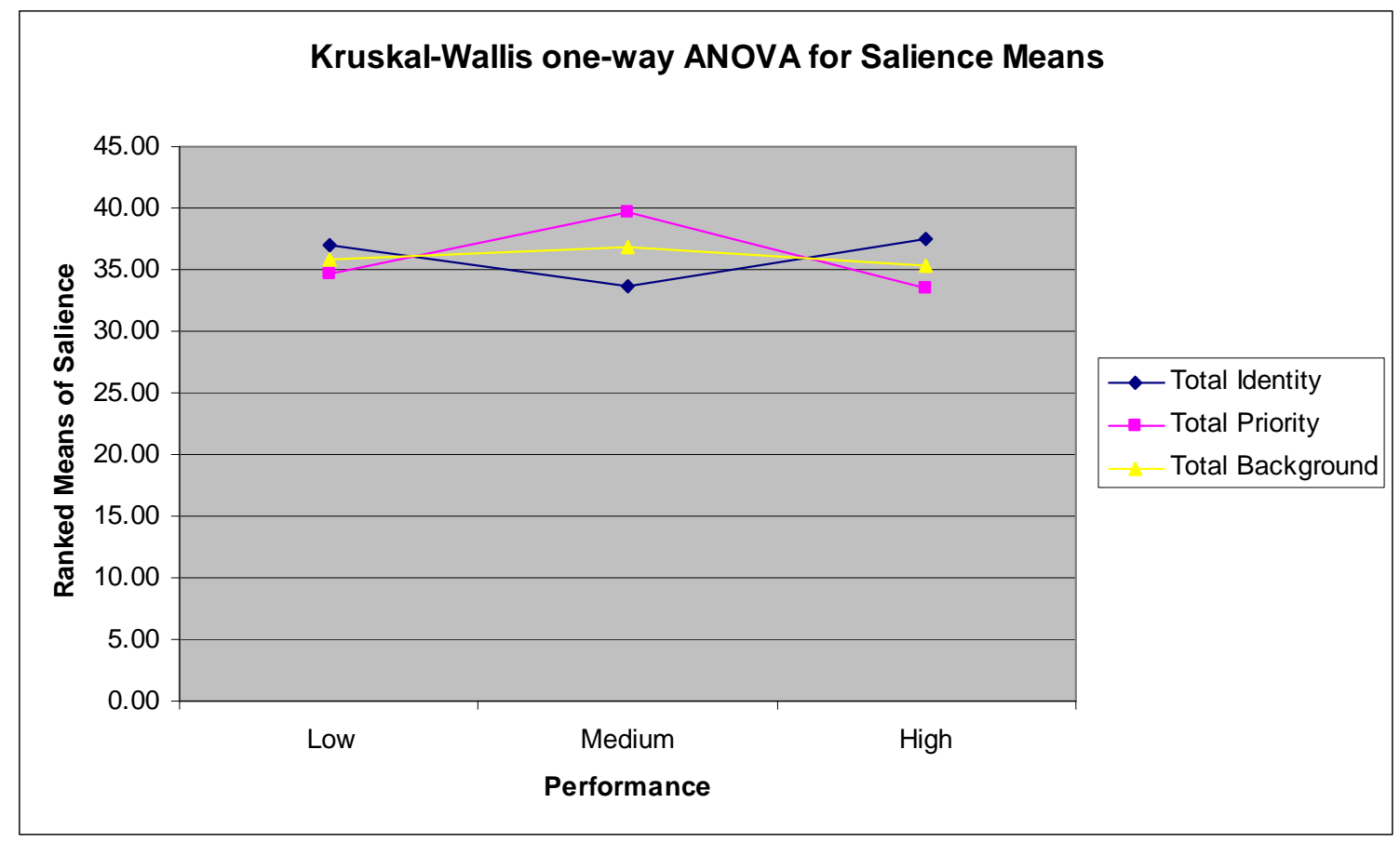

Hypothesis Test Summary

\begin{tabular}{|llll|}
\hline \multicolumn{1}{|c}{ Null Hypothesis } & \multicolumn{1}{c}{ Test } & Sig. & Decision \\
\hline $\mathbf{1}$ & $\begin{array}{l}\text { The distribution of Identity Process } \\
\text { Rank is the same across } \\
\text { categories of } 1 \mathrm{H}, 2 \mathrm{M}, 3 \mathrm{~L} \text { Rank. }\end{array}$ & $\begin{array}{l}\text { Independent- } \\
\text { Samples } \\
\text { Kruskal- } \\
\text { Wallis Test }\end{array}$ & $.752 \begin{array}{l}\text { Retain the } \\
\text { null } \\
\text { hypothesis. }\end{array}$ \\
\hline $\begin{array}{l}\text { The distribution of Priority Process } \\
\text { Rank is the same across } \\
\text { categories of } 1 \mathrm{H}, 2 \mathrm{M}, 3 \mathrm{~L} \text { Rank. }\end{array}$ & $\begin{array}{l}\text { Independent- } \\
\text { Samples } \\
\text { Kruskal- } \\
\text { Wallis Test }\end{array}$ & $.503 \begin{array}{l}\text { Retain the } \\
\text { null } \\
\text { hypothesis. }\end{array}$ \\
\hline $\mathbf{3}$ The distribution of Background \\
$\begin{array}{l}\text { Process Rank is the same across } \\
\text { categories of } 1 \mathrm{H}, 2 \mathrm{M}, 3 \mathrm{~L} \text { Rank. }\end{array}$ & $\begin{array}{l}\text { Independent- } \\
\text { Samples } \\
\text { Kruskal- } \\
\text { Wallis Test }\end{array}$ & .961 & $\begin{array}{l}\text { Retain the } \\
\text { null } \\
\text { hypothesis. }\end{array}$ \\
\hline
\end{tabular}

Asymptotic significances are displayed. The significance level is .05 .

Figure 10: $H_{1}$ Total Results 


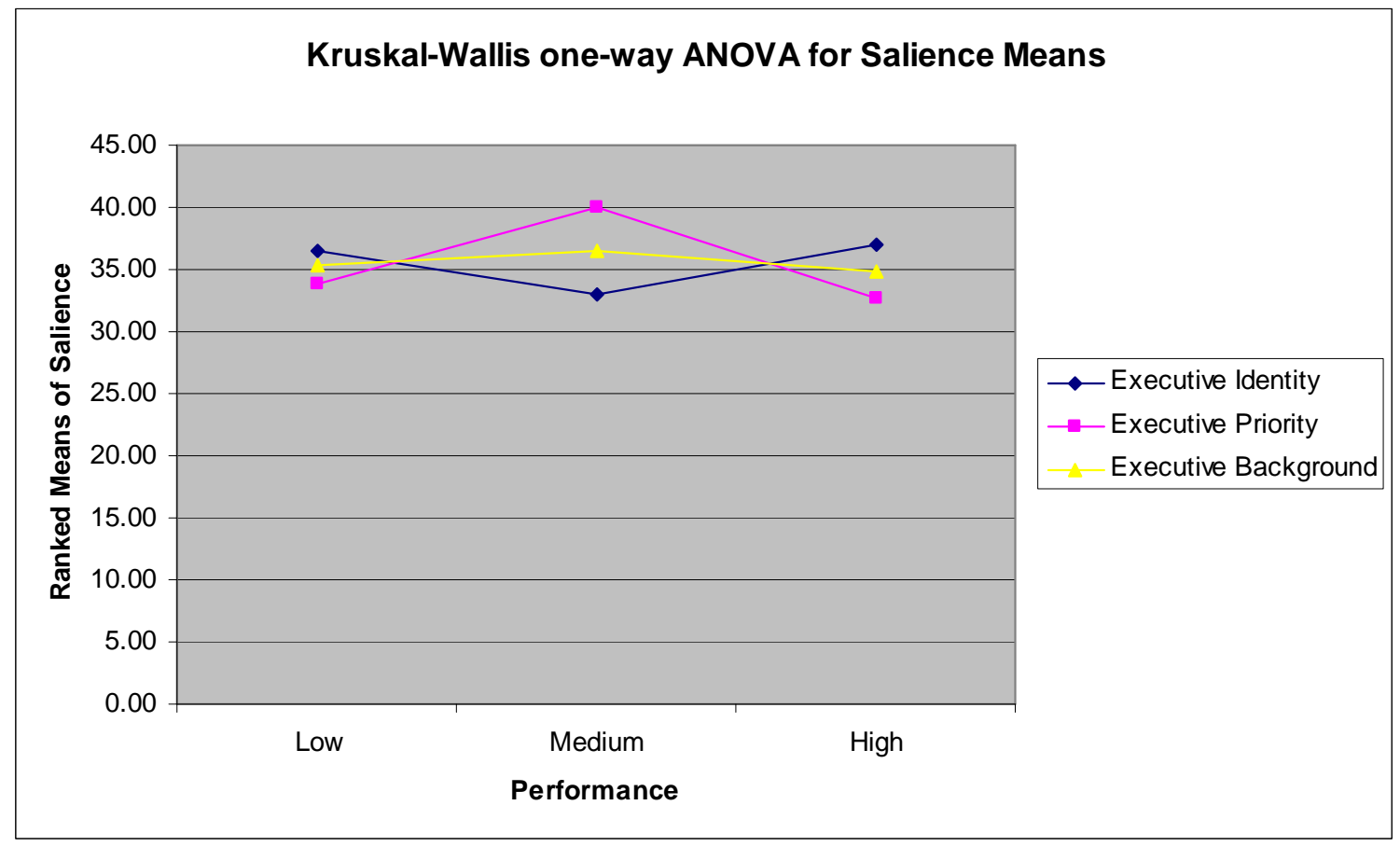

Hypothesis Test Summary

\begin{tabular}{|c|c|c|c|c|}
\hline & Null Hypothesis & Test & Sig. & Decision \\
\hline 1 & $\begin{array}{l}\text { The distribution of Identity Process } \\
\text { Rank is the same across } \\
\text { categories of } 1 \mathrm{H}, 2 \mathrm{M}, 3 \mathrm{~L} \text { Rank. }\end{array}$ & $\begin{array}{l}\text { Independent- } \\
\text { Samples } \\
\text { Kruskal- } \\
\text { Wallis Test }\end{array}$ & .747 & $\begin{array}{l}\text { Retain the } \\
\text { null } \\
\text { hypothesis }\end{array}$ \\
\hline 2 & $\begin{array}{l}\text { The distribution of Priority Process } \\
\text { Rank is the same across } \\
\text { categories of } 1 \mathrm{H}, 2 \mathrm{M}, 3 \mathrm{~L} \text { Rank. }\end{array}$ & $\begin{array}{l}\text { Independent- } \\
\text { Samples } \\
\text { Kruskal- } \\
\text { Wallis Test }\end{array}$ & .371 & $\begin{array}{l}\text { Retain the } \\
\text { null } \\
\text { hypothesis }\end{array}$ \\
\hline 3 & $\begin{array}{l}\text { The distribution of Background } \\
\text { Process Rank is the same across } \\
\text { categories of } 1 H, 2 M, 3 L \text { Rank. }\end{array}$ & $\begin{array}{l}\text { Independent- } \\
\text { Samples } \\
\text { Kruskal- } \\
\text { Wallis Test }\end{array}$ & .954 & $\begin{array}{l}\text { Retain the } \\
\text { null } \\
\text { hypothesis }\end{array}$ \\
\hline
\end{tabular}

Asymptotic significances are displayed. The significance level is .05 .

\section{Figure 11: $H_{1}$ Executive Results}




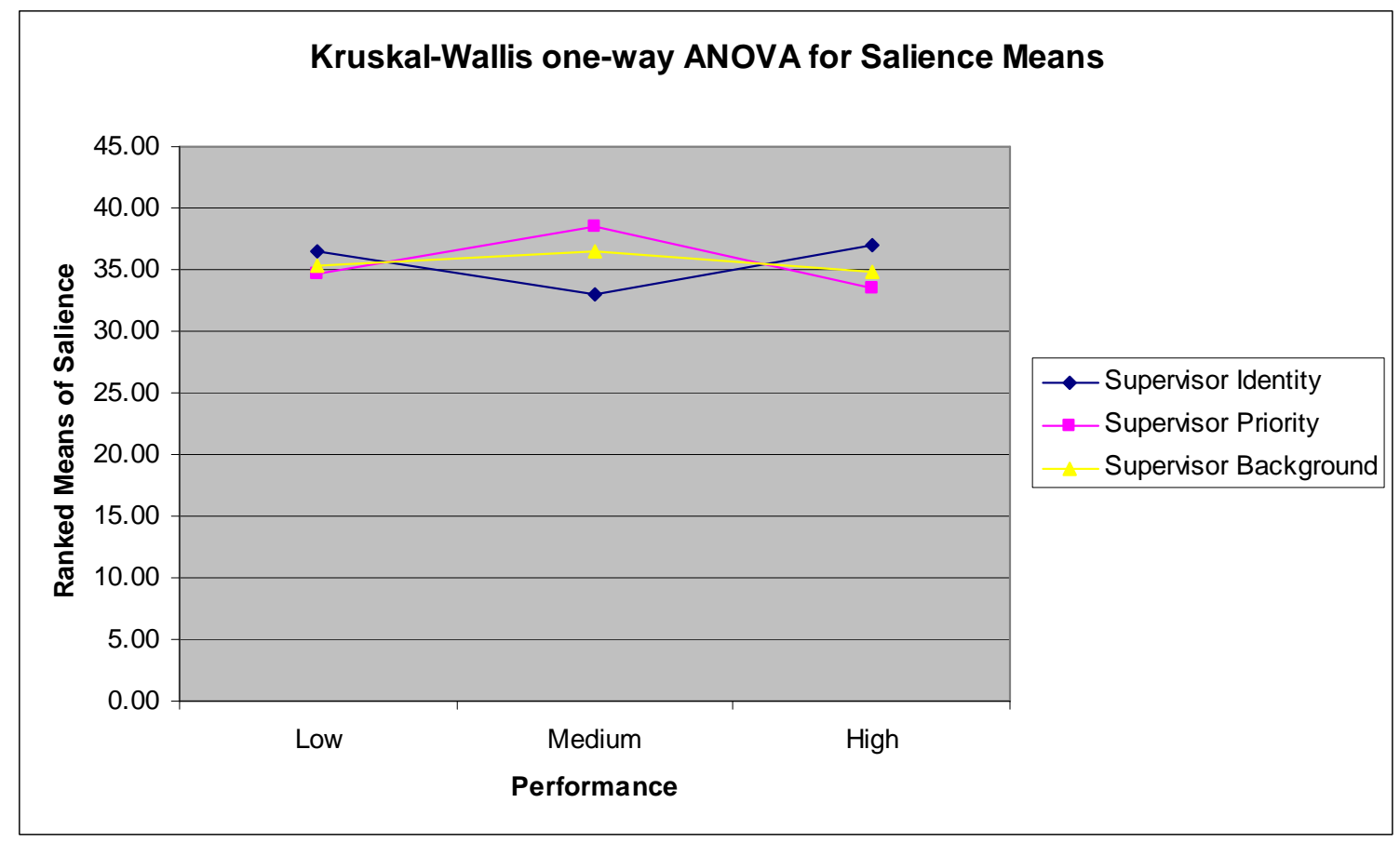

Hypothesis Test Summary

\begin{tabular}{|c|c|c|c|c|}
\hline & Null Hypothesis & Test & Sig. & Decision \\
\hline 1 & $\begin{array}{l}\text { The distribution of Identity Process } \\
\text { Rank is the same across } \\
\text { categories of } 1 \mathrm{H}, 2 \mathrm{M}, 3 \mathrm{~L} \text { Rank. }\end{array}$ & $\begin{array}{l}\text { Independent- } \\
\text { Samples } \\
\text { Kruskal- } \\
\text { Wallis Test }\end{array}$ & .747 & $\begin{array}{l}\text { Retain the } \\
\text { null } \\
\text { hypothesis }\end{array}$ \\
\hline 2 & $\begin{array}{l}\text { The distribution of Priority Process } \\
\text { Rank is the same across } \\
\text { categories of } 1 \mathrm{H}, 2 \mathrm{M}, 3 \mathrm{~L} \text { Rank. }\end{array}$ & $\begin{array}{l}\text { Independent- } \\
\text { Samples } \\
\text { Kruskal- } \\
\text { Wallis Test }\end{array}$ & .644 & $\begin{array}{l}\text { Retain the } \\
\text { null } \\
\text { hypothesis }\end{array}$ \\
\hline 3 & $\begin{array}{l}\text { The distribution of Background } \\
\text { Process Rank is the same across } \\
\text { categories of } 1 \mathrm{H}, 2 \mathrm{M}, 3 \mathrm{~L} \text { Rank. }\end{array}$ & $\begin{array}{l}\text { Independent- } \\
\text { Samples } \\
\text { Kruskal- } \\
\text { Wallis Test }\end{array}$ & .954 & $\begin{array}{l}\text { Retain the } \\
\text { null } \\
\text { hypothesis. }\end{array}$ \\
\hline
\end{tabular}

Asymptotic significances are displayed. The significance level is .05.

\section{Figure 12: $H_{1}$ Supervisor Results}


$\mathrm{H}_{1 \mathrm{a}}$ : Identity business processes using the means results from $\mathrm{H}_{1}$ have a higher positive correlation to firm value than Priority business processes as tested by the Chi-Square Contingency Test of Association.

TABLE 46

\section{$\mathrm{H}_{1 \mathrm{a}}$ CHI-SQUARE CONTINGENCY TEST IDENTITY VS PRIORITY}

Process * Performance Crosstabulation

Count

\begin{tabular}{|rl|r|r|r|r|}
\hline & & \multicolumn{3}{|c|}{ Performance } & \multirow{2}{*}{ Total } \\
\cline { 3 - 5 } & & \multicolumn{1}{|c|}{$1 \mathrm{H}$} & \multicolumn{1}{c|}{$2 \mathrm{Wi}$} & \multicolumn{1}{c|}{$3 \mathrm{~L}$} & \multicolumn{1}{c|}{109} \\
\hline Process & $\mathrm{I}$ & 38 & 34 & 37 & 109 \\
& $\mathrm{P}$ & 34 & 40 & 35 & 218 \\
\hline
\end{tabular}

Chi-Square Tests

\begin{tabular}{|l|r|r|r|}
\hline & Value & df & \multicolumn{1}{c|}{$\begin{array}{c}\text { Asymp. Sig. } \\
(2-s i d e d)\end{array}$} \\
\hline Pearson Chi-Square & $.764^{3}$ & 2 & .682 \\
Likelihood Ratio & .765 & 2 & .682 \\
N of valid Cases & 218 & & \\
\hline
\end{tabular}

a. 0 cells (0\%) have expected count less than 5 . The minimum expected count is 36.00 .

Symumetric Measures

\begin{tabular}{|l|r|r|}
\hline & Value & Approx Sig. \\
\hline Nominal by Nominal Contingency Coeficient & .059 & .682 \\
N of Valid Cases & 218 & \\
\hline
\end{tabular}


$\mathrm{H}_{1 \mathrm{~b}}$ : Priority business processes using the means results from $\mathrm{H}_{1}$ have a higher positive correlation to firm value than Background business processes as tested by the Chi-Square Contingency Test of Association.

TABLE 47

$\mathrm{H}_{1 \mathrm{~b}}$ CHI-SQUARE CONTINGENCY TEST PRIORITY VS BACKGROUND

Process * Performance Crosstabulation

Count

\begin{tabular}{|ll|r|r|r|r|}
\hline & & \multicolumn{3}{|c|}{ Performance } & \multirow{2}{*}{ Total } \\
\cline { 3 - 5 } & & \multicolumn{1}{|c|}{$1 \mathrm{H}$} & \multicolumn{1}{|c|}{$2 \mathrm{M}$} & \multicolumn{1}{c|}{$3 \mathrm{~L}$} & 108 \\
\hline Frocess & $\mathrm{B}$ & 35 & 37 & 36 & 108 \\
& $\mathrm{P}$ & 34 & 40 & 35 & 109 \\
Total & & 69 & 77 & 71 & 217 \\
\hline
\end{tabular}

\section{Chi-Square Tests}

\begin{tabular}{|l|r|r|r|}
\hline & Value & df & \multicolumn{1}{c|}{$\begin{array}{c}\text { Asmp. Sig. } \\
\text { (2-sided) }\end{array}$} \\
\hline Fearson Chi-Square & $.141^{a}$ & 2 & .932 \\
Likelihood Ratio & .141 & 2 & .932 \\
N of Valid Cases & 217 & & \\
\hline
\end{tabular}

a. 0 cells (0\%) have expected count less than 5 . The minimum expected count is 34.34 .

Symmetric Measures

\begin{tabular}{|l|r|r|}
\hline & Value & Approx Sig. \\
\hline Nominal by Nominal Contingency Coeficient & .025 & .932 \\
Nof Valid Cases & 217 & \\
\hline
\end{tabular}




\section{$\underline{\mathrm{H}}_{1}$ Conclusions:}

Results of this test are not statistically significant and do not support the hypothesis that salience of business processes identified for measurement correlate to firm value. For this test, the respondent Ranks (rates) from 1 most important to 4 least important process types related to performance measures used, so lower mean ranking in this study indicates higher perceived importance of process types related to performance measures. High performers and low performers rank the processes similarly with priority processes ranking highest, followed by background and then identity processes. Medium performers rank identity processes highest followed by background and then priority processes. Results for the two sub hypotheses $\mathrm{H}_{1 \mathrm{a}}$ and $\mathrm{H}_{1 \mathrm{~b}}$ also are not significant at 0.05 . 
Hypothesis 2 Test:

$\mathrm{H}_{2}$ : Nonfinancial performance measures are more correlated to firm value than

financial measures as tested by Kruskal-Wallis one-way ANOVA.

Hypothesis Test Summary

\begin{tabular}{|llll|}
\hline \multicolumn{1}{|c}{ Null Hypothesis } & \multicolumn{1}{c}{ Test } & Sig. & Decision \\
\hline $1 \begin{array}{l}\text { The distribution of Financial } \\
\text { Measures is the same across } \\
\text { categories of } 1 \mathrm{H}, 2 \mathrm{M}, 3 \mathrm{~L} \text { Rank. }\end{array}$ & $\begin{array}{l}\text { Independent- } \\
\text { Samples } \\
\text { Kruskal- } \\
\text { Wallis Test }\end{array}$ & .077 & $\begin{array}{l}\text { Retain the } \\
\text { null } \\
\text { hypothesis. }\end{array}$ \\
\hline $\begin{array}{l}\text { The distribution of Non-Financial } \\
\text { Measures is the same across } \\
\text { categories of } 1 \mathrm{H}, 2 \mathrm{M}, 3 \mathrm{~L} \text { Rank. }\end{array}$ & $\begin{array}{l}\text { Independent- } \\
\text { Samples } \\
\text { Kruskal- } \\
\text { Wallis Test }\end{array}$ & .004 & $\begin{array}{l}\text { Reject the } \\
\text { null } \\
\text { hypothesis. }\end{array}$ \\
\hline
\end{tabular}

Asymptotic significances are displayed. The significance level is .05 .

\section{Figure 13: $\mathrm{H}_{2}$ Pre-Adjustment Results}

\section{$\underline{\mathrm{H}}_{2} \underline{\text { Adjustments }}$}

$\mathrm{H}_{2}$ Test Data: Delete ID 17 and ID 50 No responses.

$\mathrm{H}_{2}$ Executive: Delete ID 3 Supervisor.

$\mathrm{H}_{2}$ Supervisor: Delete ID 5 Executive.

Include non response from ID 51 for nonfinancial since financial is used. 
TABLE 48

\section{$\mathrm{H}_{2}$ KRUSKAL-WALLIS MEANS}

\begin{tabular}{|l|l|r|r|}
\hline & Low & Medium & High \\
\hline Total Financial & 28.86 & 33.20 & 42.06 \\
\hline Total Nonfinancial & 27.33 & 31.80 & 44.37 \\
\hline & & & \\
\hline & & & \\
\hline Executive Financial & Low & Medium & High \\
\hline Executive Nonfinancial & 28.03 & 33.40 & 41.07 \\
\hline & 26.86 & 31.54 & 43.50 \\
\hline & & & \\
\hline & & & \\
\hline Supervisor Financial & 28.69 & 32.08 & 41.80 \\
\hline Supervisor Nonfinancial & 27.28 & 30.50 & 44.15 \\
\hline
\end{tabular}




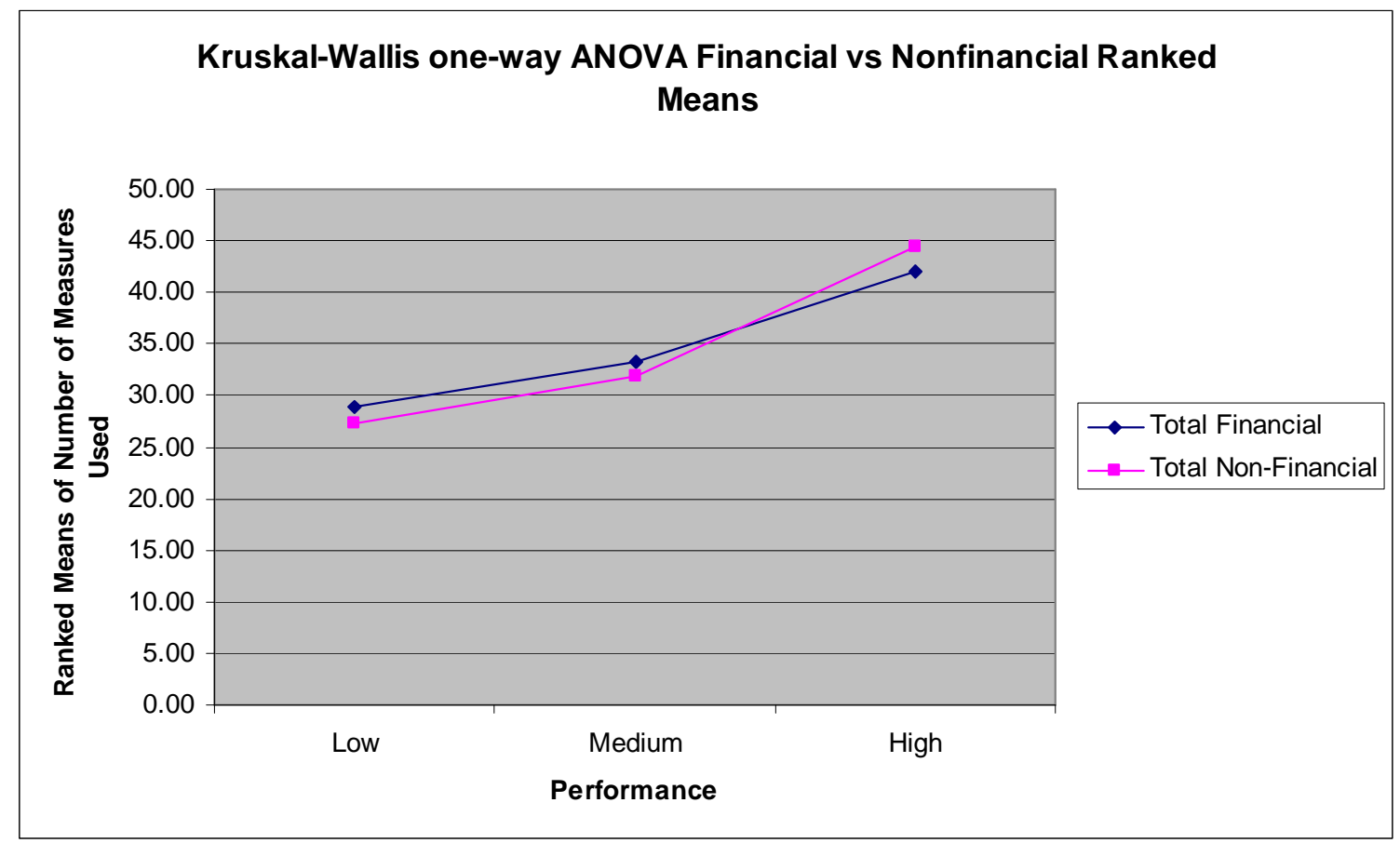

Hypothesis Test Summary

\begin{tabular}{|c|c|c|c|c|}
\hline & Null Hypothesis & Test & Sig. & Decision \\
\hline 1 & $\begin{array}{l}\text { The distribution of Financial } \\
\text { Measures is the same across } \\
\text { categories of } 1 \mathrm{H}, 2 \mathrm{M}, 3 \mathrm{~L} \text { Rank. }\end{array}$ & $\begin{array}{l}\text { Independent- } \\
\text { Samples } \\
\text { Kruskal- } \\
\text { Wallis Test }\end{array}$ & .077 & $\begin{array}{l}\text { Retain the } \\
\text { null } \\
\text { hypothesis }\end{array}$ \\
\hline 2 & $\begin{array}{l}\text { The distribution of Non-Financial } \\
\text { Measures is the same across } \\
\text { categories of } 1 \mathrm{H}, 2 \mathrm{M}, 3 \mathrm{~L} \text { Rank. }\end{array}$ & $\begin{array}{l}\text { Independent- } \\
\text { Samples } \\
\text { Kruskal- } \\
\text { Wallis Test }\end{array}$ & .012 & $\begin{array}{l}\text { Reject the } \\
\text { null } \\
\text { hypothesis }\end{array}$ \\
\hline
\end{tabular}

Asymptotic significances are displayed. The significance level is .05 .

Figure 14: $\mathbf{H}_{2}$ Total Results 


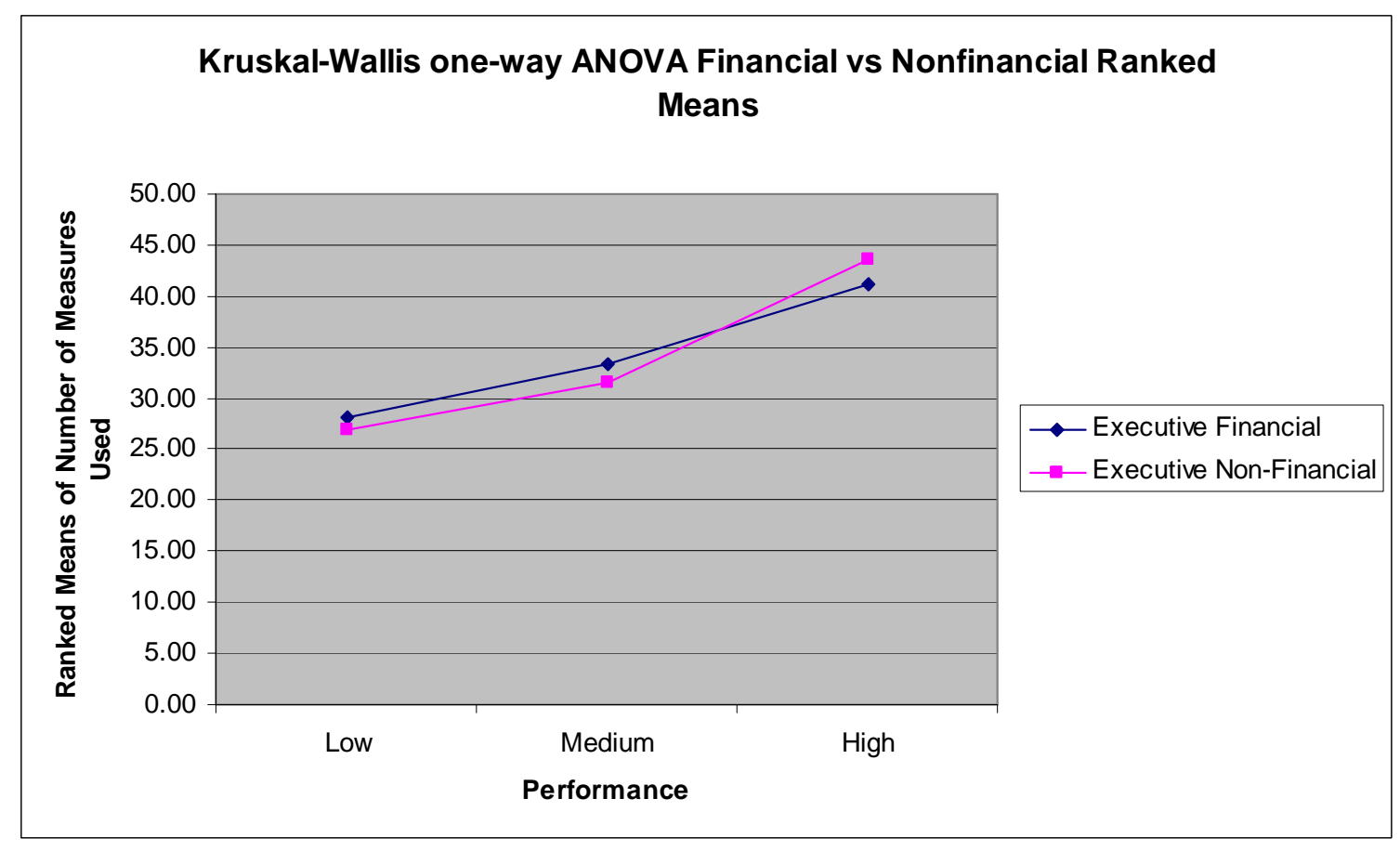

Hypothesis Test Summary

\begin{tabular}{|llll|}
\hline \multicolumn{1}{|c}{ Null Hypothesis } & \multicolumn{1}{c}{ Test } & Sig. & Decision \\
\hline 1 & $\begin{array}{l}\text { The distribution of Financial } \\
\text { Measures is the same across } \\
\text { categories of } 1 \mathrm{H}, 2 \mathrm{M}, 3 \mathrm{~L} \text { Rank. }\end{array}$ & $\begin{array}{l}\text { Independent- } \\
\begin{array}{l}\text { Samples } \\
\text { Kruskal- } \\
\text { Wallis Test }\end{array}\end{array}$ & $.087 \begin{array}{l}\text { Retain the } \\
\text { null } \\
\text { hypothesis. }\end{array}$ \\
\hline $\begin{array}{l}\text { The distribution of Non-Financial } \\
\text { Measures is the same across } \\
\text { categories of } 1 \mathrm{H}, 2 \mathrm{M}, 3 \mathrm{~L} \text { Rank. }\end{array}$ & $\begin{array}{l}\text { Independent- } \\
\text { Samples } \\
\text { Kruskal- } \\
\text { Wallis Test }\end{array}$ & .014 & $\begin{array}{l}\text { Reject the } \\
\text { null } \\
\text { hypothesis. }\end{array}$ \\
\hline
\end{tabular}

Asymptotic significances are displayed. The significance level is .05 .

\section{Figure 15: $\mathrm{H}_{2}$ Executive Results}




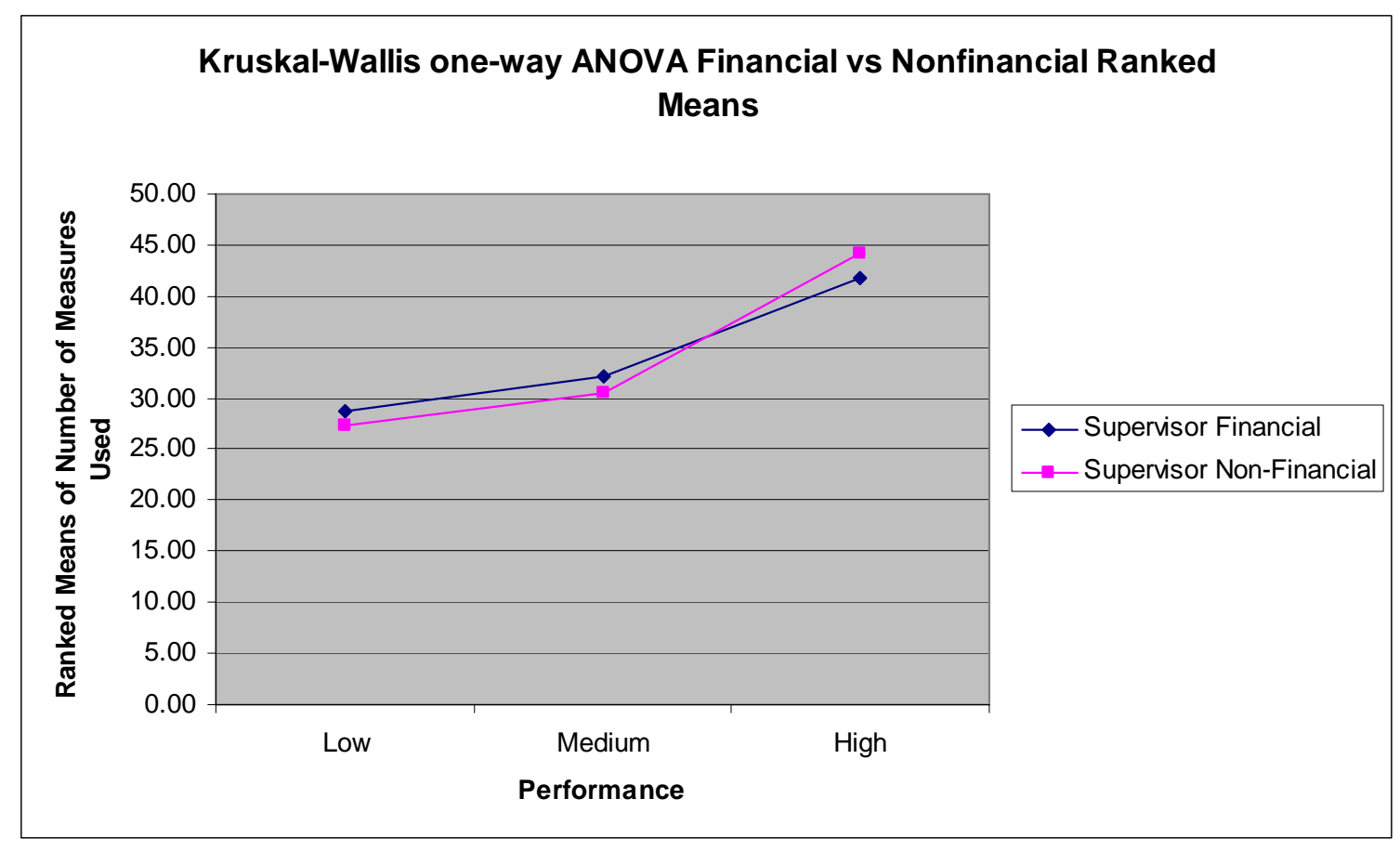

Hypothesis Test Summary

\begin{tabular}{|c|c|c|c|c|}
\hline & Null Hypothesis & Test & Sig. & Decision \\
\hline 1 & $\begin{array}{l}\text { The distribution of Financial } \\
\text { Measures is the same across } \\
\text { categories of } 1 \mathrm{H}, 2 \mathrm{M}, 3 \mathrm{~L} \text { Rank. }\end{array}$ & $\begin{array}{l}\text { Independent- } \\
\text { Samples } \\
\text { Kruskal- } \\
\text { Wallis Test }\end{array}$ & .065 & $\begin{array}{l}\text { Retain the } \\
\text { null } \\
\text { hypothesis. }\end{array}$ \\
\hline 2 & $\begin{array}{l}\text { The distribution of Non-Financial } \\
\text { Measures is the same across } \\
\text { categories of } 1 \mathrm{H}, 2 \mathrm{M}, 3 \mathrm{~L} \text { Rank. }\end{array}$ & $\begin{array}{l}\text { Independent- } \\
\text { Samples } \\
\text { Kruskal- } \\
\text { Wallis Test }\end{array}$ & .009 & $\begin{array}{l}\text { Reject the } \\
\text { null } \\
\text { hypothesis. }\end{array}$ \\
\hline
\end{tabular}

Asymptotic significances are displayed. The significance level is .05 .

\section{Figure 16: $\mathrm{H}_{2}$ Supervisor Results}




\section{$\underline{\mathrm{H}}_{2}$ Conclusions:}

High performers show greatest utilization of both financial and nonfinancial measures, followed by medium performers, with low performers utilizing both measures the least. Nonfinancial performance measures are more correlated to firm value than financial measures with the high performers' mean score for nonfinancial measures being higher than for financial measures. By contrast, medium and low performers exhibit the opposite: higher mean scores for financial measures than for nonfinancial measures. Rejection of the null hypothesis results support the hypothesis that nonfinancial performance measures are more correlated to firm value than financial measures and are statistically significant at 0.05 for nonfinancial measures, at 0.09 for financial measures. 
Hypothesis 3 Test:

$\mathrm{H}_{3}$ : The relative importance of nonfinancial performance measures compared to financial measures using the results from $\mathrm{H}_{2}$ is greater in high clock-speed industries than in low clock-speed industries as tested by Cramer's Phi.

For this test it is first necessary as noted in Chapter IV to calculate the KruskalWallis one-way ANOVA ranked means from $\mathrm{H}_{2}$ then reorganize based on three categories: 1) Product; 2) Process; and 3) Performance Measurement System clockspeed (Appendix P). Cramer's Phi is then calculated to find the strength of association for Performance vs. Financial/Nonfinancial Measurement in High, Medium and Low clockspeeds for each of the 3 categories. This test is performed on Total Results (Appendix Q) as summarized on Table 49:

TABLE 49

\section{$\mathrm{H}_{3}$ CRAMER'S PHI BY CLOCKSPEED}

\begin{tabular}{|c|r|r|r|}
\hline \multicolumn{4}{|c|}{ Chi-s quare \& Cramers Phi (V) } \\
\hline & & & $\begin{array}{l}\text { Performance } \\
\text { Measurement } \\
\text { System }\end{array}$ \\
Clockspeed & Product & Process & 0.16 \\
\hline High $\varphi c$ & 0.10 & 0.06 & 0.812 \\
\hline High X2 & 0.424 & 0.184 & 0.19 \\
\hline Medium $\varphi c$ & 0.31 & 0.24 & 1.024 \\
\hline Medium X2 & 1.227 & 1.027 & 0.53 \\
\hline Low $\varphi c$ & 0.51 & \#DIV0! & 1.404 \\
\hline Low X2 & 2.339 & \#DIV0! & 0.05 \\
\hline \multicolumn{4}{|c|}{ Retain the null hypothesis } \\
\hline Significance & df $=2$ & 0.1 & 5.991 \\
\hline Required X2 & \multicolumn{4}{|c|}{4.605} & \\
\hline
\end{tabular}


H3 is not supported by the evidence. Though lacking statistical significance at 0.05 , greater strength of association for Performance vs. Financial/Nonfinancial Measurement is exhibited in low clockspeed systems identified by respondents than in high clockspeed systems.

Hypothesis 4 Test:

$\mathrm{H}_{4}$ : The number of categories of Balanced Scorecard metrics used correlate to firm value as tested by Kruskal-Wallis one-way ANOVA.

Hypothesis Test Summary

\begin{tabular}{|c|c|c|c|c|}
\hline & Null Hypothesis & Test & Sig. & Decision \\
\hline & $\begin{array}{l}\text { The distribution of BSC Used is the } \\
\text { same across categories of } 1 \mathrm{H}, 2 \mathrm{M} \text {, } \\
3 \mathrm{~L} \text { Rank. }\end{array}$ & $\begin{array}{l}\text { Independent- } \\
\text { Samples } \\
\text { Kruskal- } \\
\text { Wallis Test }\end{array}$ & .009 & $\begin{array}{l}\text { Reject the } \\
\text { null } \\
\text { hypothesis. }\end{array}$ \\
\hline
\end{tabular}

Asymptotic significances are displayed. The significance level is .05 .

\section{Figure 17: $\mathrm{H}_{4}$ Pre-Adjustment Results}

\section{$\underline{\mathrm{H}}_{4}$ Adjustments}

H4 Test Data: Delete ID 17 and ID 50 no responses.

H4 Executive: Delete ID 3 Supervisor.

H4 Supervisor: Delete ID 5 Executive. 
Responses for this test are summarized in Appendix R. Of the nine companies using less than the full four categories of measurement (eleven companies including the two non-response companies), a disproportionate percentage are low performers as can be seen in Table 50:

TABLE 50

BALANCED SCORECARD CATEGORY UTILIZATION

\begin{tabular}{|l|l|l|l|r|}
\hline & $\begin{array}{l}\text { Total } \\
\text { Reporting } \\
\text { Respondents }\end{array}$ & $\begin{array}{l}\text { Respondents } \\
\text { Reporting }<4 \\
\text { Categories }\end{array}$ & $\begin{array}{l}\text { \% of Respondents } \\
\text { by Performance Category } \\
\text { Reporting <4 Categories }\end{array}$ & $\begin{array}{l}\text { \% of Respondents } \\
\text { Reporting }<4 \\
\text { Categories }\end{array}$ \\
\hline High & 27 & 1 & $4 \%$ & $11 \%$ \\
\hline Medium & 25 & 3 & $12 \%$ & $33 \%$ \\
\hline Low & 20 & 5 & $25 \%$ & $56 \%$ \\
\hline
\end{tabular}

\begin{tabular}{|c|c|c|c|c|}
\hline \multicolumn{4}{|c|}{ Balanced Scorecard } & \\
\hline Categories Used & \multicolumn{3}{|c|}{ Performance } & \\
\hline & Low & Medium & High & \\
\hline 4 & 13 & 22 & 26 & \\
\hline 3 & 3 & 2 & 1 & \\
\hline 2 & 1 & 1 & & \\
\hline 1 & 1 & & & $p=0.06$ \\
\hline 0 & 2 & & & $p=0.009$ \\
\hline Total & 20 & 25 & 27 & \\
\hline
\end{tabular}

TABLE 51

\section{BALANCED SCORECARD KRUSKAL-WALLIS MEANS}

\begin{tabular}{|l|r|r|r|}
\hline & \multicolumn{1}{|l|}{ Low } & \multicolumn{1}{l|}{ Medium } & High \\
\hline Mean Scores & 30.17 & 35.82 & 38.76 \\
\hline Executive & 29.81 & 35.21 & 38.28 \\
\hline Supervisor & 29.81 & 35.21 & 38.28 \\
\hline
\end{tabular}




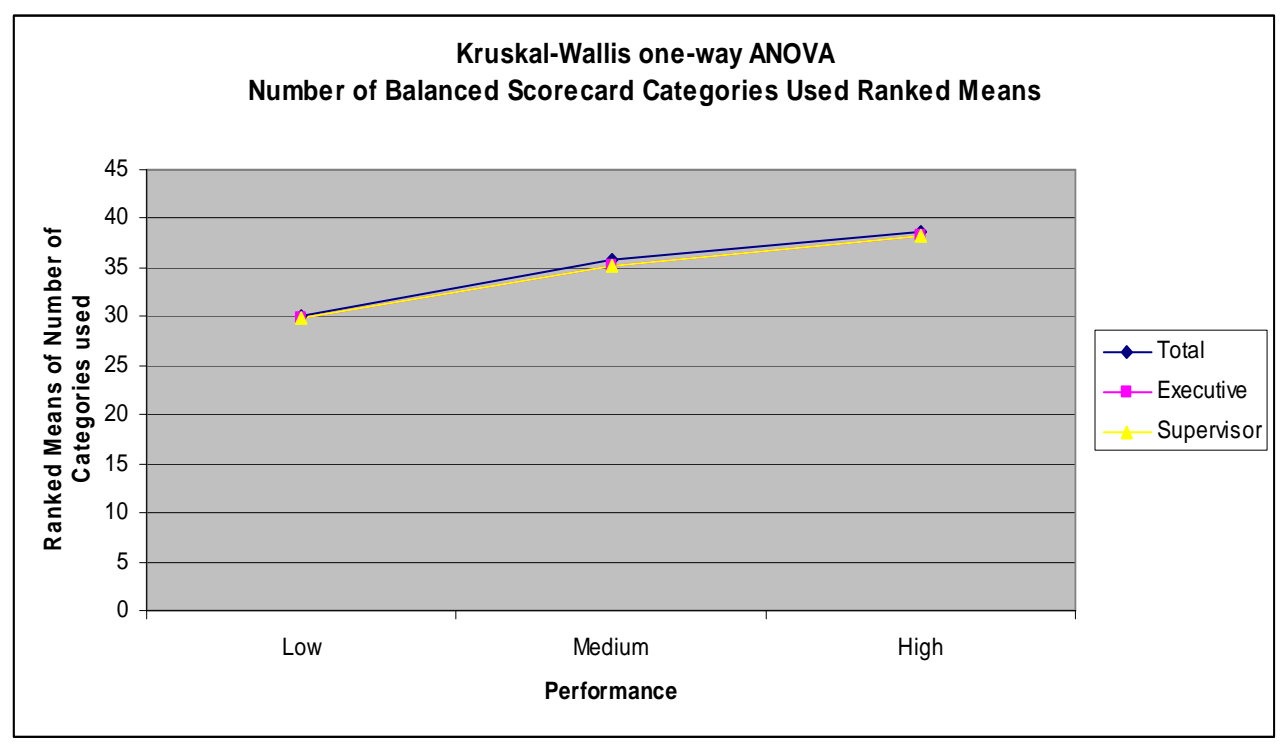

Hypothesis Test Summary

\begin{tabular}{|llrl|}
\hline \multicolumn{1}{|c|}{ Null Hypothesis } & \multicolumn{1}{c|}{ Test } & Sig. & Decision \\
\hline $\begin{array}{l}\text { The distribution of BSC Used is the } \\
\text { same across categories of } 1 \mathrm{H}, 2 \mathrm{M}, \\
\text { 3L Rank. }\end{array}$ & $\begin{array}{l}\text { Independent- } \\
\text { Samples } \\
\begin{array}{l}\text { Kruskal- } \\
\text { Wallis Test }\end{array}\end{array}$ & .057 & $\begin{array}{l}\text { Reject the } \\
\text { null } \\
\text { hypothesis. }\end{array}$ \\
\hline
\end{tabular}

Asymptotic significances are displayed. The significance level is .06.

Figure 18: $\mathrm{H}_{4}$ Total Results

Hypothesis Test Summary

\begin{tabular}{|llrl|}
\hline \multicolumn{1}{|c|}{ Null Hypothesis } & \multicolumn{1}{c|}{ Test } & Sig. & Decision \\
\hline & $\begin{array}{l}\text { The distribution of BSC Used is the } \\
\text { same across categories of } 1 \mathrm{H}, 2 \mathrm{M} \text {, } \\
\text { 3L Rank. }\end{array}$ & $\begin{array}{l}\text { Independent- } \\
\text { Samples } \\
\text { Kruskal- } \\
\text { Wallis Test }\end{array}$ & $.059 \begin{array}{l}\text { Reject the } \\
\text { null } \\
\text { hypothesis. }\end{array}$ \\
\hline
\end{tabular}

Asymptotic significances are displayed. The significance level is .06 .

\section{Figure 19: $\mathrm{H}_{4}$ Executive \& Supervisor Results}




\section{$\underline{\mathrm{H}}_{4}$ Conclusions:}

Rejection of the null hypothesis results of this test support the hypothesis that the number of categories of balanced scorecard metrics used correlate to firm value at a 0.06 level of significance. The high level of significance in the PreAdjustment Results is due to the inclusion of non-responders ID 17 and ID 50 who are both low performers. The results of this test would support the hypothesis at a high level of significance with the assumption that non-usage of any category of balanced scorecard by these two respondents is valid. 


\section{CHAPTER VI}

\section{DISCUSSION}

This chapter presents: 1) Discussion of results and implications of the research;

2) Limitations of the research; 3) Strengths of the research; then 4) Suggestions for further research.

\section{$\underline{\text { Results and Implications }}$}

\section{$\underline{\mathrm{H}}_{1}$ : Salience of Business Process}

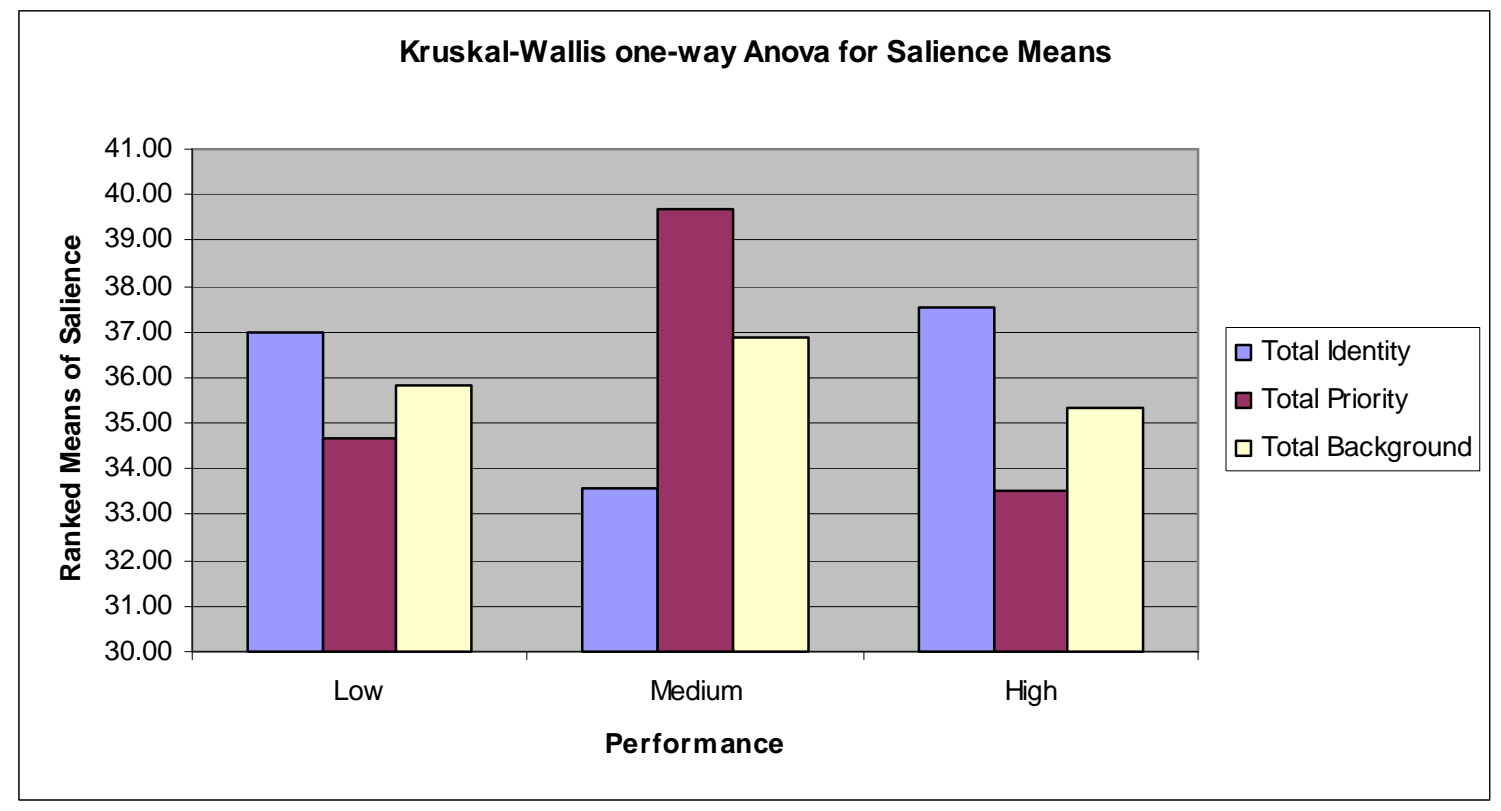

Figure 20: Process Salience Usage by Performance

The rankings in this test are rated based on 1 most important to 4 least important process types related to performance measures used, so lower mean ranking in this study indicates higher perceived importance of process types related to performance measures. 
It is hypothesized that: 1) High performers prioritize identity processes as most important, followed by priority processes then background processes; 2) Low performers prioritize background processes as most important, followed by priority processes then identity processes; and 3) Medium performers fall somewhere in the middle of the two extremes. The pattern uncovered, though not statistically significant, is that high performers and low performers rank similarly with greatest importance placed on priority processes followed by background processes then identity processes, while medium performers place most importance on identity processes followed by background then priority processes. High, medium and low performers all rate background processes in the middle of their importance rankings. In addition to the lack of statistical significance, the following section on limitations notes that reliability of this specific test also is not high. Given the lack of statistical significance and reliability valid inference cannot be made from the results of this test.

\section{$\underline{\mathrm{H}}_{2}$ : Financial vs. Nonfinancial Performance Measures}

A number of studies, including Chow and Van der Stede (2006), suggest that nonfinancial measures are better than financial measures for improving performance because financial measures are lagging indicators, but “...little empirical evidence is available on the relation between nonfinancial measures and financial performance" (Banker, Potter \& Srinivasan, 2000, p. 65). Ittner and Larker (2003) find that few companies realize the benefits of nonfinancial performance measures because they: 1) Don't link measures to strategy; 2) Don't validate the links between nonfinancial 
performance measures and future financial results; 3) Don't set the right performance targets; or 4) Employ metrics that lack statistical validity. They further note that "outstanding" nonfinancial performance often produces diminishing or even negative economic returns (p. 5).

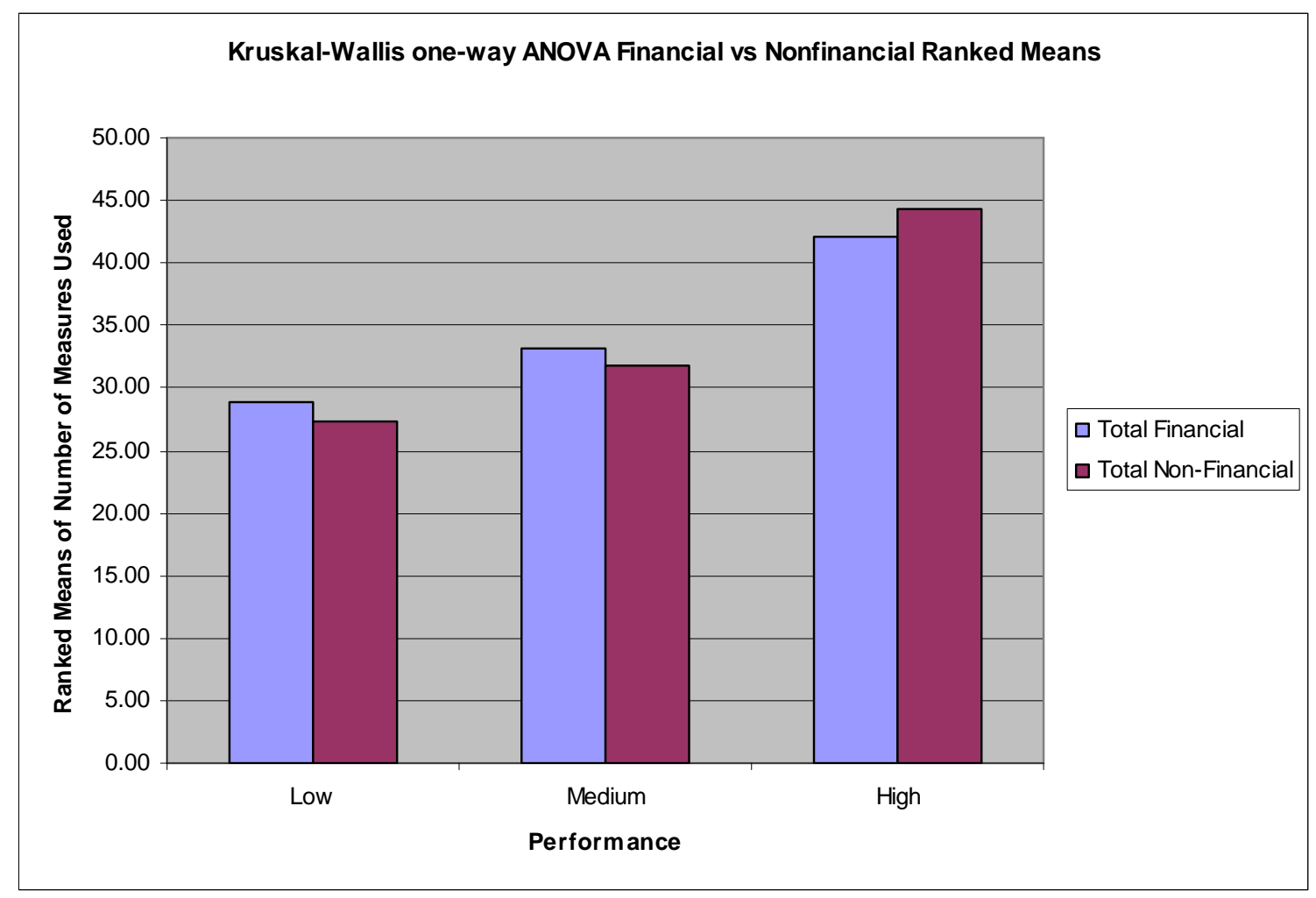

Figure 21: Ranked Means of Financial and Nonfinancial Measures Used by Performance

This study provides statistically significant empirical evidence that high performing companies rank higher in utilization of nonfinancial measures than do medium or low performing companies. This result is obtained blind to the specific measures used, linkage between measures and strategy, or statistical validity of the measures used. For practitioners it can be inferred that benefit is derived from greater 
usage of both financial and nonfinancial measures, with the greatest benefit derived from use of nonfinancial measures. For researchers, further study regarding specific measurements, statistical validity of the measurements, and linkages between selected measurements and strategy may provide value.

\section{$\underline{\mathrm{H}}_{3}:$ Financial / Nonfinancial Measures Relative to Clockspeed}

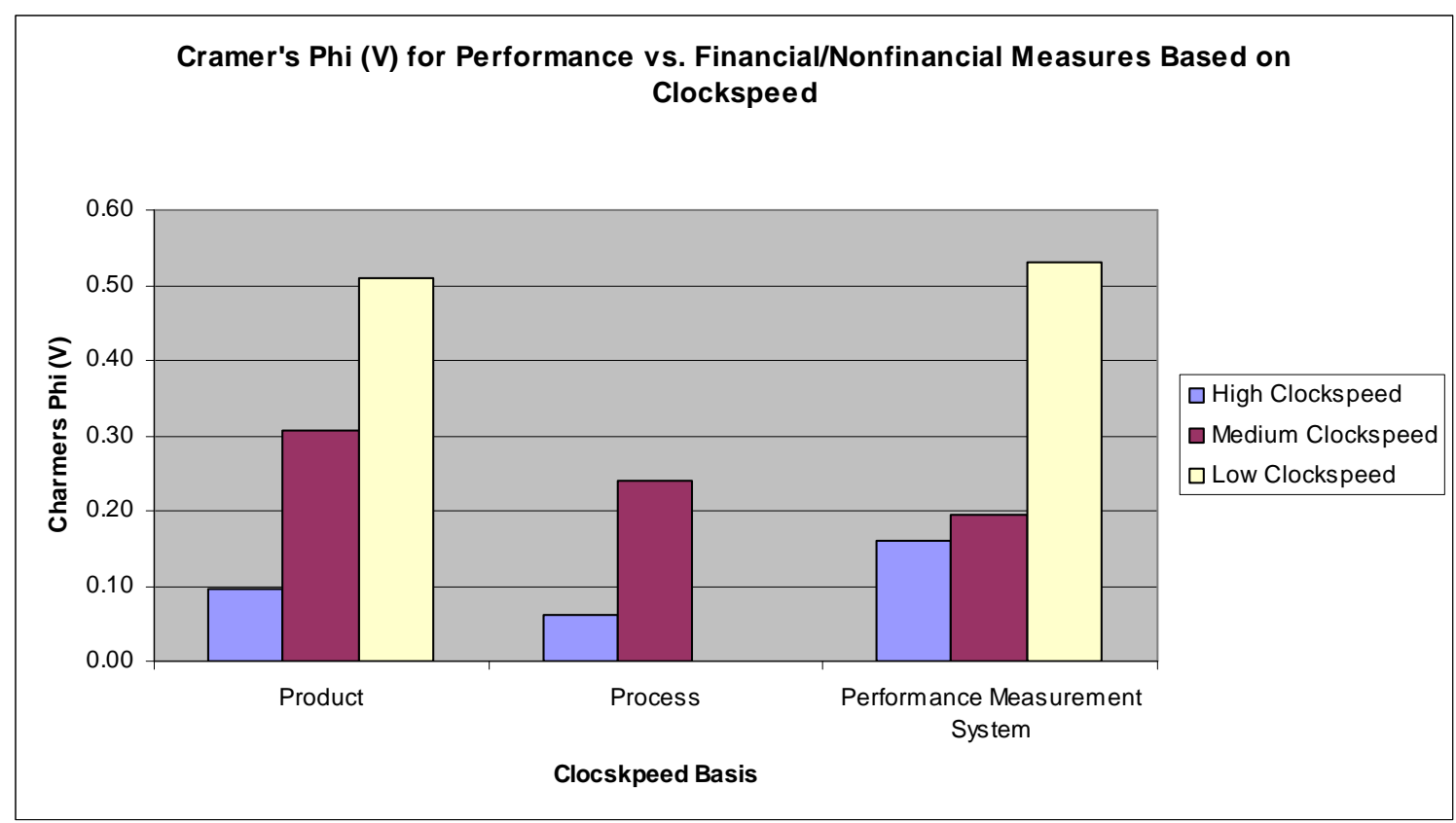

Figure 22: Cramer's Phi (V) Based on Clockspeed

It is hypothesized that the relative importance of nonfinancial performance measures compared to Financial measures using the results from $\mathrm{H}_{2}$ is greater in high clockspeed industries than in low clockspeed industries as tested by Cramer's Phi based on the logic that nonfinancial performance measures result in shorter lag periods of feedback than financial performance measures, and the relative importance of the lag time will be heightened in higher clockspeed industries as defined by Fine (1998); with 
higher clockspeed industries being more susceptible to dynamic archetypes associated with delayed feedback (Senge,1990; Sterman, 2000). Cramer's Phi (V) values in this test are counter to that anticipated with greater strengths of association for Performance vs. Financial/Nonfinancial Measurement exhibited in low clockspeed industries than in high clockspeed industries. In all cases, however, $\chi^{2}$ is far below the 5.991 required for statistical significance at a 0.05 level with 2 degrees of freedom (Appendix Q). Due to the lack of statistical significance valid inference cannot be made from the results of this test.

\section{$\underline{\mathrm{H}}_{4}:$ Balanced Scorecard Category Metrics Used}

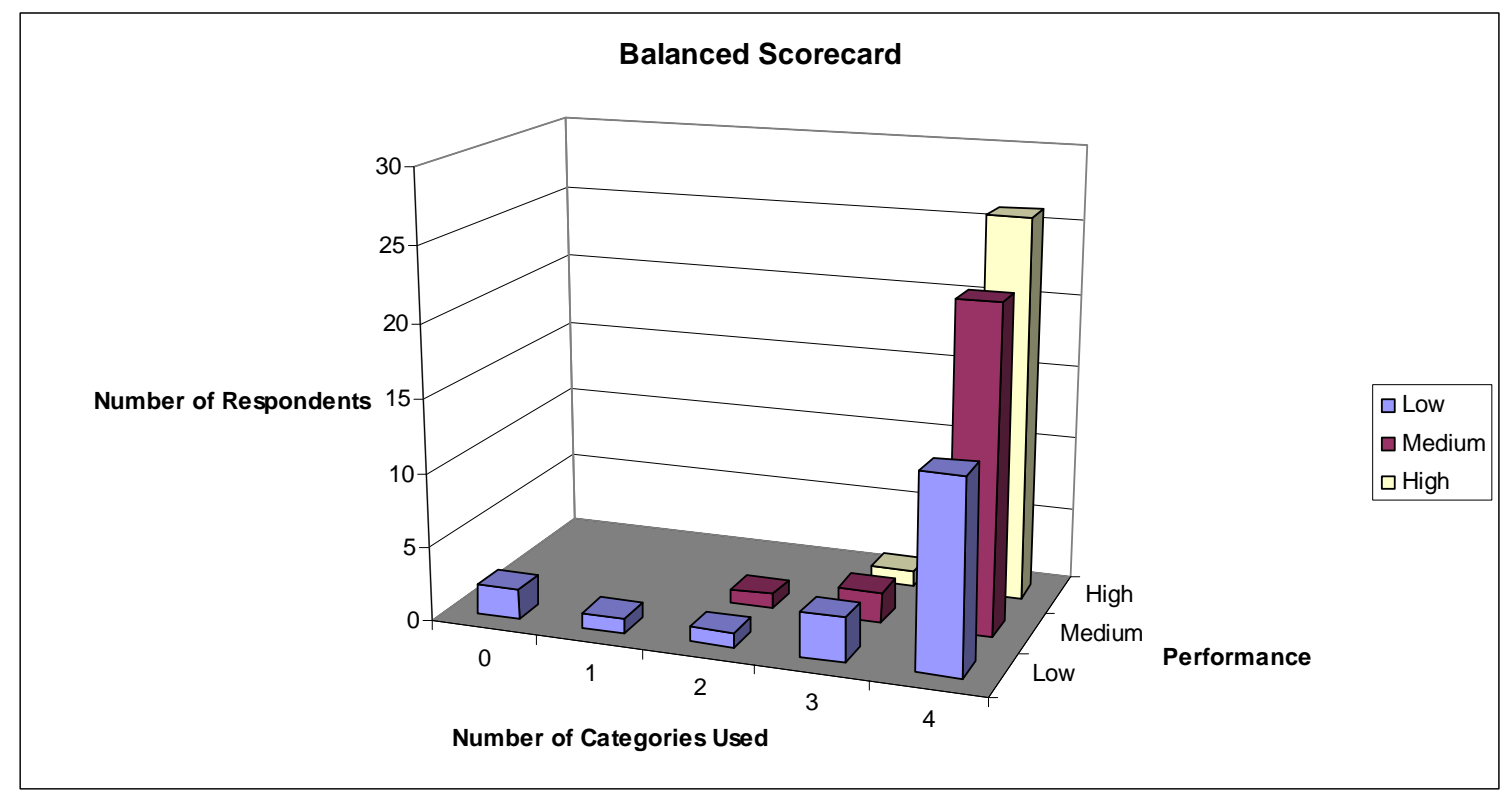

Figure 23: Balanced Scorecard Utilization by Performance Category

Figure 23 highlights the decreasing utilization of the full spectrum of balanced scorecard category metrics by medium and low performing companies relative to high 
performing companies in this test. Going from high performers to low performers the number of respondents decreases while the number of companies reporting usage of fewer that the full 4 categories available increases. It also demonstrates that of the companies using fewer than the full four possible balanced scorecard category metrics, the high performing company reports using 3 of the 4 categories while the low performing companies report as few as 1 category used. As noted in Chapter V, two low performing companies also report no measurements from any of the categories available, inclusion of which would have resulted in a 0.009 level of significance.

Osama (2006) finds only anecdotal improvements based on implementation of individual elements of the Balanced Scorecard (p. ix). Neelly, Kennerley and Martinez (2004) note a paucity of empirical evidence exploring the impact of balanced scorecard and suggest a need for further research into the performance impact of balanced scorecards. Ittner and Larker (2003), while commenting on the lack of benefits derived from using nonfinancial measures note middle managers perceive the balanced scorecard as a "four bucket" or "smorgasbord" approach because the four perspectives are imposed by upper management regardless of the business unit's strategy or objectives, and Bourne, Kennerley and Franco-Santos (2005) find that high performing business unit managers simply use the scorecard data to check their own assumptions.

This study provides statistically significant (0.06 level) empirical evidence that utilization of performance measures across the four categories of balanced scorecard metrics correlates to higher performance. This result is observed with no information 
regarding implementation of the balanced scorecard methodology. For practitioners it can be inferred that benefit is derived from defining and using measurement metrics from each of the four categories. For researchers, further study regarding implementation relative to business units' strategy and objectives may provide value.

\section{$\underline{\text { Limitations }}$}

Ittner, Larker and Meyer (2003) note the issue of weights placed by managers on the importance of measures that are used. Weighting in this study is based on the number of measures defined by the respondents, so there is no assurance that the "number of measures" in any category defined by respondents reflects the weight placed on the value of those measures by managers for decision making. In addition, the dependent variable is based on stock price so non-publicly traded companies are not represented in the test.

Potential for bias exists in the test due to insufficient: Construct Validity; Internal Validity; External Validity; and/or Reliability. Each is addressed relative to the countermeasures that are proposed in Chapter IV.

\section{Construct Validity:}

Construct Validity asks whether what is measured is what is wanted to be measured. "The major threats to construct validity are those created by bias either 
through the process of observing itself, or bias introduced by the observation method" (Atkinson \& Shafir, 1998, p. 60).

The potential for introduction of bias by the observation method or process of observing is mitigated with the Survey Consent Form (Appendix S), that identifies the subject matter of the research, the mode of observation, and the background training taken into the study.

Internal Validity:

Internal Validity: “... asks whether the researcher has taken steps to ensure that the evidence used to infer a casual [sic] relationship is complete. That is... [to] avoid reporting a spurious correlation as causal" (Atkinson \& Shafir, 1998, p. 61).

The theoretic basis of the proposed correlation is defined in Chapter III -Description of the Problem. Tests for correlation between different variables have been performed to confirm that there is little chance that test results are spurious correlation. Non-scale variables are tested for correlation using Kruskal-Wallis one-way ANOVA, scale relationships are tested for Bilateral Correlation. 
Performance relative to: Clockspeed (Product Change, Process Change, and Perf.

Measure Change); Asset Size; and Age are analyzed. None test significant for correlation with Kruskal-Wallis one-way ANOVA as shown in Figure 24:

Hypothesis Test Summary

\begin{tabular}{|c|c|c|c|c|}
\hline & Null Hypothesis & Test & Sig. & Decision \\
\hline 1 & $\begin{array}{l}\text { The distribution of Product Change } \\
(1,2,3) \text { is the same across } \\
\text { categories of } 1 \mathrm{H}, 2 \mathrm{M}, 3 \mathrm{~L} \text { Rank. }\end{array}$ & $\begin{array}{l}\text { Independent- } \\
\text { Samples } \\
\text { Kruskal- } \\
\text { Wallis Test }\end{array}$ & .966 & $\begin{array}{l}\text { Retain the } \\
\text { null } \\
\text { hypothesis. }\end{array}$ \\
\hline 2 & $\begin{array}{l}\text { The distribution of Process Change } \\
\text { is the same across categories of } \\
1 \mathrm{H}, 2 \mathrm{M}, 3 \mathrm{~L} \text { Rank. }\end{array}$ & $\begin{array}{l}\text { Independent- } \\
\text { Samples } \\
\text { Kruskal- } \\
\text { Wallis Test }\end{array}$ & .300 & $\begin{array}{l}\text { Retain the } \\
\text { null } \\
\text { hypothesis. }\end{array}$ \\
\hline 3 & $\begin{array}{l}\text { The distribution of Perf. Measure } \\
\text { Change is the same across } \\
\text { categories of } 1 \mathrm{H}, 2 \mathrm{M}, 3 \mathrm{~L} \text { Rank. }\end{array}$ & $\begin{array}{l}\text { Independent- } \\
\text { Samples } \\
\text { Kruskal- } \\
\text { Wallis Test }\end{array}$ & .452 & $\begin{array}{l}\text { Retain the } \\
\text { null } \\
\text { hypothesis. }\end{array}$ \\
\hline 4 & $\begin{array}{l}\text { The distribution of Asset Size } \\
\text { (thousands) is the same across } \\
\text { categories of } 1 \mathrm{H}, 2 \mathrm{M}, 3 \mathrm{~L} \text { Rank. }\end{array}$ & $\begin{array}{l}\text { Independent- } \\
\text { Samples } \\
\text { Kruskal- } \\
\text { Wallis Test }\end{array}$ & .570 & $\begin{array}{l}\text { Retain the } \\
\text { null } \\
\text { hypothesis. }\end{array}$ \\
\hline 5 & $\begin{array}{l}\text { The distribution of Age (yrs as of } \\
12 / 08 \text { ) is the same across } \\
\text { categories of } 1 \mathrm{H}, 2 \mathrm{M}, 3 \mathrm{~L} \text { Rank. }\end{array}$ & $\begin{array}{l}\text { Independent- } \\
\text { Samples } \\
\text { Kruskal- } \\
\text { Wallis Test }\end{array}$ & .172 & $\begin{array}{l}\text { Retain the } \\
\text { null } \\
\text { hypothesis. }\end{array}$ \\
\hline
\end{tabular}

Asymptotic significances are displayed. The significance level is .05 .

\section{Figure 24: Performance Relative to Clockspeed, Asset Size and Age}


Position relative to: Financial Measures; Nonfinancial Measures; Balanced

Scorecard Categories used (BSC); Identity Process rank; Priority Process Rank; and

Background Process Rank are analyzed. None test significant for correlation with

Kruskal-Wallis one-way ANOVA as shown in Figures 25:

Hypothesis Test Summary

\begin{tabular}{|c|c|c|c|c|}
\hline & Null Hypothesis & Test & Sig. & Decision \\
\hline 1 & $\begin{array}{l}\text { The distribution of Financial } \\
\text { Measures is the same across } \\
\text { categories of Position. }\end{array}$ & $\begin{array}{l}\text { Independent- } \\
\text { Samples } \\
\text { Kruskal- } \\
\text { Wallis Test }\end{array}$ & .199 & $\begin{array}{l}\text { Retain the } \\
\text { null } \\
\text { hypothesis. }\end{array}$ \\
\hline 2 & $\begin{array}{l}\text { The distribution of Non-Financial } \\
\text { Measures is the same across } \\
\text { categories of Position. }\end{array}$ & $\begin{array}{l}\text { Independent- } \\
\text { Samples } \\
\text { Kruskal- } \\
\text { Wallis Test }\end{array}$ & .410 & $\begin{array}{l}\text { Retain the } \\
\text { null } \\
\text { hypothesis. }\end{array}$ \\
\hline 3 & $\begin{array}{l}\text { The distribution of BSC Used is the } \\
\text { same across categories of Position. }\end{array}$ & $\begin{array}{l}\text { Independent- } \\
\text { Samples } \\
\text { Kruskal- } \\
\text { Wallis Test }\end{array}$ & .909 & $\begin{array}{l}\text { Retain the } \\
\text { null } \\
\text { hypothesis. }\end{array}$ \\
\hline 4 & $\begin{array}{l}\text { The distribution of Identity Process } \\
\text { Rank is the same across } \\
\text { categories of Position. }\end{array}$ & $\begin{array}{l}\text { Independent- } \\
\text { Samples } \\
\text { Kruskal- } \\
\text { Wallis Test }\end{array}$ & .858 & $\begin{array}{l}\text { Retain the } \\
\text { null } \\
\text { hypothesis. }\end{array}$ \\
\hline 5 & $\begin{array}{l}\text { The distribution of Priority Process } \\
\text { Rank is the same across } \\
\text { categories of Position. }\end{array}$ & $\begin{array}{l}\text { Independent- } \\
\text { Samples } \\
\text { Kruskal- } \\
\text { Wallis Test }\end{array}$ & .611 & $\begin{array}{l}\text { Retain the } \\
\text { null } \\
\text { hypothesis. }\end{array}$ \\
\hline 6 & $\begin{array}{l}\text { The distribution of Background } \\
\text { Process Rank is the same across } \\
\text { categories of Position. }\end{array}$ & $\begin{array}{l}\text { Independent- } \\
\text { Samples } \\
\text { Kruskal- } \\
\text { Wallis Test }\end{array}$ & .460 & $\begin{array}{l}\text { Retain the } \\
\text { null } \\
\text { hypothesis. }\end{array}$ \\
\hline
\end{tabular}

Asymptotic significances are displayed. The significance level is .05.

\section{Figure 25: Correlations Relative to Position}


Department relative to: Financial Measures; Nonfinancial Measures; Balanced

Scorecard Categories used; Identity Process rank; Priority Process Rank; and Background

Process Rank are analyzed. None test significant for correlation with Kruskal-Wallis

one-way ANOVA as shown in Figures 26:

Hypothesis Test Summary

\begin{tabular}{|c|c|c|c|c|}
\hline & Null Hypothesis & Test & Sig. & Decision \\
\hline 1 & $\begin{array}{l}\text { The distribution of Financial } \\
\text { Measures is the same across } \\
\text { categories of Deparment. }\end{array}$ & $\begin{array}{l}\text { Independent- } \\
\text { Samples } \\
\text { Kruskal- } \\
\text { Wallis Test }\end{array}$ & .485 & $\begin{array}{l}\text { Retain the } \\
\text { null } \\
\text { hypothesis. }\end{array}$ \\
\hline 2 & $\begin{array}{l}\text { The distribution of Non-Financial } \\
\text { Measures is the same across } \\
\text { categories of Deparment. }\end{array}$ & $\begin{array}{l}\text { Independent- } \\
\text { Samples } \\
\text { Kruskal- } \\
\text { Wallis Test }\end{array}$ & .462 & $\begin{array}{l}\text { Retain the } \\
\text { null } \\
\text { hypothesis. }\end{array}$ \\
\hline 3 & $\begin{array}{l}\text { The distribution of BSC Used is the } \\
\text { same across categories of } \\
\text { Deparment. }\end{array}$ & $\begin{array}{l}\text { Independent- } \\
\text { Samples } \\
\text { Kruskal- } \\
\text { Wallis Test }\end{array}$ & .142 & $\begin{array}{l}\text { Retain the } \\
\text { null } \\
\text { hypothesis. }\end{array}$ \\
\hline 4 & $\begin{array}{l}\text { The distribution of Identity Process } \\
\text { Rank is the same across } \\
\text { categories of Deparment. }\end{array}$ & $\begin{array}{l}\text { Independent- } \\
\text { Samples } \\
\text { Kruskal- } \\
\text { Wallis Test }\end{array}$ & .253 & $\begin{array}{l}\text { Retain the } \\
\text { null } \\
\text { hypothesis. }\end{array}$ \\
\hline 5 & $\begin{array}{l}\text { The distribution of Priority Process } \\
\text { Rank is the same across } \\
\text { categories of Deparment. }\end{array}$ & $\begin{array}{l}\text { Independent- } \\
\text { Samples } \\
\text { Kruskal- } \\
\text { Wallis Test }\end{array}$ & .666 & $\begin{array}{l}\text { Retain the } \\
\text { null } \\
\text { hypothesis. }\end{array}$ \\
\hline 6 & $\begin{array}{l}\text { The distribution of Background } \\
\text { Process Rank is the same across } \\
\text { categories of Deparment. }\end{array}$ & $\begin{array}{l}\text { Independent- } \\
\text { Samples } \\
\text { Kruskal- } \\
\text { Wallis Test }\end{array}$ & .154 & $\begin{array}{l}\text { Retain the } \\
\text { null } \\
\text { hypothesis. }\end{array}$ \\
\hline
\end{tabular}

Asymptotic significances are displayed. The significance level is .05 .

\section{Figure 26: Correlations Relative to Department}


Bilateral Correlation between: Financial Measures; Nonfinancial Measures;

Clockspeed (Product Change, Process Change, and Perf. Measure Change); Balanced Scorecard Categories used (BSC Used); Asset Size; and Age shown in Table 52: 
TABLE 52

BILATERAL CORRELATIONS

\begin{tabular}{|c|c|c|c|c|c|c|c|c|}
\hline 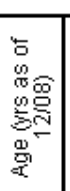 & 管是足 & 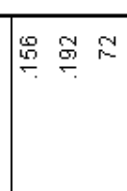 & 9 & 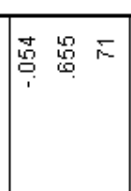 & 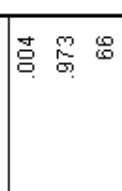 & 莫要视 & 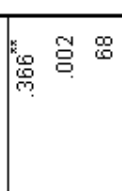 & $-\approx$ \\
\hline 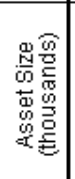 & 总息昌 & $\approx \approx$ & 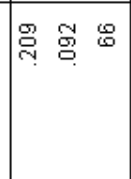 & 管骂器 & 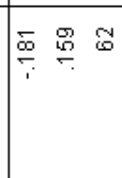 & 骂 哭 & $-\quad \nsubseteq$ & 罯 \\
\hline 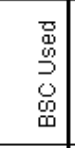 & 悉喜 & 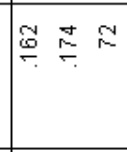 & 开总员 & 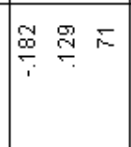 & 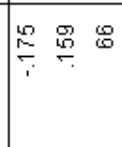 & $-N$ & 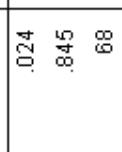 & 吉要品 \\
\hline 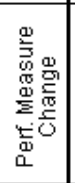 & $\frac{9}{7}$ & $\approx 8$ & 20 & 98 & $-\quad 9$ & 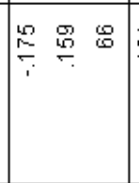 & 总。 & 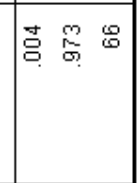 \\
\hline \begin{tabular}{ll|l} 
& \\
\\
0
\end{tabular} & 乎昌 & 㱍 & : 合罾 & $-\quad F$ & 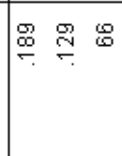 & 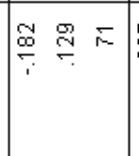 & 器器占 & 吉员不 \\
\hline 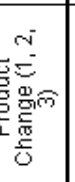 & 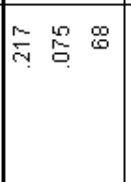 & $\approx \equiv 2$ & $-\quad P$ & 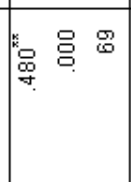 & 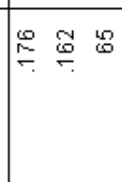 & 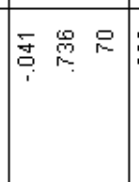 & 密 & $=g$ \\
\hline 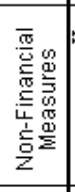 & 吕 & $-\approx$ & $\underset{F}{\oplus} \rightleftharpoons$ & 兽总 & 웡 & $\mathcal{O S O}$ & ㄱำ & 果 \\
\hline 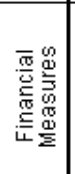 & $-\quad P$ & 佥员 & 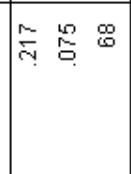 & 乎象 & 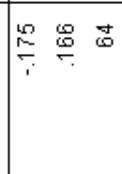 & 曾憘口 & 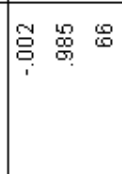 & $=9$ \\
\hline & 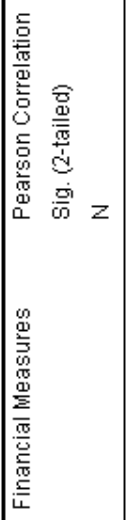 & 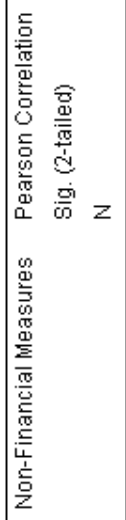 & 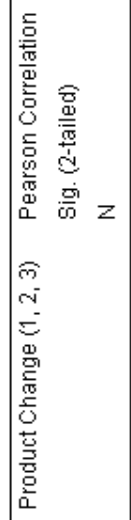 & 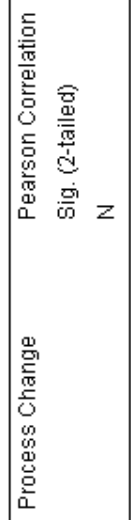 & 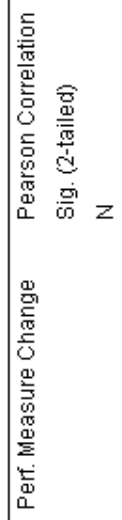 & 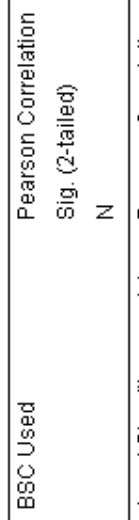 & 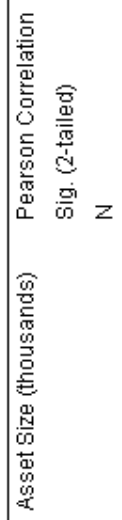 & 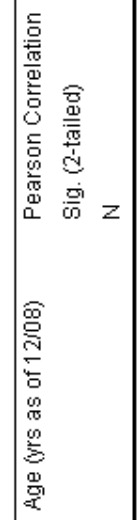 \\
\hline
\end{tabular}


Significant correlations are noted as follows:

Nonfinancial Measures relative to Financial Measures with a Pearson's $r$ of 0.867. Though this correlation is significant at the 0.01 level, similarities and differences in utilization of the two types of measurement based on level of performance are documented in $\mathrm{H}_{2}$.

Process Change rate relative to Product Change rate (clockspeed) with a Pearson's $r$ of 0.480 at a 0.01 level. Correlation between these two is logical, significant, and suggested by Carillo (2005) and Mendelson and Pillai (1999), but has no bearing on hypothesis tests that were performed.

Performance Measure Change rate relative to Nonfinancial Measures with a Pearson's $r$ of -0.272 . Though significant at the 0.05 level, this is not a strong correlation and does not have a causal relationship to any of the hypothesis tests that are performed.

\section{External Validity:}

External Validity: Asks whether the population is clearly identified in order to apply the testing results (Atkinson \& Shafir, 1998, p. 61). 
The definition of the subject population and the means of selection are defined in Chapter IV -- Methods and Techniques. The sample solicited by email to participate in the web based survey is large and representative: 1) Sample is drawn from $100 \%$ of active publicly traded companies downloaded from Mergent Online in 3 Digit NAICS categories; 2) A random number generator is used to define the start number for each 3 digit NAICS Category; 3) A systematic selection of every odd or even numbered company depending on random number for each NAICS category for a $50 \%$ sample (3,578 companies total) is used; and 4) All companies traded $\geq 5$ yrs with email address available (1,732 companies total) in the $50 \%$ sampling are used.

\section{Reliability:}

Reliability: “...asks whether the research can be replicated with the same results" (Atkinson \& Shafir, 1998, p. 62). This research encompasses a large number of industries with results standardized by industry (NAICS Code) over the same time period (end date of financial comparison is defined and identical for all subjects).

The following methods are proposed in Chapter IV to test reliability of the survey instrument: 1) Testing Pilot survey questionnaire, 2) Review demographics of nonrespondents vs. respondents (size, performance, NAICS category, longevity) to test nonresponse bias, 3) Perform Split-Half reliability test, and 4) Review consistency among organization level responses relative to department and staff position. Results for each follow: 
1) The Pilot Test tests the questionnaire with no noted issues from respondents as documented in Chapter V -- Pilot Test Observations / Conclusions / Plan.

2) Review demographics of non-respondents vs. respondents (size, performance, NAICS category, longevity) to test non-response bias. Respondents are representative of the sample population as follows in Table 53: 
TABLE 53

\section{DEMOGRAPHICS OF RESPONDENTS VS. NONRESPONDENTS}

29 of 64 (45\%) NAICS Categories solicited responded

\begin{tabular}{|c|c|c|c|c|c|c|c|}
\hline $\begin{array}{l}3 \text { Digit } \\
\text { NAICS Code }\end{array}$ & $\begin{array}{l}\text { \# Contacts } 5 \\
\text { yrs wemail }\end{array}$ & $\begin{array}{l}\text { Response } \\
\mathrm{s}\end{array}$ & $\begin{array}{l}\text { Response } \\
\text { Rate }\end{array}$ & $\begin{array}{l}3 \text { DIglt } \\
\text { NAICS } \\
\text { Code }\end{array}$ & $\begin{array}{l}\text { \# Contacts } 5 \\
\text { yrs wiemail }\end{array}$ & $\begin{array}{l}\text { Response } \\
\mathrm{s}\end{array}$ & $\begin{array}{l}\text { Response } \\
\text { Rate }\end{array}$ \\
\hline 211 & 30 & 2 & $6.67 \%$ & 451 & 4 & & \\
\hline 212 & 13 & & & 452 & 6 & & \\
\hline 213 & 20 & & & 453 & 7 & & \\
\hline 221 & 49 & 5 & $10.20 \%$ & 454 & 6 & & \\
\hline 236 & 13 & & & 481 & 7 & & \\
\hline 237 & 9 & 2 & $22.22 \%$ & 484 & 10 & & \\
\hline 311 & 25 & 3 & $12.00 \%$ & 486 & 9 & & \\
\hline 312 & 10 & 1 & $10.00 \%$ & 488 & 4 & & \\
\hline 315 & 10 & & & 511 & 59 & 3 & $5.08 \%$ \\
\hline 316 & 7 & & & 512 & 5 & & \\
\hline 321 & 4 & & & 515 & 12 & 1 & $8.33 \%$ \\
\hline 322 & 12 & 2 & $16.67 \%$ & 517 & 25 & & \\
\hline 323 & 6 & & & 518 & 21 & & \\
\hline 324 & 6 & & & 522 & 221 & 14 & $6.33 \%$ \\
\hline 325 & 158 & 6 & $3.80 \%$ & 523 & 33 & & \\
\hline 326 & 11 & 1 & $9.09 \%$ & 524 & 45 & 1 & $2.22 \%$ \\
\hline 327 & 4 & & & 525 & 56 & 2 & $3.57 \%$ \\
\hline 331 & 10 & & & 531 & 9 & & \\
\hline 332 & 23 & 1 & $4.35 \%$ & 532 & 7 & & \\
\hline 333 & 54 & 4 & $7.41 \%$ & 533 & 9 & 1 & $11.11 \%$ \\
\hline 334 & 245 & 8 & $3.27 \%$ & 541 & 104 & 4 & $3.85 \%$ \\
\hline 335 & 24 & 1 & $4.17 \%$ & 551 & 12 & & \\
\hline 336 & 34 & 2 & $5.88 \%$ & 561 & 35 & 1 & $2.86 \%$ \\
\hline 337 & 9 & & & 562 & 7 & 1 & $14.29 \%$ \\
\hline 339 & 58 & 1 & $1.72 \%$ & 611 & 10 & & \\
\hline 423 & 33 & 1 & $3.03 \%$ & 621 & 21 & & \\
\hline 424 & 16 & & & 622 & 4 & & \\
\hline 441 & 7 & 1 & $14.29 \%$ & 623 & 3 & & \\
\hline 443 & 3 & 1 & $33.33 \%$ & 713 & 13 & 1 & $7.69 \%$ \\
\hline 445 & 4 & & & 721 & 5 & & \\
\hline 446 & 5 & & & 722 & 20 & 1 & $5.00 \%$ \\
\hline 448 & 16 & & & 812 & 11 & 2 & $18.18 \%$ \\
\hline
\end{tabular}

\begin{tabular}{|c|c|c|c|c|c|c|}
\hline $\begin{array}{l}\text { Performance } \\
\text { Stock Price \% Change }\end{array}$ & Count & Mean & Median & Std Dev & Max & $M$ in \\
\hline "Respondent Naics Categories & 1288 & 9.88 & -13.22 & 105.54 & 1007.75 & -99.62 \\
\hline Respondent Companies & 72 & 17.63 & -3.27 & 130.42 & 978.17 & -93.23 \\
\hline
\end{tabular}

${ }^{*}$ History not available for two companies ( $\mathrm{NA}$ for data range $\& 1 \mathrm{Ch} 11$ )

\begin{tabular}{|l|r|r|r|r|r|r|}
\hline Longevity (years) & \multicolumn{1}{|c|}{ Count } & Mean & Median & Std Dev & Max & Min \\
\hline A.ll solicited & 1731 & 14.48 & 13.5 & 6.06 & 46.92 & 5 \\
\hline Respondent Naics Categories & 1290 & 14.46 & 13.67 & 6.17 & 46.92 & 5 \\
\hline Respondent Companies & 74 & 13.81 & 13.46 & 5.09 & 27 & 5.17 \\
\hline
\end{tabular}


3) Split-Half Reliability Test - This test proposed in Chapter IV is not appropriate in this survey because the survey does not include multiple questions of the same type and quality for which to split and test for correlation. Each question is of different type and independent of the other questions, though questions 6 - 11 all relate to the construct "measurement." Therefore, each question will now be stated with steps taken to address any reliability issues.

\section{Question 1: Please enter your company code.}

Steps taken: Format is defined and is required to be submitted as 9999.999 for the survey to be accepted. Two cases are noted with incorrect company code submissions, one of which advised the error by email for correction (input Company Code 5150.099 instead of 5150.009) while the other used the sample number "9999.999" and could not be identified for inclusion in the analysis. Better reliability may have been achieved by requiring a second input of the Company Code.

Question 2: How frequently does your product change/improve? (check most appropriate)

Steps taken: Select from three alternatives or "Don't Know." There is minimal ambiguity in the operational definition of the question.

Question 3: How frequently do your technical processes change/improve? (check most appropriate) 
Steps taken: Select from three alternatives or "Don't Know." There is minimal ambiguity in the operational definition of the question.

Question 4: How frequently does your company or department change its performance $\underline{\text { measurement system? (select one) }}$

Steps taken: Select from three alternatives or "Don't Know." There may be some ambiguity in the operational definition of "performance measurement system."

Question 5: What is your position in the company? (select one)

Steps taken: Select from a set of options or "Other." There is minimal ambiguity in the operational definition of the question.

Question 6: In which department do you work? (select one)

Steps taken: Select from set of options or "Other." There is minimal ambiguity in the operational definition of the question.

Question 7: Estimate the Number of Financial Measures you use to monitor performance or which are used to measure your performance (see following sample list to help estimate number of measures used).

Steps taken: The question uses Balanced Scorecard based terminology which is familiar in industry and examples of financial measures are provided for consideration. 
Question 8: Estimate the Number of Internal Operating (Nonfinancial) Measures you use to monitor performance or which are used to measure your performance. (see following sample list to help estimate number of measures used).

Steps taken: The question uses Balanced Scorecard based terminology which is familiar in industry and examples of internal operating measures are provided for consideration.

Question 9: Estimate the Number of Employee Related (Learning and Growth; Nonfinancial) Measures you use to monitor performance; or which are used to measure your performance. (see following sample list to help estimate number of measures used).

Steps taken: The question uses Balanced Scorecard based terminology which is familiar in industry and examples of employee related measures are provided for consideration.

Question 10: Estimate the Number of Customer (Nonfinancial) Measures you use to monitor performance or which are used to measure your performance. (see following $\underline{\text { sample list to help estimate the number of measures used) }}$

Steps taken: The question uses Balanced Scorecard based terminology which is familiar in industry and examples of customer related measures are provided for consideration.

Question 11: Rank in order from most important to least important the process types related to performance measures you use or by which your performance is measured 
Process 1: Identity Processes (processes that define your company)

Process 2: Priority Processes (processes that are critical to support the identity of your company)

Process 3: Background Processes (processes that are necessary support to daily operations)

Process 4: Mandated Processes (processes necessary for regulatory compliance)

Steps taken: Process types are presented in a random ordered sequence with no possibility to select any item twice. Random sequencing of presentation and validity of response sequence is tested using 12 test company entries. Results of the test are shown in Table 54 documenting: 1) Sequence presented by instrument; 2) Ranked sequence input into survey test; and 3) Ranked sequence survey results as follows: 
TABLE 54

VERIFICATION OF RANDOM ALTERNATIVE RESPONSE SEQUENCING

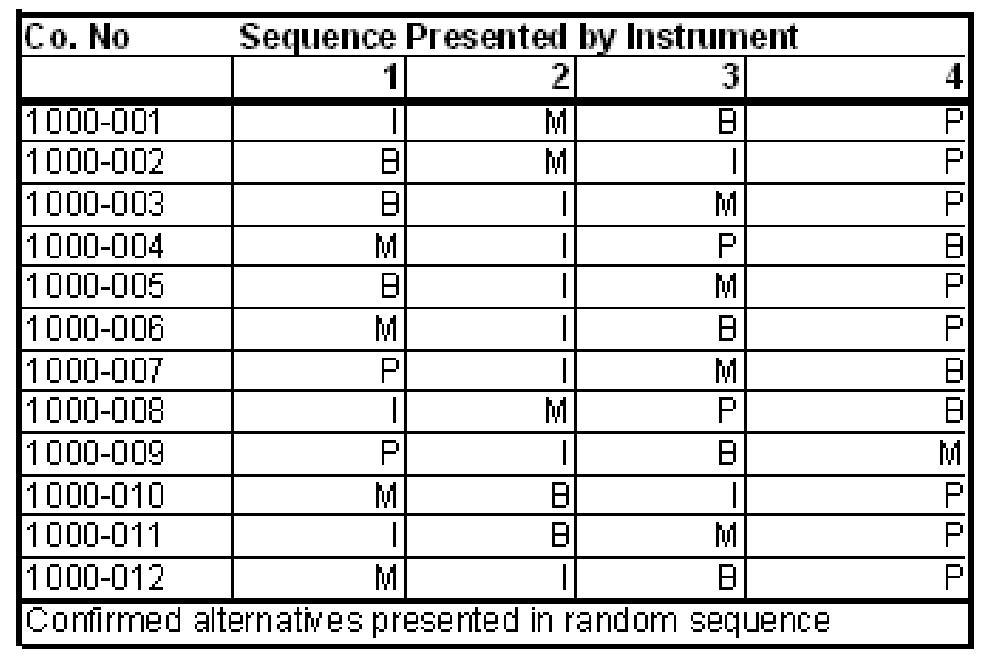

\begin{tabular}{|c|c|c|c|c|c|}
\hline Co. No. & \multicolumn{5}{|c|}{ Ranked Sequence Input into Survey Test } \\
\hline & 1 & 2 & 3 & & 4 \\
\hline $1000-001$ & & $\overline{\mathrm{P}}$ & $\overline{\mathrm{B}}$ & & $\mathrm{M}$ \\
\hline $1000-002$ & M & T & $\overline{\mathrm{P}}$ & & $\overline{\mathrm{B}}$ \\
\hline $1000-003$ & 可 & $\mathrm{M}$ & $T$ & & $\bar{P}$ \\
\hline $1000-004$ & $\mathrm{P}$ & $\mathrm{B}$ & $\mathrm{M}$ & & \\
\hline $1000-005$ & T & $\mathrm{P}$ & $\overline{\mathrm{B}}$ & & $M$ \\
\hline $1000-006$ & $M$ & 1 & $\mathrm{P}$ & & $\mathrm{E}$ \\
\hline $1000-007$ & 目 & $\mathrm{M}$ & $\mathrm{T}$ & & $\bar{P}$ \\
\hline $1000-008$ & $\mathrm{P}$ & $\mathrm{E}$ & $M$ & & 1 \\
\hline $1000-009$ & 4 & $\mathrm{P}$ & $\mathrm{B}$ & & $\bar{M}$ \\
\hline $1000-010$ & iin & 1 & $\mathrm{P}$ & & $\overline{\mathrm{B}}$ \\
\hline $1000-011$ & 可 & $M$ & $T$ & & $\bar{P}$ \\
\hline $1000-012$ & $\mathrm{P}$ & $\mathrm{B}$ & M & & \\
\hline
\end{tabular}

\begin{tabular}{|c|c|c|c|c|c|}
\hline Co. No. & RankedSe & equence St & Survey Resu & & \\
\hline & 1 & & 2 & $\overline{4}$ & $\overline{4}$ \\
\hline $1000-001$ & & & PI & $M$ & \\
\hline $1000-002$ & $\mathrm{~m}$ & & $\mathrm{P}$ & $\overline{\mathrm{B}}$ & \\
\hline $1000-003$ & 可 & $M$ & 1 & $\bar{P}$ & \\
\hline $1000-004$ & $\mathrm{P}$ & $\mathrm{B}$ & $M$ & & \\
\hline $1000-005$ & 1 & & $\mathrm{~B}$ & $M$ & \\
\hline $1000-006$ & m & 1 & $\mathrm{P}$ & $\mathrm{E}$ & \\
\hline $1000-007$ & 目 & M & 1 & $\bar{P}$ & \\
\hline $1000-008$ & $\mathrm{P}$ & $\mathrm{B}$ & $M$ & & \\
\hline $1000-009$ & 1 & $\mathrm{P}$ & $\mathrm{B}$ & $M$ & \\
\hline $1000-010$ & Mi & & $\mathrm{P}$ & $\mathrm{B}$ & \\
\hline $1000-011$ & 耐 & $M$ & 1 & $\bar{P}$ & \\
\hline $1000-012$ & $\mathrm{P}$ & E & $M$ & ni & \\
\hline Confilmen & s consiste & tent with res & spondent rar & ankin & \\
\hline
\end{tabular}


Unlike the other questions, the operational definition of Question 11 contains ambiguity and is subject to interpretation. The ambiguity in the question, or way to respond, is confirmed by the comments from two respondents as follows: Respondent 56 "Didn't really understand Question 11 definitions;" and Respondent 73 "Seems difficult to rank the processes individually as hopefully the company's identity is based on the premise of providing quality service daily; which is designed to meet regulatory requirements. Therefore; they all work together and by focusing on one of the processes; we are also addressing the other processes at the same time."

Question 11 is placed last in the survey because of anticipated difficulty. The survey format did not allow full definition or explanation of the processes under consideration, and the difficulty and potential difference of interpretation are noted by Keen (1997) as follows:

Analyzing the salience of a firm's processes is an important task that requires considerable thought and insight.... As we have seen, one of several complexities to be considered is that different groups and individuals see the salience of the same process differently. So the question "Whose valuation counts most? must be answered before a process's importance to the entire firm can be determined (p. 54).

\section{Strengths}

Shepherd and Günter (2006) state that there is a need for performance measurement comparisons across market sectors since most studies are conducted within 
a specific market sector. This study is representative of a broad range of industries, with respondents representing 29 different 3-digit NAICS categories as noted in Table 55:

TABLE 55

\section{RESPONDING COMPANY NAICS CATEGORY DESCRIPTIONS}

\begin{tabular}{|cl|}
\hline 3 Digit & \\
NAICS & \multicolumn{1}{c|}{ Responding Company NAICS Category Descriptions } \\
Code & \\
\hline 211 & Oil and Gas Extraction \\
221 & Utilities \\
237 & Heavy and Civil Engineering Construction \\
311 & Food Manufacturing \\
312 & Beverage \& Tobacco Product Manufacturing \\
322 & Paper Manufacturing \\
325 & Chemical Manufacturing \\
326 & Plastics and Rubber Products \\
332 & Fabricated Metal Product \\
333 & Machinery Manufacturing \\
334 & Computer and Electronic Product \\
335 & Electrical Equipment, Appliance, and Component \\
336 & Transportation Equipment Manufacturing \\
339 & Miscellaneous Manufacturing \\
423 & Merchant Wholesalers, Durable Goods \\
441 & Motor Vehicle and Parts Dealers \\
443 & Electronics and Appliance Stores \\
511 & Publishing Industries (except Internet) \\
515 & Broadcasting (except Internet ) \\
522 & Credit Intermediation and Related Activities \\
524 & Insurance Carriers and Related Activities \\
525 & Funds, Trusts, and Other Financial Vehicles \\
533 & Lessors of Nonfinancial Intangible Assets (except Copyrighted Works) \\
541 & Professional, Scientific, and Technical Services \\
561 & Administrative and Support Services \\
562 & Waste Management \&Remediation Services \\
713 & Amusement, Gambling, \& Recreation Industries \\
722 & Food Services and Drinking Places \\
812 & Personal and Laundry Services \\
\hline & \\
\hline
\end{tabular}


Hypotheses 2, 3, and 4 results are based on respondents indicating the number of measures they use in each of 4 (Balanced Scorecard) categories with sample metrics provided for consideration as follows:

1) Financial Measures: Sales; Capital Expenditures; Maintenance Expenditures; Operating Expenses; SG\&A Expenses; Product Quality Costs (warranty costs); ROI; ROA; Total Manufacturing Costs; Labor Costs; Material Costs; Indirect (overhead) Costs; Manufacturing Process Improvement Costs...

2) Internal Operating (Non-Financial) Measures: Information Technology (\% cost); New Product \% of Sales; Proprietary Products \% of Sales; New Product Introduction vs Competitors; Manufacturing Process Capabilities; Time to Develop Next Generation of Products; Product quality (defect rates); Delivery (on time); Manufacturing Efficiency; Suppliers (quality, defect rates, dependability, on time delivery); Suppliers (number of); R\&D (new product introduction cycle time); Production Volume; Labor Productivity (hours used, available, overtime); Machine Productivity (hours running, available, downtime); Material Usage (inefficiency, waste); Setup Efficiency (setup time, number of setups); Manufacturing Cycle Time (total process time); Inventory (turnover); Product Defects (number of errors, rework, scrap)...

3) Learning and Growth (Employee related Non-Financial) Measures: Safety (number of accidents, injuries); Employee Satisfaction (surveys, grievances); Employee Skills (level of education, experience); Employee Empowerment (\# suggestions, \# of improvement teams); Employee Training / Education (hours or time allocated for training); Employee Loyalty / Turnover; Absenteeism; Employee Perception of Leadership...

4) Customer (Non-Financial) Measures: Customer Acquisition (\# new, \% sales from new); Customer Retention / Loyalty (\# repeat customers); Customer Satisfaction (surveys, complaints); Phone System Utility (automated, response time); Market Share; Time to Fill Customer Orders; Deliver Performance (ontime, \% correct delivery); Time to Respond to Customer Problems; Flexibility / Responsiveness (ability to vary product)...

Deriving the number of financial vs. nonfinancial measures used, and the number of categories of balanced scorecard metrics used from these defined measures increases 
reliability by providing clarity of the operational definition for each of the questions, thereby minimizing the potential of questionable understanding or submission of a casual estimate of an aggregate / general response.

In summary, strengths of this study include:

- A wide range of industries represented in responses to the survey

- Performance (dependent variable) based on stock price change over a five-year period

- Adjusted for dividends and splits

- Based on one-year rolling average to eliminate year-end and seasonal distortions

- Standardized based on 3-digit NAICS Code to eliminate industry variance

- Respondent demographics are comparable to the sample population

- Clarity in the operational definition of questions regarding financial, nonfinancial and balanced scorecard measurement systems

\section{Suggestions for Further Research}

In conclusion, responses from companies in broad range of industries are received and compared for performance based on change in stock price over a five-year time frame using a one-year rolling average to eliminate seasonal or year-end distortions adjusted for dividends and splits. Results of the research support the hypothesis that nonfinancial measures correlate more positively to firm value than financial measures, and that the number of categories of balanced scorecard metrics used correlate to firm value. These results, however, do not address specific measurements, statistical validity of the measurements, or linkages between selected measurements and strategy. There also is no assurance that the "number of measures" in any category defined by respondents in this study reflects the weight placed on the value of those measures for 
decision making. Further study regarding specific measurements utilized, weighting of the measurements applied by decision makers, statistical validity of the measurements, and linkages between measurements and strategy may provide further insights into characteristics of effective performance measurement systems.

Statistical significance is not achieved in $\mathrm{H}_{1}$ and this is the area which offers the greatest potential for exploration. The following changes to methodology are recommended: 1) Utilize a targeted solicitation campaign with specifically defined company contact emails (rather than the general company emails utilized in this research) to increase the response rate; 2) Provide well articulated definition and explanation of the processes under consideration to reduce ambiguity; and 3) Request ranking of the processes using multiple questions stated in different ways to increase measurable reliability using the split half reliability test.

Finally, as noted by Shepherd and Günter (2006) “...it is important to treat measurement systems as dynamic entities that must respond to environmental and strategic change. Consequently, further work is needed to investigate the factors influencing the evolution of performance measurement systems ... and how to handle their ongoing maintenance" (p. 253). 


\section{BIBLIOGRAPHY}

Abernethy, M. A., \& Brownell, P. (1997). Management Control Systems in Research and Development Organizations: The Role of Accounting, Behavior and Personnel Controls, Accounting, Organizations and Society, 22(3/4), 233-248.

Abernethy, M. A., Lillis, A. M. (1995). The Impact of Manufacturing Flexibility on Management Control System Design. Accounting, Organizations and Society, 20(4), 241-258.

Allen, F., Bernardo, A., \& Welch, I. (2000). A Theory of Dividends Based on Tax Clienteles. Journal of Finance, 55(6), 2444-2536.

Altman, E.. (1984). A Further Empirical Investigation of the Bankruptcy Cost Question. Journal of Finance, 1067-1089.

Amir, E., \& Lev, B. (1996). Value-relevance of Nonfinancial Information: The Wireless Communications Industry. Journal of Accounting and Economics, 22, 3-30.

Anderson, S. W., \& Young, S. M. (1999). The Impact of Contextual and Process Factors on the Evaluation of Activity-Based Costing Systems, Accounting, Organizations and Society, 24, 525-559.

Ang, J., \& Peterson. (1984). The Leasing Puzzle. Journal of Finance. 1055-1065.

Atkinson, A. A., \& Shafir, W. (1998). Standards for Field Research in Management Accounting, Journal of Management Accounting Research, 10, 41-68.

Baker, G., Gibbons, R., \& Murphy, K. J. (1994). Subjective Performance Measures in Optimal Incentive Contracts. The Quarterly Journal of Economics, 1125-1156.

Baker, M., \& Wurgler, J. (2002). Market Timing and Capital Structure. Journal of Finance, 57, 1-32.

Banker, R. D., Potter, G., \& Srinivasan, D. (2000). An Empirical Investigation of an Incentive Plan that Includes Nonfinancial Performance Measures. The Accounting Review, 75(1), 65-92.

Bell, L., \& Jenkinson, T. (2002). New Evidence of the Impact of Dividend Taxation and on the Identity of the Marginal Investor, Journal of Finance.57 (3), 13211346.

Biddle, G. C., Bowen, R. M., \& Wallace, J. S. (1997). Does EVA ${ }^{\circledR}$ Beat Earnings? Evidence on Associations with Stock Returns and Firm Values, Journal of Accounting \& Economics, 24, 301-336.

Billet, M. T., \& Mauer, D. C. (2000). Diversification and the Value of Internal Capital Markets: The Case of Tracking Stock, Journal of Banking and Finance,24, 1457-1490.

Black, F., \& Scholes, M. (1972). The Valuation of Option Contracts and a Test of Market Efficiency, Journal of Finance, 399-418.

Blalock, H. M. Jr. (1972). Social Statistics. NY: McGraw-Hill, Inc.

Bloom, M., \& Milkovich, G. T. (1998). Relationships Among Risk, Incentive Pay, and Organizational Performance, The Academy of Management Journal, 41(3), 283-297.

Bourne, M., Kennerley, M., \& Franco-Santos, M., (2005). Managing Through 
Measures: A Study of Impact on Performance. Journal of Manufacturing

Technology Management, 16(4), 373-395.

Bouwens, J., \& Abernethy, M. A. (2000). The Consequences of Customization on

Management Accounting System Design, Accounting, Organizations and Society, 25, 221-241.

Boyd, G. A. (2006). Development of a Performance-Based Industrial Energy

Efficiency Indicator For Cement Manufacturing Plants. Decision and

Information Sciences Division, Argonne National Laboratory, Sponsored by

U.S. Department of Energy, Managed by Chicago University, ANL/DIS-06-3, 1-24.

Bromwich, M. (1990). The Case for Strategic Management Accounting: The role of Accounting Information for Strategy in Competitive Markets, Accounting, Organizations and Society, 15(1/2), 27-46.

Brown, M. G., Hitchcock, D. E., \& Willard, M. L. (1994). Why TQM Fails and What to do About It. New York: Irwin Professional Publishing.

Bushman, R. M., Indjejikian, R. J., \& Smith, A. (1995). Aggregate Performance Measures in Business Unit Manager Compensation: The Role of Intrafirm Interdependencies, Journal of Accounting Research, 33, 101-128.

Capon, N., Farley, J. U., \& Hoenig, S. (1990). Determinants of Financial Performance: A Meta-Analysis. Management Science, 36(10), 1143-1159.

Carrillo, J. E. (2005). Industry Clockspeed and the Pace of New Product Development, Production and Operations Management, 14 (2), 125-141.

Chapman, C. S. (1997). Reflections on a Contingent View of Accounting, Accounting, Organizations and Society, 22(2), 189-205.

Chenhall, R. H. (1997). Reliance on Manufacturing Performance Measures, Total Quality Management and Organizational Performance, Management Accounting Research, 8, 187-206.

Chenhall, R. H. (2003). Management Control Systems Design Within Its Organizational Context: Findings From Contingency-Based Research and Directions for the Future, Accounting, Organizations and Society, 28, 127-168.

Chenhall, R. H., \& Langfield-Smith, K. (1998). The Relationship Between Strategic Priorities, Management Techniques and Management Accounting: An Empirical Investigation Using a Systems Approach, Accounting, Organizations and Society, 23 (3), 243-264.

Chirinko, A., \& Singha, A. (2000). Testing Static Tradeoff Against Pecking Order Models of Capital Structure: A Critical Comment, Journal of Financial Economics, 58(3), 412-426.

Chow, C. W., Shields, M. D., \& Chan, Y. K. (1991). The Effects of Management Controls and National culture on Manufacturing Performance: An Experimental Investigation, Accounting, Organizations and Society,16(3), 209226.

Chow, C. W., \& Van der Stede, W. A. (2006). The Use and Usefulness of Nonfinancial Performance Measures. Management Accounting Quarterly, 7(3), $1-8$.

Collins, J. C., \& Porras, J. I. (1997). Built to Last: Successful Habits of Visionary 
Companies. New York: Harper Business.

Cooper, R., \& Kaplan, R. S. (1988). Measure Costs Right: Make the Right Decision. Harvard Business Review 66(5), 96-103.

Cooper, R., \& Kaplan, R. S. (1991). Profit Priorities from Activity-Based Costing. Harvard Business Review, 69(3), 130-135.

Copeland, T. E., Weston, J. F., \& Shastri, K. (2005). Financial Theory and Corporate Policy (4th ed.). New York: Pearson Addison Wesley.

Damodaran, W. (2002). Investment Valuation: Tools and Techniques for Determining the Value of Any Asset(2nd ed.). New York: John Wiley \& Sons, Inc.

Datar, S., Kulp, S. C., \& Lambert, R. A. (2001). Balancing Performance Measures, Journal of Accounting Research, 39 (1), 75-92.

Davidson, S., \& Weil, R. L. (1978). Handbook of Cost Accounting. New York: McGraw-Hill, Inc.

Davila, T. (2000). An Empirical Study on the Drivers of Management Control Systems' Design in New Product Development, Accounting, Organizations and Society, 25, 383-409.

Davis, J. H., Schoorman, D., \& Donaldson, L. (1997). Toward a Stewardship Theory of Management, The Academy of Management Review, 22(1), 20-47.

DeAngelo, H., DeAngelo, L., \& Skinner, D. (2000). Special Dividends and the Evolution of Dividend Signaling, Journal of Financial Economics, 57(3), 309354.

DePaolo, C. A., \& Sherwood, A. L. (2006). Instructional Uses of Web-Based Survey Software. The Journal of Educators Online, 3(1), 1-19. Retrieved May 22, 2007 from http://www.thejeo.com/Archives/Volume3Number1/DePaoloFinal.pdf.

Dickinson, J. R. (2007). The Invalidity of Profit=F(Product Quality) PIMS Validation of Marketing Games. Developments in Business Simulation and Experiential Learning, 34, 287-293.

Diehl, E., \& Sterman, J. D. (1995). Effects of Feedback Complexity on Dynamic Decision Making. Organizational Behavior and Human Decision Processes, 62(2), 198-215.

Dillman, D. (2000). Mail and Internet Surveys: The Tailored Design Method (2nd ed.). New York: John Wiley \& Sons, Inc.

Drazin, R., \& Van de Ven, A. H. (1985). Alternative Forms of Fit in Contingency Theory, Administrative Science Quarterly, 30(4), 514-539.

El-Mashaleh, M. S., Minchin Jr., R. E., \& O'Brien, W. J. (2007). Management of Construction Firm Performance Using Benchmarking. Journal of Management in Engineering, 23(1), 10-17.

Fama, E. F., \& French, K. (1992). The Cross-Section of Expected Stock Returns, Journal of Finance, 47, 427-466.

Fama, E. F., \& French, K. (2002). Testing Trade-Off and Pecking Order Predictions about Dividends and Debt, Review of Financial Studies, 15, 1-33.

Feltham, G. A., Xie, J. (1994). Performance Measure Congruity and Diversity in Multi-Task Principal/Agent Relations, The Accounting Review, 69 (3), 429453. 
Fine, C. H. (1998). Clockspeed: Winning Industry Control in the Age of Temporary Advantage. New York: Perseus Books.

Fischer, S. (1978). Call Option Pricing When the Exercise Price is Uncertain and the Valuation of Index Bonds, Journal of Finance, 33(1), 169-186.

Fleischman, R. K., \& Tyson, T. N. (1997). Developing Expertise: Two Episodes in Early Nineteenth Century U.S. Management Accounting History. Business and Economic History, 26(2), 365-380.

Forrester, J. W. (1958). Industrial Dynamics: A Major Breakthrough for Decision Makers, Harvard Business Review, 37-66.

Frazelle, E. (2002). Supply Chain Strategy, The Logistics of Supply Chain Management. New York: McGraw-Hill.

Goosen, K. R., Wolfe, J, \& Gold, S. (2007). Panel Discussion: Alternative Ways of Using the Internet for Business Simulations to Input Decisions, Process, and Present Financial and Economic Data Output. Developments in Business Simulation and Experiential Learning, 34, 67-68.

Grullon, G., \& Michaely, R. (2002). Dividends, Share Repurchase and the Substitution Hypothesis, The Journal of Finance, 57(4), 1649-1684.

Green, P. E., Tull, D. S., Albaum, G., (1988). Research For Marketing Decisions (5th ed.). Prentice-Hall.

Guimaraes, T., Cook, D., \& Natarajan, N. (2002). Exploring the Importance of Business Clockspeed as a Moderator for Determinants of Supplier Network Performance, Decision Sciences, 33 (4), 629-644. Retrieved January 14, 2008 from http://www. allbusiness.com/accounting-reporting/cost-accountingdecision-theory/1028683-1.html

Gunn, H. (2002). Web-based Surveys: Changing the Survey Process. First Monday, 7 (12), 1-14. [Peer-Reviewed Journal on the Internet]. Retrieved May 25, 2007 from http://www.firstmonday.org/issues/issue 7_12/gunn/.

Hall III, A. D. (1989). Metasystems Methodology: A New Synthesis and Unification. New York: Pergamon Press.

Haponova, T., Al-Jobouri, S., \& Reymen, I. (2006). Process Performance Indicators in Project Pre-Design Stage. University of Twente, Eidnhoven The Netherlands. [Electronic Edition] Retrieved May 6, 2007 from http://www.ctw.utwente.nl/staff/CME/I.M.M.J.Reymen/publication/06_Hapona va.PDF.

Heier, J. R. (2000). The Foundations of Modern Cost Management: The Life and Work of Albert Fink. Accounting Business \& Financial History, (10)2, 213-243.

Retrieved May 24, 2007 from http://econpapers.repec.org/article/tafacbsfi/v_3A10_3Av_3A2000_3Ai_3A2_ 3Ap_3A213-243.htm.

Hemmer, T. (1996). On the Design and Choice of "Modern" Management Accounting Measures, Journal of Accounting Management Research, 8, 87-116.

Heskett, J. L., Jones, T. O., Loveman, G. W., Sasser, Jr. W. E., \& Schlesinger, L. A. (1994). Putting the Service-Profit Chain to Work, Harvard Business Review, March-April, 164-170.

Howell, D. C. (2002). Statistical Methods for Psychology (5th ed.). Belmont: 
Thomson Wadsworth.

Hull, R. (2007). Financial Management, Chapter 18, Managing Dividend Policy.

Washburn University. Topeka. Retrieved 9/21/07 from http://www.washburn.edu/sobu/rhull/cf18.html.

Ittner, C. D., \& Larcker, D. F. (1998). Innovations in Performance Measurement: Trends and Research Implications, Journal of Management Accounting Research, 10, 205-238.

Ittner, C. D., \& Larcker, D. F. (2003). Coming Up Short on Nonfinancial Performance Measurement, Harvard Business Review, Reprint R0311F, 1-8.

Ittner, C. D., Larcker, D. F., \& Meyer, M. W. (2003). Subjectivity and the Weighting of Performance Measures: Evidence From a Balanced Scorecard, The Accounting Review, 78 (3), 725-758.

Johnson, H. T. (1981). Toward a New Understanding of Nineteenth-Century Cost Accounting. The Accounting Review, LVI(3), 510-518.

Johnson, H. T., \& Broms, A. (2000). Profit Beyond Measure. New York: The Free Press.

Johnson, H. T., \& Kaplan, R. S. (1987). Relevance Lost, The Rise and Fall of Management Accounting. Boston: Harvard Business School Press.

Kaplan, R. S. (1983). Measuring Manufacturing Performance: A New Challenge for Managerial Accounting Research. The Accounting Review, 58 (4), 686-705.

Kaplan, R. S. (1984a). The Evolution of Management Accounting. The Accounting Review, 59(3), 390418.

Kaplan, R. S. (1984b). Yesterday's Accounting Undermines Production. Harvard Business Review, 62(4), 95-101.

Kaplan, R. S., \& Norton, D. P. (1996). The Balanced Scorecard. Boston: Harvard Business School Press.

Kaufold, H., \& Smirlock, M. (1986). Managing Corporate Exchange and Interest Rate Exposure, Financial Management, 15(3), 64-72.

Keen, P. G. W. (1997). The Process Edge: Creating Value Where It Counts. Boston: Harvard Business School Press.

Kemsley, D., \& Nissim, D. (2002). Valuation and the Debt Tax Shield, Journal of Finance, 57(5), 2045-2073.

Lee, H., Kwak, W., \& Han, I. (1995). Developing a Business Performance Evaluation System: An Analytic Hierarchical Model. The Engineering Economist, 40(4), 343-357.

Lee, Y.T. (2001). Management Data Specification for Supply Chain Integration. U.S. Department of Commerce Technology Administration, National Institute of Standards and Technology. Gaithersburg

Leenders, M. R., Johnson, P. F., Flynn, A. E., \& Fearon, H. E. (2006). Purchasing and Supply Management (13th ed.). New York: McGraw-Hill Irwin.

Lendaris, G. G. (1986). On (No Suggestions) and the Problem Solver: Tutorial Comments. IEEE Transactions on Systems, Man, and Cybernetics, 16(4), 604610.

Lillis, A. M. (2002). Managing Multiple Dimensions of Manufacturing Performance -An Exploratory Study, Accounting, Organizations and Society, 27, 497-529. 
Linestone, H. A. (1999). Decision Making for Technology Executives: Using Multiple Perspectives to Improve Performance. Boston: Artech House.

Locke, D. (1996). Global Supply Management. New York: McGraw-Hill.

Mendelson, H., \& Pillai, R. R. (1999). Industry Clockspeed: Measurement and Operational Implications, Manufacturing \& Service Operations Management, 1 (1), 1-20.

Mitroff, I. I., \& Turoff, M. (1973). Technological Forecasting and Assessment: Science and/or Mythology, Technological Forecasting and Social Change, 5, 113-134.

Modigliani, F., \& Miller, M. H. (1958). The Cost of Capital, Corporation Finance, and the Theory of Investment, American Economic Review, 261-297.

Moers, F. (2005). Discretion and Bias in Performance Evaluation: The Impact of Diversity and Subjectivity, Accounting, Organizations and Society, 30, 67-80.

Moor, Aldo de, \& Smits, M. (2002). Key Performance Indicators for Knowledge Management in a Community of Practice. Tilburg University, Metis.

Retrieved May 6, 2007 from http://www.starlab.vub.ac.be/staff/ademoor/papers/ti02.pdf, 1-29.

Moores, K., \& Yuen, S. (2001). Management Accounting Systems and Organizational Configuration: A Life-Cycle Perspective, Accounting, Organizations and Society, 26,251-389.

Nagar, V., \& Rajan, M. V. (2001). The Revenue Implications of Financial and Operational Measures of Product Quality. The Accounting Review, 76(4), 495513.

Neely, A., Kennerley, M., \& Martinez (2004). Does the Balanced Scorecard Work: An Empirical Investigation, Cranfield University, Retrieved September 8, 2010 from http://www.som.cranfield.ac.uk/som/dinamiccontent/research/cbp/NEELY\%20KENNERLEY $\% 20$ MARTINEZ\%20Does\%20the\%20Balanced\%20Scorecard $\% 20$ Work\%20EurOMA.pdf

Neely, A., Mills, J., Platts, K., Richards, H., Gregory, M., Bourne, M., \& Kennerley, M. (2000). Performance Measurement System Design: Developing and Testing a Process-Based Approach, International Journal of Operations \& Production Management, 20(10), 1119-1145.

Neely, A., Richards, H., Mills, J., Platts, K., \& Bourne, M. (1997). Designing Performance Measures: A Structured Approach, International Journal of Operations \& Production Management, 17(11), 1131-1152.

Osama, Athar (2006). Multi-Attribute Strategy and Performance Architectures in R\&D (Doctoral dissertation, Pardee Rand Graduate School, 2006). Rand Corporation, Santa Monica.

Otley, D. (1999). Performance Management: A Framework for Management Control Systems Research, Management Accounting Research, 10, 363-382.

Pagels, H. R. (1988). Dreams of Reason: The Computer and the Rise of the Sciences of Complexity. New York: Bantam Books.

Palia, A. (2007). Online Budgeting and Marketing Control with the Proforma Analysis Package. Developments in Business Simulation and Experiential Learning, 34, 
149-154.

Pearce, J. L., Stevenson, W. B., \& Perry, J. L. (1985). Managerial Compensation Based on Organizational Performance: A Time Series Analysis of the Effects of Merit Pay, The Academy of Management Journal, 28(2), 261-278.

Perera, S., Harrison, G., \& Poole, M. (1997). Customer-Focused Manufacturing Strategy and the Use of Operations-Based Nonfinancial Performance Measures: A Research Note, Accounting, Organizations and Society, 22 (6), 557-572.

Ramachandran, N. (2004). Informativeness of Performance Measures in the Presence of Reporting Discretion, Journal of Accounting, Auditing \& Finance, 19(1), 6183.

Roztocki, N. (2000a). The Integrated Activity-Based Costing and Economic Value Added Information System. Proceedings of the Society for Advancement of Management (SAM) 2000 International Management Conference, St.

Augustine, Florida., March 30 - April 1, 2000.

Roztocki, N. (2000b). Implementing an Integrated Activity-Based Costing and Economic Value Added System: A Case Study. Proceedings from the Industrial Engineering Research '2000 Conference, Cleveland, Ohio, May 2224, 2000. Retrieved August 16, 2006 from http:/www2.newpalz.edu/ roztockn/cleveland00.htm.

Roztocki, N. (2000c). The Integrated Activity-Based Costing and Economic Value Added System as a Strategic Management Tool: A Field Study. 2000 Pacific Conference on Manufacturing Proceedings. Southfield-Detroit, MI, USA, September 6-8, 2000, 84 - 89.

Roztocki, N. (2001a). The Integrated Activity-Based Costing and Economic Value Added System for the Service Sector. Proceedings of the International Conference on Service Management, March 22 - March 23, 2001, Angers, France, 387-397.

Roztocki, N. (2001b). Activity-Based Costing for E-Commerce. Proceedings from the Industrial Engineering Research 2001 Conference, May 20-23, 2001, Dallas, TX.

Roztocki, N. (2001c). Activity-Based Costing for E-Business. Proceedings, 2, Papers Presented at PICMET '01, Portland, Oregon - USA, July 29 - August 2, 2001.

Roztocki, N. (2001d). Using the Integrated Activity-Based Costing and Economic Value Added Information System for Project Management. Proceedings of the Seventh American Conference on Information Systems. August 2-3, 2001, Boston, MA, USA, 1454-1460.

Roztocki, N. (2003). Activity-Based Management for E-Commerce. WeB 2003 Proceedings. Seattle, WA, December 13-14, 2003, 121-129.

Roztocki, N., \& Needy, K. L. (1998). An Integrated Activity-Based Costing and Economic Value Added System as and Engineering Management Tool for Manufacturers. 1998 ASEM National Conference Proceedings. Virginia Beach, October 1-3, 1998, 77-84.

Roztocki, N., \& Needy, K. L. (1999a). Integrating Activity-Based Costing and Economic Value Added in Manufacturing. Engineering Management Journal, 


\section{$11(2), 17-22$.}

Roztocki, N., \& Needy, K. L. (1999b). EVA For Small Manufacturing Companies. Society for Advancement of Management (SAM) 1999 International Management Conference. Las Vegas, Nevada, March 28-30, 1999.

Roztocki, N., \& Needy, K. I. (1999c). How to Design and Implement an Integrated Activity-Based Costing and Economic Value Added System. Proceedings from the Industrial Engineering Research '99 conference. Phoenix, AZ, May 23-25, 1999.

Roztocki, N., \& Needy, K. L. (2000). Variation in Production Volume: The Impact of Using and Integrated ABC-and-EVA System to Reduce Distortions in Product Costs. Proceedings of the 2000 American Society for Engineering Management (ASEM) National Conference, Washington DC, October 4 - October 7, 2000, 341-346.

Roztocki, N. \& Schultz, S. M. (2003). Adoption and Implementation of Activity-Based Costing: A Web-Based Survey. Proceedings of the 12th Annual Industrial Engineering Research Conference (IERC 2003). Portland, OR, May 18-20, 2003.

Roztocki, N., Valenzuela, J. F., Porter, J. D., Monk, R. M. \& Needy, K. L. (1999). A Procedure for Smooth Implementation of Activity Based costing in Small Companies. 1999 ASEM National conference Proceedings. Virginia Beach, October 21-23, 1999, 279-288.

Roztocki, N., \& Weistroffer, H. R. (2004a). Evaluating Information Technology Investments in Emerging Economies Using Activity-Based Costing. Electronic Journal of Information Systems in Developing Countries (EJISDC), 19 (2), 1-6.

Roztocki, N. \& Weistroffer, H. R. (2004b). Using Activity-Based Costing for Evaluating Information Technology Related Investments in Emerging Economies: A Framework. Proceedings of the Tenth Americas Conference on Information Systems (AMCIS 2004). Tampa, FL, August 8-10, 2004, 642-645.

Roztocki, N., \& Weistroffer, H. R. (2005). Evaluating Information Technology Investments: A Fuzzy Activity-Based Costing Approach. Journal of Information Science and Technology (JIST), 2(4), 30-43.

Roztocki, N., \& Weistroffer, H. R. (2006). Stock Price Reaction to Investments in Information Technology: The Relevance of Cost Management Systems. The Electronic Journal Information Systems Evaluation, 9 (1), 27-30, available online at www.ejise.com. Retrieved August 16, 2006.

Ruback, R. S. (2002). Capital Cash Flows: A Simple Approach to Valuing Risky Cash Flows, Financial Management, 31, 85-103.

Rubinstein, M. F. (1975). Patterns of Problem Solving. New Jersey: Prentice-Hall, Inc.

Said, A. A., HassabElnaby, H. R., \& Wier, B. (2003). An Empirical Investigation of the Performance Consequences of Nonfinancial Measures, Journal of Management Accounting Research, 15, 193-223.

Schmenner, R. W. (1990). Production/Operations Management: Concepts and Situations(4th ed.). New York: MacMillan Publishing Company.

Schmidt, W. C. (1997). World-Wide Web Survey Research: Benefits, Potential Problems, and Solutions. Behavior Research Methods, Instruments \& 
Computers, 29(2), 274-279.

Senge, P. M. (1990). The Fifth Discipline: The Art \& Practice of The Learning Organization. New York: Doubleday.

Shank, J. K. \& Govindarajan, V. (1993). Strategic Cost Management, The New Tool for Competitive Advantage. New York: The Free Press.

Shepherd, C., \& Günter, H. (2006). Measuring Supply Chain Performance: Current Research and Future Directions. International Journal of Productivity and Performance Management, 55(3/4), 242-258.

Simon, H. (1981). The Sciences of the Artificial (2nd ed.). Cambridge: The MIT Press.

Simons, R. (1991). Strategic Orientation and Top Management Attention to Control Systems, Strategic Management Journal, 12(1), 49-62.

Sterman, J. D. (1989). Modeling Managerial Behavior: Misperceptions of Feedback in a Dynamic Decision Making Experiment, Management Science, 35(3), 321339.

Sterman, J. D. (2000). Business Dynamics: Systems Thinking and Modeling for a Complex World. New York: McGraw Hill.

Sterman, J.D., Repenning, N. P., Kofman, F. (1997). Unanticipated Side Effects of Successful Quality Programs: Exploring a Paradox of Organizational Improvement, Management Science, 43(4), 503-521.

Stewart III, G. B. (1991). The Quest for Value: The EVA ${ }^{T M}$ Management Guide. New York: Harper Business.

Stickney, C. P., Brown, P. R., \& Wahlen, J. M. (2004). Financial Reporting and Statement Analysis: A Strategic Perspective (5th ed.). Mason: South-Western.

Taggert, R. A. Jr. (1977). A Model of Corporate Financing Decisions, Journal of Finance, 1467-1500.

Thavikulwat, P. (2007). Demonstration of a Computer-Assisted Global Business Simulation. Developments in Business Simulation and Experiential Learning, 34, 111-112.

Thavikulwat, P., \& Chang, J. (2007). Applying .Net Remoting to a Business Simulation. Developments in Business Simulation and Experiential Learning, 34, 113-118.

Thysen, J., Israelsen, P. \& Jorgensen, B. (2006). Activity-Based Costing as a Method for Assessing the Economics of Modularization--A Case Study and Beyond. International Journal of Production Economics, 103, 252-270.

Van der Stede, W. A., Chow, C. W., \& Lin, T. W. (2006). Strategy, Choice of Performance Measures, and Performance. Behavioral Research in Accounting, 18, 185-205.

Zheng, H., Zhang, G., \& Park, S. H. (1995). Control Charts for Low-Volume Manufacturing. Total Quality Management, 6 (1), 91-99. 


\section{APPENDICES}

\section{Appendix A: The Firm-A Systems Perspective (Figure 1) Detailed Description with Citations}

Starting at the bottom of Figure 1, the firm is a subsystem of a larger value chain system. The value chain, comprised of upstream (Pre-Firm Value Chain) and downstream (Post-Firm Value Chain) subsystems, provides value to the metasystem environment. The firm attempts to optimize its performance based on relationships and information flow with other firms (subsystems) in the value chain (Lee, Y.T., 2001; Leenders, Johnson, Flynn \& Fearon, 2006; Locke, 1996) using a number of financial and nonfinancial performance measures (Frazelle, 2002; Shank \& Govindarajan, 1993).

Assets are managed by the firm using a variety of processes to generate cash flow. For the purpose of this research, business processes considered are Identity, Priority, Background and Mandated processes which are "...coordinated activities that involve people, procedures, and technology" (Keen, 1997, p. 13). These processes are used throughout the firm system. All processes require the use of resources (cash flow), but not all processes make a positive contribution to cash flow. The firms' cash flow from Operating activities is impacted by the use of associated controls and measures (Amir \& Lev, 1996; Baker, Gibbons \& Murphy, 1994; Banker, Potter,\& Srinivasan, 2000; Bourne, Kennerley, \& Franko-Santos, 2005; Boyd, 2006; Schmenner, 1990; Zheng, H., Zhang, G., \& Park, S. H.,1995 ). Many of these controls are designed to improve product/service 
quality, but it should be noted that profit is not necessarily a function of quality (Dickinson, 2007; Sterman, Repenning, \& Kofman, 1997). In addition to operating controls, the operating cycle impacts cash flow from operations (Stickney, Brown \& Wahlen, 2004, p. 116). Firm management typically perceives the value of operations by means of managerial reports based on accounting measures (Davidson \& Weil, 1978; Johnson, 1981; Palia, 2007; Roztocki, 2000a; Roztocki 2000b; Roztocki, 2000c, Roztocki, N., \& Needy, 2000; Roztocki \& Weistroffer, 2005; Roztocki \& Weistroffer, 2006) which have a long history of use (Fleischman, \& Tyson, 1997; Heier, 2000). This managerial reporting directly impacts management perception of firm value and contributes to financing and investing decisions made by management. Firm valuation typically comes from cash flows (Copeland, Weston and Shastri, 2005; Damodaran, 2002; Ruback, 2002) and managerial reporting contributes to an asymmetry of information between management perception and the market perception of firm value (Chirinko \& Singha, 2000; Fama \& French, 2002) since market perception is constrained by information contained in financial reports and the firms risk to anticipate stock returns (Fama \& French, 1992). A variety of factors influence management corporate financing decisions (Taggert, 1977) and decisions about the optimal capital structure. Major factors are: cost of debt and equity (Kemsley \& Nissim, 2002; Modigliani \& Miller, 1958), bankruptcy costs (Altman, 1984), lease versus debt (Ang \& Peterson, 1984), retention of dividends (Allen, Bernardo, \& Welch, 2000; Bell \& Jenkinson, 2002; Fama \& French, 2002; Grullon \& Michaely, 2002), reduction of risk through diversification (Billet \& Mauer, 2000), market characteristics (Baker \& Wurgler, 2002), and use of options (Black \& Scholes, 1972; Fischer, 1978). Firm management can further use finance policy as a 
form of signaling (DeAngelo, DeAngelo \& Skinner, 2000) to impact market perception of firm value, and thereby impact stock price. 


\section{Appendix B: Proposed Dissertation Timeline}

\begin{tabular}{|c|c|c|}
\hline & 2007 & $\begin{array}{c}2008 \\
131415161718192021222324\end{array}$ \\
\hline Month & 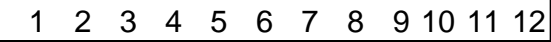 & 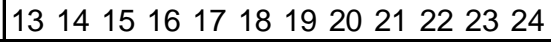 \\
\hline \multicolumn{3}{|l|}{ Dissertation Proposal } \\
\hline \multicolumn{3}{|l|}{ Literature Review } \\
\hline \multicolumn{3}{|l|}{ Research Methodology } \\
\hline \multicolumn{3}{|l|}{ Survey Development } \\
\hline \multirow{2}{*}{\multicolumn{3}{|c|}{ Define Survey Software }} \\
\hline & & \\
\hline \multicolumn{3}{|l|}{ Survey Pilot Test } \\
\hline \multirow{2}{*}{\multicolumn{3}{|c|}{$\begin{array}{l}\text { Survey Distribution } \\
\text { Survey Analysis }\end{array}$}} \\
\hline & & \\
\hline \multicolumn{3}{|l|}{ Survey Conclusions } \\
\hline \multicolumn{3}{|l|}{ Results and Discussion } \\
\hline \multicolumn{3}{|l|}{ Modifications/Defense } \\
\hline & 2009 & 2010 \\
\hline Month & 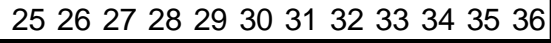 & 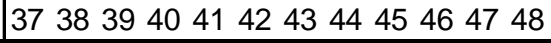 \\
\hline \multicolumn{3}{|l|}{ Dissertation Proposal } \\
\hline \multicolumn{3}{|l|}{ Literature Review } \\
\hline \multirow{2}{*}{\multicolumn{3}{|c|}{$\begin{array}{l}\text { Research Methodology } \\
\text { Survey Develooment }\end{array}$}} \\
\hline & & \\
\hline \multicolumn{3}{|l|}{ Define Survey Software } \\
\hline \multicolumn{3}{|l|}{ Define Source Database } \\
\hline \multicolumn{3}{|l|}{ Survey Pilot Test } \\
\hline \multicolumn{3}{|l|}{ Survey Distribution } \\
\hline \multirow{2}{*}{\multicolumn{3}{|c|}{$\begin{array}{l}\text { Survey Analysis } \\
\text { Survey Conclusions }\end{array}$}} \\
\hline & & \\
\hline \multicolumn{3}{|l|}{ Results and Discussion } \\
\hline \multicolumn{3}{|l|}{ Modifications/Defense } \\
\hline & $\begin{array}{c}2011 \\
495051525354555657585960\end{array}$ & $\begin{array}{c}2012 \\
616263646566676869707172\end{array}$ \\
\hline \multicolumn{3}{|l|}{ Dissertation Proposal } \\
\hline \multirow{2}{*}{\multicolumn{3}{|c|}{ Literature Review }} \\
\hline Research Methodology & & \\
\hline \multicolumn{3}{|l|}{ Survey Development } \\
\hline \multicolumn{3}{|l|}{ Define Survey Software } \\
\hline \multicolumn{3}{|l|}{ Define Source Database } \\
\hline \multirow{2}{*}{\multicolumn{3}{|c|}{$\begin{array}{l}\text { Survey Pilot Test } \\
\text { Survey Distribution }\end{array}$}} \\
\hline & & \\
\hline Survey Analysis & & \\
\hline Survey Conclusions & & \\
\hline Results and Discussion & & \\
\hline Modifications/Defense & & \\
\hline
\end{tabular}




\section{Appendix C: Hypotheses}

$\mathrm{H}_{1}$ : Salience of business processes identified for measurement correlate to firm value as tested by Kruskal-Wallis one-way ANOVA.

$\mathrm{H}_{1 \mathrm{a}}$ : Identity business processes using the means results from $\mathrm{H}_{1}$ have a higher positive correlation to firm value than Priority business processes as tested by the Chi-Square Contingency Test of Association.

$\mathrm{H}_{1 \mathrm{~b}}$ : Priority business processes using the means results from $\mathrm{H}_{1}$ have a higher positive correlation to firm value than Background business processes as tested by the Chi-Square Contingency Test of Association.

$\mathrm{H}_{2}$ : Nonfinancial performance measures are more correlated to firm value than financial measures as tested by Kruskal-Wallis one-way ANOVA.

$\mathrm{H}_{3}$ : The relative importance of nonfinancial performance measures compared to financial measures using the results from $\mathrm{H}_{2}$ is greater in high clock-speed industries than in low clock-speed industries as tested by Cramer's Phi.

$\mathrm{H}_{4}$ : The number of categories of Balanced Scorecard metrics used correlate to firm value as tested by Kruskal-Wallis one-way ANOVA. 


\section{Appendix D:}

\section{Consent Form \\ Web-Based Survey}

Performance Measures for Managerial Decision Making: Performance Measurement Synergies in Multi-Attribute Performance Measurement Systems

You are invited to participate in a research study conducted by Robert Fowke from Portland State University, Systems Science: Business Administration. The researcher hopes to learn the impact of use of different performance measures, varied feedback lags, and distribution of performance measurement information on business performance, in partial fulfillment of the requirements for a doctoral degree, under the supervision of Dr. Beverly Fuller. You were selected as a possible participant in this study based on your employment in a company included in North American Industrial Classification System (NAICS) categories under review.

If you decide to participate, you will be asked to complete the following questionnaire regarding performance measurement criteria and processes in your company/department. Any information that is obtained in connection with this study and that can be linked to you or identify you will be kept confidential. This information will be kept confidential by coding of companies and participants (Company XXXX, Participant $1-\mathrm{x}$ ). Each participant has the option to receive results of the study upon request.

Your participation is voluntary. You do not have to take part in this study, and it will not affect your relationship with your company or Portland State University. You may also withdraw from this study at any time without affecting your relationship with your company or Portland State University.

If you have concerns or problems about your participation in this study or your rights as a research subject, please contact the Human Subjects Research Review Committee, Office of Research and sponsored Projects, 111 Cramer Hall, Portland State University, (503) 725-4288 / 1-877-480-4400. If you have questions about the study itself, contact Robert Fowke at Portland State University, c/o Dr. Beverly Fuller, SBA.

By checking this box to continue indicates that you have read and understand the above information and agree to take part in this study. Please understand that you may withdraw your consent at any time without penalty, and that, by not continuing, you are not waiving any legal claims, rights or remedies. The researcher will provide you with a copy of this form for your own records upon request.

Results of the study will be available to participants upon request. 


\section{Appendix E: Questionnaire}

1) How frequently does your product change/improve?

(check most appropriate)

Check One

$<3$ yrs

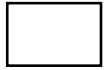

$4-10$ yrs

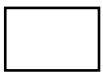

$>10 \mathrm{yrs}$

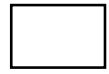

Don't know

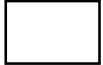

2) How frequently do your technical processes change/improve?

(check most appropriate)

$<3$ yrs

Check One

$4-10$ yrs

$>10 \mathrm{yrs}$

Don't know
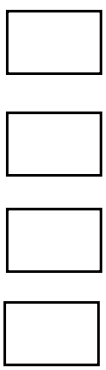

3) How frequently does your company or department change its performance measurement system?

$<3$ yrs

Check One

3 - 10 years

$>10 \mathrm{yrs}$

Don't know

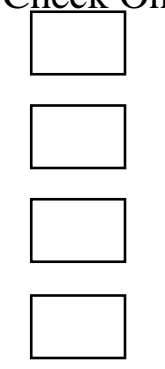


4) What is your position in the company?

Executive

Check One

VP

Front line Supervisor
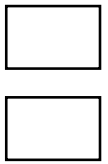

Other
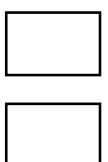

5) In which department do you work?

Corporate

Check One

HR

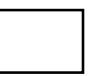

Finance

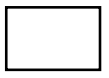

Marketing

Production

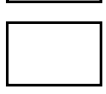

Engineering

Other
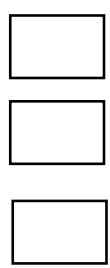

Estimate

Number

6) Estimated Number of Financial Measures you use to monitor performance, or which are used to measure your performance (see following sample list)

\begin{tabular}{|l|l|}
\hline Financial & \\
\hline Sales & Return on Investment \\
\hline Capital Expenditures & Total Manufacturing Cost \\
\hline Maintenance Expenditures & Labor cost \\
\hline Operating Expense & Material cost \\
\hline SG \& A Expense & Indirect (overhead) cost \\
\hline
\end{tabular}


7) Estimated Number of Internal Operating (Nonfinancial) Measures you use to monitor performance, or which are used to measure your performance

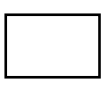
(see following sample list)

\begin{tabular}{|l|l|}
\hline \multicolumn{2}{|c|}{ Internal Operating (Nonfinancial) } \\
\hline Information Technology (\% cost) & Suppliers (Dependability, on time Delivery) \\
\hline New Product \% of Sales & Suppliers (number) \\
\hline Proprietary Products \% of Sales & R\&D (New Product Introduction Cycle Time) \\
\hline New Product Introduction vs Competitors & Production Volume \\
\hline New Product Introduction vs Plan & $\begin{array}{l}\text { Labor Productivity (hours used, available, } \\
\text { overtime) }\end{array}$ \\
\hline Manufacturing Process Capabilities & $\begin{array}{l}\text { Machine Productivity (hours running, available, } \\
\text { downtime) }\end{array}$ \\
\hline Time to Develop Next Generation of Products & Material usage (inefficiency, waste) \\
\hline Product Quality (defect rates) & Setup Efficiency (setup time, number of setups) \\
\hline Delivery (on time) & Manufacturing Cycle Time (total process time) \\
\hline Manufacturing Efficiency & Inventory (turnover) \\
\hline Suppliers (quality, defect rates) & $\begin{array}{l}\text { Product Defects (number of errors, rework, } \\
\text { scrap) }\end{array}$ \\
\hline
\end{tabular}

8) Estimated Number of Employee (Learning and Growth, Nonfinancial) Measures you use to monitor performance, or which are used to measure Estimate Number your performance (see following sample list)

\section{Employee (Learning and Growth, Nonfinancial)}

\begin{tabular}{|l|l|}
\hline Safety (number of accidents, injuries) & $\begin{array}{l}\text { Employee Training/Education (hours or time } \\
\text { allocated for training) }\end{array}$ \\
\hline Employee satisfaction (surveys, grievances) & Employee Loyalty / Turnover \\
\hline Employee Skills (level of education, experience) & Absenteeism \\
\hline
\end{tabular}


Employee Empowerment (\# suggestions, \# on improvement teams)

Estimate

Number

9) Estimated Number of Customer (Nonfinancial) Measures you use to monitor performance, or which are used to measure your performance (see following sample list)

\begin{tabular}{|l|l|}
\hline \multicolumn{2}{|c|}{ Customer (Nonfinancial) } \\
\hline $\begin{array}{l}\text { Customer Acquisition (\# new, \% Sales From } \\
\text { New) }\end{array}$ & Time to Fill Customer Orders \\
\hline $\begin{array}{l}\text { Customer Retention / Loyalty (\# Repeat } \\
\text { Customers) }\end{array}$ & $\begin{array}{l}\text { Deliver Performance (on-time, \% Correct } \\
\text { Delivery) }\end{array}$ \\
\hline Customer Satisfaction (surveys, complaints) & Time to Respond to Customer Problems \\
\hline $\begin{array}{l}\text { Phone System Utility (Automated, Response } \\
\text { Time) }\end{array}$ & $\begin{array}{l}\text { Flexibility/Responsiveness (ability to vary } \\
\text { product) }\end{array}$ \\
\hline Market Share & \\
\hline
\end{tabular}

10) Are the performance measures you use primarily related to:

(rank $1-4,1$ most related and 4 least related)

Identity processes (processes that define your company)

Rank 1 - 4

Priority processes (processes that are critical to support the identity of your company)

Background processes (processes that are necessary support to daily operations)

Mandated processes (processes necessary for regulatory compliance) 


\section{Appendix F: NAICS Company Listings}

\begin{tabular}{|c|c|c|c|c|}
\hline \multicolumn{5}{|c|}{$\begin{array}{l}\text { http://www.mergentonline.com.proxy.lib.pdx.edu/compsearchresults.asp } \\
\text { searchtype=compname\&searchtext=\&codetype=naic\&industrycode= } \\
31621 \& \text { Index=null\&country=null\&usonly=on\&bstype=codeandcountry }\end{array}$} \\
\hline \multirow{2}{*}{\multicolumn{5}{|c|}{$\begin{array}{l}\text { Prim NAICS } 31621 \\
\text { Footwear Manufacturing }\end{array}$}} \\
\hline & & & & \\
\hline \multicolumn{2}{|l|}{ US Exchanges Only } & \multicolumn{2}{|c|}{ Accessed: } & $9 / 12 / 2007$ \\
\hline Company Name & \begin{tabular}{|l|l|} 
SIC & Exchange \\
\end{tabular} & Ticker & Active/In & Country \\
\hline Barry (R.G.) Corp. & \begin{tabular}{|l|l|}
3149 & ASE \\
\end{tabular} & DFZ & Active & United States \\
\hline Brown Shoe Co., Inc. & 3144 NYS & BWS & Active & United States \\
\hline Cole (Kenneth) Productions, Inc. & 3143 NYS & KCP & Active & United States \\
\hline Crocs Inc & 3021 NMS & CROX & Active & United States \\
\hline Deckers Outdoor Corp. & 3021 NMS & DECK & Active & United States \\
\hline Foot Locker, Inc. & 5661 NYS & $\mathrm{FL}$ & Active & United States \\
\hline Iconix Brand Group Inc & 3149 NMS & ICON & Active & United States \\
\hline K-Swiss, Inc & 3149 NMS & KSWS & Active & United States \\
\hline LaCrosse Footwear, Inc. & 3021 NMS & BOOT & Active & United States \\
\hline Madden (Steven) Ltd. & 3144 NMS & $\mathrm{SHOO}$ & Active & United States \\
\hline NIKE, Inc & 3021 NYS & NKE & Active & United States \\
\hline Phoenix Footwear Group, Inc. & \begin{tabular}{|l|l|}
3144 & ASE \\
\end{tabular} & PXG & Active & United States \\
\hline Rocky Brands Inc & 3143 NMS & RCKY & Active & United States \\
\hline Skechers U S A, Inc. & 3143 NYS & SKX & Active & United States \\
\hline Skins Inc & 3149 OTC & SKNN & Active & United States \\
\hline Timberland Co. (The) & 3143 NYS & $\mathrm{TBL}$ & Active & United States \\
\hline Weyco Group, Inc & 5139 NMS & WEYS & Active & United States \\
\hline Wolverine World Wide, I & 3149 NYS & WWW & Active & United States \\
\hline
\end{tabular}




\begin{tabular}{|c|c|c|c|c|}
\hline $\begin{array}{l}\text { http://www.mergentonline.com.pro } \\
\text { compname\&searchtext=\&codetyp } \\
\text { null\&usonly=on\&bstype=codeandc }\end{array}$ & $\begin{array}{l}\text { y.lib.pdx.edu/col } \\
=\text { naic\&industryc } \\
\text { ountry }\end{array}$ & $\begin{array}{l}\text { mpsearchr } \\
\text { ode }=33121\end{array}$ & $\begin{array}{l}\text { sults.asp } \\
\text { \&lndex }\end{array}$ & $\begin{array}{l}\mathrm{p} ? \text { searchtype }= \\
=\text { null\&country= }\end{array}$ \\
\hline $\begin{array}{l}\text { Prime NAICS } 331210 \\
\text { Iron and Steel Pipe and Tube Man }\end{array}$ & dfacturing from $P$ & urchased & Steel & \\
\hline US Exchanges Only & & Accessed & & 9/12/2007 \\
\hline Company Name & \begin{tabular}{l|l} 
SIC & Exchange \\
\end{tabular} & Ticker & Active/In & Country \\
\hline Allegheny Technologies, Inc (US) & 3317 NYS & ATI & Active & United States \\
\hline Corinth Pipeworks SA (Greece) & 3317 OTC & CPWK F & Active & Greece \\
\hline Dayton Superior Corporation & 3317 NMS & DSUP & Active & United States \\
\hline Friedman Industries, Inc. (US) & \begin{tabular}{|l|l|}
3317 & ASE \\
\end{tabular} & FRD & Active & United States \\
\hline Northwest Pipe Co. & 3317 NMS & NWPX & Active & United States \\
\hline Novamerican Steel Inc. & 3317 NMS & TONS & Active & Canada \\
\hline Sumitomo Pipe \& Tube (Jpn) & 3317 OTC & SIBT F & Active & Japan \\
\hline Synalloy Corp. & 3317 NMS & SYNL & Active & United States \\
\hline Tarpon Industries Inc & 3317|ASE & TPO & Active & United States \\
\hline Tenaris SA & 3317 NYS & TS & Active & Luxembourg \\
\hline
\end{tabular}




\begin{tabular}{|c|c|c|c|c|}
\hline \multicolumn{5}{|c|}{$\begin{array}{l}\text { http://www.mergentonline.com.ezproxy.lib.pdx.edu/compsearchresults.asp?searchtype= } \\
\text { compname\&searchtext=\&codetype=naic\&industrycode=33151\&Index=null\&country= } \\
\text { null\&usonly=on\&bstype=codeandcountry }\end{array}$} \\
\hline \multicolumn{5}{|l|}{$\begin{array}{l}\text { Prim NAICS } 33151 \\
\text { Ferrous Metal Foundries }\end{array}$} \\
\hline US Exchanges Only & & Accessec & & 9/17/2007 \\
\hline Company Name & \begin{tabular}{|l|l|} 
SIC & Exchange \\
\end{tabular} & Ticker & Active/Inactive & Country \\
\hline Aceralia Corporacion Siderurgica(Spain) & 3325 OTC & ARAL Y & Inactive & Spain \\
\hline Amanasu Environment Corp & 3325 OTC & AMSU & Active & United States \\
\hline Ampco-Pittsburgh Corp. & 3325 NYS & AP & Active & United States \\
\hline Arcelor Mittal & 3325 NYS & MT & Active & Netherlands \\
\hline Atchison Casting Corp. (US) & 3325 OTC & AHNC Q & Active & United States \\
\hline Buderus AG Lahn Wetzlar & 3312 OTC & BRSJ F & Active & Germany \\
\hline Grupo Simec S.A.B. de C.V. & 3325 ASE & SIM & Active & Mexico \\
\hline Kawagishi Bridge Works Co., Ltd.(Jpn) & 3325 OTC & KGBG F & Active & Japan \\
\hline Mechel OAO & 3325 NYS & MTL & Active & Russia \\
\hline Olympic Steel Inc. & 5051 NMS & ZEUS & Active & United States \\
\hline Precision Castparts Corp. & 3324 NYS & PCP & Active & United States \\
\hline Steel Dynamics Inc. & 3312 NMS & STLD & Active & United States \\
\hline Thyssen Industrie AG (Germany, F.R.) & 3325 OTC & THYI F & Active & Germany \\
\hline United States Steel Corp. (New) & 3312 NYS & $x$ & Active & United States \\
\hline Universal Stainless \& Alloy Products & 3312 NMS & USAP & Active & United States \\
\hline
\end{tabular}




\begin{tabular}{|c|c|c|c|c|}
\hline \multicolumn{5}{|c|}{$\begin{array}{l}\text { http://www.mergentonline.com.proxy.lib.pdx.edu/compsearchre } \\
\text { searchtype=compname\&searchtext=\&codetype=naic\&industry } \\
\text { Index=null\&country=null\&usonly=on\&bstype=codeandcountry }\end{array}$} \\
\hline \multirow{2}{*}{\multicolumn{5}{|c|}{$\begin{array}{l}\text { Prim NAICS } 334111 \\
\text { Electronic Computer Manufacturing } \\
\text { US Exchanges Only }\end{array}$}} \\
\hline & & Accessed & & 9/12/2007 \\
\hline Company Name & \begin{tabular}{|l|l|} 
SIC & Exchange \\
\end{tabular} & Ticker & Active/In & Country \\
\hline Apple Inc & 3571 NMS & AAPL & Active & United States \\
\hline Catcher Holdings, Inc. & 3571 OTC & $\mathrm{CTHH}$ & Active & United States \\
\hline Cintel Corp & 3571 OTC & CNCN & Active & United States \\
\hline Concurrent Computer Corp. (US) & 3571 NMS & CCUR & Active & United States \\
\hline Cray Inc & 3571 NMS & CRAY & Active & United States \\
\hline Daewoo Telecom. (S.Korea) & 3571 OTC & DWOO F & Active & Korea (South) \\
\hline Dell Inc & 3571 NMS & DELL & Active & United States \\
\hline Gateway Inc & 3571 NYS & GTW & Active & United States \\
\hline Heiler Software AG (Ger & 3571 OTC & HEIR F & Active & Germany \\
\hline Hewlett-Packard Co & 3571 NYS & $\mathrm{HPQ}$ & Active & United States \\
\hline InPlay Technologies Inc & 3571 NAS & NPLA & Active & United States \\
\hline International Busin & 3571 NYS & IBM & Active & United States \\
\hline Maxwell Technologies, Inc. (US) & 3571 NMS & MXWL & Active & United States \\
\hline Micro Book International, Inc. (FL) & \begin{tabular}{|l|l|}
3571 & OTC \\
\end{tabular} & MBKI & Active & United States \\
\hline National Datacomputer, Inc. & 3571 OTC & IDCP & Active & United States \\
\hline NDS Group Plc & 3571 NMS & NNDS & Active & United Kingdom \\
\hline NEC Corp & 3571 NMS & NIPN Y & Active & Japan \\
\hline Neoware Inc & 3571 NMS & NWRE & Active & United States \\
\hline Omnicell Inc & 3571 NMS & OMCL & Active & United States \\
\hline Rackable Systen & 3571 NMS & RACK & Active & United States \\
\hline Sandston Corp & 3571 OTC & SDON & Active & United States \\
\hline Silicon Graphics Inc. & 3571 NAS & SGIC & Active & United States \\
\hline Socket Communications, Inc. & \begin{tabular}{|l|l|}
3571 & NMS \\
\end{tabular} & SCKT & Active & United States \\
\hline SteelCloud Inc & 3571 NAS & SCLD & Active & United States \\
\hline Sun Microsystems Inc & \begin{tabular}{|l|l|}
3571 & NMS \\
\end{tabular} & JAVA & Active & United States \\
\hline Super Micro Computer Inc & 3571 NMS & SMCI & Active & United States \\
\hline Web.com Inc & \begin{tabular}{|l|l|}
7389 & NMS \\
\end{tabular} & WWWW & Active & United States \\
\hline XATA Corp. & 3571 NAS & XATA & Active & United States \\
\hline
\end{tabular}




\begin{tabular}{|c|c|c|c|c|}
\hline \multicolumn{5}{|c|}{$\begin{array}{l}\text { http://www.mergentonline.com.ezproxy.lib.pdx.edu/compsearch } \\
\text { searchtype=compname\&searchtext=\&codetype=naic\&industry } \\
\text { Index=null\&country=null\&usonly=on\&bstype=codeandcountry }\end{array}$} \\
\hline \multirow{2}{*}{\multicolumn{5}{|c|}{$\begin{array}{l}\text { Prim NAICS } 334119 \\
\text { Other Computer Peripheral Equipment Manufacturing }\end{array}$}} \\
\hline & & & & \\
\hline \multicolumn{2}{|l|}{ US Exchanges Only } & \multicolumn{2}{|c|}{ Accessed: } & 9/17/2007 \\
\hline Company Name & \begin{tabular}{|l|l|} 
SIC & Exchange \\
\end{tabular} & Ticker & Active/Inactive & Country \\
\hline 3Com Corp. & 3577 NMS & COMS & Active & United States \\
\hline Adaptec Inc. (United States) & 3577NMS & ADPT & Active & United States \\
\hline Alliance Distributors Holding & 3577 OTC & ADTR & Active & United States \\
\hline Amedia Networks Inc & 3577 OTC & AANI & Active & United States \\
\hline Apem SA (France) & 3577 OTC & AEMS F & Active & France \\
\hline Astrocom Corp. & 3577 OTC & ATCC Q & Active & United States \\
\hline Astro-Med, Inc. & 3577 NMS & ALOT & Active & United States \\
\hline Avici Systems Inc (US) & 3577 NMS & $\mathrm{AVCl}$ & Active & United States \\
\hline Avocent Corp (United States) & 3577 NMS & AVCT & Active & United States \\
\hline Bio-Key International Inc & 3577 OTC & BKYI & Active & United States \\
\hline Black Box Corp. (DE) (US) & 3577 NMS & BBOX & Active & United States \\
\hline Communication Intelligence (DE) & 3577 OTC & $\mathrm{CICl}$ & Active & United States \\
\hline Copytele Inc & 3577 OTC & COPY & Active & United States \\
\hline CSP Inc & 3577 NMS & CSPI & Active & United States \\
\hline DataMetrics Corp. & 3577 OTC & DMCP & Active & United States \\
\hline Digi International, Inc. (US) & 3577 NMS & DGII & Active & United States \\
\hline Digital River, Inc. & 5045 NMS & DRIV & Active & United States \\
\hline Emulex Corporation (US) & 3577 NYS & ELX & Active & United States \\
\hline Extreme Networks, Inc. & 3577 NMS & EXTR & Active & United States \\
\hline Ezenia! Inc. (United States) & 3577 OTC & EZEN & Active & United States \\
\hline F5 Networks, Inc. & 3577 NMS & FFIV & Active & United States \\
\hline FiberTower Corp & \begin{tabular}{|l|l|} 
NMI2 \\
\end{tabular} & FTWR & Active & United States \\
\hline Foundry Networks Inc & 3577 NMS & FDRY & Active & United States \\
\hline Franklin Wireless Corp & 7373 OTC & FKLT & Active & United States \\
\hline Hauppauge Digital, Inc. & 3577 NMS & HAUP & Active & United States \\
\hline Hypercom Corp & 3578 NYS & HYC & Active & United States \\
\hline icad inc (United States) & 3577 NAS & ICAD & Active & United States \\
\hline Immersion Corp (United States) & 3577 NMS & IMMR & Active & United States \\
\hline InFocus Corp & 3577 NMS & INFS & Active & United States \\
\hline Intelligent Systems Corp. & 8742 ASE & INS & Active & United States \\
\hline International Lottery \& Totalizator Sys & 3578 OTC & ITSI & Active & United States \\
\hline Interphase Corp. (United States) & 3577 NMS & INPH & Active & United States \\
\hline Intrusion Inc & 3577 OTC & INTZ & Active & United States \\
\hline Ion Networks, Inc. & 3577 OTC & IONN & Active & United States \\
\hline Juniper Networks Inc & 3577 NMS & JNPR & Active & United States \\
\hline Key Tronic Corp. & 3577 NMS & KTCC & Active & United States \\
\hline
\end{tabular}




\begin{tabular}{|c|c|c|c|c|}
\hline Konami Corp & 3577 NYS & KNM & Active & Japan \\
\hline Lafe Technology Ltd. (Singapore) & 3679 OTC & LAFE F & Active & Bermuda \\
\hline Lantronix Inc. (United States) & 3577 NAS & LTRX & Active & United States \\
\hline Lexmark International, Inc. & 3577NYS & LXK & Active & United States \\
\hline Logitech International SA & 3577 NMS & LOGI & Active & Switzerland \\
\hline Media Sciences International Inc. & 3577 NAS & MSII & Active & United States \\
\hline Microfield Group Inc & 1731 OTC & MICG & Active & United States \\
\hline Mitek Systems, Inc. & 3577 OTC & MITK & Active & United States \\
\hline Mobility Electronics Inc (US) & 3577 NMS & MOBE & Active & United States \\
\hline Moneyflow Systems International Inc & 3578 OTC & MFLW & Active & United States \\
\hline NCR Corp. (New) & 3578 NYS & NCR & Active & United States \\
\hline Neonode Inc & 3577NAS & NEON & Active & United States \\
\hline Network Connection, Inc. (The) (US) & $3577 \mathrm{BSE}$ & NWC & Active & United States \\
\hline Orbotech Ltd. (Israel) & 3577 NMS & ORBK & Active & Israel \\
\hline Peerless Systems Corp. & 7372NAS & PRLS & Active & United States \\
\hline Printronix, Inc. & 3577 NMS & PTNX & Active & United States \\
\hline Rada Electronic Industries Ltd. & 3577NAS & RADA & Active & Israel \\
\hline RadiSys Corp. & 3577 NMS & RSYS & Active & United States \\
\hline Ridgefield Acquisition Corp & 3577 OTC & RDGA & Active & United States \\
\hline Rimage Corp. & 3577 NMS & RIMG & Active & United States \\
\hline Riverbed Technology Inc & 3577 NMS & RVBD & Active & United States \\
\hline S\&T Sys. Int. \& Tech. Dist.(Austria) & 3577 OTC & STSQ Y & Active & Austria \\
\hline SCM Microsystems, Inc. & 3577NMS & SCMM & Active & United States \\
\hline Secure Computing Corp. (US) & 3577 NMS & SCUR & Active & United States \\
\hline Sedona Corp & 5045 OTC & SDNA & Active & United States \\
\hline Sigma Designs, Inc. (United States) & 3577 NMS & SIGM & Active & United States \\
\hline Small Cap Strategies Inc & 3577 OTC & SMCA & Active & United States \\
\hline SMART Modular Technologies, Inc & 3577 NMS & SMOD & Active & Cayman Islands \\
\hline Stratasys, Inc. & 3577 NMS & SSYS & Active & United States \\
\hline TAT Technologies Ltd. (Israel) & 3577 NAS & TATT F & Active & Israel \\
\hline TransAct Technologies Inc. & 3577NMS & TACT & Active & United States \\
\hline Viseon Inc & 3577 OTC & VSNI & Active & United States \\
\hline Zamba Corp. (United States) & 5045 OTC & ZMBA & Active & United States \\
\hline
\end{tabular}




\section{Appendix G: NAICS Company Comparisons}

\begin{tabular}{|c|c|c|c|c|c|}
\hline & \multicolumn{5}{|c|}{$\begin{array}{l}\text { Prim NAICS } 31621 \\
\text { Footwear Manufacturing } \\
\text { US Exchanges Only } \\
12 \text { month rolling average year to year adjusted stock price comparison } \\
(\% \text { change }=(\mathrm{A} / \mathrm{B}-1) \%)\end{array}$} \\
\hline & $A$ & 9/07 - & $9 / 07-$ & 9/07 - & $\begin{array}{l}9 / 07- \\
\end{array}$ \\
\hline & $\mathrm{B}$ & $9 / 06$ & $9 / 05$ & $9 / 04$ & $9 / 02$ \\
\hline & Company & $1 \mathrm{yr}$ & $2 \mathrm{yr}$ & $3 \mathrm{yr}$ & $5 \mathrm{yr}$ \\
\hline 1 & Barry (R.G.) Corp. & $41 \%$ & $105 \%$ & $197 \%$ & $71 \%$ \\
\hline 2 & Brown Shoe Co., Inc. & $32 \%$ & $92 \%$ & $82 \%$ & $249 \%$ \\
\hline 3 & Cole (Kenneth) Productions, Inc. & $-4 \%$ & $-14 \%$ & $-19 \%$ & $24 \%$ \\
\hline 4 & Crocs Inc & & & & \\
\hline 5 & Deckers Outdoor Corp. & $118 \%$ & $136 \%$ & $208 \%$ & $1563 \%$ \\
\hline 6 & Foot Locker, Inc. & $-8 \%$ & $-15 \%$ & $-5 \%$ & $59 \%$ \\
\hline 7 & Iconix Brand Group Inc & $50 \%$ & $22 \%$ & $701 \%$ & $652 \%$ \\
\hline 8 & K-Swiss, Inc & $-3 \%$ & $-5 \%$ & $32 \%$ & $195 \%$ \\
\hline 9 & LaCrosse Footwear, Inc. & $33 \%$ & $45 \%$ & $113 \%$ & $425 \%$ \\
\hline 10 & Madden (Steven) Ltd. & $16 \%$ & $158 \%$ & $152 \%$ & $213 \%$ \\
\hline 11 & NIKE, Inc & $30 \%$ & $31 \%$ & $53 \%$ & $114 \%$ \\
\hline 12 & Phoenix Footwear Group, Inc. & $-26 \%$ & $-38 \%$ & $-57 \%$ & \\
\hline 13 & Rocky Brands Inc & $-35 \%$ & $-52 \%$ & $-36 \%$ & $124 \%$ \\
\hline 14 & Skechers U S A, Inc. & $38 \%$ & $107 \%$ & $160 \%$ & $79 \%$ \\
\hline 15 & Skins Inc & & & & \\
\hline 16 & Timberland Co. (The) & $-14 \%$ & $-22 \%$ & $-8 \%$ & $43 \%$ \\
\hline 17 & Weyco Group, Inc & $25 \%$ & $30 \%$ & $63 \%$ & $168 \%$ \\
\hline 18 & Wolverine World Wide, Inc. (US) & $20 \%$ & $34 \%$ & $86 \%$ & $180 \%$ \\
\hline & Count & 16 & 16 & 16 & 15 \\
\hline & Median & $22 \%$ & $30 \%$ & $73 \%$ & $168 \%$ \\
\hline & Mean & $20 \%$ & $39 \%$ & $108 \%$ & $277 \%$ \\
\hline & Standard Deviation & $36 \%$ & $64 \%$ & $178 \%$ & $391 \%$ \\
\hline & Standard Dev & on from & Mean & & \\
\hline & Barry (R.G.) Corp. & 0.60 & 1.04 & 0.50 & -0.53 \\
\hline & Brown Shoe Co., Inc. & 0.33 & 0.84 & -0.14 & -0.07 \\
\hline & Cole (Kenneth) Productions, Inc. & -0.63 & -0.81 & -0.71 & -0.65 \\
\hline & Crocs Inc & & & & \\
\hline & Deckers Outdoor Corp. & 2.71 & 1.53 & 0.56 & 3.28 \\
\hline & Foot Locker, Inc. & -0.76 & -0.83 & -0.63 & -0.56 \\
\hline & Iconix Brand Group Inc & 0.83 & -0.26 & 3.33 & 0.96 \\
\hline & K-Swiss, Inc & -0.61 & -0.68 & -0.42 & -0.21 \\
\hline & LaCrosse Footwear, Inc. & 0.37 & 0.10 & 0.03 & 0.38 \\
\hline & Madden (Steven) Ltd. & -0.09 & 1.87 & 0.25 & -0.17 \\
\hline & NIKE, Inc & 0.28 & -0.12 & -0.30 & -0.42 \\
\hline & Phoenix Footwear Group, Inc. & -1.25 & -1.19 & -0.93 & \\
\hline
\end{tabular}




\begin{tabular}{|l|r|r|r|r|} 
Rocky Brands Inc & -1.50 & -1.41 & -0.80 & -0.39 \\
\hline Skechers U S A, Inc. & 0.50 & 1.07 & 0.29 & -0.51 \\
\hline Skins Inc & & & & \\
\hline Timberland Co. (The) & -0.92 & -0.95 & -0.65 & -0.60 \\
\hline Weyco Group, Inc & 0.14 & -0.13 & -0.25 & -0.28 \\
\hline Wolverine World Wide, Inc. (US) & 0.02 & -0.07 & -0.12 & -0.25 \\
\hline \hline
\end{tabular}




\begin{tabular}{|c|c|c|c|c|c|}
\hline & $\begin{array}{l}\text { Prime NAICS } 331210 \\
\text { Iron and Steel Pipe and Tube Man } \\
\text { Steel } \\
\text { US Exchanges Only } \\
12 \text { month rolling average year to ye } \\
(\% \text { change }=(\mathrm{A} / \mathrm{B}-1) \%)\end{array}$ & acturing $f$ & stock pri & sed & \\
\hline & A & $9 / 07-$ & 9/07 - & $9 / 07-$ & $9 / 07-$ \\
\hline & $\mathrm{B}$ & $9 / 06$ & $9 / 05$ & $9 / 04$ & $9 / 02$ \\
\hline & Company & $1 \mathrm{yr}$ & $2 \mathrm{yr}$ & $3 \mathrm{yr}$ & $5 \mathrm{yr}$ \\
\hline 1 & Allegheny Technologies, Inc (US) & $86 \%$ & $324 \%$ & $667 \%$ & $691 \%$ \\
\hline 2 & Corinth Pipeworks SA (Greece) & & & & \\
\hline 3 & Dayton Superior Corporation & & & & \\
\hline 4 & Friedman Industries, Inc. (US) & $25 \%$ & $30 \%$ & $163 \%$ & $336 \%$ \\
\hline 5 & Northwest Pipe Co. & $29 \%$ & $50 \%$ & $131 \%$ & $105 \%$ \\
\hline 6 & Novamerican Steel Inc. & $12 \%$ & $1 \%$ & $145 \%$ & $606 \%$ \\
\hline 7 & Sumitomo Pipe \& Tube (Jpn) & & & & \\
\hline 8 & Synalloy Corp. & $93 \%$ & $132 \%$ & $199 \%$ & $573 \%$ \\
\hline 9 & Tarpon Industries Inc & $-71 \%$ & & & \\
\hline 10 & Tenaris SA & $42 \%$ & $236 \%$ & $650 \%$ & \\
\hline & Count & 7 & 6 & 6 & 5 \\
\hline & Median & $29 \%$ & $91 \%$ & $181 \%$ & $573 \%$ \\
\hline & Mean & $31 \%$ & $129 \%$ & $326 \%$ & $462 \%$ \\
\hline & Standard Deviation & $54 \%$ & $128 \%$ & $259 \%$ & $239 \%$ \\
\hline & Standard Devi & ion from & lean & & \\
\hline & Allegheny Technologies, Inc (US) & 1.02 & 1.53 & 1.32 & 0.96 \\
\hline & Corinth Pipeworks SA (Greece) & & & & \\
\hline & Dayton Superior Corporation & & & & \\
\hline & Friedman Industries, Inc. (US) & -0.11 & -0.77 & -0.63 & -0.53 \\
\hline & Northwest Pipe Co. & -0.03 & -0.61 & -0.75 & -1.49 \\
\hline & Novamerican Steel Inc. & -0.34 & -1.00 & -0.70 & 0.60 \\
\hline & Sumitomo Pipe \& Tube (Jpn) & & & & \\
\hline & Synalloy Corp. & 1.14 & 0.03 & -0.49 & 0.46 \\
\hline & Tarpon Industries Inc & -1.87 & & & \\
\hline & Tenaris SA & 0.20 & 0.83 & 1.25 & \\
\hline
\end{tabular}




\begin{tabular}{|c|c|c|c|c|c|}
\hline & \multicolumn{5}{|c|}{$\begin{array}{l}\text { Prim NAICS } 33151 \\
\text { Ferrous Metal Foundries } \\
\text { US Exchanges Only } \\
12 \text { month rolling average year to year adjusted stock price comparison } \\
(\% \text { change }=(\mathrm{A} / \mathrm{B}-1) \%)\end{array}$} \\
\hline & $A$ & $9 / 07-$ & $9 / 07-$ & 9/07- & $9 / 07-$ \\
\hline & $\mathrm{B}$ & $9 / 06$ & $9 / 05$ & $9 / 04$ & $9 / 02$ \\
\hline & Company & $1 \mathrm{yr}$ & $2 \mathrm{yr}$ & $3 \mathrm{yr}$ & $5 \mathrm{yr}$ \\
\hline 1 & $\begin{array}{l}\text { Aceralia Corporacion } \\
\text { Siderurgica(Spain) }\end{array}$ & & & & \\
\hline 2 & Amanasu Environment Corp & & & & \\
\hline 3 & Ampco-Pittsburgh Corp. & $51 \%$ & $176 \%$ & $195 \%$ & $271 \%$ \\
\hline 4 & Arcelor Mittal & $70 \%$ & $76 \%$ & $327 \%$ & $2669 \%$ \\
\hline 5 & Atchison Casting Corp. (US) & & & & \\
\hline 6 & Buderus AG Lahn Wetzlar & & & & \\
\hline 7 & Grupo Simec S.A.B. de C.V. & $84 \%$ & $160 \%$ & $404 \%$ & $989 \%$ \\
\hline 8 & $\begin{array}{l}\text { Kawagishi Bridge Works Co., } \\
\text { Ltd.(Jpn) }\end{array}$ & & & & \\
\hline 9 & Mechel OAO & $40 \%$ & & & \\
\hline 10 & Olympic Steel Inc. & $-2 \%$ & $43 \%$ & $107 \%$ & $549 \%$ \\
\hline 11 & Precision Castparts Corp. & $86 \%$ & $168 \%$ & $332 \%$ & $631 \%$ \\
\hline 12 & Steel Dynamics Inc. & $62 \%$ & $142 \%$ & $215 \%$ & $484 \%$ \\
\hline 13 & $\begin{array}{l}\text { Thyssen Industrie AG (Germany, } \\
\text { F.R.) }\end{array}$ & & & & \\
\hline 14 & United States Steel Corp. (New) & $60 \%$ & $103 \%$ & $182 \%$ & $459 \%$ \\
\hline 15 & Universal Stainless \& Alloy Products & $70 \%$ & $157 \%$ & $256 \%$ & $289 \%$ \\
\hline & Count & 9 & 8 & 8 & 8 \\
\hline & Median & $62 \%$ & $149 \%$ & $236 \%$ & $516 \%$ \\
\hline & Mean & $58 \%$ & $128 \%$ & $252 \%$ & $793 \%$ \\
\hline & Standard Deviation & $27 \%$ & $48 \%$ & $97 \%$ & $791 \%$ \\
\hline & Standard Devi & from $M$ & & & \\
\hline & $\begin{array}{l}\text { Aceralia Corporacion } \\
\text { Siderurgica(Spain) }\end{array}$ & & & & \\
\hline & Amanasu Environment Corp & & & & \\
\hline & Ampco-Pittsburgh Corp. & -0.24 & 0.98 & -0.59 & -0.66 \\
\hline & Arcelor Mittal & 0.46 & -1.08 & 0.77 & 2.37 \\
\hline & Atchison Casting Corp. (US) & & & & \\
\hline & Buderus AG Lahn Wetzlar & & & & \\
\hline & Grupo Simec S.A.B. de C.V. & 0.96 & 0.66 & 1.57 & 0.25 \\
\hline & $\begin{array}{l}\text { Kawagishi Bridge Works Co., } \\
\text { Ltd.(Jpn) }\end{array}$ & & & & \\
\hline & Mechel OAO & -0.66 & & & \\
\hline & Olympic Steel Inc. & -2.25 & -1.75 & -1.50 & -0.31 \\
\hline & Precision Castparts Corp. & 1.04 & 0.83 & 0.83 & -0.20 \\
\hline & Steel Dynamics Inc. & 0.16 & 0.29 & -0.38 & -0.39 \\
\hline & Thyssen Industrie AG (Germany, & & & & \\
\hline
\end{tabular}




\begin{tabular}{|l|r|r|r|r|}
\hline F.R.) & & & & \\
\hline United States Steel Corp. (New) & 0.08 & -0.51 & -0.73 & -0.42 \\
\hline Universal Stainless \& Alloy Products & 0.45 & 0.59 & 0.04 & -0.64 \\
\hline
\end{tabular}




\begin{tabular}{|c|c|c|c|c|c|}
\hline & \multicolumn{5}{|c|}{$\begin{array}{l}\text { Prim NAICS } 334111 \\
\text { Electronic Computer } \\
\text { Manufacturing } \\
\text { US Exchanges Only } \\
12 \text { month rolling average year to year adjusted stock price comparison } \\
(\% \text { change }=(\mathrm{A} / \mathrm{B}-1) \%)\end{array}$} \\
\hline & A & $9 / 07-$ & $9 / 07-$ & $9 / 07-$ & $9 / 07-$ \\
\hline & $\mathrm{B}$ & $9 / 06$ & $9 / 05$ & $9 / 04$ & $9 / 02$ \\
\hline & Company & $1 \mathrm{yr}$ & $2 \mathrm{yr}$ & $3 \mathrm{yr}$ & $5 \mathrm{yr}$ \\
\hline 1 & Apple Inc & $58 \%$ & $169 \%$ & $669 \%$ & $956 \%$ \\
\hline 2 & Catcher Holdings, Inc. & $-57 \%$ & & & \\
\hline 3 & Cintel Corp & $77 \%$ & $646 \%$ & $-26 \%$ & \\
\hline 4 & Concurrent Computer Corp. (US) & $-30 \%$ & $-21 \%$ & $-47 \%$ & $-81 \%$ \\
\hline 5 & Cray Inc & $18 \%$ & $1 \%$ & $-65 \%$ & $-16 \%$ \\
\hline 6 & Daewoo Telecom. (S.Korea) & & & & \\
\hline 7 & Dell Inc & $-4 \%$ & $-33 \%$ & $-25 \%$ & $0 \%$ \\
\hline 8 & Gateway Inc & $-16 \%$ & $-57 \%$ & $-60 \%$ & $-65 \%$ \\
\hline 9 & Heiler Software AG (Germany) & & & & \\
\hline 10 & Hewlett-Packard Co & $36 \%$ & $96 \%$ & $111 \%$ & $165 \%$ \\
\hline 11 & InPlay Technologies Inc & $-31 \%$ & $-10 \%$ & $-26 \%$ & $-75 \%$ \\
\hline 12 & International Business Machines & $27 \%$ & $22 \%$ & $17 \%$ & $17 \%$ \\
\hline 13 & Maxwell Technologies, Inc. (US) & $-23 \%$ & $22 \%$ & $27 \%$ & $57 \%$ \\
\hline 14 & $\begin{array}{l}\text { Micro Book International, Inc. } \\
\text { (FL) }\end{array}$ & & & & \\
\hline 15 & National Datacomputer, Inc. & $-53 \%$ & $-69 \%$ & $-76 \%$ & $-56 \%$ \\
\hline 16 & NDS Group Plc & $6 \%$ & $44 \%$ & $99 \%$ & $216 \%$ \\
\hline 17 & NEC Corp & $-16 \%$ & $-9 \%$ & $-30 \%$ & $-31 \%$ \\
\hline 18 & Neoware Inc & $-35 \%$ & $23 \%$ & $23 \%$ & $43 \%$ \\
\hline 19 & Omnicell Inc & $62 \%$ & $151 \%$ & $41 \%$ & $207 \%$ \\
\hline 20 & Rackable Systems Inc & $-42 \%$ & & & \\
\hline 21 & Sandston Corp & $117 \%$ & $15 \%$ & $-79 \%$ & $-90 \%$ \\
\hline 22 & Silicon Graphics Inc. & & & & \\
\hline 23 & Socket Communications, Inc. & $-21 \%$ & $-32 \%$ & $-68 \%$ & $-32 \%$ \\
\hline 24 & SteelCloud Inc & $-18 \%$ & $-53 \%$ & $-65 \%$ & $-47 \%$ \\
\hline 25 & Sun Microsystems Inc & $24 \%$ & $32 \%$ & $29 \%$ & $-30 \%$ \\
\hline 26 & Super Micro Computer Inc & & & & \\
\hline 27 & Web.com Inc & $2 \%$ & $97 \%$ & $10 \%$ & $-79 \%$ \\
\hline 28 & XATA Corp. & $-9 \%$ & $-8 \%$ & $-8 \%$ & $4 \%$ \\
\hline & Count & 23 & 21 & 21 & 20 \\
\hline & Median & $-9 \%$ & $15 \%$ & $-25 \%$ & $-23 \%$ \\
\hline & Mean & $3 \%$ & $49 \%$ & $21 \%$ & $53 \%$ \\
\hline & Standard Deviation & $44 \%$ & $151 \%$ & $158 \%$ & $232 \%$ \\
\hline & Standard De & n from & lean & & \\
\hline & Apple Inc & 1.25 & 0.80 & 4.10 & 3.90 \\
\hline & Catcher Holdings, Inc. & -1.37 & & & \\
\hline
\end{tabular}




\begin{tabular}{|l|r|r|r|r|}
\hline Cintel Corp & 1.68 & 3.96 & -0.30 & \\
\hline Concurrent Computer Corp. (US) & -0.75 & -0.46 & -0.43 & -0.58 \\
\hline Cray Inc & 0.34 & -0.32 & -0.55 & -0.30 \\
\hline Daewoo Telecom. (S.Korea) & & & & \\
\hline Dell Inc & -0.16 & -0.54 & -0.30 & -0.23 \\
\hline Gateway Inc & -0.44 & -0.70 & -0.52 & -0.51 \\
\hline Heiler Software AG (Germany) & & & & \\
\hline Hewlett-Packard Co & 0.75 & 0.31 & 0.57 & 0.48 \\
\hline InPlay Technologies Inc & -0.78 & -0.39 & -0.30 & -0.55 \\
\hline International Business Machines & 0.55 & -0.18 & -0.03 & -0.15 \\
\hline Maxwell Technologies, Inc. (US) & -0.61 & -0.18 & 0.04 & 0.01 \\
\hline Micro Book International, Inc. & & & & \\
(FL) & & & & \\
\hline National Datacomputer, Inc. & -1.29 & -0.78 & -0.62 & -0.47 \\
\hline NDS Group Plc & 0.06 & -0.03 & 0.49 & 0.70 \\
\hline NEC Corp & -0.43 & -0.39 & -0.33 & -0.36 \\
\hline Neoware Inc & -0.87 & -0.17 & 0.01 & -0.04 \\
\hline Omnicell Inc & 1.34 & 0.68 & 0.12 & 0.67 \\
\hline Rackable Systems Inc & -1.02 & & & \\
\hline Sandston Corp & 2.60 & -0.23 & -0.64 & -0.62 \\
\hline Silicon Graphics Inc. & & & & \\
\hline Socket Communications, Inc. & -0.55 & -0.54 & -0.57 & -0.37 \\
\hline SteelCloud Inc & -0.49 & -0.67 & -0.55 & -0.43 \\
\hline Sun Microsystems Inc & 0.48 & -0.11 & 0.05 & -0.36 \\
\hline Super Micro Computer Inc & & & & \\
\hline Web.com Inc & -0.02 & 0.32 & -0.07 & -0.57 \\
\hline XATA Corp. & -0.27 & -0.38 & -0.18 & -0.21 \\
\hline \hline & & & & \\
\hline
\end{tabular}




\begin{tabular}{|c|c|c|c|c|c|}
\hline & \multicolumn{5}{|c|}{$\begin{array}{l}\text { Prime NAICS } 334413 \\
\text { Semiconductor and Related Device Manufacturing } \\
\text { US Exchanges Only } \\
12 \text { month rolling average year to year adjusted stock price comparison } \\
(\% \text { change }=(\mathrm{A} / \mathrm{B}-1) \%)\end{array}$} \\
\hline & $A$ & $9 / 07-$ & $9 / 07-$ & $9 / 07-$ & $9 / 07-$ \\
\hline & $\mathrm{B}$ & $9 / 06$ & $9 / 05$ & $9 / 04$ & $9 / 02$ \\
\hline & Company & $1 \mathrm{yr}$ & $2 \mathrm{yr}$ & $3 \mathrm{yr}$ & $5 \mathrm{yr}$ \\
\hline 1 & Actel Corp. & $3 \%$ & $-2 \%$ & $-29 \%$ & $-20 \%$ \\
\hline 2 & Actielec Technologies (France) & & & & \\
\hline 3 & Actions Semiconductor Co Ltd & & & & \\
\hline 4 & Advanced Analogic Technologies & $-32 \%$ & & & \\
\hline 5 & Advanced Micro Devices, Inc. & $-46 \%$ & $-15 \%$ & $7 \%$ & $37 \%$ \\
\hline 6 & Advanced Photonix, Inc. & & & & \\
\hline 7 & Advanced Semiconducter Eng. & $30 \%$ & $81 \%$ & $54 \%$ & $108 \%$ \\
\hline 8 & AKN Technology BHD & & & & \\
\hline 9 & Alliance Fiber Optic Products (US) & $37 \%$ & $89 \%$ & $40 \%$ & $100 \%$ \\
\hline 10 & Altera Corp. (United States) & $13 \%$ & $3 \%$ & $1 \%$ & $21 \%$ \\
\hline 11 & AMIS Holdings Inc & $11 \%$ & $-15 \%$ & $-34 \%$ & \\
\hline 12 & Amkor Technology Inc. & & & & \\
\hline 13 & ANADIGICS, Inc. & $82 \%$ & $380 \%$ & $125 \%$ & $22 \%$ \\
\hline 14 & Analog Devices, Inc. & $2 \%$ & $-2 \%$ & $-18 \%$ & $4 \%$ \\
\hline 15 & Apogee Technology, Inc. & $-2 \%$ & $-61 \%$ & $-90 \%$ & $-84 \%$ \\
\hline 16 & Applied Materials, Inc. (US) & $12 \%$ & $17 \%$ & $-2 \%$ & $1 \%$ \\
\hline 17 & Applied Micro Circuits Corp. & $5 \%$ & $-1 \%$ & $-40 \%$ & $-58 \%$ \\
\hline 18 & ASAT Holdings Ltd & $-46 \%$ & $-60 \%$ & $-82 \%$ & $-72 \%$ \\
\hline 19 & Ascent Solar Technologies Inc & & & & \\
\hline 20 & ASE Test Ltd. (Singapore) & $44 \%$ & $101 \%$ & $23 \%$ & $14 \%$ \\
\hline 21 & ASM International N.V. & $42 \%$ & $54 \%$ & $24 \%$ & $37 \%$ \\
\hline 22 & Atheros Communications Inc & $46 \%$ & $154 \%$ & & \\
\hline 23 & Atmel Corp. & $21 \%$ & $94 \%$ & $-4 \%$ & $-16 \%$ \\
\hline 24 & ATMI, Inc. & $9 \%$ & $17 \%$ & $31 \%$ & $33 \%$ \\
\hline 25 & AuthenTec Inc & & & & \\
\hline 26 & Avanex Corp & $13 \%$ & $6 \%$ & $-55 \%$ & $-50 \%$ \\
\hline 27 & AXT Inc & $57 \%$ & $237 \%$ & $85 \%$ & $-51 \%$ \\
\hline 28 & Broadcom Corp. & $-6 \%$ & $39 \%$ & $33 \%$ & $67 \%$ \\
\hline 29 & Brooks Automation Inc (New) & $18 \%$ & $3 \%$ & $-21 \%$ & $-51 \%$ \\
\hline 30 & Cabot Microelectronics Corp & $12 \%$ & $9 \%$ & $-14 \%$ & $-37 \%$ \\
\hline 31 & Cambridge Display Technology & $-7 \%$ & & & \\
\hline 32 & Catalyst Semiconductor, Inc. & $-6 \%$ & $-18 \%$ & $-43 \%$ & $50 \%$ \\
\hline 33 & Cavium Networks Inc & & & & \\
\hline 34 & Ceva Inc & $25 \%$ & $8 \%$ & $-12 \%$ & \\
\hline 35 & Chartered Semiconductor Mfg. Ltd. & $2 \%$ & $27 \%$ & $-1 \%$ & $-58 \%$ \\
\hline 36 & ChipMOS TECH. Bermuda & $5 \%$ & $6 \%$ & $-17 \%$ & \\
\hline 37 & Cirrus Logic, Inc. & $-3 \%$ & $32 \%$ & $9 \%$ & $-33 \%$ \\
\hline
\end{tabular}




\begin{tabular}{|c|c|c|c|c|c|}
\hline 38 & Conexant Systems Inc. & $-38 \%$ & $-4 \%$ & $-64 \%$ & $-29 \%$ \\
\hline 39 & Cree, Inc. & $-14 \%$ & $-23 \%$ & $-2 \%$ & $33 \%$ \\
\hline 40 & Cypress Semiconductor Corp. (US) & $33 \%$ & $66 \%$ & $25 \%$ & $19 \%$ \\
\hline 41 & DayStar Technologies Inc & $-54 \%$ & $-35 \%$ & & \\
\hline 42 & Diodes, Inc. & $11 \%$ & $102 \%$ & $180 \%$ & $1078 \%$ \\
\hline 43 & DPAC Technologies Corp. & $-6 \%$ & $-76 \%$ & $-91 \%$ & $-96 \%$ \\
\hline 44 & DSP Group, Inc. (United States) & $-23 \%$ & $-17 \%$ & $-18 \%$ & $11 \%$ \\
\hline 45 & eMagin Corp (DE) & $-76 \%$ & $-88 \%$ & $-93 \%$ & $-86 \%$ \\
\hline 46 & EMCORE Corp. (United States) & $-25 \%$ & $63 \%$ & $53 \%$ & $-25 \%$ \\
\hline 47 & Epcos AG & $40 \%$ & $41 \%$ & $-7 \%$ & $-45 \%$ \\
\hline 48 & ESS Technology, Inc. & $-53 \%$ & $-75 \%$ & $-90 \%$ & $-92 \%$ \\
\hline 49 & Evergreen Solar Inc. & $-25 \%$ & $54 \%$ & $249 \%$ & $289 \%$ \\
\hline 50 & Exar Corp. & $1 \%$ & $-7 \%$ & $-18 \%$ & $-30 \%$ \\
\hline 51 & Fairchild Semiconductor Int. & $-1 \%$ & $17 \%$ & $-13 \%$ & $-21 \%$ \\
\hline 52 & Finisar Corp (United States) & $9 \%$ & $147 \%$ & $47 \%$ & $-40 \%$ \\
\hline 53 & First Solar Inc & & & & \\
\hline 54 & Focus Enhancements, Inc. & $43 \%$ & $30 \%$ & $-32 \%$ & $-9 \%$ \\
\hline 55 & Formfactor Inc & $11 \%$ & $67 \%$ & $100 \%$ & \\
\hline 56 & Genesis Microchip Inc (DE) & $-45 \%$ & $-50 \%$ & $-43 \%$ & $-70 \%$ \\
\hline 57 & GSI Technology Inc & & & & \\
\hline 58 & HEI Inc & $-57 \%$ & $-57 \%$ & $-55 \%$ & $-81 \%$ \\
\hline 59 & $\mathrm{Hi} /$ fn Inc. & $5 \%$ & $-11 \%$ & $-41 \%$ & $-38 \%$ \\
\hline 60 & Himax Technologies Inc & & & & \\
\hline 61 & Hittite Microwave Corp & $19 \%$ & & & \\
\hline 62 & Hologram Ind.Marne La Vallee (Fr.) & & & & \\
\hline 63 & Ibis Technology Corp. & $-48 \%$ & $-32 \%$ & $-84 \%$ & $-82 \%$ \\
\hline 64 & Integrated Device Technology, Inc. & $9 \%$ & $36 \%$ & $7 \%$ & $-34 \%$ \\
\hline 65 & Integrated Silicon Solution, Inc. & $-4 \%$ & $-20 \%$ & $-56 \%$ & $-39 \%$ \\
\hline 66 & Intel Corp & $8 \%$ & $-6 \%$ & $-17 \%$ & $-9 \%$ \\
\hline 67 & International Rectifier Corp. & $1 \%$ & $-16 \%$ & $-15 \%$ & $10 \%$ \\
\hline 68 & Intersil Corp. & $9 \%$ & $59 \%$ & $31 \%$ & $13 \%$ \\
\hline 69 & IPG Photonics Corp & & & & \\
\hline 70 & Irvine Sensors Corp. & $-33 \%$ & $-35 \%$ & $-43 \%$ & $1 \%$ \\
\hline 71 & Isonics Corp. & $-70 \%$ & $-88 \%$ & $-68 \%$ & $-61 \%$ \\
\hline 72 & IXYS Corp. & $-4 \%$ & $-10 \%$ & $13 \%$ & $30 \%$ \\
\hline 73 & JA Solar Holdings Co Ltd & & & & \\
\hline 74 & Jazz Technologies Inc & & & & \\
\hline 75 & JDS Uniphase Corp & $-30 \%$ & $-8 \%$ & $-48 \%$ & $-63 \%$ \\
\hline 76 & Kulicke \& Soffa Industries, Inc. & $6 \%$ & $27 \%$ & $-19 \%$ & $-32 \%$ \\
\hline 77 & Lattice Semiconductor Corp. & $0 \%$ & $17 \%$ & $-27 \%$ & $-58 \%$ \\
\hline 78 & Leadis Technology Inc & $-24 \%$ & $-47 \%$ & & \\
\hline 79 & LightPath Technologies, Inc. & $26 \%$ & $52 \%$ & $8 \%$ & $-65 \%$ \\
\hline 80 & Linear Technology Corp. (US) & $-1 \%$ & $-8 \%$ & $-11 \%$ & $1 \%$ \\
\hline 81 & Logic Devices, Inc. & $57 \%$ & $70 \%$ & $38 \%$ & $48 \%$ \\
\hline 82 & LogicVision Inc & $-36 \%$ & $-61 \%$ & $-74 \%$ & \\
\hline 83 & LSI Corp & $-3 \%$ & $26 \%$ & $11 \%$ & $-31 \%$ \\
\hline 84 & Lumera Corp & $45 \%$ & $-12 \%$ & & \\
\hline
\end{tabular}




\begin{tabular}{|c|c|c|c|c|c|}
\hline 85 & Marvell Technology Group Ltd. & $-29 \%$ & $-6 \%$ & $59 \%$ & $147 \%$ \\
\hline 86 & MathStar Inc & $-48 \%$ & & & \\
\hline 87 & Maxim Integrated Products, Inc. & $-7 \%$ & $-22 \%$ & $-33 \%$ & $-27 \%$ \\
\hline 88 & Mellanox Technologies, Ltd. & & & & \\
\hline 89 & MEMC Elect. Materials, Inc. (US) & $68 \%$ & $275 \%$ & $462 \%$ & $1081 \%$ \\
\hline 90 & Metalink Ltd. & $24 \%$ & $35 \%$ & $-7 \%$ & $78 \%$ \\
\hline 91 & Micrel, Inc. & $-2 \%$ & $6 \%$ & $-17 \%$ & $-42 \%$ \\
\hline 92 & Microchip Technology, Inc. (US) & $9 \%$ & $32 \%$ & $28 \%$ & $56 \%$ \\
\hline 93 & Micromem Technologies, Inc. & $-32 \%$ & $-32 \%$ & $67 \%$ & $-34 \%$ \\
\hline 94 & Micron Technology Inc. & $-19 \%$ & $11 \%$ & $-12 \%$ & $-49 \%$ \\
\hline 95 & Micropac Industries, Inc. & $-24 \%$ & $8 \%$ & $117 \%$ & $283 \%$ \\
\hline 96 & Microsemi Corp. & $-16 \%$ & $17 \%$ & $72 \%$ & $169 \%$ \\
\hline 97 & Microtune Inc & $-6 \%$ & $-3 \%$ & $46 \%$ & $-60 \%$ \\
\hline 98 & Mindspeed Technologies Inc & $-23 \%$ & $-4 \%$ & $-64 \%$ & \\
\hline 99 & MIPS Technologies, Inc. (US) & $25 \%$ & $-2 \%$ & $47 \%$ & $30 \%$ \\
\hline 100 & Mirae Corp & $-14 \%$ & $-9 \%$ & $-29 \%$ & $-46 \%$ \\
\hline 101 & Monolithic Power Systems Inc & $8 \%$ & & & \\
\hline 102 & MoSys Inc & $13 \%$ & $51 \%$ & $4 \%$ & $-37 \%$ \\
\hline 103 & MRV Communications, Inc. & $20 \%$ & $17 \%$ & $4 \%$ & $30 \%$ \\
\hline 104 & National Semiconductor Corp. & $-1 \%$ & $26 \%$ & $32 \%$ & $98 \%$ \\
\hline 105 & NeoMagic Corp. & $-17 \%$ & $14 \%$ & $-74 \%$ & $-72 \%$ \\
\hline 106 & Netlist Inc & & & & \\
\hline 107 & Netlogic Microsystems Inc & $-12 \%$ & $91 \%$ & & \\
\hline 108 & Nova Measuring Instruments Ltd & $30 \%$ & $-10 \%$ & $-49 \%$ & $-3 \%$ \\
\hline 109 & NVE Corp & $75 \%$ & $51 \%$ & $-23 \%$ & $278 \%$ \\
\hline 110 & NVIDIA Corp & $62 \%$ & $204 \%$ & $281 \%$ & $107 \%$ \\
\hline 111 & OmniVision Technologies Inc & $-26 \%$ & $2 \%$ & $-28 \%$ & $249 \%$ \\
\hline 112 & ON Semiconductor Corp & $55 \%$ & $118 \%$ & $75 \%$ & $303 \%$ \\
\hline 113 & On Track Innovations (Israel) & $-46 \%$ & $-46 \%$ & $-31 \%$ & \\
\hline 114 & Opnext Inc & & & & \\
\hline 115 & OPTi, Inc. & $122 \%$ & $265 \%$ & $285 \%$ & $375 \%$ \\
\hline 116 & Optical Communications Prod. Inc. & $-30 \%$ & $-16 \%$ & $-43 \%$ & $-26 \%$ \\
\hline 117 & Optium Corp & & & & \\
\hline 118 & OSI Systems, Inc. & $24 \%$ & $39 \%$ & $25 \%$ & $23 \%$ \\
\hline 119 & Pericom Semiconductor Corp. & $20 \%$ & $3 \%$ & $-2 \%$ & $-15 \%$ \\
\hline 120 & Photronics, Inc. & $-10 \%$ & $-25 \%$ & $-17 \%$ & $-39 \%$ \\
\hline 121 & Pixelplus Co Ltd & & & & \\
\hline 122 & Pixelworks Inc & $-58 \%$ & $-81 \%$ & $-88 \%$ & $-84 \%$ \\
\hline 123 & PLX Technology Inc & $3 \%$ & $17 \%$ & $8 \%$ & $33 \%$ \\
\hline 124 & PMC-Sierra Inc. & $-16 \%$ & $-24 \%$ & $-53 \%$ & $-50 \%$ \\
\hline 125 & Power Integrations Inc. & $22 \%$ & $21 \%$ & $-6 \%$ & $38 \%$ \\
\hline 126 & QLogic Corp. & $-4 \%$ & $0 \%$ & $-7 \%$ & $-16 \%$ \\
\hline 127 & QuickLogic Corp & $-31 \%$ & $-13 \%$ & $-22 \%$ & $-25 \%$ \\
\hline 128 & Rambus Inc. (DE) & $-22 \%$ & $15 \%$ & $-21 \%$ & $180 \%$ \\
\hline 129 & Ramtron International Corp. (US) & $36 \%$ & $-2 \%$ & $-7 \%$ & $5 \%$ \\
\hline 130 & REMEC Inc & $93 \%$ & & & \\
\hline 131 & RF Micro Devices, Inc. (US) & $0 \%$ & $19 \%$ & $-19 \%$ & $-54 \%$ \\
\hline
\end{tabular}




\begin{tabular}{|c|c|c|c|c|c|}
\hline 132 & SatCon Technology Corp. & $-30 \%$ & $-32 \%$ & $-51 \%$ & $-63 \%$ \\
\hline 133 & Semiconductor Manufacturing Int. & $-7 \%$ & $-35 \%$ & & \\
\hline 134 & Semtech Corp. & $-8 \%$ & $-18 \%$ & $-32 \%$ & $-47 \%$ \\
\hline 135 & Sigmatel Inc & $-59 \%$ & $-87 \%$ & $-85 \%$ & \\
\hline 136 & Silicon Image Inc & $-13 \%$ & $-23 \%$ & $-11 \%$ & $58 \%$ \\
\hline 137 & Silicon Laboratories Inc & $-16 \%$ & $12 \%$ & $-26 \%$ & $25 \%$ \\
\hline 138 & Silicon Motion Technology Corp & $44 \%$ & & & \\
\hline 139 & Silicon Storage Technology, Inc. & $-7 \%$ & $-14 \%$ & $-61 \%$ & $-51 \%$ \\
\hline 140 & Simtek Corp. & $71 \%$ & $-1 \%$ & $-53 \%$ & $57 \%$ \\
\hline 141 & SIPEX Corp & & & & \\
\hline 142 & Sirenza Microdevices Inc & $26 \%$ & $160 \%$ & $119 \%$ & $158 \%$ \\
\hline 143 & SiRf Technology Holdings Inc & $-17 \%$ & $55 \%$ & & \\
\hline 144 & Skyworks Solutions, Inc. (US) & $31 \%$ & $-5 \%$ & $-23 \%$ & $-46 \%$ \\
\hline 145 & Solarfun Power Holdings Co Ltd & & & & \\
\hline 146 & Solitron Devices, Inc. (US) & $-31 \%$ & $94 \%$ & $152 \%$ & $479 \%$ \\
\hline 147 & Spansion Inc & & & & \\
\hline 148 & Spatializer Audio Laboratories, Inc. & & & & \\
\hline 149 & SRS Labs, Inc. & $83 \%$ & $88 \%$ & $55 \%$ & $275 \%$ \\
\hline 150 & Staktek Holdings Inc & $-32 \%$ & $9 \%$ & & \\
\hline 151 & Standard Microsystems Corp. & $16 \%$ & $53 \%$ & $30 \%$ & $72 \%$ \\
\hline 152 & STATS ChipPac Ltd & $46 \%$ & $54 \%$ & $1 \%$ & $-14 \%$ \\
\hline 153 & SunPower Corp & & & & \\
\hline 154 & Suntech Power Holdings Co Ltd & & & & \\
\hline 155 & Supertex, Inc. & $0 \%$ & $75 \%$ & $114 \%$ & $123 \%$ \\
\hline 156 & Taiwan Semiconductor Mfg. & $18 \%$ & $43 \%$ & $47 \%$ & $18 \%$ \\
\hline 157 & Techwell Inc & $13 \%$ & & & \\
\hline 158 & Tessera Technologies Inc & $30 \%$ & $15 \%$ & & \\
\hline 159 & Texas Instruments Inc. & $5 \%$ & $21 \%$ & $26 \%$ & $25 \%$ \\
\hline 160 & Toshiba Ceramics Co., Ltd. (Jp) & & & & \\
\hline 161 & Tower Semiconductor Ltd. & $18 \%$ & $2 \%$ & $-71 \%$ & $-70 \%$ \\
\hline 162 & Transmeta Corp. (Del) & $-51 \%$ & $-42 \%$ & $-74 \%$ & $-68 \%$ \\
\hline 163 & TranSwitch Corp. & $-17 \%$ & $1 \%$ & $-28 \%$ & $-33 \%$ \\
\hline 164 & Trident Microsystems, Inc. & $-13 \%$ & $75 \%$ & $158 \%$ & $853 \%$ \\
\hline 165 & Trio-Tech International & $128 \%$ & $308 \%$ & $301 \%$ & $514 \%$ \\
\hline 166 & Tripath Technology Inc & & & & \\
\hline 167 & TriQuint Semiconductor, Inc. & $0 \%$ & $33 \%$ & $-22 \%$ & $-51 \%$ \\
\hline 168 & Ultra Clean Holdings Inc & $71 \%$ & $133 \%$ & & \\
\hline 169 & United Microelectronics C. (China) & $8 \%$ & $-4 \%$ & $-22 \%$ & $-47 \%$ \\
\hline 170 & Varian Semiconductor Eq. Ass. & $82 \%$ & $122 \%$ & $120 \%$ & $158 \%$ \\
\hline 171 & Verigy Ltd & & & & \\
\hline 172 & Vimicro International Corp & & & & \\
\hline 173 & Virage Logic Corp & $-21 \%$ & $-34 \%$ & $-19 \%$ & $-44 \%$ \\
\hline 174 & Volterra Semiconductor Corp & $-10 \%$ & $-6 \%$ & & \\
\hline 175 & White Electronic Designs Corp. & $9 \%$ & $6 \%$ & $-20 \%$ & $-17 \%$ \\
\hline 176 & WJ Communications Inc & $-7 \%$ & $-24 \%$ & $-57 \%$ & $-28 \%$ \\
\hline 177 & Xilinx, Inc. & $7 \%$ & $-7 \%$ & $-22 \%$ & $-3 \%$ \\
\hline 178 & Yingli Green Energy Holding Co. & & & & \\
\hline
\end{tabular}




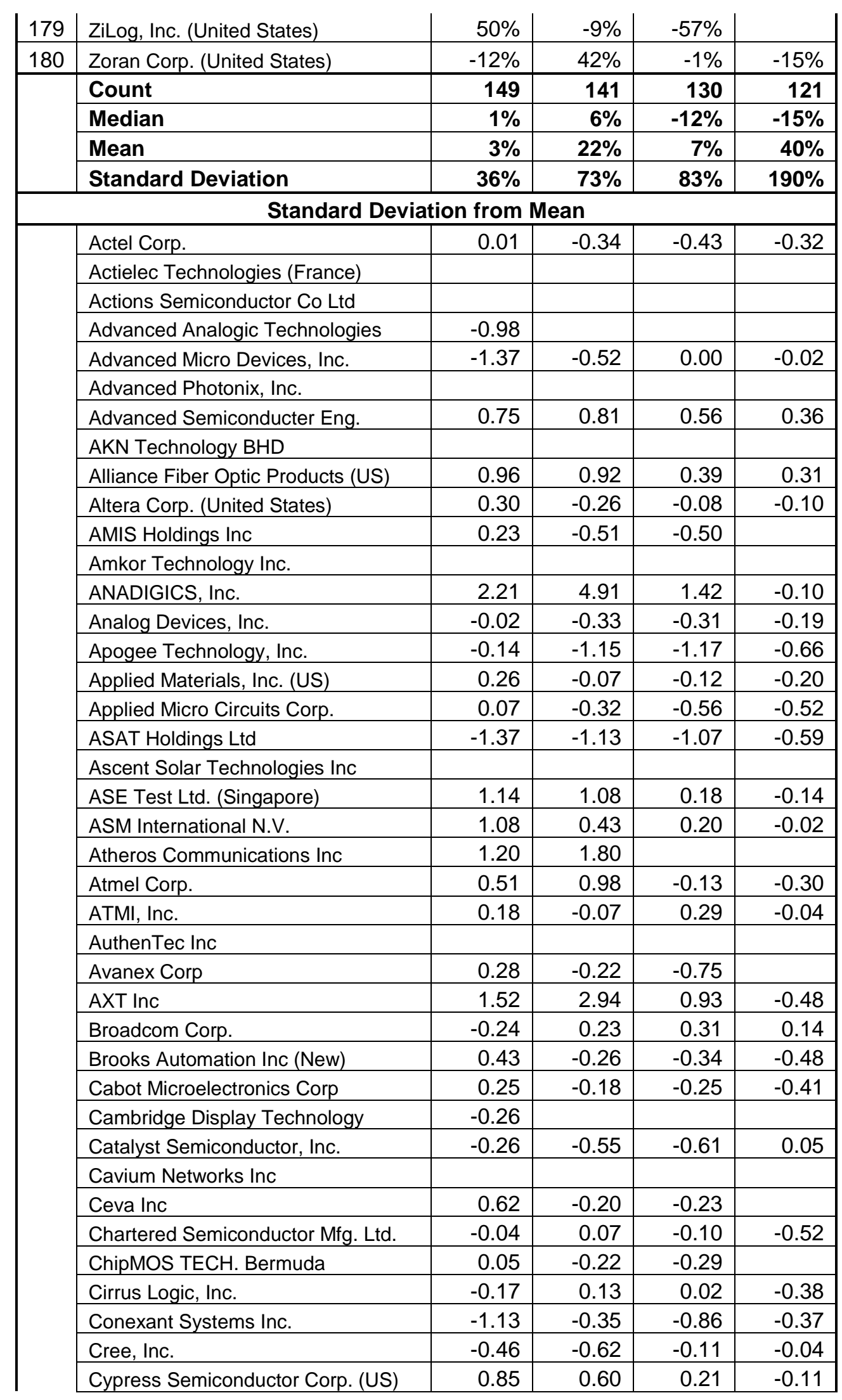




\begin{tabular}{|c|c|c|c|c|}
\hline DayStar Technologies Inc & -1.60 & -0.78 & & \\
\hline Diodes, Inc. & 0.22 & 1.10 & 2.08 & 5.47 \\
\hline DPAC Technologies Corp. & -0.25 & -1.35 & -1.18 & -0.72 \\
\hline DSP Group, Inc. (United States) & -0.73 & -0.54 & -0.30 & -0.15 \\
\hline eMagin Corp (DE) & -2.21 & -1.51 & -1.21 & -0.67 \\
\hline EMCORE Corp. (United States) & -0.77 & 0.56 & 0.55 & -0.34 \\
\hline Epcos AG & 1.04 & 0.26 & -0.17 & -0.45 \\
\hline ESS Technology, Inc. & -1.55 & -1.33 & -1.17 & -0.70 \\
\hline Evergreen Solar Inc. & -0.79 & 0.44 & 2.90 & 1.31 \\
\hline Exar Corp. & -0.05 & -0.40 & -0.31 & -0.37 \\
\hline Fairchild Semiconductor Int. & -0.10 & -0.08 & -0.24 & -0.32 \\
\hline Finisar Corp (United States) & 0.16 & 1.71 & 0.47 & -0.42 \\
\hline \multicolumn{5}{|l|}{ First Solar Inc } \\
\hline Focus Enhancements, Inc. & 1.13 & 0.10 & -0.47 & -0.26 \\
\hline Formfactor Inc & 0.23 & 0.61 & 1.11 & \\
\hline Genesis Microchip Inc (DE) & -1.35 & -0.98 & -0.61 & -0.58 \\
\hline \multicolumn{5}{|l|}{ GSI Technology Inc } \\
\hline HEI Inc & -1.66 & -1.08 & -0.75 & -0.64 \\
\hline $\mathrm{Hi} /$ fn Inc. & 0.06 & -0.46 & -0.58 & -0.41 \\
\hline \multicolumn{5}{|l|}{ Himax Technologies Inc } \\
\hline Hittite Microwave Corp & 0.44 & & & \\
\hline \multicolumn{5}{|l|}{ Hologram Ind.Marne La Vallee (Fr.) } \\
\hline Ibis Technology Corp. & -1.43 & -0.75 & -1.10 & -0.65 \\
\hline Integrated Device Technology, Inc. & 0.18 & 0.19 & 0.00 & -0.39 \\
\hline Integrated Silicon Solution, Inc. & -0.20 & -0.58 & -0.77 & -0.42 \\
\hline Intel Corp & 0.14 & -0.38 & -0.29 & -0.26 \\
\hline International Rectifier Corp. & -0.06 & -0.52 & -0.26 & -0.16 \\
\hline Intersil Corp. & 0.17 & 0.51 & 0.29 & -0.14 \\
\hline \multicolumn{5}{|l|}{ IPG Photonics Corp } \\
\hline Irvine Sensors Corp. & -1.02 & -0.79 & -0.61 & -0.21 \\
\hline Isonics Corp. & -2.05 & -1.51 & -0.90 & -0.53 \\
\hline IXYS Corp. & -0.20 & -0.45 & 0.07 & -0.05 \\
\hline \multicolumn{5}{|l|}{ JA Solar Holdings Co Ltd } \\
\hline \multicolumn{5}{|l|}{ Jazz Technologies Inc } \\
\hline JDS Uniphase Corp & -0.92 & -0.41 & -0.67 & -0.54 \\
\hline Kulicke \& Soffa Industries, Inc. & 0.08 & 0.06 & -0.31 & -0.38 \\
\hline Lattice Semiconductor Corp. & -0.07 & -0.07 & -0.41 & -0.52 \\
\hline Leadis Technology Inc & -0.74 & -0.95 & & \\
\hline LightPath Technologies, Inc. & 0.65 & 0.41 & 0.01 & -0.56 \\
\hline Linear Technology Corp. (US) & -0.11 & -0.42 & -0.22 & -0.21 \\
\hline Logic Devices, Inc. & 1.50 & 0.66 & 0.37 & 0.04 \\
\hline LogicVision Inc & -1.09 & -1.14 & -0.97 & \\
\hline LSI Corp & -0.17 & 0.05 & 0.05 & -0.37 \\
\hline Lumera Corp & 1.18 & -0.47 & & \\
\hline Marvell Technology Group Ltd. & -0.89 & -0.38 & 0.62 & 0.56 \\
\hline MathStar Inc & -1.42 & & & \\
\hline Maxim Integrated Products, Inc. & -0.28 & -0.61 & -0.49 & -0.35 \\
\hline
\end{tabular}




\begin{tabular}{|c|c|c|c|c|}
\hline \multicolumn{5}{|l|}{ Mellanox Technologies, Ltd. } \\
\hline MEMC Elect. Materials, Inc. (US) & 1.81 & 3.47 & 5.46 & 5.49 \\
\hline Metalink Ltd. & 0.59 & 0.18 & -0.17 & 0.20 \\
\hline Micrel, Inc. & -0.13 & -0.22 & -0.29 & -0.43 \\
\hline Microchip Technology, Inc. (US) & 0.18 & 0.14 & 0.25 & 0.08 \\
\hline Micromem Technologies, Inc. & -0.98 & -0.75 & 0.72 & -0.39 \\
\hline Micron Technology Inc. & -0.60 & -0.16 & -0.23 & -0.47 \\
\hline Micropac Industries, Inc. & -0.77 & -0.20 & 1.32 & 1.28 \\
\hline Microsemi Corp. & -0.54 & -0.07 & 0.78 & 0.68 \\
\hline Microtune Inc & -0.24 & -0.34 & 0.47 & -0.53 \\
\hline Mindspeed Technologies Inc & -0.73 & -0.36 & -0.86 & \\
\hline MIPS Technologies, Inc. (US) & 0.61 & -0.33 & 0.47 & -0.05 \\
\hline Mirae Corp & -0.48 & -0.43 & -0.44 & -0.45 \\
\hline Monolithic Power Systems Inc & 0.14 & & & \\
\hline MoSys Inc & 0.29 & 0.40 & -0.04 & -0.41 \\
\hline MRV Communications, Inc. & 0.49 & -0.07 & -0.04 & -0.05 \\
\hline National Semiconductor Corp. & -0.11 & 0.05 & 0.30 & 0.30 \\
\hline NeoMagic Corp. & -0.55 & -0.11 & -0.98 & -0.59 \\
\hline \multicolumn{5}{|l|}{ Netlist Inc } \\
\hline Netlogic Microsystems Inc & -0.42 & 0.94 & & \\
\hline Nova Measuring Instruments Ltd & 0.77 & -0.44 & -0.67 & -0.23 \\
\hline NVE Corp & 2.02 & 0.40 & -0.36 & 1.25 \\
\hline NVIDIA Corp & 1.65 & 2.49 & 3.29 & 0.35 \\
\hline OmniVision Technologies Inc & -0.81 & -0.27 & -0.42 & 1.10 \\
\hline ON Semiconductor Corp & 1.46 & 1.31 & 0.81 & 1.39 \\
\hline On Track Innovations (Israel) & -1.37 & -0.93 & -0.47 & \\
\hline \multicolumn{5}{|l|}{ Opnext Inc } \\
\hline OPTi, Inc. & 3.34 & 3.33 & 3.33 & 1.76 \\
\hline Optical Communications Prod. Inc. & -0.91 & -0.52 & -0.61 & -0.35 \\
\hline \multicolumn{5}{|l|}{ Optium Corp } \\
\hline OSI Systems, Inc. & 0.60 & 0.22 & 0.21 & -0.09 \\
\hline Pericom Semiconductor Corp. & 0.49 & -0.27 & -0.11 & -0.29 \\
\hline Photronics, Inc. & -0.36 & -0.64 & -0.29 & -0.42 \\
\hline \multicolumn{5}{|l|}{ Pixelplus Co Ltd } \\
\hline Pixelworks Inc & -1.70 & -1.42 & -1.14 & -0.65 \\
\hline PLX Technology Inc & 0.02 & -0.07 & 0.01 & -0.04 \\
\hline PMC-Sierra Inc. & -0.52 & -0.63 & -0.73 & -0.47 \\
\hline Power Integrations Inc. & 0.54 & -0.02 & -0.16 & -0.01 \\
\hline QLogic Corp. & -0.21 & -0.31 & -0.17 & -0.30 \\
\hline QuickLogic Corp & -0.96 & -0.49 & -0.35 & -0.34 \\
\hline Rambus Inc. (DE) & -0.69 & -0.10 & -0.33 & 0.74 \\
\hline Ramtron International Corp. (US) & 0.92 & -0.33 & -0.17 & -0.18 \\
\hline REMEC Inc & 2.51 & & & \\
\hline RF Micro Devices, Inc. (US) & -0.08 & -0.04 & -0.31 & -0.49 \\
\hline SatCon Technology Corp. & -0.93 & -0.74 & -0.70 & -0.54 \\
\hline Semiconductor Manufacturing Int. & -0.27 & -0.79 & & \\
\hline Semtech Corp. & -0.30 & -0.55 & -0.47 & -0.46 \\
\hline
\end{tabular}




\begin{tabular}{|c|c|c|c|c|}
\hline Sigmatel Inc & -1.72 & -1.50 & -1.11 & \\
\hline Silicon Image Inc & -0.44 & -0.62 & -0.22 & 0.09 \\
\hline Silicon Laboratories Inc & -0.52 & -0.14 & -0.41 & -0.08 \\
\hline Silicon Motion Technology Corp & 1.16 & & & \\
\hline Silicon Storage Technology, Inc. & -0.27 & -0.49 & -0.82 & -0.48 \\
\hline Simtek Corp. & 1.91 & -0.32 & -0.73 & 0.09 \\
\hline \multicolumn{5}{|l|}{ SIPEX Corp } \\
\hline Sirenza Microdevices Inc & 0.65 & 1.88 & 1.34 & 0.62 \\
\hline SiRf Technology Holdings Inc & -0.55 & 0.45 & & \\
\hline Skyworks Solutions, Inc. (US) & 0.79 & -0.37 & -0.36 & -0.46 \\
\hline \multicolumn{5}{|l|}{ Solarfun Power Holdings Co Ltd } \\
\hline Solitron Devices, Inc. (US) & -0.96 & 0.98 & 1.74 & 2.31 \\
\hline \multicolumn{5}{|l|}{ Spansion Inc } \\
\hline \multicolumn{5}{|l|}{ Spatializer Audio Laboratories, Inc. } \\
\hline SRS Labs, Inc. & 2.23 & 0.90 & 0.57 & 1.24 \\
\hline Staktek Holdings Inc & -0.97 & -0.18 & & \\
\hline Standard Microsystems Corp. & 0.35 & 0.42 & 0.27 & 0.17 \\
\hline STATS ChipPac Ltd & 1.22 & 0.44 & -0.07 & -0.29 \\
\hline \multicolumn{5}{|l|}{ SunPower Corp } \\
\hline \multicolumn{5}{|l|}{ Suntech Power Holdings Co Ltd } \\
\hline Supertex, Inc. & -0.09 & 0.73 & 1.28 & 0.44 \\
\hline Taiwan Semiconductor Mfg. & 0.42 & 0.29 & 0.47 & -0.11 \\
\hline Techwell Inc & 0.29 & & & \\
\hline Tessera Technologies Inc & 0.77 & -0.09 & & \\
\hline Texas Instruments Inc. & 0.07 & -0.01 & 0.22 & -0.08 \\
\hline \multicolumn{5}{|l|}{ Toshiba Ceramics Co., Ltd. (Jp) } \\
\hline Tower Semiconductor Ltd. & 0.42 & -0.28 & -0.94 & -0.58 \\
\hline Transmeta Corp. (Del) & -1.51 & -0.87 & -0.98 & -0.57 \\
\hline TranSwitch Corp. & -0.54 & -0.29 & -0.43 & -0.38 \\
\hline Trident Microsystems, Inc. & -0.46 & 0.73 & 1.81 & 4.29 \\
\hline Trio-Tech International & 3.50 & 3.92 & 3.54 & 2.50 \\
\hline \multicolumn{5}{|l|}{ Tripath Technology Inc } \\
\hline TriQuint Semiconductor, Inc. & -0.07 & 0.15 & -0.36 & -0.48 \\
\hline Ultra Clean Holdings Inc & 1.90 & 1.52 & & \\
\hline United Microelectronics C. (China) & 0.13 & -0.36 & -0.35 & -0.46 \\
\hline Varian Semiconductor Eq. Ass. & 2.22 & 1.37 & 1.36 & 0.62 \\
\hline \multicolumn{5}{|l|}{ Verigy Ltd } \\
\hline \multicolumn{5}{|l|}{ Vimicro International Corp } \\
\hline Virage Logic Corp & -0.66 & -0.77 & -0.31 & -0.44 \\
\hline Volterra Semiconductor Corp & -0.35 & -0.39 & & \\
\hline White Electronic Designs Corp. & 0.17 & -0.22 & -0.33 & -0.30 \\
\hline WJ Communications Inc & -0.27 & -0.63 & -0.77 & -0.36 \\
\hline Xilinx, Inc. & 0.11 & -0.40 & -0.36 & -0.23 \\
\hline \multicolumn{5}{|l|}{ Yingli Green Energy Holding Co. } \\
\hline ZiLog, Inc. (United States) & 1.31 & -0.42 & -0.77 & \\
\hline Zoran Corp. (United States) & -0.42 & 0.28 & -0.10 & -0.29 \\
\hline
\end{tabular}




\section{Appendix H: NAICS Codes and Companies per Code}

NACS Codes:

Futlicly Traded Companice - Mergent Online (11/4,0e - 11/11,08)

USExchange Dnty - United States - Any Index

\begin{tabular}{|c|c|c|c|c|c|}
\hline 2 Digit Category & $\begin{array}{l}\text { 2-Digit Code } \\
\text { मे Companies }\end{array}$ & \begin{tabular}{|l|} 
4Digit \\
Code
\end{tabular} & $\mid \begin{array}{l}\text { 4Digit Code } \\
\text { \# Companies }\end{array}$ & $\left|\begin{array}{l}\text { 3-Digit Code } \\
\text { \# Companies }\end{array}\right|$ & 3-Digit C ategory \\
\hline \multirow[t]{5}{*}{ Agriculture, Forestry } & 46 & $\begin{array}{l}1111 \\
1112 \\
1113 \\
1114 \\
1119\end{array}$ & $\begin{array}{l}6 \\
1 \\
4 \\
3 \\
3\end{array}$ & 17 & Crop Production (111) \\
\hline & & $\begin{array}{l}1121 \\
1122 \\
1123 \\
1124 \\
1125 \\
1129\end{array}$ & $\begin{array}{l}1 \\
1 \\
2 \\
2 \\
3 \\
\end{array}$ & 9 & Animal Froduction (112) \\
\hline & & $\begin{array}{l}1131 \\
1132 \\
1130\end{array}$ & $\begin{array}{l}4 \\
2\end{array}$ & 6 & Forestry and Logging (113) \\
\hline & & $\begin{array}{l}1141 \\
1142\end{array}$ & 2 & 2 & Fishing. Hunting and Trapping (114) \\
\hline & & $\begin{array}{l}1151 \\
1152 \\
1153\end{array}$ & $\begin{array}{l}7 \\
2 \\
3\end{array}$ & 12 & Support Actuities for Agrieulture $\&$ Forestry $(115)$ \\
\hline \multirow[t]{3}{*}{ Wining (21) } & 655 & 2111 & 292 & 292 & Qil and 6 as Extraction (211) \\
\hline & & $\begin{array}{l}2121 \\
2122 \\
2123\end{array}$ & $\begin{array}{r}23 \\
15 \\
28\end{array}$ & 208 & Mining (exoept Dil and oas - 212) \\
\hline & & 2131 & 156 & 155 & Support Acturities for Mining (213) \\
\hline Utilitie (22) & 274 & $\begin{array}{l}2211 \\
2212 \\
2213\end{array}$ & $\begin{array}{r}174 \\
69 \\
31\end{array}$ & 274 & Utilities (221) \\
\hline \multirow[t]{3}{*}{ Constrution (23) } & 133 & $\begin{array}{l}2601 \\
2602\end{array}$ & $\begin{array}{l}43 \\
12 \\
\end{array}$ & 55 & Construction of Euilding (236) \\
\hline & & $\begin{array}{l}2371 \\
2372 \\
2373 \\
2379\end{array}$ & $\begin{array}{r}29 \\
25 \\
4 \\
1\end{array}$ & 59 & Heawy and Ciwil Enginesring Construction (237) \\
\hline & & $\begin{array}{l}2301 \\
2302 \\
238 \\
230\end{array}$ & $\begin{array}{r}3 \\
13 \\
3\end{array}$ & 19 & Specialty Trade Contractors (238) \\
\hline \multirow[t]{3}{*}{ Manufacturing 01} & 336 & $\begin{array}{l}3111 \\
3112 \\
3113 \\
3114 \\
3116 \\
3116 \\
3117 \\
3118 \\
3119\end{array}$ & $\begin{array}{r}3 \\
16 \\
11 \\
20 \\
15 \\
25 \\
1 \\
17 \\
34\end{array}$ & 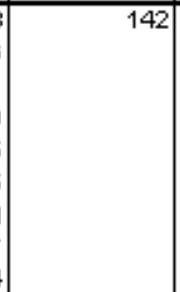 & Food Manufacturing (911) \\
\hline & & $\begin{array}{l}3121 \\
3122\end{array}$ & $\begin{array}{l}49 \\
13\end{array}$ & 62 & Eewerage 3 Tobaco Froduct Manufacturing (312) \\
\hline & & $\begin{array}{l}3131 \\
3132 \\
3130\end{array}$ & $\begin{array}{r}1 \\
17 \\
3 \\
\end{array}$ & 21 & Textile Mille (313) \\
\hline
\end{tabular}


NACS Codes:

Publioly Traded Companies - Mergent Dnline (11/4ns - 11/11,08)

US Exohange Drity - United States - Any Index

\begin{tabular}{|c|c|c|c|c|c|}
\hline 2-Digit Category & $\begin{array}{l}\text { 2- Digit Code } \\
\text { स्म Companies }\end{array}$ & \begin{tabular}{|l}
40 igit \\
Code
\end{tabular} & $\begin{array}{l}\text { 4Digit Coude } \\
\text { Hompanies }\end{array}$ & $\begin{array}{l}\text { 3- Digit Code } \\
\text { Hompanies }\end{array}$ & 3-D igit C: ategory \\
\hline & & $\begin{array}{l}3141 \\
3149\end{array}$ & $\begin{array}{l}8 \\
4\end{array}$ & 12 & Textile Froduot hulile $(314)$ \\
\hline & & $\begin{array}{l}3151 \\
3152 \\
3159\end{array}$ & $\begin{array}{r}2 \\
51 \\
13 \\
\end{array}$ & $\bar{B} \bar{B}$ & A.pparel bulanfacturing $(315)$ \\
\hline & & $\begin{array}{l}3101 \\
3162 \\
3169\end{array}$ & $\begin{array}{r}28 \\
5\end{array}$ & 33 & Leather and Allied Froduct Manufacturing (316) \\
\hline \multirow[t]{7}{*}{ Wul anutacturing 32$)$} & \multirow[t]{7}{*}{1,159} & $\begin{array}{l}3211 \\
3212 \\
3219\end{array}$ & $\begin{array}{r}4 \\
5 \\
20\end{array}$ & 29 & "úlood Product banufacturing (321) \\
\hline & & $\begin{array}{l}3221 \\
3222\end{array}$ & 38 & 71 & Paper hanufacturing (K2) \\
\hline & & 3231 & 4 & 42 & Frinting and Fielated Support Actrities (923) \\
\hline & & 3241 & 52 & 52 & Fetroleum and Coal Froduct Manufacturing (324) \\
\hline & & $\begin{array}{l}3251 \\
3252 \\
3253 \\
3254 \\
3255 \\
3256 \\
3259\end{array}$ & $\begin{array}{r}56 \\
20 \\
29 \\
631 \\
19 \\
50 \\
31 \\
\end{array}$ & 844 & Chemioal hanufacturing (326) \\
\hline & & $\begin{array}{l}3201 \\
3202 \\
\end{array}$ & 60 & 73 & $\begin{array}{l}\text { Plastics and Fiubber Froduct } \\
\text { blanutacturing (320) }\end{array}$ \\
\hline & & $\begin{array}{l}3271 \\
3272 \\
3273 \\
3274 \\
3279\end{array}$ & $\begin{array}{r}9 \\
10 \\
15 \\
2 \\
12\end{array}$ & 48 & $\begin{array}{l}\text { Nonmetallic hineral Froduct } \\
\text { Manufacturing ( } 327)\end{array}$ \\
\hline \multirow[t]{4}{*}{ Wanufacturing (33) } & \multirow[t]{4}{*}{2,474} & $\begin{array}{l}3311 \\
3312 \\
3313 \\
3314 \\
3315\end{array}$ & $\begin{array}{l}31 \\
14 \\
12 \\
25 \\
16\end{array}$ & 98 & Primary Malal Manufacturing (331) \\
\hline & & $\begin{array}{l}3321 \\
3322 \\
333 \\
3324 \\
3325 \\
3326 \\
3327 \\
3328 \\
3329\end{array}$ & $\begin{array}{r}12 \\
8 \\
28 \\
11 \\
7 \\
2 \\
8 \\
9 \\
40\end{array}$ & 120 & $\begin{array}{l}\text { Fabricated lotal Froduct } \\
\text { Manufacturing ( } 3: 2)\end{array}$ \\
\hline & & $\begin{array}{l}3301 \\
3532 \\
3333 \\
3334 \\
3535 \\
3330 \\
3339\end{array}$ & $\begin{array}{l}59 \\
59 \\
86 \\
34 \\
18 \\
22 \\
52\end{array}$ & 329 & Wachinery Manufacturing (oso) \\
\hline & & 3341 & 213 & 1,244 & Computer and Eleotronio: Froduot \\
\hline
\end{tabular}


NAL: S Codes:

Fublicly Traded Domparie - Wergent Dnline (1 1,4/08 - 1 1/1108)

US Exohange Dnty - United States - Any Index

Total 19.720 6-Digit Codes

\begin{tabular}{|c|c|c|c|c|c|}
\hline 2- Digit Dategory & $\begin{array}{l}\text { 2-Digit Code } \\
\text { H Companies }\end{array}$ & \begin{tabular}{l|}
$40 \mathrm{igit}$ \\
Code
\end{tabular} & $\begin{array}{l}\text { 4Digit Code } \\
\text { Lompanies }\end{array}$ & $\begin{array}{l}\text { 3- Digit Code } \\
\text { Dompanies }\end{array}$ & 3-D igit c: ategory \\
\hline & & $\begin{array}{l}3342 \\
3343 \\
3344 \\
3345 \\
3346\end{array}$ & $\begin{array}{r}274 \\
17 \\
331 \\
302 \\
40\end{array}$ & & Mianufacturing (394) \\
\hline & & $\begin{array}{l}3351 \\
3352 \\
3353 \\
3359\end{array}$ & $\begin{array}{l}25 \\
12 \\
28 \\
68\end{array}$ & 133 & $\begin{array}{l}\text { Electrical Equipment, Applianoe, and Component } \\
\text { Wanutacturing (3\%) }\end{array}$ \\
\hline & & $\begin{array}{l}3301 \\
3362 \\
3363 \\
3364 \\
3365 \\
3360 \\
3369\end{array}$ & $\begin{array}{r}5 \\
22 \\
60 \\
50 \\
5 \\
10 \\
14\end{array}$ & 176 & Transportation Equipment Manufacturing (936) \\
\hline & & $\begin{array}{l}3371 \\
3372 \\
3379\end{array}$ & $\begin{array}{r}22 \\
11 \\
2\end{array}$ & 35 & $\begin{array}{l}\text { Furniture and Fielated Froduct } \\
\text { Wanufacturing (SBT) }\end{array}$ \\
\hline & & 3391 & $\begin{array}{r}24 \\
92\end{array}$ & 339 & Wizcellaneous hanufacturing (399) \\
\hline \multirow[t]{3}{*}{ 'in' holes ale (42) } & 429 & $\begin{array}{l}4231 \\
4232 \\
4293 \\
4294 \\
4295 \\
4230 \\
4237 \\
4239 \\
4239\end{array}$ & $\begin{array}{r}24 \\
4 \\
16 \\
102 \\
15 \\
55 \\
7 \\
37 \\
28\end{array}$ & 288 & Werohant bónolesalers, Durable $600 \mathrm{ds}(423)$ \\
\hline & & $\begin{array}{l}4241 \\
4242 \\
4243 \\
4244 \\
4245 \\
4246 \\
4247 \\
4248 \\
4249\end{array}$ & $\begin{array}{r}10 \\
27 \\
7 \\
31 \\
6 \\
22 \\
13 \\
2 \\
20\end{array}$ & 138 & Wer ohant 'ónholesalers, Nondurable $600 d s(424)$ \\
\hline & & 4251 & 2 & 2 & $\begin{array}{l}\text { 'ol'tholesale Electonic halkets and Agents and } \\
\text { Erokers (425) }\end{array}$ \\
\hline \multirow[t]{4}{*}{ Fi etail Trade (44) } & 305 & $\begin{array}{l}4411 \\
4412 \\
4413\end{array}$ & $\begin{array}{r}9 \\
20 \\
14\end{array}$ & 43 & Wotor Wehiole and Farts Dealers (441) \\
\hline & & $\begin{array}{l}4421 \\
4422 \\
\end{array}$ & $\begin{array}{r}12 \\
8 \\
\end{array}$ & 20 & Furniture and Home F urizhings Stores (442) \\
\hline & & $\begin{array}{l}441 \\
4441 \\
4442\end{array}$ & $\begin{array}{r}31 \\
13 \\
4\end{array}$ & 48 & Electronis and Appliance Stores (443) \\
\hline & & $\begin{array}{l}4451 \\
4452\end{array}$ & 37 & 43 & Food and Beverage Stores (445) \\
\hline
\end{tabular}


NACS Codes:

Fublicly Traded Companies - Mergent Dnline (11/4,08-11/11,08)

USExohange Dnhy - United States - Any Index

Total 19.720 6-Digit Code

\begin{tabular}{|c|c|c|c|c|c|}
\hline \multirow[t]{7}{*}{ 2-Digit Category } & $\begin{array}{l}\text { 2- Digit Code } \\
\text { \# Companies }\end{array}$ & \begin{tabular}{|l|} 
4Digit \\
Code
\end{tabular} & $\begin{array}{l}\text { 4Digit Code } \\
\text { \#̈ Companies }\end{array}$ & \begin{tabular}{|l|} 
3- Digit Code \\
\# Companies
\end{tabular} & 3-Digit C ategany \\
\hline & & 4453 & & & \\
\hline & & 4451 & 5 & 57 & \multirow{2}{*}{$\begin{array}{l}\text { Heath and Personal Care Stores (446) } \\
\text { Gasoline Stations (447) }\end{array}$} \\
\hline & & 441 & 7 & 7 & \\
\hline & & 4491 & 68 & 87 & Clothing and Clothing Aceses ories Stores (449) \\
\hline & & 4492 & 9 & & \\
\hline & & 4483 & 12 & & \\
\hline \multirow[t]{11}{*}{ F etail Trade (46) } & \multirow[t]{11}{*}{203} & 4511 & 29 & 44 & Sporting Goods, Hobby, Eook, Musio Stores (451) \\
\hline & & 4512 & 15 & & \\
\hline & & 4021 & 20 & 49 & General Werchandie Stores (452) \\
\hline & & 4029 & 29 & & \\
\hline & & 4531 & 4 & 39 & Misceellaneous Store Rietailers (468) \\
\hline & & 4532 & 9 & & \\
\hline & & 4633 & & & \\
\hline & & 4609 & 26 & & \\
\hline & & 4541 & 61 & 71 & Nonstore Rietailers (454) \\
\hline & & 4642 & 1 & & \\
\hline & & 4643 & $g$ & & \\
\hline \multirow[t]{24}{*}{ Transportation and ' } & \multirow[t]{24}{*}{239} & 4811 & 29 & 38 & \multirow[t]{2}{*}{ Air Transportation (481) } \\
\hline & & 4812 & 9 & & \\
\hline & & 4821 & 18 & 18 & Riail Trare portation(182) \\
\hline & & 4831 & 15 & 20 & 'Iili' ater Transportation (483) \\
\hline & & 4852 & 5 & & 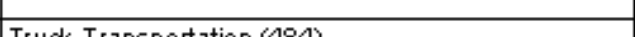 \\
\hline & & 4002 & 45 & 5.3 & \\
\hline & & 4851 & & 4 & \begin{tabular}{|l} 
Transit and Ground Fassenger Transportation (485) \\
\end{tabular} \\
\hline & & 4052 & & & \\
\hline & & 4053 & 1 & & \\
\hline & & 4054 & 1 & & \\
\hline & & 485 & 1 & & \\
\hline & & 4959 & 1 & & \\
\hline & & 4081 & 5 & 62 & \multirow[t]{3}{*}{ Fipeline Trans portation(486) } \\
\hline & & 4062 & 46 & & \\
\hline & & 4009 & 12 & & \\
\hline & & 4071 & & 1 & \multirow[t]{3}{*}{ Scenic and Sights eaing Transportation (497) } \\
\hline & & 4972 & 1 & & \\
\hline & & 4979 & & & \\
\hline & & 4881 & 6 & 49 & \multirow[t]{6}{*}{ S Support Actinities for Transportation (488) } \\
\hline & & 4882 & 2 & & \\
\hline & & 4883 & 8 & & \\
\hline & & 4884 & 2 & & \\
\hline & & 4885 & 16 & & \\
\hline & & 4989 & 9 & & \\
\hline Transportation and ' & 17 & 4911 & & & \\
\hline & & 4921 & 7 & 7 & Couriers and Mesengers (492) \\
\hline & & 4922 & & & \\
\hline & & 4901 & 10 & 10 & 'ulit arehousing and Storage (490) \\
\hline Information (51) & 1,003 & 5111 & $\pi$ & 502 & Fublikhing Indut tries (except Internet - 511) \\
\hline & & 5112 & 425 & & \\
\hline & & 5121 & 67 & 72 & Motion Ficture and Sound Riecording Indes tries (512) \\
\hline & & 5122 & 5 & & \\
\hline & & 5151 & 4 & 60 & Eroadcasting (except Internet - 515) \\
\hline
\end{tabular}


NAICS Codes:

Publisly Traded Companies - Mergent Dnine (11/4ng - 11/11,08)

US Exchange Dnty - United States - Any Index

Total 19.720 B-Digit Code

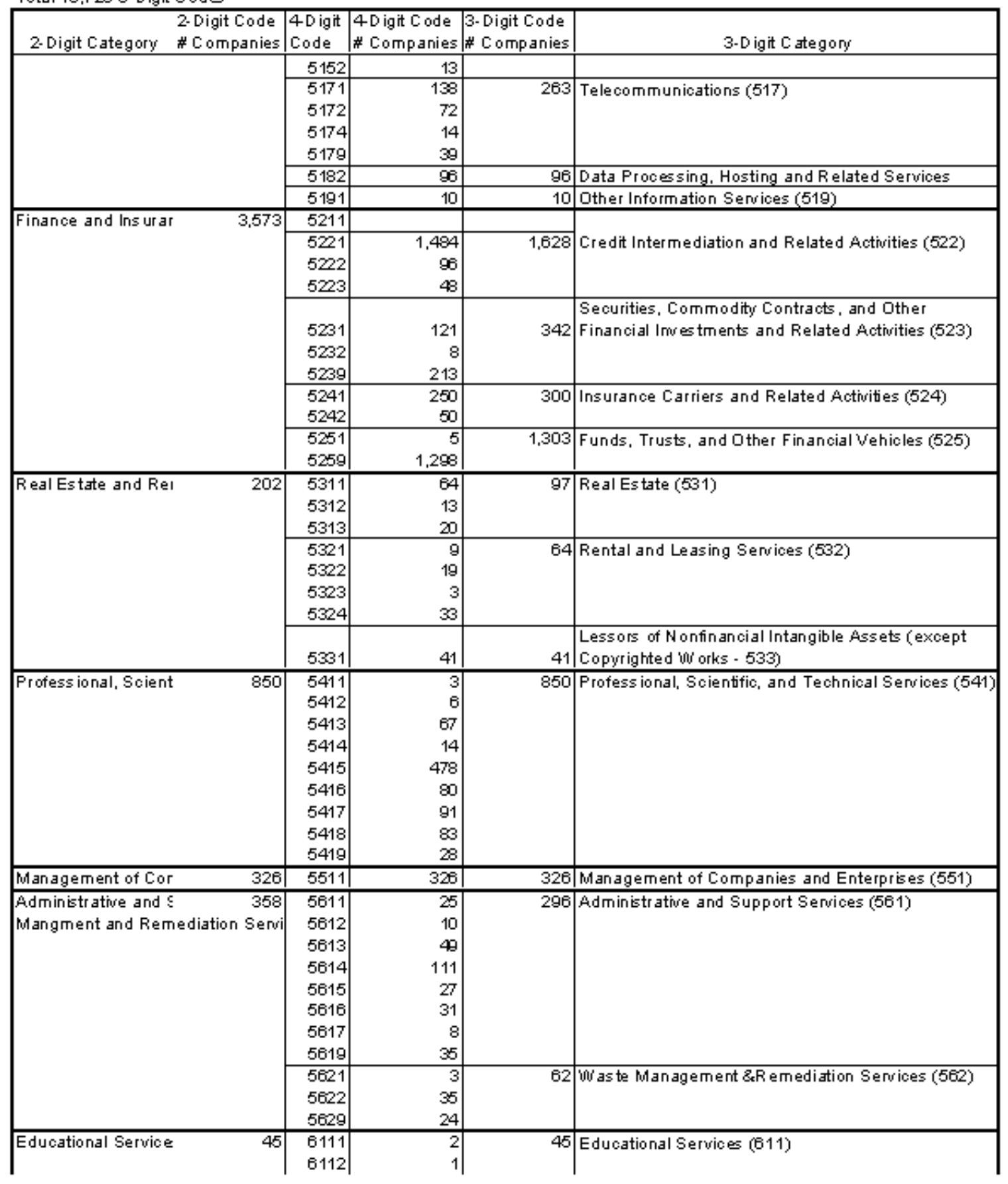


NAC: Codes:

Publicly Traded Companies - Mergent Drine (11/408 - 11/1108)

US Exchange Dnty - United States - Any Index

Total 19.720 6- Digit Codes

\begin{tabular}{|c|c|c|c|c|}
\hline $\begin{array}{ll} & \text { 2-Digit Code } \\
\text { 2-Digit Category } & \text { \# Companies } \\
\end{array}$ & \begin{tabular}{|l|}
40 igit \\
Code
\end{tabular} & $\begin{array}{l}\text { 4Digit Code } \\
\text { + Companies } \\
\end{array}$ & \begin{tabular}{|l|} 
3- Digit Code \\
\# Companies
\end{tabular} & 3-Digit C ategory \\
\hline & $\begin{array}{l}6113 \\
6114 \\
6115 \\
8116 \\
6117\end{array}$ & $\begin{array}{r}8 \\
6 \\
10 \\
10 \\
8\end{array}$ & & \\
\hline \multirow[t]{4}{*}{ Health L: are and $S 0$} & $\begin{array}{l}6211 \\
6212 \\
6213 \\
6214 \\
6215 \\
6216 \\
6219\end{array}$ & $\begin{array}{r}9 \\
3 \\
3 \\
37 \\
52 \\
37 \\
25\end{array}$ & 160 & Ambulatory Health Care Services (621) \\
\hline & $\begin{array}{l}6221 \\
6222 \\
6223\end{array}$ & $\begin{array}{r}21 \\
4 \\
2 \\
\end{array}$ & 27 & Hos pitals $(622)$ \\
\hline & $\begin{array}{l}6231 \\
6232 \\
6233 \\
6239\end{array}$ & $\begin{array}{r}32 \\
14 \\
2 \\
\end{array}$ & 49 & Nursing and Fesidential Care Facilities (628) \\
\hline & $\begin{array}{l}6241 \\
6242 \\
6243 \\
6244\end{array}$ & 8 & 11 & Sooial Ass Etanoe (624) \\
\hline \multirow[t]{3}{*}{ A.rt, Entertainment, } & $\begin{array}{l}7111 \\
7112 \\
7113 \\
7114 \\
7115\end{array}$ & $\begin{array}{r}4 \\
2 \\
1 \\
1\end{array}$ & 28 & $\begin{array}{l}\text { Ferorming Art, Spectator Sports, and Felated } \\
\text { Indetries (711) }\end{array}$ \\
\hline & 7121 & & & \\
\hline & $\begin{array}{ll}7 & 131 \\
7 & 132 \\
7 & 139\end{array}$ & $\begin{array}{l}10 \\
38 \\
30\end{array}$ & 82 & Amusement, Gambling, \& Feoreation Industries \\
\hline \multirow[t]{2}{*}{ Acoomodation and F } & $\begin{array}{l}7211 \\
7212 \\
7213\end{array}$ & $\begin{array}{r}65 \\
1\end{array}$ & 66 & Acoommodation (721) \\
\hline & $\begin{array}{l}7 Z 21 \\
7222 \\
7223 \\
7224\end{array}$ & $\begin{array}{r}88 \\
41 \\
8 \\
7\end{array}$ & 144 & Food Seruibes a nud D rirking Flabes (722) \\
\hline \multirow[t]{2}{*}{$\begin{array}{l}\text { Dther Servioes } \\
\text { (except Publio Admin - 81) }\end{array}$} & $\begin{array}{l}8111 \\
8112 \\
8113 \\
8114\end{array}$ & $\begin{array}{l}5 \\
6 \\
2 \\
3\end{array}$ & 16 & Fiepair and Maintenanoe $(811)$ \\
\hline & 8121 & 6) & 46 & Fersonal and Laundry Senuices (812) \\
\hline
\end{tabular}


NAIC $S$ Codes:

Fublicly Traded Companis - Mergent Online (11/408 - 11/11,08)

USExchange Only- United States - A.ny Index

Total 19.720 6-Digit Codes

\begin{tabular}{|c|c|c|c|c|c|}
\hline 2-Digit Category & $\begin{array}{l}\text { 2-Digit Code } \\
\text { \# Companies }\end{array}$ & \begin{tabular}{|l|}
40 igit \\
Code
\end{tabular} & $\mid \begin{array}{l}\text { 4Digit Code } \\
\text { H Companies }\end{array}$ & \begin{tabular}{|c|} 
3- Digit Code \\
$\mid \#$ Companies
\end{tabular} & 3-Digit c ategory \\
\hline & & $\begin{array}{l}8122 \\
8123 \\
8129\end{array}$ & $\begin{array}{r}7 \\
9 \\
24 \\
\end{array}$ & & \\
\hline & & $\begin{array}{l}8131 \\
8132 \\
8130 \\
8134 \\
8139 \\
8141\end{array}$ & $\begin{array}{l}1 \\
9\end{array}$ & 11 & $\begin{array}{l}\text { Feligious, Grantmaking. Ciric, Frofessional, and } \\
\text { Similar Drganizations (813) }\end{array}$ \\
\hline $\begin{array}{l}\text { Public } \\
\text { Administration (92) }\end{array}$ & 15 & $\begin{array}{l}9211 \\
9221 \\
9231 \\
9241 \\
9251 \\
9281 \\
9271 \\
9281\end{array}$ & $\begin{array}{l}5 \\
3 \\
1 \\
3 \\
1 \\
1 \\
1\end{array}$ & 15 & Fublic Administration (92) \\
\hline Total & 13,286 & & 13,260 & 13,286 & \multirow{6}{*}{ Note Zero Count Categories Deleted } \\
\hline $\mathrm{W} \equiv \mathrm{X}$ & 3,573 & & 1,404 & 1,628 & \\
\hline Min & 15 & & 1 & 1 & \\
\hline Count & 24 & & 289 & 87 & \\
\hline Awerage & 554 & & $4 \mathrm{6i}$ & 153 & \\
\hline Standard Deviation & 834.53 & & 134.13 & 282.22 & \\
\hline
\end{tabular}




\section{Appendix I: Random NAICS Category Start Number for Company Selection}

\begin{tabular}{|c|c|c|c|c|c|c|c|}
\hline \multirow[b]{2}{*}{$\begin{array}{l}\text { 3-Digit } \\
\text { code }\end{array}$} & \multirow[b]{2}{*}{$\begin{array}{c}\text { 1/11/2009 } \\
\text { Downloaded } \\
\text { 3-Digit Code } \\
\text { Adtive } \\
\text { \# Cormpanies }\end{array}$} & \multirow[b]{2}{*}{$\begin{array}{c}1 / 14 / 2009 \\
\text { Estimated } \\
5 \% \text { Sample } \\
\text { Wumber } \\
\text { of Cornpanies }\end{array}$} & \multirow[b]{2}{*}{$\begin{array}{c}\text { Pilot Test } \\
\text { Adtual } \\
5 \% \\
\text { Number } \\
\text { of Corn panies }\end{array}$} & \multicolumn{2}{|c|}{ Start Number } & \\
\hline & & & & $\begin{array}{c}1 / 14009 \\
\text { Pilot Test } \\
\text { Fandorm* } \\
1 \text { - } 20 \\
\text { CopyiP aste } \\
\text { as value }\end{array}$ & $\begin{array}{c}\text { 21112009 } \\
\text { Double } \\
\text { Sample } \\
\text { Size }\end{array}$ & $\begin{array}{l}\text { Process D } \\
\text { Fandorn * } \\
1 \text { - } 20 \\
\text { Fand } \\
\text { *19+1 }\end{array}$ & octurn entation \\
\hline 111 & 8 & & & 16 & 6 & 11 & 0.536697472 \\
\hline 112 & 6 & & & 4 & 14 & 5 & 0184321559 \\
\hline & & & & 14 & & 16 & 0.764015049 \\
\hline & & & & 3 & & 19 & 0.94891696 \\
\hline & & & & 5 & & 19 & 0.927196522 \\
\hline & & & & 5 & & 5 & 0.191361449 \\
\hline & & & & $\mathrm{B}$ & & 9 & 0.406593502 \\
\hline 113 & 3 & & & 5 & 15 & 15 & 0.749525228 \\
\hline & & & & 10 & & 9 & 0.411829421 \\
\hline & & & & 16 & & 6 & 0.256869796 \\
\hline 114 & 1 & & & 13 & 3 & 19 & 0.966346827 \\
\hline & & & & 14 & & 15 & 0.731506797 \\
\hline 115 & 9 & & & 12 & 2 & 20 & 0.999142892 \\
\hline & & 0 & 0 & 11 & 1 & & 0.074316858 \\
\hline & & 0 & 0 & 7 & & 14 & 0.694982015 \\
\hline 211 & 175 & 8 & 9 & 6 & 18 & 10 & 0.462230986 \\
\hline 212 & 181 & 9 & 9 & 11 & 1 & 9 & 0.428039959 \\
\hline & & 0 & 0 & 7 & & 2 & 0.057550247 \\
\hline & & 0 & 0 & 14 & & 11 & 0.510717346 \\
\hline 213 & 124 & $\mathrm{~B}$ & $\mathrm{G}$ & $\mathrm{B}$ & 16 & 5 & 0.206301447 \\
\hline 221 & 170 & 8 & 8 & 15 & 5 & 9 & 0.402904044 \\
\hline & & 0 & 0 & 16 & & B & 0.273422426 \\
\hline & & 0 & 0 & 13 & & 3 & 0.116637585 \\
\hline 236 & 33 & 1 & 2 & 9 & 19 & 9 & 0.436742446 \\
\hline & & 0 & 0 & 10 & & 9 & 0.401979349 \\
\hline 237 & 32 & 1 & 2 & 11 & 1 & 10 & 0.463368327 \\
\hline & & 0 & 0 & 18 & & 9 & 0.403025315 \\
\hline & & 0 & 0 & 5 & & 2 & 0.058109058 \\
\hline & & 0 & 0 & 19 & & 10 & 0.485186925 \\
\hline 238 & 12 & & & 1 & 11 & 7 & 0.330239601 \\
\hline & & 0 & 0 & 12 & & 9 & 0.435309834 \\
\hline & & 0 & 0 & 17 & & 3 & 0.11564967 \\
\hline & & 0 & 0 & 5 & & 2 & 0.076857506 \\
\hline 311 & 92 & 4 & 5 & 8 & 18 & 9 & 0.429972917 \\
\hline & & 0 & 0 & 15 & & 7 & 0.338229887 \\
\hline & & 0 & 0 & 5 & & 9 & 0.443573475 \\
\hline & & 0 & 0 & 18 & & 4 & 0.176279765 \\
\hline & & 0 & 0 & 6 & & 18 & 0.679994469 \\
\hline & & 0 & 0 & 18 & & 10 & 0.489973319 \\
\hline & & 0 & 0 & 12 & & 12 & 0.60369755 \\
\hline & & 0 & 0 & 11 & & 4 & 0.160895019 \\
\hline & & 0 & 0 & 3 & & 4 & 0.168006138 \\
\hline
\end{tabular}




\begin{tabular}{|c|c|c|c|c|c|c|c|}
\hline \multirow[b]{2}{*}{$\begin{array}{l}\text { 3-Digit } \\
\text { Code }\end{array}$} & \multirow[b]{2}{*}{$\begin{array}{c}\text { 1/11/2000 } \\
\text { Downloaded } \\
\text { 3-Digit Code } \\
\text { Adiwe } \\
\text { \# Corn panies }\end{array}$} & \multirow[b]{2}{*}{$\begin{array}{l}\text { 1/14/2000 } \\
\text { Estimated } \\
5 \% \text { Sample } \\
\text { Number } \\
\text { of Companies }\end{array}$} & \multirow[b]{2}{*}{$\begin{array}{c}\text { Pilot Test } \\
\text { Adual } \\
5 \% \\
\text { Number } \\
\text { of Companies }\end{array}$} & \multicolumn{2}{|c|}{ Start Number } & \\
\hline & & & & $\begin{array}{c}1 / 14 / 2009 \\
\text { Pilot Test } \\
\text { Riandom* } \\
1-20 \\
\text { CopyPaste } \\
\text { asvalue }\end{array}$ & $\begin{array}{c}\text { 2,11rong } \\
\text { Double } \\
\text { Sarmple } \\
\text { Size }\end{array}$ & $\begin{array}{l}\text { Process Do } \\
\text { Random* } \\
1-20 \\
\text { Rand } \\
* 19+1\end{array}$ & 10cum entation \\
\hline 312 & 39 & $\overline{1}$ & $\overline{2}$ & 11 & 1 & 12 & 0.567551403 \\
\hline & & 0 & 0 & 12 & & 12 & 0.594991937 \\
\hline 313 & a & & & 11 & 1 & 16 & 0.911321301 \\
\hline & & 0 & 0 & 4 & & 13 & 0.625969301 \\
\hline & & 0 & 0 & 15 & & 12 & 0.586032024 \\
\hline 314 & E & & & 11 & 1 & 16 & 0.786959165 \\
\hline & & 0 & 0 & 10 & & 5 & 0.231099978 \\
\hline 315 & 38 & 1 & 2 & 12 & 2 & 7 & 0.330055953 \\
\hline & & 0 & 0 & 16 & & 11 & 0.549261394 \\
\hline & & 0 & 0 & 17 & & 14 & 0.659768668 \\
\hline 316 & 20 & 1 & 1 & 15 & 5 & 16 & 0.905695158 \\
\hline & & 0 & 0 & 10 & & 17 & 0.861243951 \\
\hline & & 0 & 0 & 5 & & 11 & 0.500542427 \\
\hline 321 & 13 & & & 8 & 18 & 10 & 0.47672296 \\
\hline & & 0 & 0 & 7 & & 16 & 0.908654962 \\
\hline & & 0 & 0 & 19 & & 18 & 0.868960538 \\
\hline 322 & 34 & 1 & 2 & 12 & 2 & 14 & 0.679117226 \\
\hline & & 0 & 0 & 14 & & 20 & 0.99862046 \\
\hline 323 & 16 & & & 19 & 9 & 5 & 0.198046567 \\
\hline 324 & 33 & 1 & 1 & 16 & $\mathrm{E}$ & 15 & 0.720982328 \\
\hline 325 & 559 & 27 & 28 & 3 & 13 & 3 & 0.09124787 \\
\hline & & 0 & 0 & 9 & & 16 & 0.763850473 \\
\hline & & 0 & 0 & 8 & & 14 & 0.709130137 \\
\hline & & 0 & 0 & 14 & & 4 & 0.136646831 \\
\hline & & 0 & 0 & 14 & & 14 & 0.683961411 \\
\hline & & 0 & 0 & $g$ & & 10 & 0.497063516 \\
\hline & & 0 & 0 & 8 & & 13 & 0.646827794 \\
\hline 326 & 37 & 1 & 2 & 2 & 12 & 16 & 0.676233163 \\
\hline & & & & 8 & & 2 & 0.036095513 \\
\hline 327 & 25 & 1 & 1 & 14 & 4 & 7 & 0.310079336 \\
\hline & & & & 20 & & 9 & 0.442769203 \\
\hline & & 0 & 0 & 4 & & 11 & 0.541286818 \\
\hline & & 0 & - & 11 & & 12 & 0.559827441 \\
\hline & & 0 & - & 16 & & 1 & 0.014488177 \\
\hline 331 & 52 & 2 & 2 & 15 & 5 & 15 & 0.754259724 \\
\hline & & 0 & 0 & 1 & & 6 & 0.269498482 \\
\hline & & 0 & 0 & 14 & & 15 & 0.719696426 \\
\hline & & 0 & 0 & 18 & & E & 0.267926719 \\
\hline & & 0 & 0 & 16 & & 8 & 0.391394187 \\
\hline 332 & 66 & 3 & 4 & 5 & 15 & 19 & 0.953541941 \\
\hline & & & & 3 & & 14 & 0.662461536 \\
\hline & & 0 & 0 & 16 & & 9 & 0.424806218 \\
\hline & & 0 & 0 & 15 & & 12 & 0.563074404 \\
\hline & & 0 & 0 & 6 & & 9 & 0.439176806 \\
\hline & & 0 & - & 8 & & 7 & 0.32841575 \\
\hline & & 0 & - & 2 & & 20 & 0.981691603 \\
\hline
\end{tabular}




\begin{tabular}{|c|c|c|c|c|c|c|c|}
\hline & & & & Start M & imber & & \\
\hline $\begin{array}{l}\text { 3-Digit } \\
\text { code }\end{array}$ & $\begin{array}{c}\text { 1/11/2000 } \\
\text { Downloaded } \\
\text { 3-Digit Code } \\
\text { Adive } \\
\text { \# Companies }\end{array}$ & $\begin{array}{c}1 / 14 / 2009 \\
\text { Estimated } \\
5 \% \text { Sample } \\
\text { Number } \\
\text { of Companies }\end{array}$ & $\begin{array}{c}\text { Pilot Test } \\
\text { Adual } \\
5 \% \\
\text { Number } \\
\text { of Companies }\end{array}$ & $\begin{array}{l}1 / 14 / 2009 \\
\text { Pilot Test } \\
\text { Random* } \\
1-20 \\
\text { CopyPaste } \\
\text { asvalue }\end{array}$ & $\begin{array}{c}2,11,2009 \\
\text { Double } \\
\text { Sarmple } \\
\text { Size }\end{array}$ & $\begin{array}{l}\text { Process Do } \\
\text { Randorn * } \\
1-20 \\
\text { Rand } \\
\text { *19+1 }\end{array}$ & ocurnentation \\
\hline & & 0 & 0 & 12 & & 14 & 0.685668211 \\
\hline & & 0 & 0 & 19 & & 19 & 0.944179106 \\
\hline 333 & 168 & 8 & 8 & 18 & 8 & 7 & 0.327968173 \\
\hline & & 0 & 0 & 8 & & 20 & 0.977166400 \\
\hline & & 0 & 0 & 17 & & 15 & 0.747073793 \\
\hline & & 0 & 0 & 10 & & 7 & 0.308663121 \\
\hline & & 0 & 0 & 8 & & 18 & 0.89236727 \\
\hline & & 0 & 0 & 2 & & 8 & 0.349418378 \\
\hline & & 0 & 0 & 3 & & 20 & 0.990971589 \\
\hline 334 & 681 & 34 & 34 & 15 & 5 & 9 & 0.409302059 \\
\hline & & - & 0 & $\begin{array}{l}8 \\
9\end{array}$ & & $\begin{array}{l}6 \\
5\end{array}$ & $\left|\begin{array}{l}0.278323742 \\
0.232941362\end{array}\right|$ \\
\hline & & 0 & 0 & 9 & & $\mathrm{E}$ & 0.249548204 \\
\hline & & 0 & 0 & 8 & & 11 & 0.54968404 \\
\hline & & 0 & 0 & 12 & & 10 & 0.452275103 \\
\hline 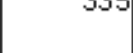 & 79 & 3 & 4 & 5 & 15 & 3 & 0.099659565 \\
\hline & & & & 15 & & 9 & 0.438793602 \\
\hline & & 0 & 0 & 1 & & 9 & 0.429999829 \\
\hline & & 0 & 0 & 14 & & 13 & 0.606234232 \\
\hline 336 & 110 & 5 & 5 & 18 & 8 & 20 & 0.978075362 \\
\hline & & 0 & 0 & 4 & & 3 & 0.084955361 \\
\hline & & 0 & 0 & 18 & & 20 & 0.977560454 \\
\hline & & 0 & 0 & 3 & & 15 & 0.762082686 \\
\hline & & 0 & 0 & 14 & & 4 & 0.182872224 \\
\hline & & 0 & 0 & 8 & & 9 & 0.442262294 \\
\hline & & 0 & 0 & 13 & & 16 & 0.785162185 \\
\hline 337 & 20 & 1 & 1 & 12 & 2 & 18 & 0.871591012 \\
\hline & & & & 18 & & 18 & 0.914606081 \\
\hline & & 0 & 0 & 2 & & 17 & 0.862679088 \\
\hline 339 & 189 & 9 & 9 & 13 & 3 & 3 & 0.099643306 \\
\hline & & 0 & 0 & E & & 13 & 0.608621056 \\
\hline 423 & 140 & 7 & 7 & 5 & 15 & 13 & 0.657017386 \\
\hline & & 0 & 0 & 13 & & 16 & 0.772536423 \\
\hline & & 0 & 0 & 1 & & 11 & 0.54483408 \\
\hline & & 0 & 0 & 11 & & 12 & 0.577997722 \\
\hline & & 0 & 0 & 14 & & 15 & 0.73377042 \\
\hline & & 0 & 0 & 18 & & 15 & 0.746669435 \\
\hline & & 0 & 0 & 12 & & 10 & 0.492529055 \\
\hline & & 0 & 0 & 20 & & 15 & 0.75941756 \\
\hline & & 0 & 0 & 12 & & 1 & 0.013738312 \\
\hline 424 & 63 & 3 & 3 & 17 & 7 & 12 & 0.584308674 \\
\hline & & 0 & 0 & 13 & & 9 & 0.397107958 \\
\hline & & 0 & 0 & 8 & & 8 & 0.384574686 \\
\hline & & 0 & 0 & 5 & & 16 & |0.799759904| \\
\hline
\end{tabular}




\begin{tabular}{|c|c|c|c|c|c|c|c|}
\hline & & & & Start id & mber & & \\
\hline $\begin{array}{l}\text { 3-Digit } \\
\text { Code }\end{array}$ & $\begin{array}{c}\text { 1/11/2009 } \\
\text { Dowriloaded } \\
\text { 3-Digit Code } \\
\text { Adiwe } \\
\text { H Companies }\end{array}$ & $\begin{array}{l}1 / 14 / 2009 \\
\text { Estimated } \\
5 \% \text { Sample } \\
\text { Number } \\
\text { af Companies }\end{array}$ & $\begin{array}{c}\text { Pilot Test } \\
\text { Adtual } \\
5 \% \\
\text { Number } \\
\text { of Companies }\end{array}$ & $\begin{array}{c}\text { 1/14/2009 } \\
\text { Pilot Test } \\
\text { Random* } \\
1 \text { - 20 } \\
\text { Copyifaste } \\
\text { asvalue }\end{array}$ & $\begin{array}{c}2,11 / 2009 \\
\text { Douple } \\
\text { Sample } \\
\text { Size }\end{array}$ & \begin{tabular}{|l|} 
Frocess Do \\
Fandorn * \\
$1-20$ \\
Fand \\
* $19+1$
\end{tabular} & ocurn entation \\
\hline & & 0 & 0 & 10 & & 2 & 0.036085854 \\
\hline & & 0 & 0 & 14 & & 4 & 0.177927906 \\
\hline & & 0 & 0 & 3 & & 17 & 0.849491615 \\
\hline & & 0 & 0 & 1 & & 12 & 0.567515815 \\
\hline & & 0 & 0 & 11 & & 7 & 0.329820269 \\
\hline 425 & 2 & & & 15 & 5 & 12 & 0.557356032 \\
\hline 441 & 32 & 1 & 2 & 7 & 17 & 4 & 0.172816971 \\
\hline & & 0 & 0 & 13 & & 19 & 0.928438239 \\
\hline & & 0 & 0 & 19 & & 16 & 0.766308453 \\
\hline 442 & 8 & & & 15 & 5 & 7 & 0.338124648 \\
\hline & & 0 & 0 & 13 & & 13 & 0.607576755 \\
\hline 443 & 12 & & & 16 & 6 & 4 & 0.13325479 \\
\hline & & 0 & 0 & 11 & & $E$ & 0.255604844 \\
\hline & & 0 & 0 & 12 & & 12 & 0.573116566 \\
\hline 445 & 17 & & & 17 & 7 & 18 & 0.874981548 \\
\hline & & 0 & 0 & 9 & & 9 & 0.43316262 \\
\hline & & 0 & a & 12 & & 16 & 0.81457636 \\
\hline 446 & 25 & 1 & 1 & 12 & 2 & 13 & 0.655593346 \\
\hline 447 & 3 & & & 1 & 11 & 13 & 0.611987597 \\
\hline 446 & 61 & 3 & 3 & 5 & 15 & 6 & 0.27812474 \\
\hline & & 0 & 0 & 11 & & 2 & 0.052523773 \\
\hline & & 0 & 0 & 3 & & 11 & 0.508373371 \\
\hline 451 & 19 & & & 14 & 4 & 8 & 0.384190068 \\
\hline & & a & 0 & 3 & & 5 & 0.211915026 \\
\hline 452 & 25 & 1 & 2 & 4 & 14 & 11 & 0.535155318 \\
\hline & & 0 & a & 4 & & 6 & 0.260502692 \\
\hline 453 & 19 & & & 7 & 17 & 10 & 0.463592581 \\
\hline & & 0 & 0 & 5 & & 7 & 0.322730907 \\
\hline & & 0 & 0 & 18 & & 15 & 0.714407959 \\
\hline & & 0 & 0 & 4 & & 15 & 0.746154574 \\
\hline 454 & 28 & 1 & 1 & 12 & 2 & 13 & 0.618374859 \\
\hline & & 0 & 0 & 16 & & 4 & 0.168763454 \\
\hline & & - & 0 & 18 & & 14 & 0.702971978 \\
\hline 461 & 25 & 1 & 2 & 1 & 11 & 13 & 0.612452627 \\
\hline & & 0 & 0 & 16 & & 12 & 0.569853525 \\
\hline 482 & 7 & & & 16 & 6 & 1 & 0.010910478 \\
\hline 483 & 15 & & & 14 & 4 & 2 & 0.048039372 \\
\hline & & 0 & 0 & 7 & & 7 & 0.296353413 \\
\hline 484 & 24 & 1 & 1 & 5 & 15 & 18 & 0.910975476 \\
\hline & & 0 & 0 & E & & 10 & 0.465606591 \\
\hline 40.0 & 1 & & & 13 & 3 & 12 & 0.598125993 \\
\hline & & 미 & 0 & 1 & & 12 & 0.560374779 \\
\hline & & 미 & 0 & 19 & & 15 & 0.724849857 \\
\hline
\end{tabular}




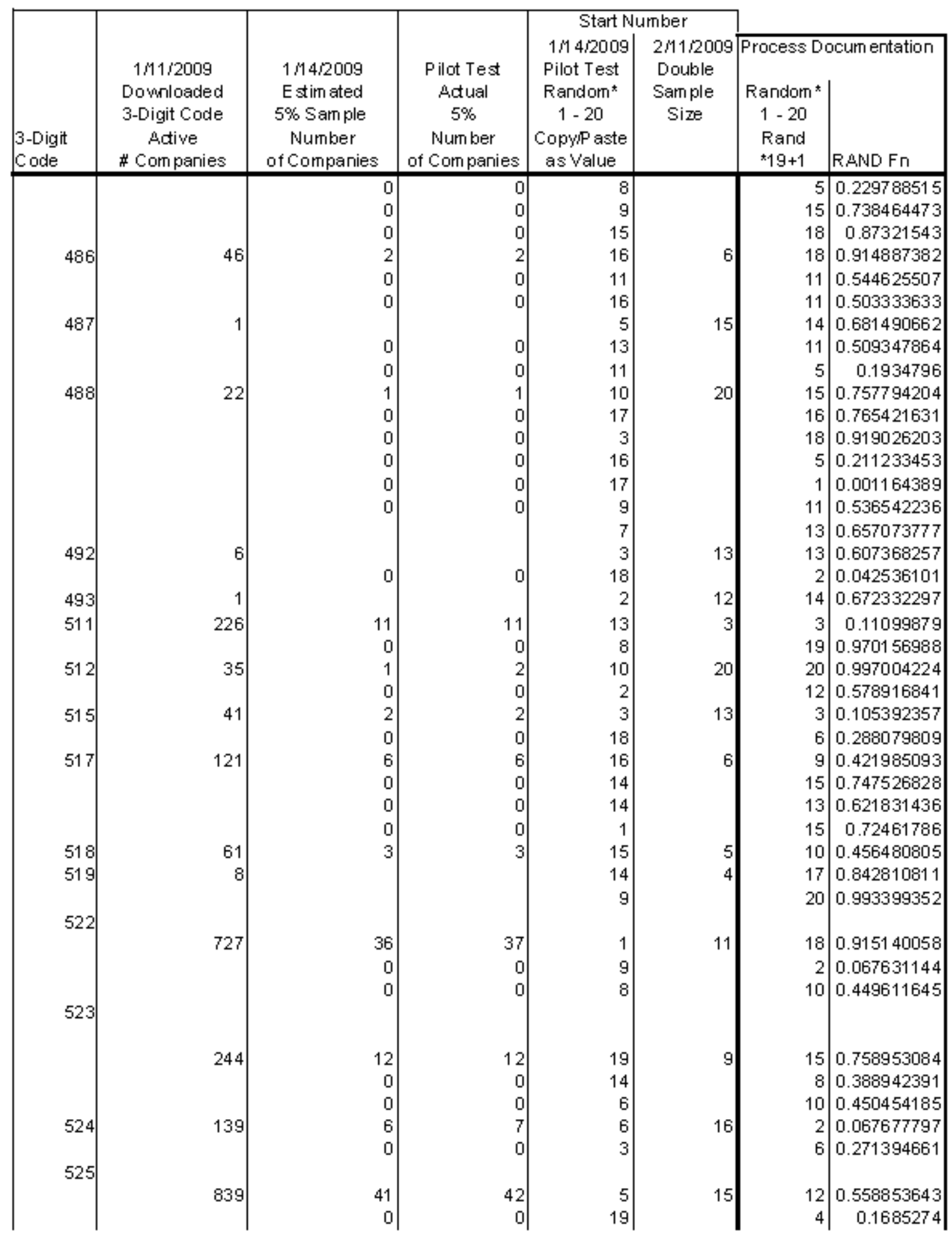




\begin{tabular}{|c|c|c|c|c|c|c|c|}
\hline & & & & Start N & mber & & \\
\hline $\begin{array}{l}\text { 3-Digit } \\
\text { Code }\end{array}$ & $\begin{array}{c}\text { 1/11/2009 } \\
\text { Downloaded } \\
\text { 3-Digit Code } \\
\text { Antive } \\
\text { \# Companies }\end{array}$ & $\begin{array}{c}1 / 14 / 2009 \\
\text { Estirn ated } \\
5 \% \text { Sample } \\
\text { Number } \\
\text { of Companies }\end{array}$ & $\begin{array}{c}\text { Pilot Test } \\
\text { Adual } \\
5 \% \\
\text { Num ber } \\
\text { of Corn panies }\end{array}$ & $\begin{array}{c}1 / 142009 \\
\text { Filot Test } \\
\text { Random* } \\
1 \text { - 20 } \\
\text { CopyPaste } \\
\text { as Value }\end{array}$ & $\begin{array}{c}2,112009 \\
\text { Double } \\
\text { Sarnple } \\
\text { Size }\end{array}$ & $\begin{array}{l}\text { Process Do } \\
\text { Randorm * } \\
1-20 \\
\text { Rand } \\
* 19+1\end{array}$ & acum entation \\
\hline 531 & 49 & 2 & 3 & 2 & 12 & 20 & 0.999939603 \\
\hline & & 0 & 0 & 11 & & 19 & 0.970252847 \\
\hline & & 0 & 0 & 14 & & 19 & 0.956506154 \\
\hline 532 & 32 & 1 & 1 & 16 & E & 2 & 0.041712358 \\
\hline & & 0 & ㅁ. & 18 & & 16 & 0.785725584 \\
\hline & & - & 미 & 13 & & 18 & 0.885207637 \\
\hline & & 0 & 0 & 14 & & 6 & 0.240330204 \\
\hline 5.5 & 24 & 1 & 1 & 14 & 4 & 10 & 0.478395062 \\
\hline 541 & 389 & 19 & 19 & 12 & 2 & 10 & 0.479945862 \\
\hline & & 0 & 0 & 7 & & 5 & 0.21754372 \\
\hline & & - & 미 & 13 & & 13 & 0.644693326 \\
\hline & & 0 & 0 & 16 & & 8 & 0.364669893 \\
\hline & & 0 & 0 & 13 & & 2 & 0.029749766 \\
\hline & & 0 & 0 & 3 & & 20 & 0.992277231 \\
\hline & & 0 & 0 & 15 & & 4 & 0.157510180 \\
\hline & & 0 & 0 & 19 & & 4 & 0.164477611 \\
\hline & & 0 & 0 & 15 & & 18 & 0.914290163 \\
\hline 551 & & 5 & Eil & 17 & 7 & 18 & $0 \operatorname{lng} 381749$ \\
\hline 561 & $\begin{array}{l}116 \\
182\end{array}$ & 9 & 9 & 12 & 2 & 5 & 0.185115228 \\
\hline & & & & 17 & & 4 & 0.132499288 \\
\hline & & 0 & 0 & 17 & & 11 & 0.527466316 \\
\hline & & 0 & 0 & 8 & & 7 & 0.301445833 \\
\hline & & 0 & 0 & 2 & & 7 & 0.309637558 \\
\hline & & 0 & 0 & 13 & & 12 & 0.56396644 \\
\hline & & 0 & 0 & 4 & & $\mathrm{E}$ & 0.266331094 \\
\hline & & 0 & 0 & 7 & & 7 & 0.305696103 \\
\hline 562 & 32 & 1 & 1 & 16 & 6 & 19 & 0.9601029246 \\
\hline & & 0 & 0 & 19 & & 2 & 0.040279825 \\
\hline & & 0 & 0 & 8 & & 15 & 0.72509426 \\
\hline 611 & 29 & 1 & 1 & 20 & 10 & 19 & 0.947791655 \\
\hline & & 0 & 0 & 10 & & 15 & 0.745351493 \\
\hline & & 0 & 0 & 9 & & 7 & 0.326974798 \\
\hline & & 0 & 0 & 2 & & 19 & 0.935474959 \\
\hline & & 0 & 0 & 17 & & 15 & 0.722461052 \\
\hline & & 0 & 0 & 10 & & 1 & 0.403770033 \\
\hline & & 0 & 0 & 6 & & 7 & 0.290990659 \\
\hline 621 & 76 & 3 & 4 & 10 & 20 & 15 & 0.722396755 \\
\hline & & 0 & 0 & 3 & & 12 & 0.585861601 \\
\hline & & 0 & 0 & a & & 12 & 0.555557874 \\
\hline & & 0 & - & 14 & & 13 & 0.608384887 \\
\hline & & 0 & 0 & 15 & & 17 & 0.827984041 \\
\hline & & 0 & 0 & 16 & & 14 & 0.68495479 \\
\hline & & 0 & 0 & 19 & & 17 & 0.841303071 \\
\hline
\end{tabular}




\begin{tabular}{|c|c|c|c|c|c|c|c|}
\hline \multirow[b]{2}{*}{$\begin{array}{l}3 \text {-Digit } \\
\text { code }\end{array}$} & \multirow[b]{2}{*}{$\begin{array}{c}\text { 1/11/2009 } \\
\text { Downloaded } \\
\text { 3-Digit Code } \\
\text { Adive } \\
\text { \# Comparies }\end{array}$} & \multirow[b]{2}{*}{$\begin{array}{c}1 / 142009 \\
\text { E stim ated } \\
5 \% \text { Sample } \\
\text { Number } \\
\text { of Companies }\end{array}$} & \multirow[b]{2}{*}{$\begin{array}{c}\text { Pilot Test } \\
\text { Adual } \\
5 \% \\
\text { Num ber } \\
\text { of Com paries }\end{array}$} & \multicolumn{2}{|c|}{ Start Nurnber } & \\
\hline & & & & $\begin{array}{c}1 / 14 i 2009 \\
\text { Pilot Test } \\
\text { Random* } \\
1-20 \\
\text { Copypaste } \\
\text { as Value }\end{array}$ & $\begin{array}{c}2 \text { 11 12009 } \\
\text { Double } \\
\text { Sample } \\
\text { Size }\end{array}$ & $\begin{array}{l}\text { Process Do } \\
\text { Fandorm * } \\
1-20 \\
\text { Fand } \\
\text { *19+1 }\end{array}$ & Oc:ulT entation \\
\hline 622 & 13 & & & 9 & 19 & 4 & 0.137131374 \\
\hline & & [] & 0 & 8 & & $\mathrm{~B}$ & 0.266620927 \\
\hline & & 0 & 0 & 7 & & 7 & 0.299948577 \\
\hline 623 & 17 & & & 19 & 9 & 10 & 0.463923708 \\
\hline & & 0 & 0 & 9 & & 19 & 0. .96432466 \\
\hline & & 0 & 0 & 18 & & 12 & 0.587222557 \\
\hline & & 0 & 0 & 8 & & 4 & 0.179915064 \\
\hline 624 & 2 & & & 18 & 8 & 18 & 0.671801069 \\
\hline & & 0 & 0 & 3 & & 11 & 0.503581597 \\
\hline & & 0 & 0 & 15 & & 15 & 0.716114866 \\
\hline & & 0 & 0 & 8 & & 15 & 0.726258506 \\
\hline 711 & & & & & & & \\
\hline & 17 & & & $\mathrm{~B}$ & 16 & 20 & 0.975093729 \\
\hline & & 0 & 0 & 9 & & 17 & 0.848207618 \\
\hline & & 0 & 0 & 15 & & 14 & 0.6633454 \\
\hline & & 0 & 0 & 15 & & 9 & 0.399721101 \\
\hline & & 0 & 0 & 8 & & 16 & 0.797431819 \\
\hline & & 0 & 0 & 17 & & 6 & 0.281309713 \\
\hline 713 & 51 & 2 & 2 & 19 & 9 & 5 & 0.219481571 \\
\hline & & 0 & 0 & 4 & & 7 & 0.339763221 \\
\hline & & 0 & 0 & 15 & & 14 & 0.708681111 \\
\hline 721 & 25 & 1 & 2 & 4 & 14 & 12 & 0.575408411 \\
\hline & & 0 & 0 & 7 & & 8 & $0.39444407 \mathrm{~B}$ \\
\hline & & 0 & 0 & 17 & & 18 & 0.877974716 \\
\hline 722 & 71 & 3 & 4 & 4 & 14 & 16 & 0.607299635 \\
\hline & & 0 & 0 & 13 & & $\mathrm{~B}$ & 0.262252373 \\
\hline & & 0 & 0 & 1 & & 17 & 0.847966612 \\
\hline & & 0 & 0 & 13 & & 14 & 0.687601481 \\
\hline & & 0 & 0 & 12 & & 10 & 0.472330554 \\
\hline & & 0 & 0 & 6 & & 10 & 0.462131079 \\
\hline & & 0 & 0 & 8 & & 11 & 0.545725619 \\
\hline & & 0 & 0 & 5 & & 2 & 0.06448516 \\
\hline & & 0 & 0 & 3 & & 4 & 0.180789657 \\
\hline 811 & 8 & & & 2 & 12 & 1 & 0.024927115 \\
\hline & & 0 & 0 & 3 & & 11 & 0.538943163 \\
\hline & & 0 & 0 & 4 & & 18 & 0.900070707 \\
\hline & & 0 & 0 & 14 & & 8 & 0.368467495 \\
\hline 612 & 30 & 1 & 2 & 6 & 16 & 12 & 0.599305006 \\
\hline & & 0 & 0 & 19 & & $\mathrm{~B}$ & 0.239478593 \\
\hline & & 0 & 0 & 7 & & 11 & 0.54549091 \\
\hline & & 0 & 0 & 10 & & 17 & 0.851779681 \\
\hline 813 & & & & & & 4 & \\
\hline & 9 & & & 15 & 5 & 11 & 0.519847239 \\
\hline & & 0 & 0 & 11 & & $\mathrm{~B}$ & 0.285734241 \\
\hline & & 0 & 0 & 4 & & 4 & $0.162975056 \mid$ \\
\hline
\end{tabular}




\begin{tabular}{|c|c|c|c|c|c|c|c|}
\hline \multirow[b]{2}{*}{$\begin{array}{l}\text { 3-Digit } \\
\text { Code }\end{array}$} & \multirow[b]{2}{*}{$\begin{array}{c}\text { 111/2009 } \\
\text { Dovnloaded } \\
\text { 3-Digit Code } \\
\text { Adtive } \\
\text { H Corn panies }\end{array}$} & \multirow[b]{2}{*}{$\begin{array}{c}1,14 / 2009 \\
\text { E sirmated } \\
5 \% \text { Sample } \\
\text { Wumber } \\
\text { of Companies }\end{array}$} & \multirow[b]{2}{*}{$\begin{array}{c}\text { Pilot Test } \\
\text { Adtual } \\
5 \% \\
\text { Number } \\
\text { of Cormpanies }\end{array}$} & \multicolumn{2}{|c|}{ Start Number } & & \\
\hline & & & & $\begin{array}{c}1 / 14 / 2009 \\
\text { Pilot Test } \\
\text { Random* } \\
1-20 \\
\text { Copyipaste } \\
\text { asvalue }\end{array}$ & $\begin{array}{l}\text { 2/112000 } \\
\text { Double } \\
\text { Sample } \\
\text { Size }\end{array}$ & $\begin{array}{l}\text { Frocess D } \\
\text { Fandorn * } \\
1-20 \\
\text { Rand } \\
* 19+1\end{array}$ & OCLNT entation \\
\hline 920 & 10 & c & 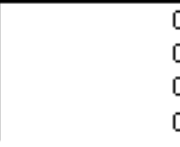 & $\begin{array}{r}11 \\
11 \\
9 \\
6\end{array}$ & 16 & $\begin{array}{r}20 \\
10 \\
8 \\
8 \\
\end{array}$ & $\begin{array}{l}0.974255015 \\
0.477587187 \\
0.368361481 \\
0.378275266\end{array}$ \\
\hline
\end{tabular}

\begin{tabular}{|c|r|r|r|}
\hline Total & 7,268 & 325 & 349 \\
\hline Max & 839 & \\
Min & 1 \\
\hline Count & 87 \\
\hline Bferage & 84 \\
\hline Std.Dev. & 153.46 \\
\hline
\end{tabular}




\section{Appendix J: Pilot Test Email Campaign}

Survey e-mail Campaign model based on Dillman, D. (2000). Mail and Internet Surveys: The Tailored Design Method (2nd ed.). New York: John Wiley \& Sons, Inc. (pp. $156-$ 185).

Scheduled as follows:

Week 1 -- (02/24/09) Tue 1st Contact Email Prior Letter

Week 1 -- (02/27/09) Fr 2nd Contact Survey Cover Letter

Week 3 -- (03/09/09) Mon 3rd Contact E-mail Thank You/Reminder

Week 5 -- (03/23/09) Mon 4th Contact Repeat Questionnaire

Week 10 -- (04/27/09) Mon 5th Final Contact

\section{$1^{\text {st }}$ Contact Email prior letter}

Date: $\quad 2 / 24 / 09$

To: $\quad$ Contact, Title

From: $\quad$ Robert Fowke, Portland State University

Subject: $\quad$ Ph.D. Dissertation Survey

Your company is one of a set of publicly traded companies that has been selected to participate in a brief survey regarding performance measures for managerial decision making, in partial fulfillment of the requirements for a Ph.D. in Systems Science: Business Administration. Multiple participants, who will remain anonymous, are welcome from each selected company (coded for anonymity).

Within the next couple of days you will be receiving the brief survey from this same email address. We would greatly appreciate it if you could take a few moments to complete it. By doing so you will help ensure that we have the best information possible. If you have any questions, or if a different contact should be used for the email survey distribution, please advise rfowke@pdx.edu using Company Code 9999.999.

Thank you in advance for your cooperation.

Sincerely,

Robert Fowke, Ph.D. Candidate

Portland State University 


\section{$2^{\text {nd }}$ Contact Survey Cover Letter}

Date: $\quad 2 / 27 / 09$

To: $\quad$ Contact, Title

Company

From: $\quad$ Robert Fowke

Subject: $\quad$ Ph.D. Dissertation Survey Request

As mentioned previously your company is one of set of publicly traded companies that has been selected to participate in a brief 12 question survey regarding performance measures in partial fulfillment of the requirements for a Ph.D. in Systems Science: Business Administration.

This study accommodates responses from a variety of job functions and departments. Multiple participants, who will remain anonymous, are therefore welcome from each selected company. Please ask interested participants to use company code 9999.999 when completing this questionnaire to assure company anonymity.

[Insert survey link]

Results of the survey will be made available to participants upon request.

Thank you in advance for your consideration and participation.

Sincerely,

Robert Fowke, Ph.D. Candidate

Portland State University 


\section{$3^{\text {rd }}$ Contact E-mail thank you/reminder}

Date: $\quad 3 / 9 / 09$

To: $\quad$ Contact, Title

Company

From: $\quad$ Robert Fowke

Subject: $\quad$ Ph.D. Dissertation Survey Request

About a week ago we sent you a survey via e-mail. Your company was randomly selected from a set of publicly traded companies for participation.

If you have already completed the questionnaire, please accept our sincere thanks. If not, please do so today using Company Code 9999.999. The questionnaire is a very brief 12 question questionnaire that will help define synergies in multi-attribute performance measurement systems. If additional personnel in your company would like to participate please ask them to do so using the same Company Code 9999.999.

Please find the survey link included in this message for your convenience:

[insert survey link]

Thank you in advance for your consideration and participation.

Sincerely,

Robert Fowke, Ph.D. Candidate

Portland State University 


\section{$4^{\text {th }}$ Contact Repeat Questionnaire}

Date: $\quad 3 / 23 / 09$

To: $\quad$ Contact, Title

Company

From: $\quad$ Robert Fowke

Subject: $\quad$ Ph.D. Dissertation Survey Request

About three weeks ago I sent you a brief 12 question survey request about your company's use of performance measurements. To the best of our knowledge, no one from your company has yet participated.

The comments of people who have already responded include a wide variety of measurement systems. We think the results are going to be very useful to define synergies in multi-attribute performance measurement systems.

We are writing again because of the importance that your questionnaire has for helping to get accurate results. Although we sent questionnaires to a random selection of publicly traded companies, it's only by hearing from nearly everyone in the sample that we can be sure that the results are truly representative.

A few people have written to advise that they are not the appropriate company contact. If this is the case, please advise rfowke@pdx.edu of the best contact for your company (Company Code 9999.999).

A comment on our survey procedures: Individual names are not included in the survey response, and so remain anonymous. As noted above, the company is also coded for confidentiality. Protecting the confidentiality of people's answers is very important to us, as well as the University.

We hope you will fill out and return the questionnaire soon. Please find the survey link included in this message for your convenience:

[insert survey link]

Sincerely,

Robert Fowke, Ph.D. Candidate

Portland State University

P. S. If you have concerns or problems about your participation in this study or your rights as a research subject, please contact the Human Subjects Research Review Committee, Office of Research and sponsored Projects, 111 Cramer Hall, Portland State University, (503) 725-4288 / 1-877-480-4400. If you have questions about the study itself, contact Robert Fowke at Portland State University, c/o Dr. Beverly Fuller, SBA. 


\section{$5^{\text {th }}$ Final Contact}

Date: $\quad 4 / 27 / 09$

To: $\quad$ Contact, Title

Company

From: $\quad$ Robert Fowke

Subject: $\quad$ Ph.D. Dissertation Survey Request

During the last two months we have sent you several email requests about a research study we are conducting in partial fulfillment of the requirements for a Ph.D. in Systems Science: Business Administration.

The purpose is to help define synergies in multi-attribute performance measurement systems among publicly traded companies.

The study is drawing to a close and this is the last contact that will be made to the random selection of companies.

We are sending this final contact because of our concern that people who have not responded may utilize different performance measurement systems than those who have responded. Hearing from everyone in this small sample survey helps assure that the survey results are as accurate as possible.

We also want to assure you that your response to this study is voluntary, and if you prefer not to respond that is fine.

Finally, we appreciate your willingness to consider our request as we conclude this effort to better understand performance measurement system synergies in publicly traded companies.

We hope you will take the opportunity fill out and submit the brief 12 question questionnaire using Company code 9999.999:

[insert survey link]

Sincerely,

Robert Fowke, Ph.D. Candidate

Portland State University 


\section{Appendix K: Dissertation Survey Email Campaign}

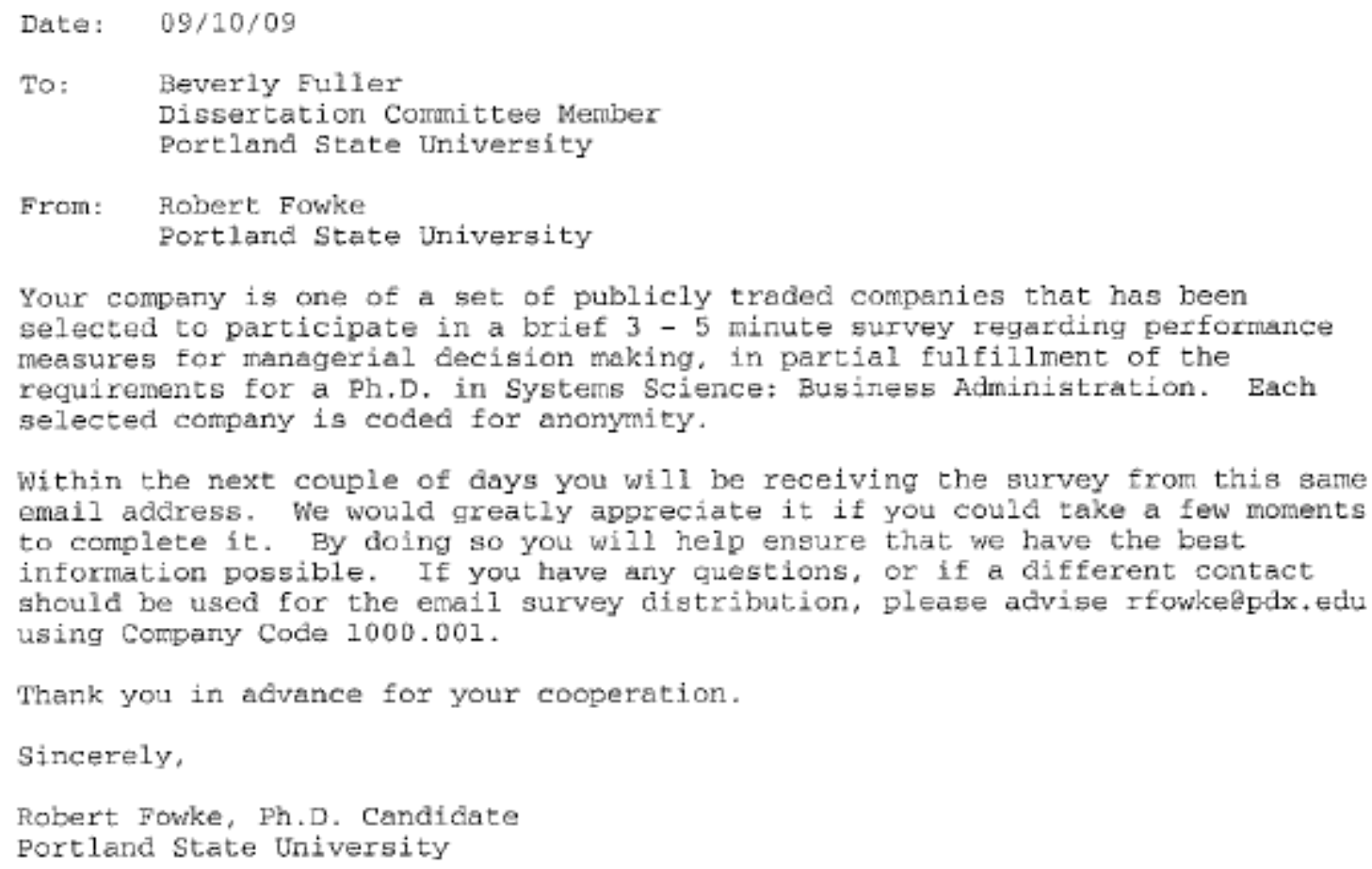


Date: $09 / 14 / 09$

To: Beverly Fuller

Dissertation Committee Member

Portland State University

From: Robert Fowke

Portland State university

As mentioned previously your company is one of a set of publicly traded companies that has been selected to participate in a brief $3-5$ minute, 12 question survey regarding performance measures in partial fulfillment of the requirements for a $\mathrm{Ph} . \mathrm{D}$. in Systems Science: Business Administration.

This study accommodates responses from a variety of industries, job functions, and departments. Selected companies are coded for anonynity and participants will remain anonymous. Please use company code 1000.001 when completing this questionnaire to assure company anonymity.

http://survey.oit.pex. edu/ss/1.A11/JGsB694B5E81WzD9U27673J.htm

Results of the survey will be made available to participants upon request. If you have any questions, or if a different contact should be used for the email survey distribution, please advise rfowkelpdx.edu using company code 1000.001.

For questions regarding the validity of this study, please contact the Human Subjects Research Review Committee, Office of Research and Sponsored Projects, 600 Unitus Bldg., Fortland State university, (503) 725-4288 / 1-877-480-4400. Refer to HSSRC Proposal \# 08755.

Thank you in advance for your consideration and partieipation.

Sincerely,

Robert Fowke, Ph.D. Candidate

portland State University 
Date: 09/21/09

To: Beverly Fuller

Dissertation Committee Nember

Portland State University

From: Robert Fowke

Portland State university

About a week ago we sent you a survey via e-mail. Your company was randonly selected from a set of publicly traded companies for participation.

If you have already completed the questionnaire, please accept our sincere thanks. If not, please do so today using Company code 1000.001. The questionnaire is a very brief 3 - 5 minute, 12 question guestionnaire that will help define synergies in multi-attribute performance measurement systems. If someone else in your company would be the appropriate person to complete the questionnaire, please ask them to do so using the ame Company Code 1000,001 .

please find the survey link included in this message for your convenience:

http://survey.oit.pdx. edu/ss/1.d11/JGsB694B5F91W2D9U27674J.htm

For questions regarding the validity of this study, please contact the Human Subjects Research Review Committee, Office of Research and Sponsored Projects, 600 unitus Bldg., Portland State University, (503) 725-4288/1-877-480-4400. Refer to HSRRC Proposal \# 08755.

Thank you in advance for your consideration and participation.

Sincerely,

Robert Fowke, Ph.D. Candidate

Portland State University 


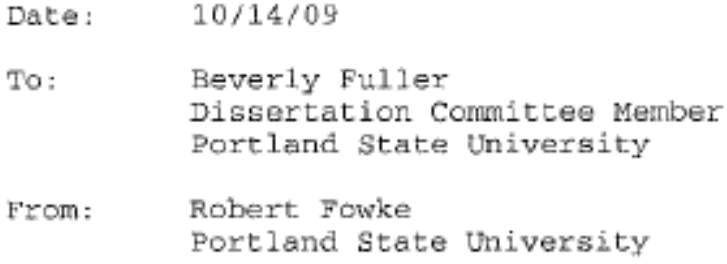

About four weeks ago I sent you a brief 3 -5 minute, 12 guestion survey about your company's use of performance measurements. To the best of our knowledge no one from your company has yet participated.

The comments of people who have already responded include a wide variety of measurement systems. We think the results are going to be very useful to define synergies in multi-attribute performance measurement systems.

We are writing again because of the importance that your questionnaire has for helping to get accurate results. Although we sent questionnaires to a random selection of publicly traded companies, it's only by hearing from nearly everyone in the sample that we can be sure that the results are truly representative.

A few people have written to advise that they are not the appropriate company contact. If this is the case, please advise rfowkedpdx. edu of the best contact for your company (Company Code 1000.001).

A comment on our survey procedures: Individual names are not included in the survey response, and so remain anonymous. As noted above, the company is also coded for confidentiality. Protecting the confidentiality of people's answers is very important to us, as well as the University.

We hope you will fill out and return the questionnaire soon. Please find the survey link included in this message for your convenience:

http://survey. oit.pdx, edu/ss/l,dl1/JGaB694B5701bizD9U27575J.htm

Sincerely.

Robert Fowke, Ph.D. Candidate

Portland State University

P. S. If you have concerns or problems about your participation in this study or your rights as a research subject, please contact the Human Subjects Research Review Comittee, office of Research and Sponsored grojects, 600 Unitus Bldg., Portland State University, (503) 725-4288/ 1-877-480-4400. Refer to HSRRC Proposal \# 08755. If you have questions about the study itself, contact Robert Fowke at Portland State University, c/o Dr. Beverly Fuller, SBA. 


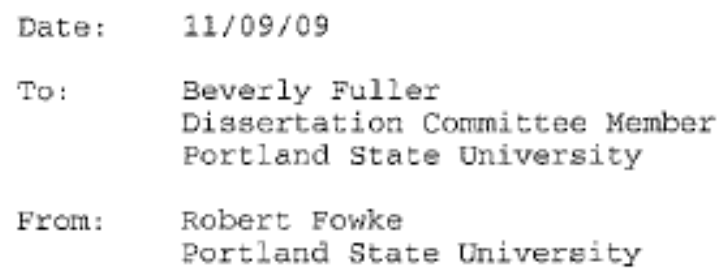

During the last two months we have sent you several email requests about a research study we are conducting in partial fulfillment of the requirements for a Ph.D. in Systems Science: Business Administration.

The purpose is to help define synergies in multi-attribute performance measurement systems among publicly traded companies.

The study is drawing to a close and this is the last contact that will be made to the random selection of companies.

We are sending this final contact because of our concern that people who have not responded thay utilize different performance measurement systems than those who have responded. Hearing from everyone in this small sample survey helps assure that the survey results are as accurate as possible.

We also want to assure you that your response to this study is voluntary, and if you prefer not to respond that is fine.

Finally, we appreciate your willingness to consider our request as we conclude this effort to better understand performance measurement system synergies in publicly traded companies.

We hope you will take the opportunity fill out and submit the brief 12 question questionnaire using Company Code 1000.001:

http: //survey .oit.pdx. edu/ss/1. d11/JGsB694C6C81wZD9U27676J.htm

For questions regarding the validity of this gtudy, please contact the Human Subjects Research Review Comittee, Office of Research and Sponsored Projects, 600 Unitus Bldg., Portland State University, (503) 725-4288/ 1-877-480-4400. Refer to HSRRC Proposal \# 08755

Sincerely,

Robert Fowke, Ph.D. Candidate

Portland State University 


\section{Appendix L: Survey Results}

\begin{tabular}{|c|c|c|c|c|c|c|}
\hline $\begin{array}{l}\text { Fiesp. } \\
\text { ID }\end{array}$ & [Date Subrnitted & IP A Átre SS & $\begin{array}{l}\text { Company } \\
\text { Code }\end{array}$ & product change & $\begin{array}{l}\text { processes } \\
\text { change }\end{array}$ & $\begin{array}{l}\text { performance } \\
\text { measurement } \\
\text { change }\end{array}$ \\
\hline 1 & $21271200911: 11$ & 70.108 .251 .167 & 3250.453 & Don't kroun & Dorin't known & Dont know \\
\hline 2 & 21271200911.56 & 209.63 .205 .1 & 3360.048 & $=3$ years & 3 years & -3years \\
\hline 3 & $21271200912: 06$ & 72.236 .149 .3 & 4230.135 & $=3$ years & 3 years & 63 years \\
\hline 4 & 21271200912.52 & 198.69 .123 .41 & 7220.054 & $=3$ years & 3 years & $<3$ years \\
\hline 5 & $3 / 2 / 20098: 14$ & 67.85 .119 .60 & 4230.135 & $=3$ years & 3years & $=10$ years \\
\hline 6 & $32200914: 46$ & 168.215 .137 .121 & 5250.475 & $=3$ years & oyears & $=3$ years \\
\hline 7 & $3 / 60098.51$ & 65.206 .42 .2 & 7130.039 & $=3$ years & 3 - 10 years & $=3$ years \\
\hline 8 & $30200911: 19$ & 64.74 .23 .10 & 8120.006 & $=3$ years & 3 - 10 years & 3y'ears \\
\hline 9 & 32420096.42 & 65.175 .139 .75 & 5410.052 & $=3$ years & $\angle 3$ y'ears & $3-10$ years \\
\hline 10 & $324 / 200911: 27$ & 64.129 .227 .4 & 5410.352 & 3 - 10years & 3 - 10 years & $3-10$ years \\
\hline 11 & 3261200915.47 & 198.160 .190 .11 & 3250.303 & $=3$ years & $=3$ years & $=3$ years \\
\hline 12 & $41 / 200915: 30$ & 156.73 .204 .228 & 2210.105 & 3 - 10years & 3 - 10 years & -3years \\
\hline 13 & 4130096.48 & 71.43152 .106 & 2370.001 & $=3$ years & 3 years & -3years \\
\hline 14 & $4127 / 200917: 25$ & 69.74 .70 .139 & 3110.038 & - 3 years & 3years & $3-10$ years \\
\hline 15 & 41281200910.56 & 67.141 .188 .233 & 3250.153 & $=3$ years & Don't know & Dont know \\
\hline 16 & $41291200914: 26$ & 63.78 .48 .130 & 3110.058 & Don't know & 3 years & 3 - 10 years \\
\hline 17 & $9114 / 200911: 30$ & 12.52 .23 .98 & 3330.104 & $=3$ years & 3 - 10 years & 3 - 10 years \\
\hline 18 & $9114200911: 43$ & 70.89 .43 .110 & 3110.084 & $=3$ years & 3 years & 63 years \\
\hline 19 & $9144200911: 43$ & 69.74 .132 .195 & 5110.159 & $=3$ years & -3years & 3years \\
\hline 20 & $9 / 14 / 200911.45$ & 204.111 .32 .126 & 5220.625 & $=3$ years & 3 years & 3 - 10 years \\
\hline 21 & $9 / 14 / 200911: 48$ & 4.21 .1 .2 & 5220.315 & $=3$ years & 3 - 10 years & $3-10$ years \\
\hline 22 & $9 / 14 / 200911.54$ & 216.185 .74 .157 & 5620.03 & $=10$ years & 3 - 10 years & $3-10$ years \\
\hline 23 & $9114 / 200911.56$ & 63.252 .73233 & 5220.349 & $=3$ years & 3 - 10 years & $=10$ years \\
\hline 24 & $9 / 14 / 200912: 47$ & 192.122 .244 .74 & 2210.101 & $=10$ years & $=3$ years & दyears \\
\hline 25 & $9 / 14 / 200912.53$ & 74.95 .96 .97 & 3120.033 & 3 - 10years & $=10$ years & $=3 \mathrm{ye}$ \\
\hline 26 & 91141200912.54 & 208.47 .77 .6 & 3340.439 & $=3$ years & 3 years & 3 - 10 years \\
\hline 27 & $9114200914: 09$ & 12.129 .83 .52 & 3220.006 & 3-10years & 3 - 10 years & 3 - 10 years \\
\hline 28 & $9 / 14 / 200914: 34$ & 207.118 .55 .15 & 3330.13 & a 3 years & 3 years & 3 - 10 years \\
\hline 29 & $9 / 14 / 200920: 04$ & 72.241 .200 .134 & 5330.016 & $=10$ years & 3 - 10 years & Don't knowh \\
\hline 30 & $915 / 20098: 03$ & 207.14 .236 .120 & 5220.319 & $=3$ years & 3 years & 63 years \\
\hline 31 & $915 / 20098: 22$ & 24.105 .177 .201 & 3330.07 & $=3$ years & 3 years & $<3$ years \\
\hline 32 & $915 / 20098.56$ & 205.149 .142 .22 & 3340.499 & 3 - 10years & 3 - 10 years & $3-10$ years \\
\hline 33 & $915 / 20099: 23$ & 198.51 .251 .199 & 3340.601 & $=3$ years & 3 years & -3years \\
\hline 34 & $915 / 2009947$ & 69.55 .155 .226 & 2210.049 & $=10$ years & $=10$ years & $>10$ years \\
\hline 35 & $91151200913: 45$ & 70.150 .142 .201 & 5220.359 & $\div 3$ years & $\Leftrightarrow 3$ y'ears & $3-10$ years \\
\hline 36 & 915000913.58 & 24.199 .194 .26 & 5240.074 & 3 - 10years & 3 - 10 years & 3 - 10 years \\
\hline 37 & $9115200914: 05$ & 216.195 .221 .162 & 5250.791 & 3 - 10years & 3 years & $3-10$ years \\
\hline 38 & $9115 / 200914.51$ & 63.64 .84 .194 & 5220.337 & $=3$ years & 3 y'ears & $>10$ years \\
\hline 39 & $9115200915: 41$ & 72.255 .60 .179 & 5220.135 & $=3$ years & 3 years & -3years \\
\hline 40 & 9115200915.58 & 160.69 .1 .242 & 3360.074 & $=3$ years & 3 years & 3years \\
\hline 41 & $916 / 20090: 03$ & 70.165 .34 .2 & 3390.087 & - 3 years & 3 - 10 years & $3-10$ years \\
\hline 42 & $9116 / 200917: 49$ & 75.144 .127 .213 & 3340.421 & 3 - 10years & 3 - 10 years & $3-10$ years \\
\hline 43 & $9117 / 20098: 40$ & 64.47 .57 .228 & 5150.099 & $=3$ years & 3 - 10 years & 3 - 10 years \\
\hline 44 & $9118000913: 23$ & 65.203 .132 .130 & 3250.277 & $3-10$ years & 3 - 10 years & 63 years \\
\hline 45 & $9 / 21 / 200911: 03$ & 66.45 .174 .194 & 3220.026 & $=10$ years & 3 years & 3 - 10 years \\
\hline 46 & $9121 / 200911: 21$ & 97.118 .129 .64 & 2370.027 & Dorit kroum & 3 - 10 years & Don't klown \\
\hline 47 & $9 / 21 / 200911.50$ & 76.112 .253 .158 & 5410.018 & $=3$ years & 3 y'ears & 3 - 10 years \\
\hline 48 & $9 / 21 / 2009$ 12:00 & 12.163 .232 .166 & 5220.199 & $=3$ years & 3 years & $3-10$ years \\
\hline
\end{tabular}




\begin{tabular}{|c|c|c|c|c|c|c|}
\hline $\begin{array}{l}\text { Fesp. } \\
\text { ID }\end{array}$ & [Date Submitted & IF Address & $\begin{array}{l}\text { Company } \\
\text { Coude }\end{array}$ & product change & $\begin{array}{l}\text { processes } \\
\text { change }\end{array}$ & $\begin{array}{l}\text { performarice } \\
\text { measurement } \\
\text { change }\end{array}$ \\
\hline 49 & $9121 / 200913: 21$ & 204.60 .84 .2 & 5220.679 & $\div 3$ years & $=3$ years & 3 - 10 years \\
\hline 50 & $9 / 21 / 200915: 11$ & 65.117 .82 .150 & 3320.007 & < 3 years & $=3$ years & 3 - 10 years \\
\hline 51 & $922 / 20092: 10$ & 82.111 .224 .229 & 2110.06 & $>10$ years & $=10$ years & 3 - 10years \\
\hline 52 & $9 / 22 / 20096.31$ & 64.198 .156 .98 & 3340.191 & $=3$ years & $=3$ years & $=3$ years \\
\hline 53 & $9 / 22 / 20096.35$ & 76.5 .100 .58 & 5220.233 & $<3$ years & $=3$ years & Dont know \\
\hline 54 & $9122200910: 42$ & 65.123 .193 .159 & 5220.449 & 3-10years & $3-10$ years & 3 - 10 years \\
\hline 55 & 9122200911.54 & 173.14 .208 .177 & 5110.019 & $\Leftrightarrow 3$ years & 63 years & -3years \\
\hline 56 & $9 / 22200913.36$ & 198.204 .43 .202 & 3330.146 & $=3$ years & $=3$ years & 3 - 10 years \\
\hline 57 & $9 / 23200911: 30$ & 209.149 .145 .162 & 5220.659 & $=3$ years & $=3$ years & $=3$ years \\
\hline 58 & $9 / 24200912: 11$ & 72.48 .145 .130 & 2210.001 & 3-10years & $=3$ years & 3 - 10 years \\
\hline 59 & 9127200919.50 & 63.125 .144 .103 & 3250.449 & 3-10years & 3 - 10 years & $=3$ years \\
\hline 60 & $928 / 2009831$ & 65.51 .35 .66 & 5610.078 & $=3$ years & 6years & 3 - 10 years \\
\hline 61 & $1014120099: 48$ & 12.48 .114 .48 & 5220.499 & $\Leftrightarrow 3$ years & 63 years & 3years \\
\hline 62 & $10 / 14 / 200910: 04$ & 146.126 .61 .241 & 2210.141 & $=10$ years & 3 - 10 years & 3years \\
\hline 63 & $10 / 14 / 2009$ 10:07 & 12.105 .95 .2 & 3260.036 & 63 years & $=3$ years & $3-10$ years \\
\hline 64 & $10 / 14 / 200911: 45$ & 206.201 .24 .250 & 5110.005 & $=3$ years & syears & 3years \\
\hline 65 & $1014200912: 41$ & 205.169 .14 .166 & 4430.006 & $=3$ years & $=3$ years & 3years \\
\hline 66 & $10141200913: 31$ & 70.182 .107 .9 & 3350.009 & $=3$ years & $=3$ years & Dont know \\
\hline 67 & $10 / 141200915: 47$ & 12.90 .233 .119 & 3340.131 & $=3$ years & $=3$ years & $=3$ years \\
\hline 68 & $10151200912: 16$ & 38.98 .145 .30 & 5410.15 & $>10$ years & $=3$ years & $=3$ years \\
\hline 69 & 101612009948 & 66.238 .13 .98 & 3250.035 & $\because 3$ years & 83 years & 3years \\
\hline 70 & $1025 / 20096: 46$ & 74.10 .239 .2 & 9999.999 & $\Leftrightarrow 3$ years & 83 years & 3 - 10 years \\
\hline 71 & $10 / 261200917: 19$ & 96.207 .37 .202 & 3340.477 & =3 years & $=3$ years & Don't know \\
\hline 72 & $11 / 4 / 20099: 03$ & 209.0 .205 .243 & 3340.229 & Don't know & Don't known & Don't know \\
\hline 73 & $11,900913.58$ & 70.184 .232 .149 & 5220.417 & $\div 3$ years & -3 years & $=3$ years \\
\hline 74 & 111112009 11:01 & 207.126 .196 .16 & 8120.028 & 3-10years & $=3$ years & 3-10years \\
\hline 75 & $11.13120097: 52$ & 36.97 .193 .114 & 2110.146 & $=10$ years & 3years & $=10$ years \\
\hline 76 & $11.13120098: 17$ & 168.220 .96 .73 & 4410.025 & 3- 10years & syears & 3years \\
\hline
\end{tabular}




\begin{tabular}{|c|c|c|c|c|}
\hline $\begin{array}{l}\text { Resp. } \\
\text { ID }\end{array}$ & position & & department & \\
\hline 1 & Executive & & Corporate & \\
\hline 2 & Executiwe & & Finance & \\
\hline 3 & \multicolumn{2}{|c|}{ Front line supervisor } & Finance & \\
\hline 4 & Executive & & Finance & \\
\hline 5 & Executive & & Corporate & \\
\hline 6 & Executive & & Finance & \\
\hline 7 & Other & Director & Finance & \\
\hline g & Executive & CEO & Copporate & \\
\hline 9 & Executive & & Corporate & \\
\hline 10 & $\mathrm{VP}$ & & Corporate & \\
\hline 11 & Other & Fublic Relations & Corporate & \\
\hline 12 & Other & Director & Corporate & \\
\hline 13 & Executive & & Corporate & \\
\hline 14 & $\mathrm{VP}$ & & $\mathrm{HR}$ & \\
\hline 15 & Executive & & Corporate & \\
\hline 16 & $\mathrm{VP}$ & & Corporate & \\
\hline 17 & $\mathrm{VF}$ & & Finance & \\
\hline 18 & Executiwe & & Corporate & \\
\hline 19 & Exerutive & & Corporate & \\
\hline 20 & Executiwe & & Finance & \\
\hline 21 & Executive & & Corporate & \\
\hline 22 & Executive & & Corporate & \\
\hline 23 & $\mathrm{VP}$ & & Finance & \\
\hline 24 & Other & | rwestor Relations officer & Finance & \\
\hline 25 & Executive & & Finance & \\
\hline 26 & Executive & & Corporate & \\
\hline 27 & Executiwe & & Finance & \\
\hline 28 & Executiwe & & Finance & \\
\hline 29 & Executive & & Finance & \\
\hline 30 & Executive & CFO & Finance & \\
\hline 31 & Executive & & Corporate & \\
\hline 32 & Executive & & Corporate & \\
\hline 33 & Executive & & Finance & \\
\hline 34 & $\mathrm{VP}$ & & Finance & \\
\hline 35 & $\mathrm{VP}$ & & Corporate & \\
\hline 36 & $\mathrm{VP}$ & & Finance & \\
\hline 37 & Other & Assist VP & Other & Constructionoperations \\
\hline 30 & Executive & & Finance & \\
\hline 39 & Executive & & Finance & \\
\hline 40 & Executive & & Corporate & \\
\hline 41 & Executive & & Finance & \\
\hline 42 & Executive & & Finance & \\
\hline 43 & Executive & & Finance & \\
\hline 44 & Executive & & Finance & \\
\hline 45 & Executiwe & & Finance & \\
\hline 46 & Other & controller & Finance & \\
\hline 47 & Executiwe & & Corporate & \\
\hline 48 & Executive & & Finance & \\
\hline
\end{tabular}




\begin{tabular}{|c|c|c|c|c|}
\hline $\begin{array}{l}\text { Fesp. } \\
\text { ID }\end{array}$ & positiorn & & department & \\
\hline 49 & Executive & & Finarice & \\
\hline 50 & Executive & & & \\
\hline 51 & Executive & & Finarice & \\
\hline 52 & Executive & & Finarice & \\
\hline 53 & $\mathrm{~V} P$ & & Finaroe & \\
\hline 54 & $\mathrm{VP}$ & & Fingrice & \\
\hline 55 & Other & Snr Dir F in. Head of IF & Finarice & \\
\hline 56 & Executive & & $\mathrm{HR}$ & \\
\hline 57 & $\mathrm{VP}$ & & Corporate & \\
\hline 58 & Executive & & Finarice & \\
\hline 59 & Execoutive & & Finance & \\
\hline 60 & Execoutive & & Corporate & \\
\hline 61 & Execoutive & & Finance & \\
\hline 62 & $\mathrm{VP}$ & & Finarice & \\
\hline 63 & Executive & & Corporate & \\
\hline 64 & Executive & & Corporate & \\
\hline 65 & Execoutive & & Corporate & \\
\hline 66 & Executive & & Finarice & \\
\hline 67 & Other & $\mathrm{gE}$ & Productiori & \\
\hline 68 & Executive & & Finatios & \\
\hline 69 & Execoutive & & Finance & \\
\hline 70 & Executive & & Corporate & \\
\hline 71 & Other & Dir \& Ir'vestor Relations & Finarice & \\
\hline 72 & Other & Acoountant & Finarice & \\
\hline 73 & Executive & & Other & CF O - oversee fin 8 operations \\
\hline 74 & Executive & & Finance & \\
\hline 75 & Other & Controller & Finance & \\
\hline 76 & Executive & & Finarice & \\
\hline
\end{tabular}




\begin{tabular}{|c|c|c|c|c|}
\hline $\begin{array}{l}\text { Resp. } \\
\text { ID }\end{array}$ & $\begin{array}{l}\text { Number of } \\
\text { Finaricial } \\
\text { Ml easures }\end{array}$ & $\begin{array}{l}\text { Wumber of } \\
\text { Internal } \\
\text { Operating } \\
\text { Measures }\end{array}$ & $\begin{array}{l}\text { Number of } \\
\text { Employee } \\
\text { Measures }\end{array}$ & $\begin{array}{l}\text { Wumber of } \\
\text { Customer } \\
\text { Weasures }\end{array}$ \\
\hline 1 & 0 & 0 & D & ] \\
\hline 2 & 10 & 5 & 5 & 3 \\
\hline 3 & 4 & 7 & 2 & 5 \\
\hline 4 & 50 & 20 & 15 & 20 \\
\hline 5 & 20 & 30 & 5 & 25 \\
\hline 6 & 10 & 12 & 6 & 10 \\
\hline 7 & 4 & 20 & 2 & 20 \\
\hline 8 & 5 & 20 & 5 & 5 \\
\hline 9 & 20 & 20 & 5 & 10 \\
\hline 10 & 6 & 8 & 10 & 8 \\
\hline 11 & 20 & 30 & 15 & 20 \\
\hline 12 & 20 & 100 & 20 & 50 \\
\hline 13 & 10 & 3 & 4 & 3 \\
\hline 14 & 10 & 10 & 5 & 8 \\
\hline 15 & 14 & 15 & 0 & 3 \\
\hline 16 & 9 & 8 & 4 & 3 \\
\hline 17 & & 1 & 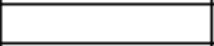 & \\
\hline 18 & 3 & 3 & 2 & 4 \\
\hline 19 & 10 & 5 & 3 & 8 \\
\hline 20 & 10 & 2 & 5 & 7 \\
\hline 21 & 8 & 2 & 3 & 4 \\
\hline 22 & 7 & 2 & 5 & 4 \\
\hline 23 & 10 & 10 & $T$ & 4 \\
\hline 24 & 300 & 400 & 50 & 100 \\
\hline 25 & 6 & 5 & 0 & 3 \\
\hline 26 & 7 & 10 & 0 & 3 \\
\hline 27 & 20 & 10 & 4 & 6 \\
\hline 29 & 10 & 10 & & \\
\hline 29 & 8 & 5 & 3 & 2 \\
\hline 30 & 50 & 100 & 25 & 25 \\
\hline 31 & 10 & 20 & 10 & 10 \\
\hline 32 & 10 & 15 & 5 & 8 \\
\hline 33 & 7 & 3 & 2 & 2 \\
\hline 34 & 5 & 5 & 1 & 1 \\
\hline 35 & 8 & 5 & 5 & 4 \\
\hline 36 & 5 & 15 & 2 & 3 \\
\hline 37 & 9 & 12 & 4 & 7 \\
\hline 39 & 4 & 5 & 3 & 2 \\
\hline 39 & 40 & 10 & 10 & 10 \\
\hline 40 & 13 & 200 & 10 & 20 \\
\hline 41 & 20 & 25 & 6 & 10 \\
\hline 42 & 7 & 3 & 0 & 2 \\
\hline 43 & 10 & 20 & 5 & 10 \\
\hline 44 & 13 & 8 & 4 & 1 \\
\hline 45 & 25 & 15 & 15 & 5 \\
\hline 46 & 2 & 4 & 2 & 2 \\
\hline 47 & 10 & 4 & 2 & 1 \\
\hline 48 & 20 & 20 & 5 & 5 \\
\hline
\end{tabular}




\begin{tabular}{|c|c|c|c|c|}
\hline $\begin{array}{l}\text { Resp. } \\
\text { ID }\end{array}$ & $\begin{array}{l}\text { Number of } \\
\text { Financial } \\
\text { hol easures }\end{array}$ & $\begin{array}{l}\text { Number of } \\
\text { Internal } \\
\text { operating } \\
\text { weasures }\end{array}$ & $\begin{array}{l}\text { Wumber of } \\
\text { Employee } \\
\text { W easures }\end{array}$ & $\begin{array}{l}\text { pumber of } \\
\text { Custorner } \\
\text { hl essures }\end{array}$ \\
\hline 49 & 15 & 10 & 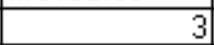 & - \\
\hline 50 & & & & \\
\hline 51 & 5 & 0 & 0 & 0 \\
\hline 52 & 12 & 24 & 10 & 10 \\
\hline 53 & 15 & 10 & 2 & 8 \\
\hline 54 & 6 & 1 & 1 & 4 \\
\hline 55 & 10 & 5 & 4 & 5 \\
\hline 56 & 5 & 5 & 7 & 6 \\
\hline 57 & 35 & 0 & 0 & 10 \\
\hline 59 & 4 & 30 & 2 & \\
\hline 59 & 25 & 50 & 10 & 30 \\
\hline 60 & 10 & 5 & 5 & 1 \\
\hline 61 & 12 & 15 & 24 & 12 \\
\hline 62 & 6 & 7 & 25 & 5 \\
\hline 63 & 12 & 12 & 6 & 10 \\
\hline 64 & 5 & 25 & 5 & 10 \\
\hline 65 & 10 & 6 & 10 & 3 \\
\hline 66 & 7 & 5 & 4 & 2 \\
\hline 67 & 4 & 6 & 1 & 4 \\
\hline 68 & 15 & 10 & 5 & 5 \\
\hline 69 & 25 & 30 & 4 & 10 \\
\hline 70 & 7 & 10 & 3 & 2 \\
\hline 71 & 15 & 20 & 5 & 10 \\
\hline 72 & 5 & 0 & 0 & 0 \\
\hline 73 & 20 & 40 & 15 & 15 \\
\hline 74 & 20 & 20 & 10 & 10 \\
\hline 75 & 10 & 1 & 2 & 0 \\
\hline 76 & 20 & 50 & 20 & 10 \\
\hline
\end{tabular}




\begin{tabular}{|c|c|c|c|c|}
\hline & Fank 1 & Fank 2 & Fank 3 & Fank 4 \\
\hline \multicolumn{5}{|l|}{1} \\
\hline 2 & Identity Processes & Eackground Processes & Whandated Processes & Friority Processes \\
\hline 3 & Priarity Processes & Eackgrourod Processes & I dertity' Processes & Wandated Frocesses \\
\hline 4 & Identity Processes & Friority Processes & Gackground Frocesses & Wandated Frocesses \\
\hline 5 & Wandated Frocesses & Background Frocesses & I dertity Processes & Friority Processes \\
\hline 6 & Friority Processes & Background Frocesses & I dertity Processes & Wandated Frocesses \\
\hline 7 & Identity Processes & Eackground Processes & Priority Processes & Mandated Processes \\
\hline 8 & Priarity Proceses & Mandated Processes & Eackground Processes & Identity' Processes \\
\hline 9 & Identity Processes & Priority Processes & Eackground Processes & Mandated Frocesses \\
\hline 10 & Priarity Processes & Whandated Frocesses & Eackground Frocesses & Idertity Processes \\
\hline 11 & Background Frocesses & Wandated Frocesses & Friority Processes & Idertity Processes \\
\hline 12 & Identity Processes & Friority Processes & Eackground Processes & Wandated Frocesses \\
\hline 13 & Background Froceses & Friority Processes & Whanded Processes & Identity Processes \\
\hline 14 & Background Froceses & Wandated Frocesses & Friority Frocesses & Idertity Processes \\
\hline 15 & Background Frocesses & Friority Processes & I dentity Processes & Wandated Frocesses \\
\hline 16 & Background Processes & W andated Processes & Identity Processes & Priarity Processes \\
\hline 17 & Mandated Processes & Background Processes & I dentity Processes & Friority Processes \\
\hline 18 & Background Frocesses & W andated Processes & Priority Processes & Identity Processes \\
\hline 19 & Identity Processes & Backgroulod Frocesses & Friority Processes & Warodated Processes \\
\hline 20 & Wandated Frocesses & Backgraund Frocesses & Friority Processes & Identity Processes \\
\hline 21 & Wandated Frocesses & Identity Frocesses & Friority Processes & Background Frocesses \\
\hline 22 & W andated Processes & Identity Processes & Friority Processes & Background Processes \\
\hline 23 & Identity Processes & Friority Processes & Whandated Frocesses & Background Processes \\
\hline 24 & Mandated Frocesses & Friority Processes & | dertity Proceses & Background Processes \\
\hline 25 & Priarity Processes & Eackgraurod Frocesses & Wardated Processes & Identity' Processes \\
\hline 26 & Background Frocesses & Friority Processes & I dentity Processes & Wandated Frocesses \\
\hline 27 & Background Processes & Priority Processes & I dentity Processes & Mandated Frocesses \\
\hline \multicolumn{5}{|l|}{29} \\
\hline 29 & Background Froceses & Identity Processes & Friority Frocesses & handated Frocesses \\
\hline 30 & Identity Frocesses & Maridated Processes & Friority Processes & Background Processes \\
\hline 31 & Priority Processes & Eackground Processes & I dentity Processes & Mandated Processes \\
\hline 32 & Background Proceses & Wandated Frocesses & Identity Processes & Priority Processes \\
\hline 33 & Identity Processes & Backgrourud Processes & Whanded Processes & Friority Processes \\
\hline 34 & Priarity' Processes & W ahodated Frocesses & Eackground Frocesses & Identity Processes \\
\hline 35 & Priarity Processes & Identity Processes & Background Processes & Mandated Frocesses \\
\hline 36 & Priarity Processes & Ideritity Processes & Whatated Processes & Background Processes \\
\hline 37 & Background Procesos & M andated Processes & Priority Processes & Identity Processes \\
\hline 38 & Wandated Frocesses & Friority Processes & I dentity Frocesses & Background Processes \\
\hline 39 & Identity Frocesses & Wandated Frocesses & Friority Processes & Background Frocesses \\
\hline 40 & Priarity Processes & W andated Processes & Eackground Processes & Identity Processes \\
\hline 41 & Priarity Processes & M andated Processes & Eackground Processes & Identity' Processes \\
\hline 42 & Priarity Processes & Wh andated Processes & Eackground Processes & Idertity Processes \\
\hline 43 & P'riarity' Processes & Ideritity Processes & Gackground Processes & Wahodated Frocesses \\
\hline 44 & Wandated Frocesses & Friority Processes & I dertity Processes & Background Frocesses \\
\hline 45 & Background Frocesses & Identity Frocesses & Whanded Frocesses & Friority Processes \\
\hline 46 & Background Proceses & Wandated Processes & Priority Processes & Idertity' Processes \\
\hline 47 & Priarity Processes & Warndated Frocesses & Eackground Processes & Identity Processes \\
\hline 48 & Background Processes & Mandated Processes & Priority Processes & Identity Processes \\
\hline
\end{tabular}




\begin{tabular}{|c|c|c|c|c|}
\hline $\mathrm{ID}$ & Fank 1 & Fark 2 & Fank3 & Fank 4 \\
\hline 49 & W andated Processes & Background Frocesses & Friority Processes & Ionentity' Processes \\
\hline 50 & Background Froceseses & Wandated Processes & Priority Processes & Identity Processes \\
\hline 51 & Background Frocesses & Identity Processes & Friority Processes & Mandated Processes \\
\hline 52 & Background Frocesses & |rentity' Processes & Priority Processes & W andated Frocesses \\
\hline 53 & Whatated Frocesses & Background Froceseses & Friority Processes & Identity Frocesses \\
\hline 54 & M andated Processes & Identity Processes & Friority Processes & Background Processes \\
\hline 55 & Identity' Processes & Friority Processes & Eackgroun Processes & Wandated Frocesses \\
\hline 56 & Friarity Pron:esses & |dentity Processes & W andated Processes & Backgroulnd Processes \\
\hline 57 & W andated Processes & Friority Processes & Identity Processes & Background Processes \\
\hline 58 & Priority Processes & Background Frocesses & Identity Processes & Wandated Frocesses \\
\hline 59 & Whated Frocesses & Identity Processes & Eackground Frocesses & Priority Processes \\
\hline 60 & Background Frocesses & W andated Processes & Friority Processes & Identity' Processes \\
\hline 61 & Windated Processes & Background Processes & Priority Processes & Identity Processes \\
\hline 62 & Whated Frocesses & Background Froceses & I dentity Processes & Priority Processes \\
\hline 63 & Background Froceses & Friority Frocesses & I dentity Processes & Wandated Frocesses \\
\hline 64 & Background Processes & Wandated Processes & Identity Processes & Priarity Processes \\
\hline 65 & Background Processes & Wandated Processes & Friority Processes & Identity Processes \\
\hline 66 & Identity' Processes & Background Froceses & Friority Processes & Wandated Frocesses \\
\hline 67 & Priority Processes & Identity Processes & Wandated Proceseses & Background Processes \\
\hline 68 & Mandated Processes & Priority Processes & Background Processes & Identity Processes \\
\hline 60 & Priority Processes & Mandated Processes & Background Proceses & \\
\hline 70 & Background Processes & Wandated Processes & Identity Proceseses & Priarity Processes \\
\hline 71 & Background Processes & Identity Processes & Priority Processes & Mandated Processes \\
\hline 72 & & & & \\
\hline 73 & Background Processes & Wandated Frocesses & Friority Processes & Identity Processes \\
\hline 74 & Background Processes & W andated Processes & Priority Processes & Identity Processes \\
\hline 75 & Background Processes & Wandated Processes & & \\
\hline 76 & Irdentity Processes & Priority Processes & Wandated Processes & Background Processes \\
\hline
\end{tabular}




\begin{tabular}{|c|c|}
\hline $\begin{array}{l}\text { Resp. } \\
\text { ID }\end{array}$ & Comments or suggestions? \\
\hline 1 & $\begin{array}{l}\text { We are a very small biotechnology RQD compary that autsources most of our operational } \\
\text { tasks. Wost of your performance questions do not pertain to our current businese model. }\end{array}$ \\
\hline 12 & 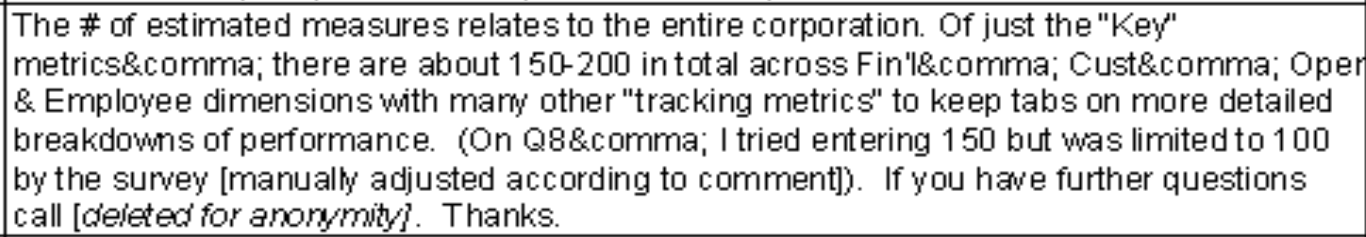 \\
\hline 15 & $\begin{array}{l}\text { With all the junk mail going around it took me a while to verify that this request was walid. I } \\
\text { found some phone numbers after I clicked on the link but clicking on links from unknown } \\
\text { sources in emails is not considered safe. }\end{array}$ \\
\hline 21 & $\begin{array}{l}\text { The responses are for our sole operating bank subsidiary. As a non-manufacturing } \\
\text { financial institution\&comma; most performance measurements are financial in nature and } \\
\text { standard for our industry. }\end{array}$ \\
\hline 24 & A good survey. Short and to the point. \\
\hline 30 & $\begin{array}{l}\text { Going formard you may want to consider using compliance with Sarbanes-oxley } \\
60 \times \text { gcomma; as many public companies now use their } 60 \times \text { testing program as a center } \\
\text { piece for monitoring performance. }\end{array}$ \\
\hline 46 & Confusing questions. \\
\hline 58 & Didn't really understand gestion 11 definitions. \\
\hline 73 & $\begin{array}{l}\text { Seems difficult to rank the processes indwidually as hopef uly the company's identity is } \\
\text { based on the premise of prowiding quality service daily gcomma; which is designed to meet } \\
\text { regulatory requirements. Therefore } 2 \text { comma; they all work together and by focusing on one } \\
\text { of the processesecomma; we are also addressing the other proceses at the same time. }\end{array}$ \\
\hline
\end{tabular}




\section{Appendix M: NAICS Response Categories Data and Dependent Variable Definition}

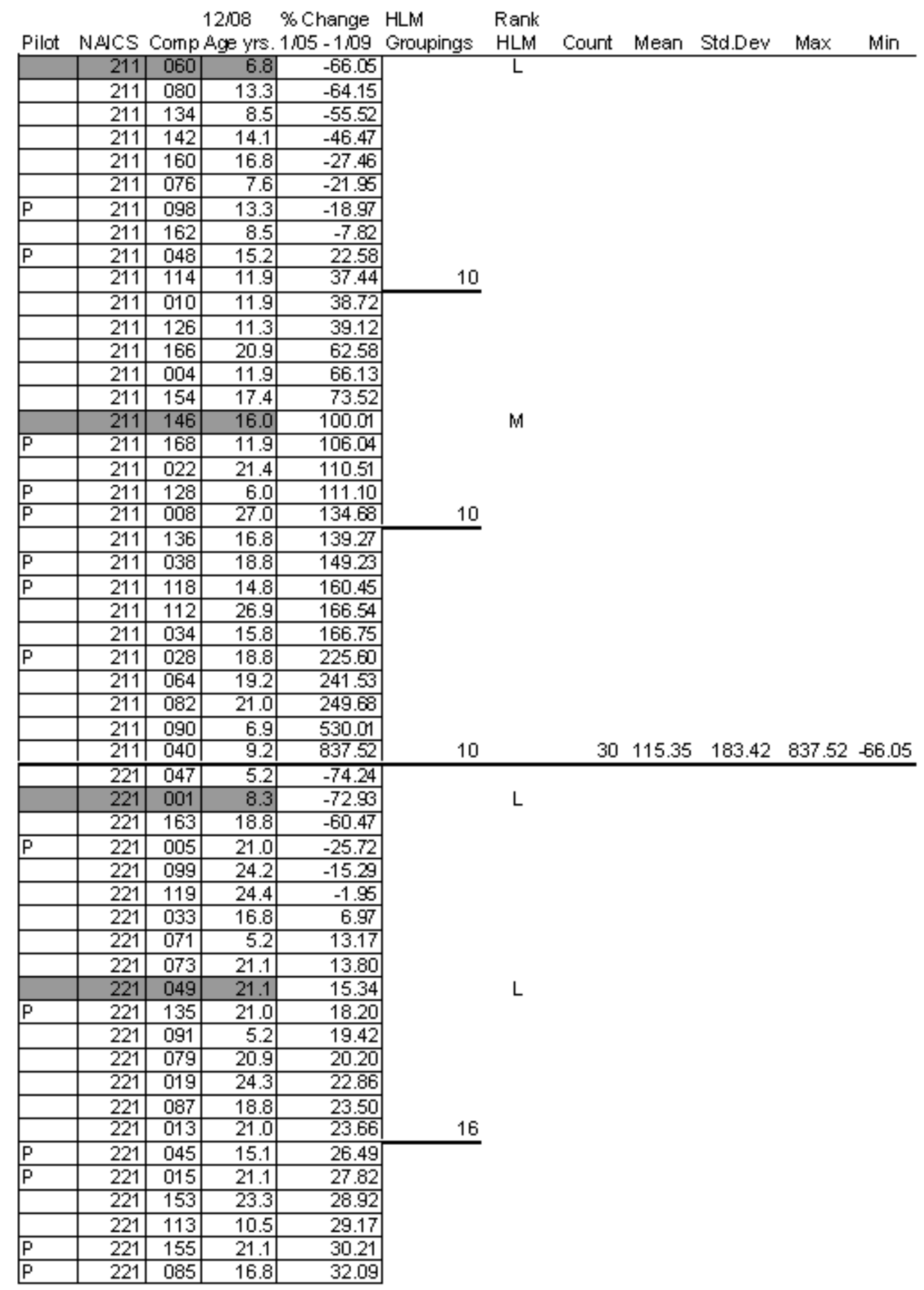


12,08 \% Change HLM Rank

Pilot NidCS CompAge yrs. 1,05-1,09 Groupings HLM Count Mean Std.Dev Max Min

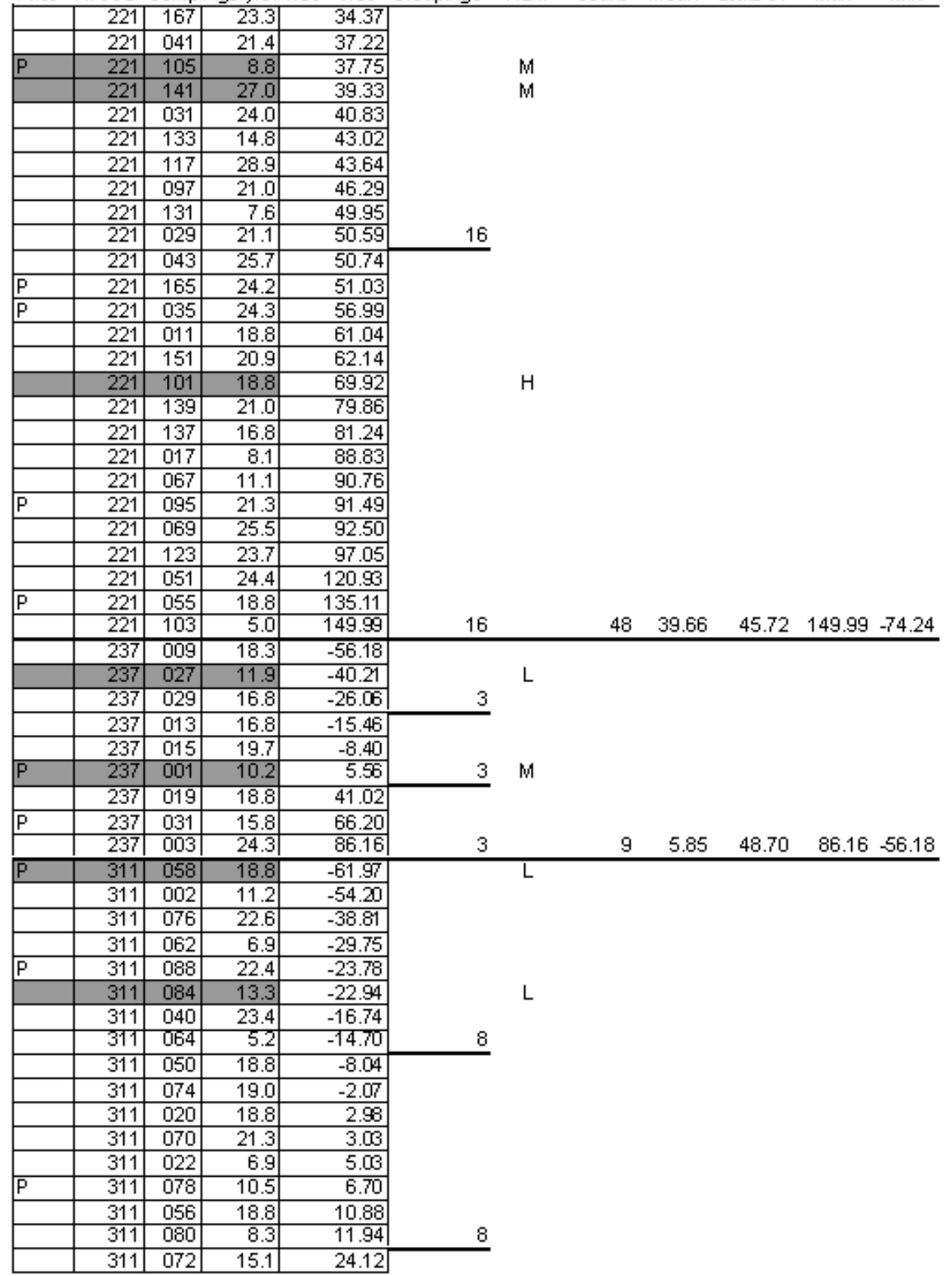


12,08 \% Change HLM Rank

Pilot NAJCS CompAge yrs. 1,05-109 Groupings HLM Count Mean Std.Dev Max Min

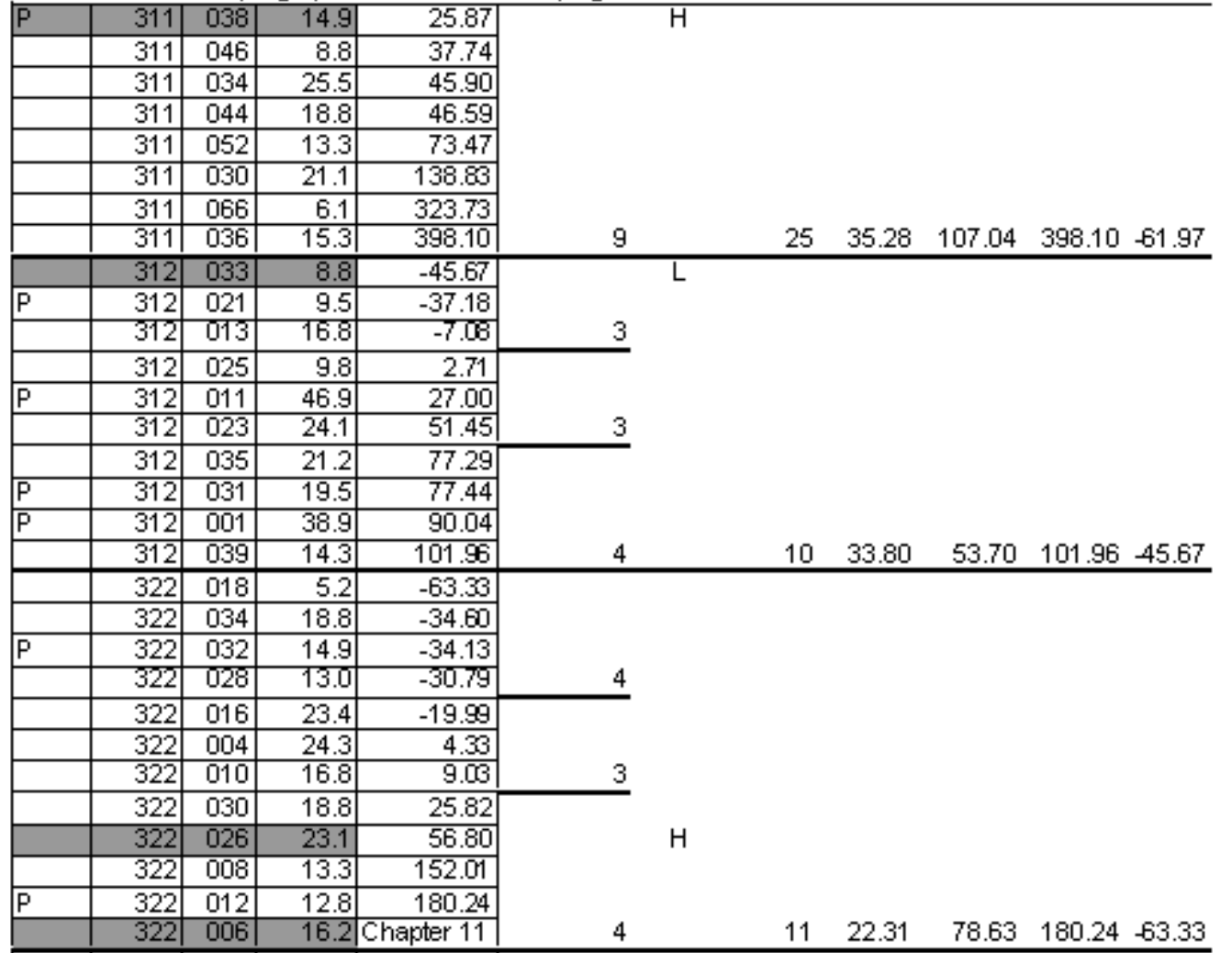

\begin{tabular}{|l|r|r|r|r|}
\hline$P$ & 325 & 113 & 11.8 & -98.73 \\
\hline & 325 & 539 & 8.5 & -98.10 \\
\hline & 325 & 541 & 13.3 & -97.42 \\
\hline & 325 & 361 & 8.7 & -96.90 \\
\hline & 325 & 407 & 18.8 & -96.65 \\
\hline & 325 & 511 & 14.6 & -96.16 \\
\hline & 325 & 419 & 10.5 & -95.69 \\
\hline & 325 & 365 & 8.5 & -95.27 \\
\hline$P$ & 325 & 513 & 8.3 & -94.46 \\
\hline & 325 & 291 & 8.4 & -93.55 \\
\hline & 325 & 069 & 15.3 & -91.63 \\
\hline & 325 & 001 & 21.3 & -89.15 \\
\hline & 325 & 427 & 13.2 & -88.51 \\
\hline & 325 & 017 & 12.5 & -86.91 \\
\hline & 325 & 245 & 11.3 & -86.66 \\
\hline & 325 & 181 & 16.8 & -86.26 \\
\hline$P$ & 325 & 053 & 8.8 & -81.76 \\
\hline$P$ & 325 & 273 & 12.2 & -81.34 \\
\hline$P$ & 325 & 423 & 10.3 & -80.64 \\
\hline & 325 & 241 & 12.4 & -78.93 \\
\hline & 325 & 435 & 18.8 & -76.88 \\
\hline & 325 & 399 & 17.1 & -76.48 \\
\hline
\end{tabular}


$12,08 \%$ Change HLM Rank

Pilot NACS CompAge yrs. 1,05-109 Groupings HLM Count Mean Std.Dev Max Min

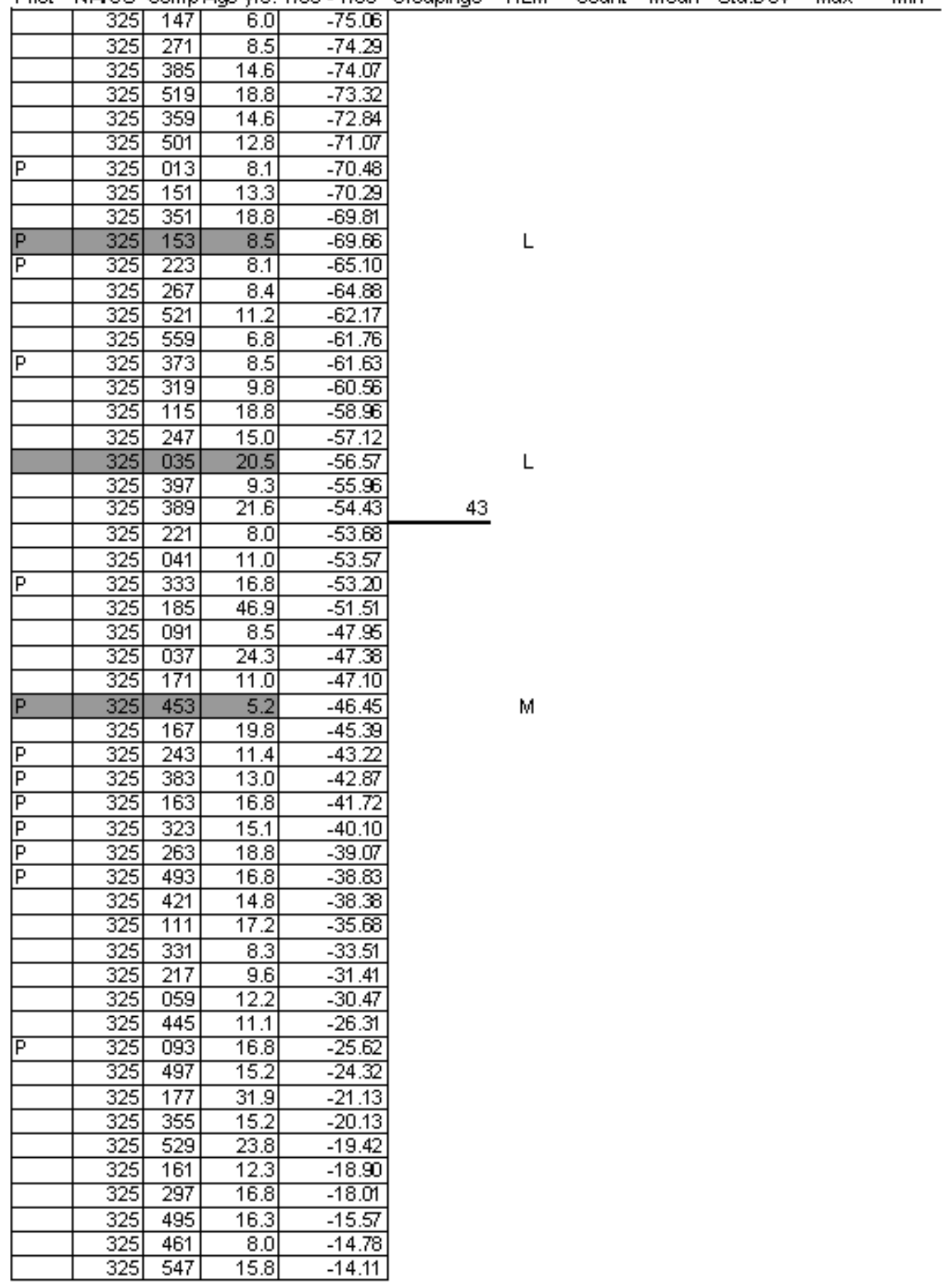


$12,08 \%$ Change HLM Rank

Pilot NACS CompAge yrs. 1,05-1,09 Groupings HLM Count Mean Std.Dev Max Min

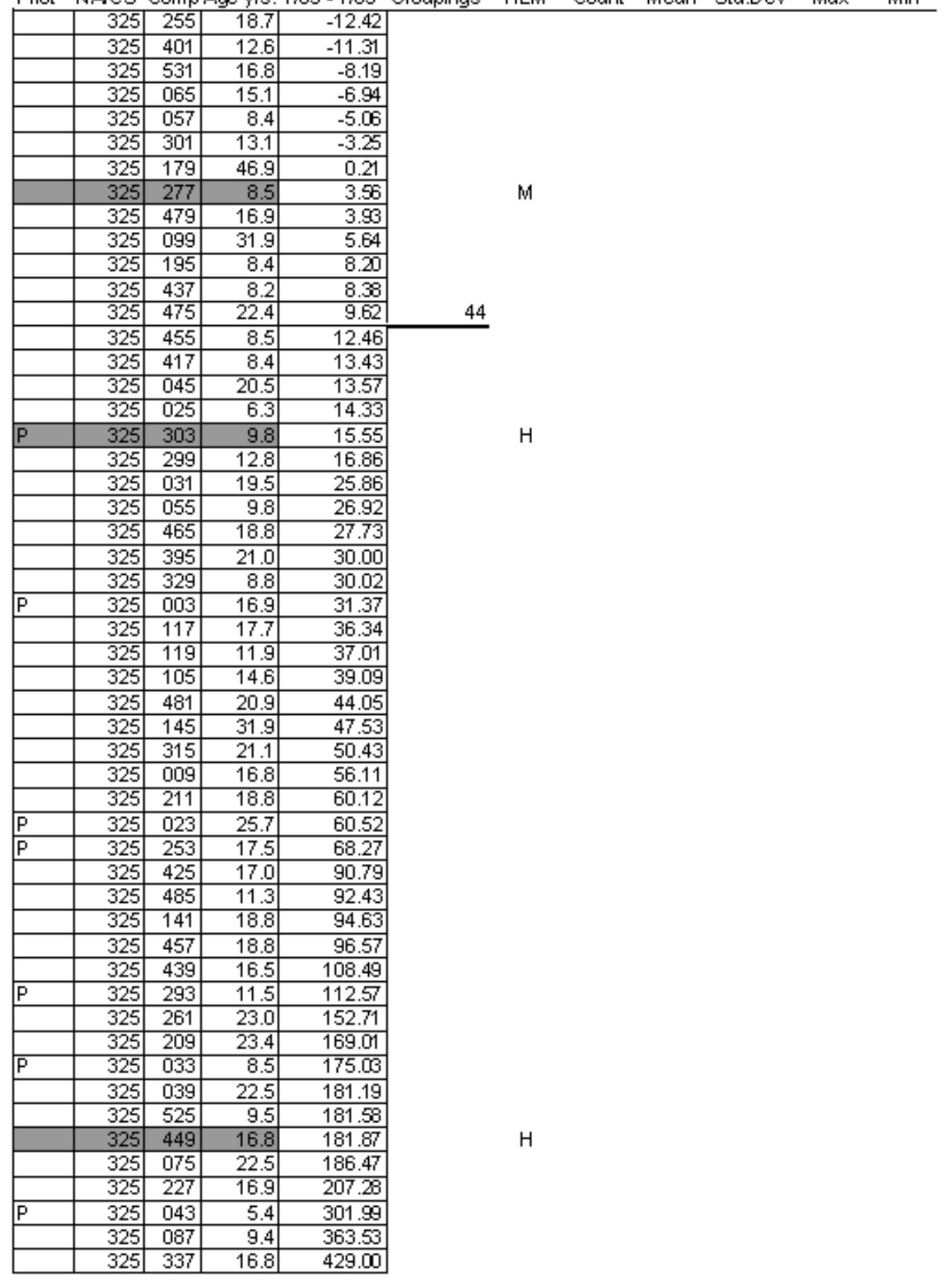


12,08 \% Change HLM Rank

Pilot NACS CompAge yrs. 1,05-1,09 Groupings HLM Count Mean Std.Dev Max Min

\begin{tabular}{|c|c|c|c|c|c|c|c|c|c|}
\hline $\mathrm{P}$ & 325 & 473 & 18.9 & 503.64 & \multirow[b]{5}{*}{44} & & \multirow[b]{5}{*}{131} & \multirow[b]{5}{*}{22.51} & \multirow[b]{5}{*}{$166.531007 .75 \quad-98.73$} \\
\hline & 325 & 447 & 12.1 & 582.35 & & & & & \\
\hline & 325 & 335 & 11.9 & 680.70 & & & & & \\
\hline & 325 & 107 & 5.2 & 727.67 & & & & & \\
\hline & 325 & 515 & 16.8 & 1007.75 & & & & & \\
\hline & 326 & $\overline{018}$ & 13.2 & -80.12 & & & & & \\
\hline $\mathrm{P}$ & 326 & 002 & 24.3 & -57.63 & & & & & \\
\hline & 326 & 010 & 21.1 & -49.44 & 3 & & & & \\
\hline & 326 & 034 & 11.9 & -37.05 & & & & & \\
\hline & 326 & 026 & 8.3 & -22.27 & & & & & \\
\hline$P$ & 326 & 022 & 16.8 & -4.07 & & & & & \\
\hline & 326 & 008 & 21.1 & -1.80 & 4 & & & & \\
\hline$P$ & 326 & 032 & 8.0 & 13.71 & & & & & \\
\hline & 326 & 004 & 15.7 & 80.18 & & & & & \\
\hline & 326 & 036 & 15.0 & 152.48 & 3 & $\mathrm{H}$ & 10 & -0.60 & $\begin{array}{lll}69.87 & 152.48 & -80.12\end{array}$ \\
\hline
\end{tabular}

\begin{tabular}{|l|l|l|r|r|}
\hline P & 332 & 015 & 8.5 & -67.46 \\
\hline
\end{tabular}

\begin{tabular}{l|r|r|r|r|}
\hline & 332 & 059 & 7.5 & -65.99 \\
\hline & 332 & 013 & 10.8 & -49.52 \\
\hline
\end{tabular}

\begin{tabular}{|c|c|c|c|c|}
\hline & 332 & 013 & 10.8 & -49.52 \\
\hline & 332 & 049 & 15.5 & -20.51 \\
\hline & 332 & 037 & 16.7 & -16.15 \\
\hline
\end{tabular}

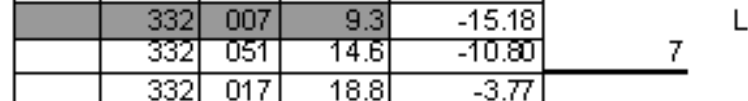

\begin{tabular}{l|r|r|r|r|}
\hline P & 332 & 065 & 18.8 & 3.53 \\
\hline
\end{tabular}

\begin{tabular}{|l|r|r|r|r|}
\hline$P$ & 332 & 005 & 24.3 & 20.93 \\
\hline$P$ & 332 & 055 & 21.0 & 25.38 \\
\hline
\end{tabular}

\begin{tabular}{|l|r|r|r|r|}
\hline$P$ & 332 & 055 & 21.0 & 25.38 \\
\hline & 332 & 001 & 24.3 & 29.91 \\
\hline
\end{tabular}

\begin{tabular}{l|r|r|r|r|}
\hline & 332 & 019 & 16.8 & 33.42 \\
\hline
\end{tabular}

\begin{tabular}{|l|l|l|l|l|l|}
\hline$P$ & 332 & 025 & 21.1 & 38.92 & 7 \\
\hline & 332 & 041 & 23.4 & 51.02 & \\
\hline
\end{tabular}

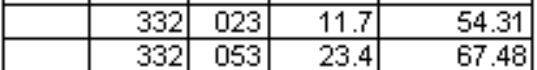

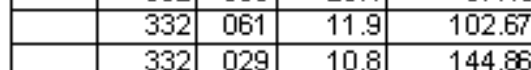

\begin{tabular}{|r|r|r|r|r|}
\hline & 332 & 029 & 10.8 & 144.86 \\
\hline & 332 & 047 & 21.9 & 194.39 \\
\hline
\end{tabular}

\begin{tabular}{|r|r|r|r|r|rrrrr}
\hline$P$ & 332 & 035 & 8.5 & 363.53 & & & & & \\
\hline & 332 & 039 & 16.8 & 400.51 & 8 & 58.25 & 122.35 & 400.51 & -67.46 \\
\hline
\end{tabular}

\begin{tabular}{|l|r|r|r|r|}
\hline $\mathrm{P}$ & 333 & 098 & 11.7 & -79.28 \\
\hline
\end{tabular}

\begin{tabular}{|c|c|c|c|c|}
\hline & 33 & & 62 & 18.8 \\
\hline & 33 & & 22 & 17.6 \\
\hline
\end{tabular}

\begin{tabular}{|l|l|l|l|l|}
\hline$P$ & 333 & 038 & 22.3 & -43.36 \\
\hline
\end{tabular}

\begin{tabular}{|l|l|l|l|l|}
\hline & 333 & 130 & 13.8 & -29.69 \\
\hline
\end{tabular}

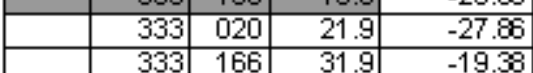

\begin{tabular}{|l|r|r|r|r|}
\hline $\mathrm{P}$ & 333 & 168 & 16.8 & -13.55 \\
\hline
\end{tabular}

\begin{tabular}{|l|r|r|r|r|}
\hline & 333 & 104 & 23.4 & -12.99 \\
\hline $\mathrm{P}$ & 333 & 148 & 5.2 & -5.90 \\
\hline
\end{tabular}

\begin{tabular}{|l|r|r|r|r|}
\hline & 333 & 006 & 15.8 & -1.10 \\
\hline & 333 & 030 & 8.5 & 1.99 \\
\hline$P$ & 333 & 118 & 16.3 & 4.16 \\
\hline$P$ & 333 & 078 & 21.1 & 5.40 \\
\hline & 333 & 066 & 21.8 & 12.20 \\
\hline
\end{tabular}


$12,08 \%$ Rank

Pilot NalCS CompAge yrs. 1,05-109 Groupings HLM Count Mean Std.Dev Max Min

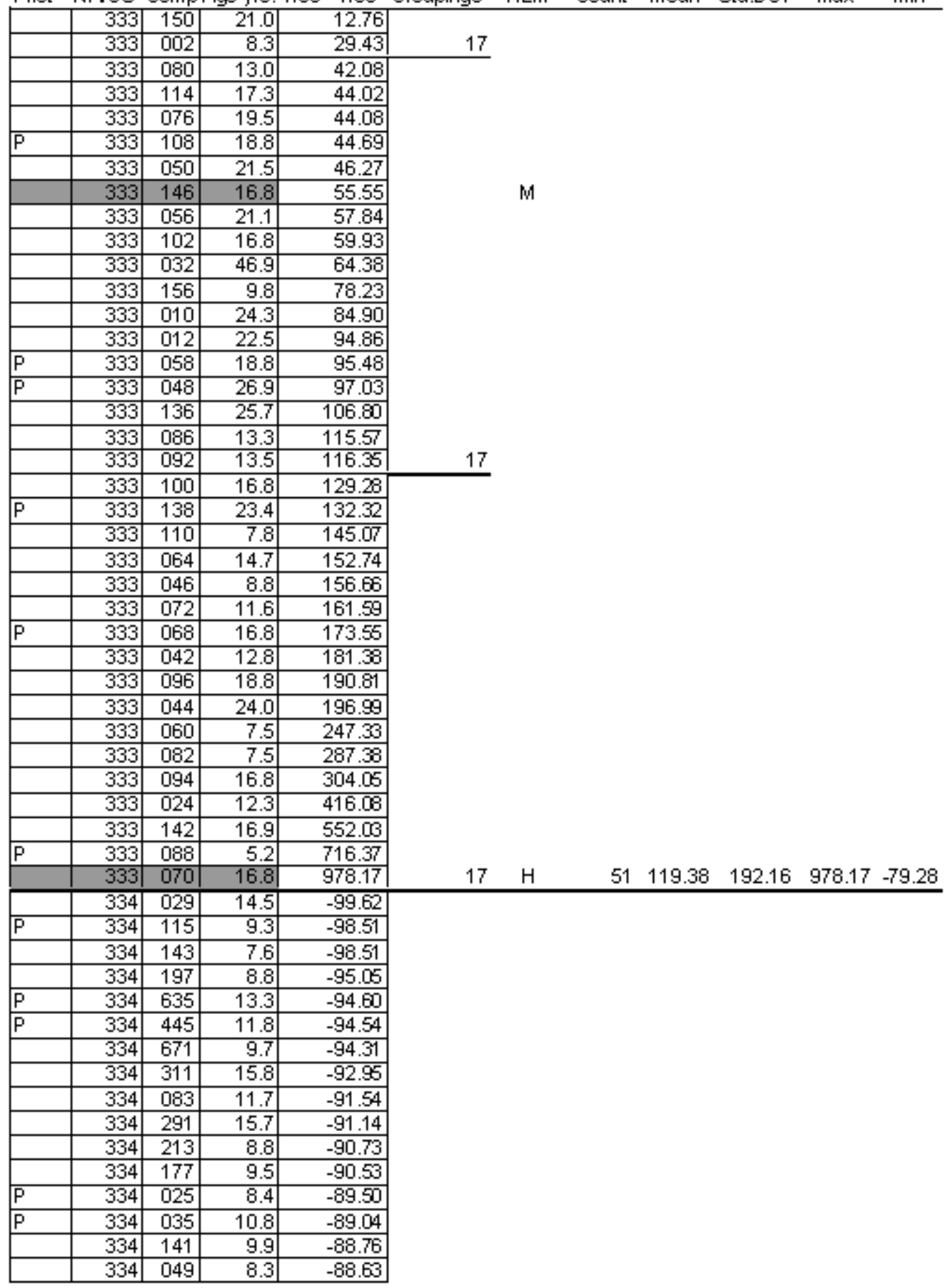


12,018 \% Change HLM Rank

Pilat NuCS Comp Age yrs 1,05-1,09 Groupings HLW Count Mean Std.Dev Max Min

\begin{tabular}{|c|c|c|c|c|}
\hline$\overline{\mathrm{P}}$ & 334 & 275 & 8.5 & -86.50 \\
\hline & 334 & 171 & 12.6 & -86.34 \\
\hline & 334 & 663 & 13.0 & -84.50 \\
\hline & 334 & ET7 & 5.1 & -84.17 \\
\hline & 334 & 447 & 8.4 & -83.53 \\
\hline & 334 & 289 & 16.8 & -83.34 \\
\hline \multirow[t]{5}{*}{$\bar{P}$} & 334 & 425 & 9.1 & -83.07 \\
\hline & 334 & 587 & 14.6 & -81.54 \\
\hline & 334 & 503 & 14.3 & -81.53 \\
\hline & 334 & 039 & 11.6 & -81.66 \\
\hline & 334 & 051 & 16.8 & -80.94 \\
\hline \multirow[t]{3}{*}{$P$} & 334 & 465 & 15.0 & -80.50 \\
\hline & 334 & 357 & 18.8 & -80.05 \\
\hline & 334 & 147 & 13.3 & -783 \\
\hline \multirow[t]{8}{*}{$P$} & 334 & 215 & 15.8 & -78.01 \\
\hline & 334 & 551 & 8.5 & -77.70 \\
\hline & 334 & 037 & 17.5 & -76.99 \\
\hline & 334 & 493 & 8.5 & $-7 B .41$ \\
\hline & 334 & 179 & 11.3 & -75.69 \\
\hline & 334 & 059 & 16.8 & -75.50 \\
\hline & 334 & 509 & 8.5 & -75.06 \\
\hline & 334 & 139 & 18.8 & -74.71 \\
\hline \multirow[t]{7}{*}{$\mathrm{P}$} & 334 & 135 & 12.6 & -74.53 \\
\hline & 334 & 543 & 9.9 & -74.38 \\
\hline & 334 & 201 & 14.0 & -72.66 \\
\hline & 334 & 597 & 14.9 & -71.15 \\
\hline & 334 & 661 & 16.8 & -70.85 \\
\hline & 334 & 463 & 18.8 & -69.60 \\
\hline & 334 & 359 & 7.1 & -6839 \\
\hline \multirow[t]{6}{*}{$\mathrm{P}$} & 334 & 385 & 12.3 & -68.69 \\
\hline & 334 & 541 & 13.1 & -67.12 \\
\hline & 334 & 111 & 6.8 & -66.40 \\
\hline & 334 & 321 & 15.1 & -66.32 \\
\hline & 334 & 127 & 18.8 & -65.93 \\
\hline & 334 & 087 & 13.0 & -65.75 \\
\hline \multirow[t]{11}{*}{$\bar{P}$} & 334 & 185 & 14.7 & -65.53 \\
\hline & 334 & 637 & 5.2 & -64.97 \\
\hline & 334 & 347 & 13.1 & -64.70 \\
\hline & 334 & 033 & 9.2 & -64.45 \\
\hline & 334 & 479 & 12.0 & -64.45 \\
\hline & 334 & 573 & 11.7 & -64.27 \\
\hline & 334 & 309 & 12.6 & -63.62 \\
\hline & 334 & 529 & 8.7 & -63.68 \\
\hline & 334 & 133 & 18.8 & -62.97 \\
\hline & 334 & 403 & 15.8 & -62.73 \\
\hline & 334 & 339 & 18.8 & -62.14 \\
\hline \multirow[t]{2}{*}{$\bar{P}$} & 334 & 015 & 5.2 & -61.46 \\
\hline & 334 & 161 & 16.8 & -61.41 \\
\hline $\bar{P}$ & 334 & 255 & 13.7 & -61.20 \\
\hline \multirow[t]{3}{*}{ P } & 334 & 615 & 13.5 & -61.08 \\
\hline & 334 & 613 & 8.4 & -60.77 \\
\hline & 334 & 667 & 13.6 & -60.13 \\
\hline
\end{tabular}


12,08 \% Change HLM Rank

Pilot NACS CompAge yrs. 1,05-1,09 Groupings HLM Count Mean Std.Dev Max Min

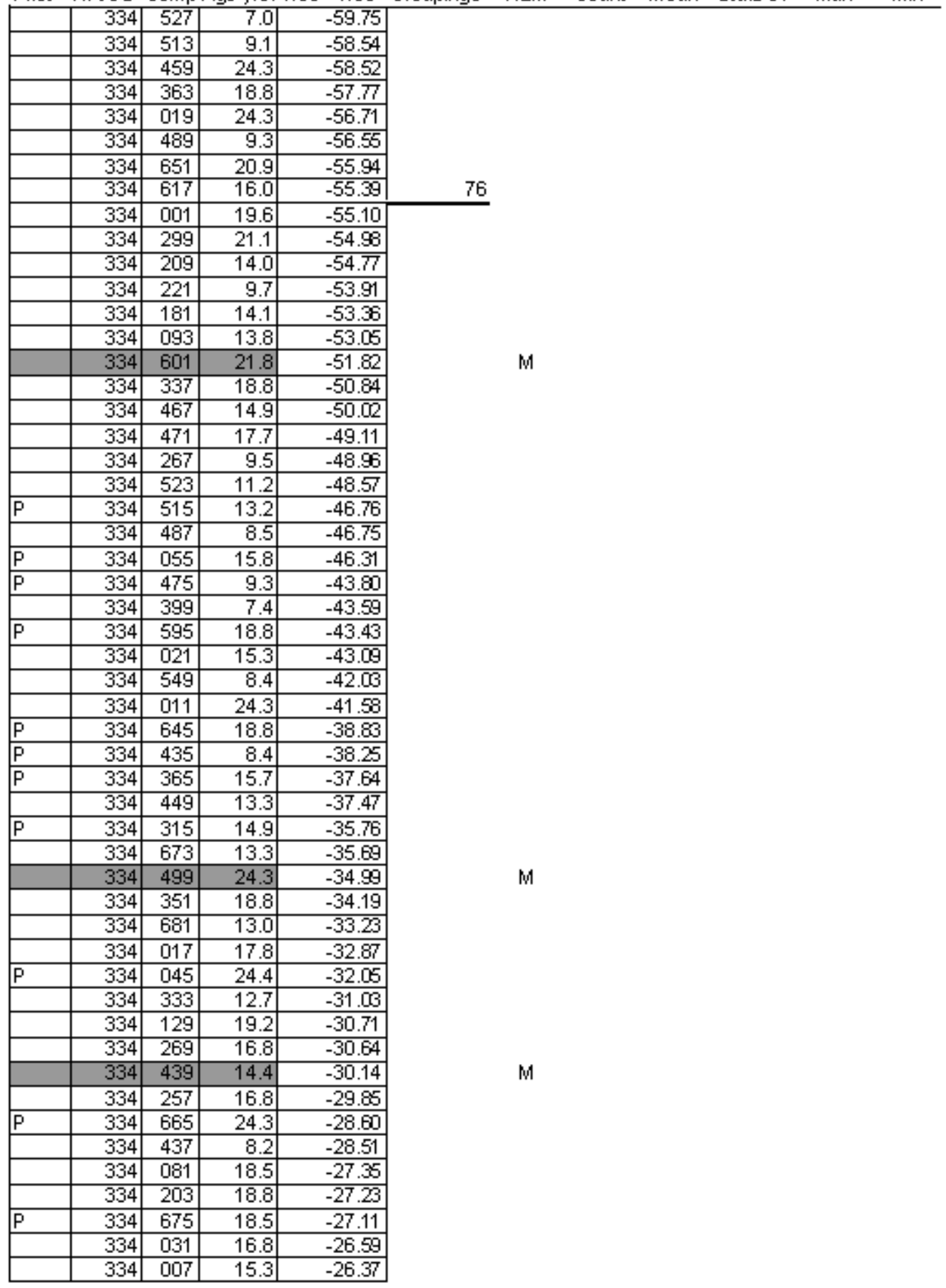


$12,08 \%$ Change HLM Rank

Pilot NAJCS CompAge yrs. 1,05-1,09 Groupings HLM Count Mean Std.Dev Max Min

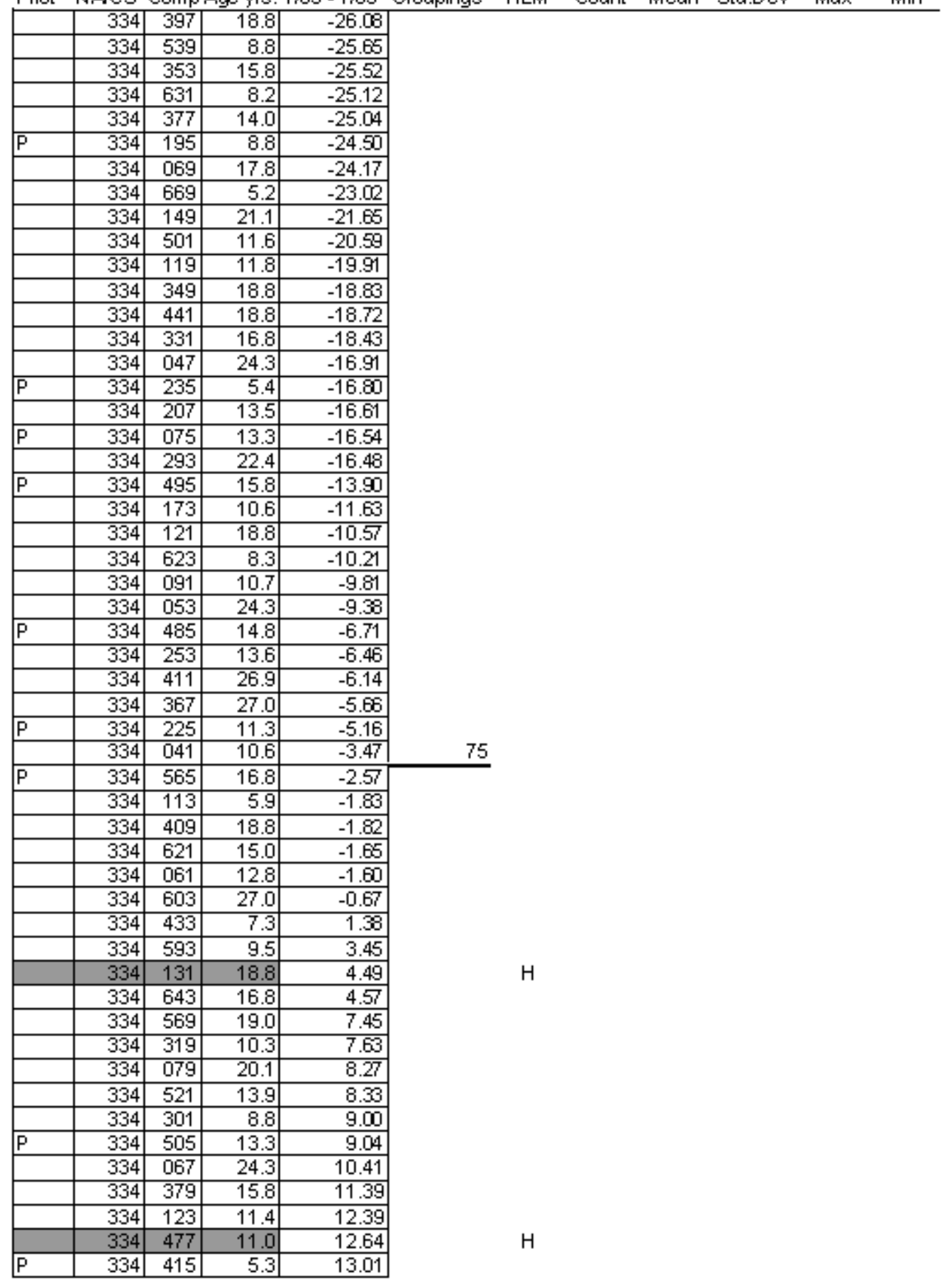


12,08 \% Change HLM Rank

Pilot NACS CompAge yrs. 1,05-1,09 Groupings HLM Count Mean Std.Dev Max Min

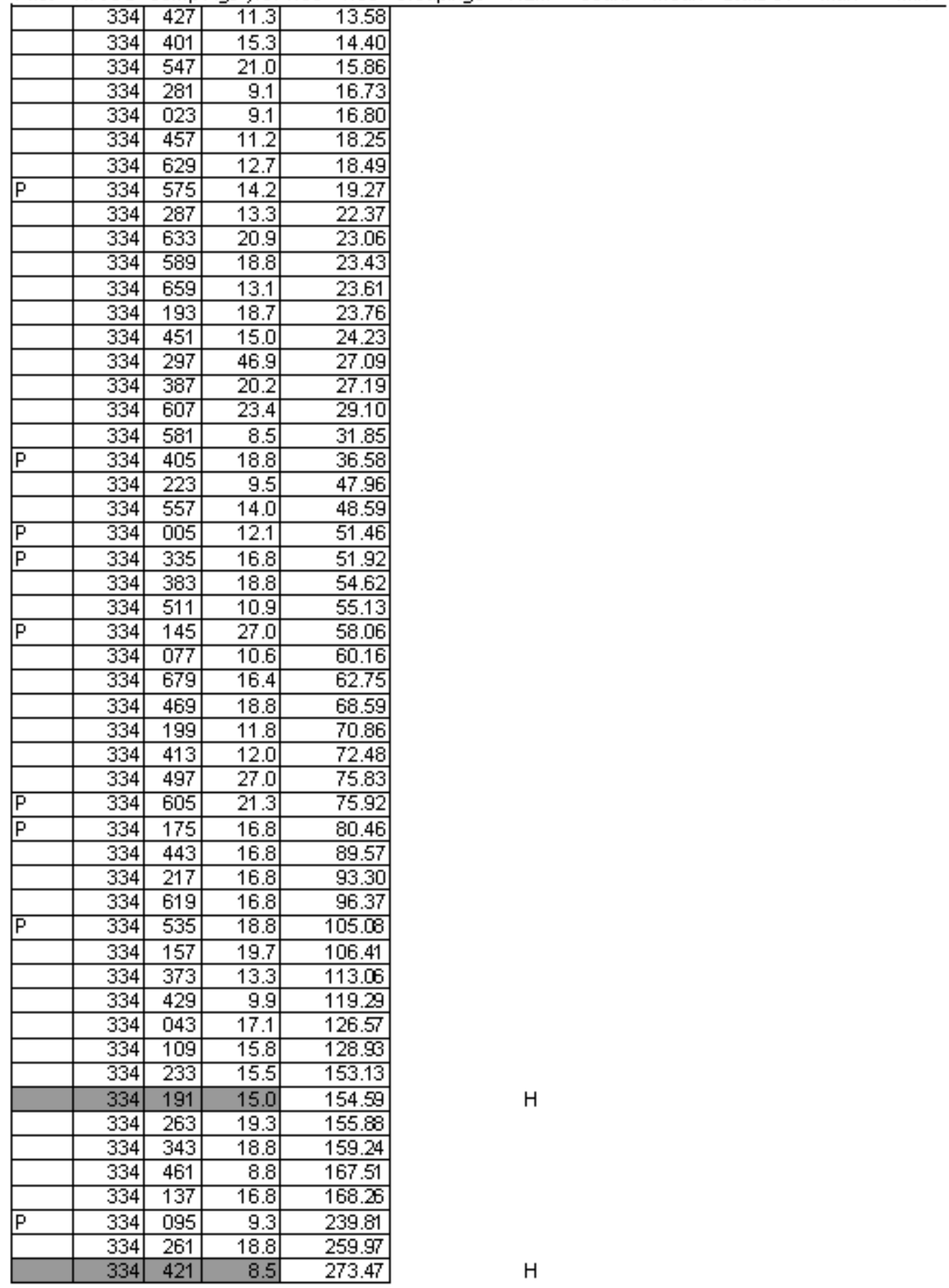


$12,08 \%$ Rank

Pilot NACS CompAge yrs. 1,05-1,09 Groupings HLM Count Mean Std.Dev Max Min

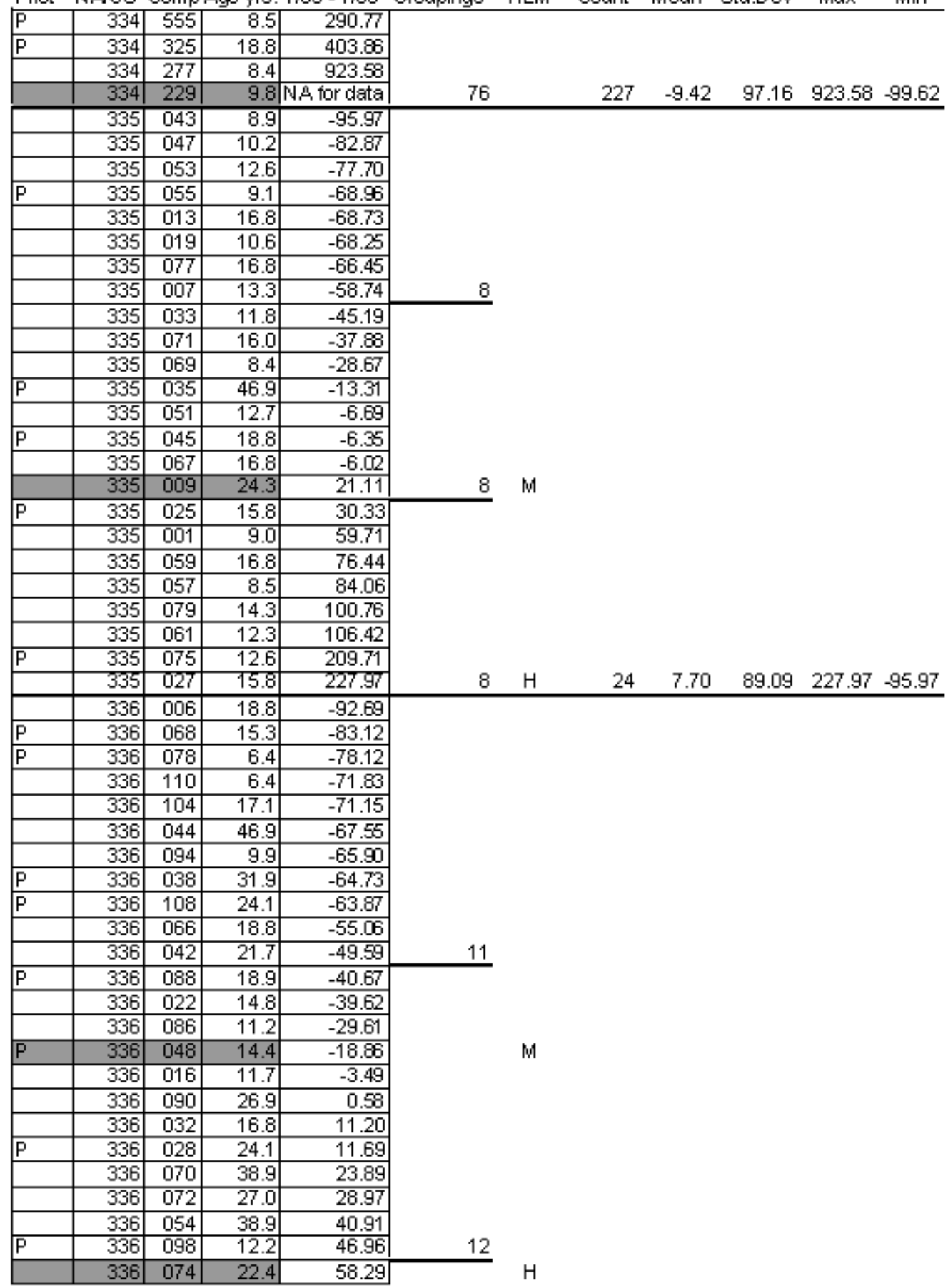


$12,08 \%$ Change HLM Rank

Pilot NACS Comp Age yrs. 1,05-1,09 Groupings HLM Count Mean Std.Dev Max Min

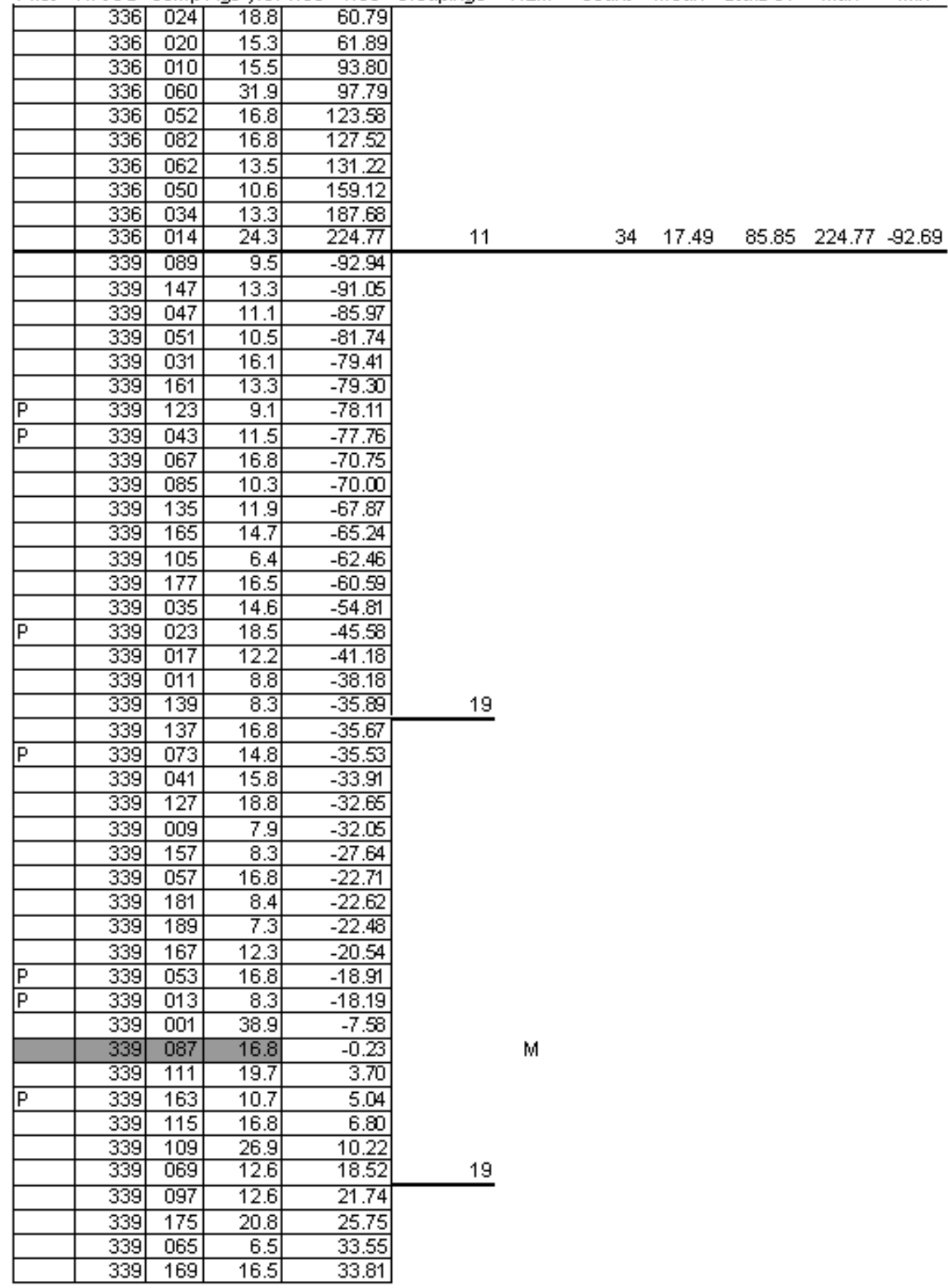


$12 \% 08$ \% Change HLM Rank

Pilot NAICS CompAge yrs. 1,05-1,09 Groupings HLM Count Mean Std.Dev Max Min

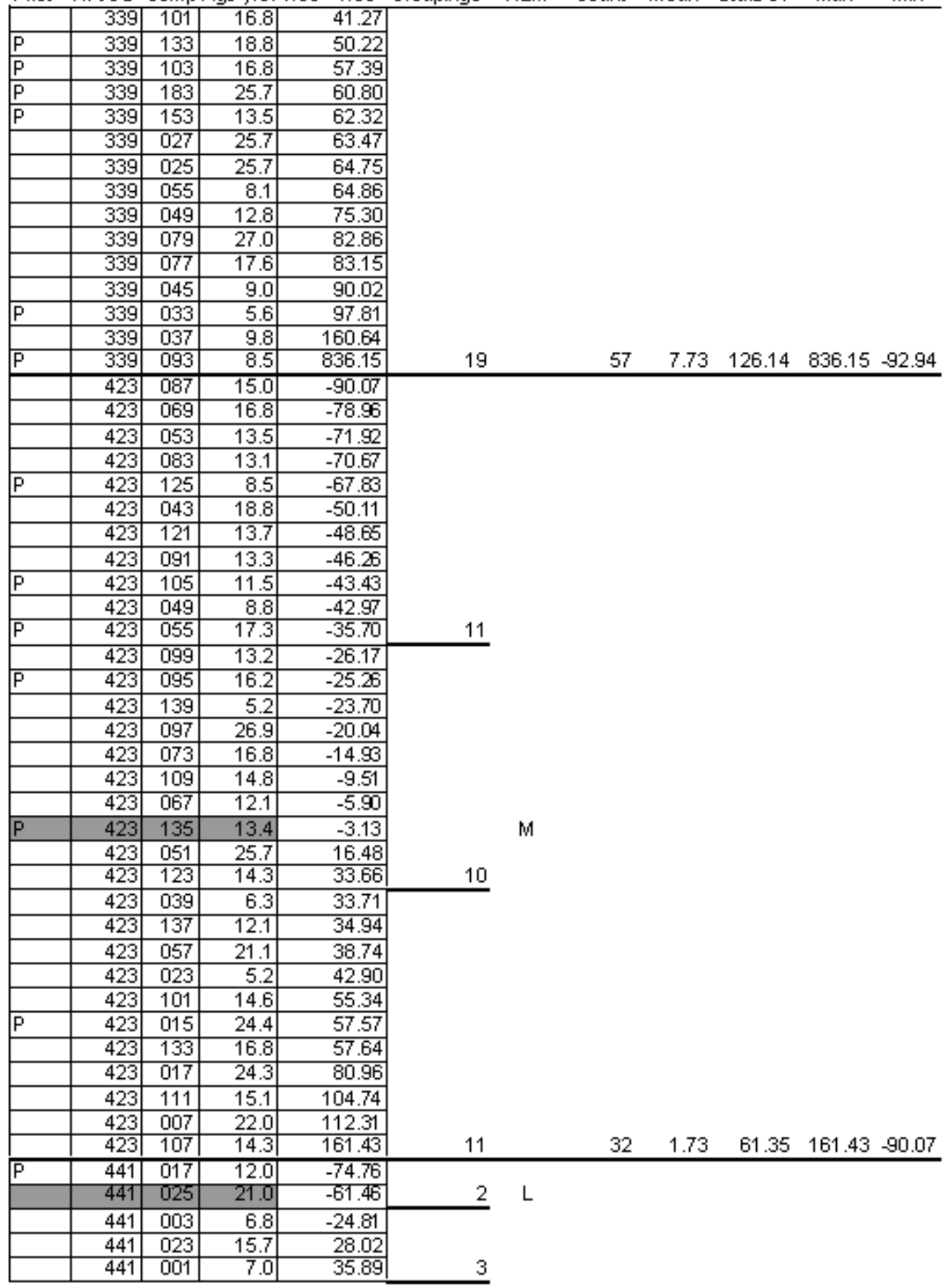


$12,08 \%$ Change HLM Rank

Pilot NACS CompAge yrs. 1,05-1,09 Groupings HLM Count Mean Std.Dev Max Min

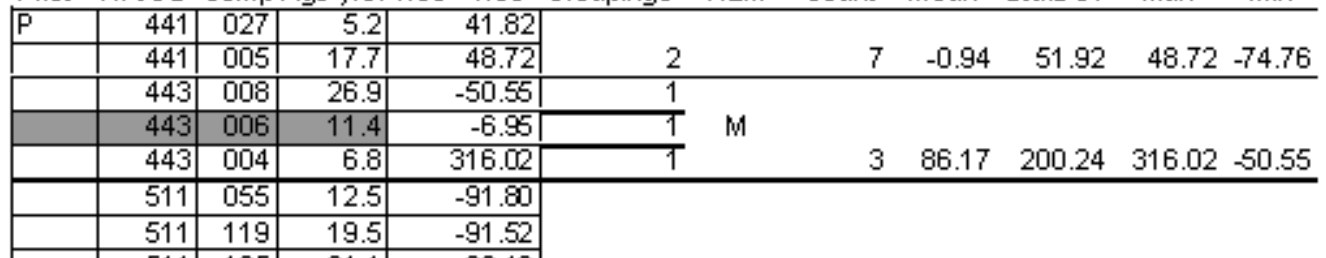

\begin{tabular}{|c|c|c|c|c|}
\hline & 511 & 105 & 21.1 & -89.18 \\
\hline & 511 & 211 & 9.9 & -87.14 \\
\hline & 511 & 111 & 9.8 & -84.71 \\
\hline
\end{tabular}

\begin{tabular}{rr|r|r|r|}
\hline & 511 & 035 & 12.5 & -78.01 \\
\hline & 511 & 157 & 13.1 & -76.30 \\
\hline
\end{tabular}

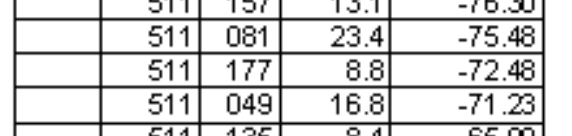

\begin{tabular}{|l|r|r|r|r|}
\hline & 511 & 135 & 8.4 & -65.90 \\
\hline & 511 & 117 & 9.2 & -61.14 \\
\hline$P$ & 511 & 093 & 11.6 & -56.86 \\
\hline
\end{tabular}

\begin{tabular}{|r|r|r|r|r|}
\hline$P$ & 511 & 093 & 11.6 & -56.06 \\
\hline & 511 & 021 & 13.4 & -48.69 \\
\hline & 511 & 071 & 9.6 & -44.97 \\
\hline$P$ & 511 & 123 & 23.4 & -44.30 \\
\hline$P$ & 511 & 037 & 18.8 & -44.01 \\
\hline$P$ & 511 & 013 & 11.4 & -40.02 \\
\hline
\end{tabular}

\begin{tabular}{|l|r|r|r|r|r|}
\hline$P$ & 511 & 013 & 11.4 & -40.02 & \multicolumn{1}{|c}{18} \\
\hline & 511 & 091 & 9.2 & -39.14 & \\
\hline & 511 & 011 & 24.3 & -37.59 \\
\hline & 511 & 167 & 10.5 & -35.59 \\
\hline & 511 & 147 & 9.1 & -34.17 \\
\hline & 511 & 067 & 18.8 & -28.43 \\
\hline & 511 & 139 & 20.3 & -28.24 \\
\hline & 511 & 151 & 10.6 & -25.27 \\
\hline & 511 & 159 & 8.2 & -23.97 & \\
\hline$P$ & 511 & 143 & 9.5 & -22.07 & \\
\hline
\end{tabular}

\begin{tabular}{|l|r|r|r|r|}
\hline$P$ & 511 & 143 & 9.5 & -22.07 \\
\hline & 511 & 175 & 16.8 & -21.27 \\
\hline & 511 & 001 & 18.1 & -18.20 \\
\hline & 511 & 115 & 10.7 & -17.03 \\
\hline & 511 & 019 & 9.5 & -14.22 \\
\hline
\end{tabular}

\begin{tabular}{|c|c|c|c|c|c|}
\hline & 511 & 019 & 9.5 & -14.22 & \\
\hline & 511 & 009 & 5.2 & -10.38 & \\
\hline & 511 & 145 & 8.3 & -10.05 & \\
\hline & 511 & 005 & 13.8 & -9.13 & \\
\hline & 511 & 031 & 9.8 & -6.26 & \\
\hline & 511 & 191 & 16.8 & -5.32 & 18 \\
\hline & 511 & 165 & 9.3 & 1.17 & \\
\hline & 511 & 199 & 16.8 & 1.99 & \\
\hline & 511 & 125 & 22.8 & 5.29 & \\
\hline & 511 & 065 & 10.4 & 16.16 & \\
\hline & 511 & 007 & 10.4 & 23.93 & \\
\hline$P$ & 511 & 023 & 23.4 & 25.73 & \\
\hline & 511 & 101 & 15.8 & 26.69 & \\
\hline & 511 & 195 & 8.3 & 27.02 & \\
\hline & 511 & 047 & 16.0 & 30.98 & \\
\hline & 511 & 149 & 12.3 & 31.19 & \\
\hline $\mathrm{P}$ & 511 & 223 & 18.8 & 33.88 & \\
\hline
\end{tabular}


12,08 \% Change HLM Rank

Pilot NASCS CompAge yrs. 1,05-1,09 Groupings HLM Count Mean Std.Dev Max Min

\begin{tabular}{|c|c|c|c|c|c|c|c|c|c|c|}
\hline & 511 & 041 & 13.0 & 36.33 & & & & & & \\
\hline & 511 & 189 & 17.3 & 65.31 & & & & & & \\
\hline $\mathrm{P}$ & 511 & 113 & 13.5 & 69.80 & & & & & & \\
\hline $\mathrm{P}$ & 511 & 213 & 12.1 & 78.47 & & & & & & \\
\hline & 511 & 051 & 12.6 & 92.96 & & & & & & \\
\hline & 511 & 207 & 10.5 & 106.38 & & & & & & \\
\hline & 511 & 097 & 9.3 & 115.21 & \multicolumn{2}{|l|}{18} & 54 & -15.21 & 51.83 & $115.21-91.80$ \\
\hline & 515 & 029 & 9.6 & -94.89 & \multirow[b]{4}{*}{4} & \multirow{10}{*}{$M$} & & & & \\
\hline & 515 & 037 & 9.2 & -92.02 & & & & & & \\
\hline $\mathrm{P}$ & 515 & 013 & 9.9 & -82.21 & & & & & & \\
\hline & 515 & 011 & 6.7 & -77.60 & & & & & & \\
\hline & 515 & 031 & 15.2 & -74.16 & \multirow[b]{3}{*}{3} & & & & & \\
\hline $\mathrm{P}$ & 515 & 023 & 6.6 & -72.47 & & & & & & \\
\hline & 515 & 009 & 8.5 & -51.58 & & & & & & \\
\hline & 515 & 027 & 13.3 & -49.98 & \multirow[b]{4}{*}{4} & & & & & \\
\hline & 515 & 015 & 12.6 & -34.33 & & & & & & \\
\hline & 515 & 035 & 13.5 & -22.13 & & \multirow{2}{*}{\multicolumn{2}{|c|}{11}} & & \multirow[b]{2}{*}{39.56} & \\
\hline & 515 & 025 & 15.8 & 41.03 & & & & -55.49 & & $41.03 \quad-94.89$ \\
\hline
\end{tabular}

\begin{tabular}{|l|r|r|r|r|}
\hline $\mathrm{P}$ & 522 & 531 & 8.5 & -89.00 \\
\hline
\end{tabular}

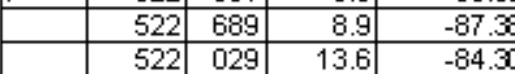

\begin{tabular}{|r|r|r|r|}
\hline 522 & 303 & 12.0 & -83.62 \\
\hline
\end{tabular}

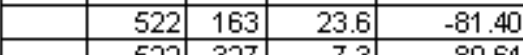

\begin{tabular}{r|r|r|r|r|} 
& 522 & 327 & 7.3 & -80.64 \\
\hline & 522 & 335 & 20.0 & -80.49 \\
\hline
\end{tabular}

\begin{tabular}{|l|r|r|r|r|}
\hline & 522 & 247 & 31.9 & -80.06 \\
\hline $\mathrm{P}$ & 522 & 081 & 8.5 & -79.70 \\
\hline
\end{tabular}

\begin{tabular}{r|r|r|r|r|}
\hline & 522 & 225 & 12.3 & -77.64 \\
\hline & 522 & 015 & 7.0 & -74.06 \\
\hline
\end{tabular}

\begin{tabular}{|r|r|r|r|r|}
\hline & 522 & 297 & 18.8 & -70.53 \\
\hline $\mathrm{P}$ & 522 & 261 & 18.8 & -67.93 \\
\hline
\end{tabular}

\begin{tabular}{|l|r|r|r|r|}
\hline $\mathrm{P}$ & 522 & 261 & 18.8 & -67.93 \\
\hline $\mathrm{P}$ & 522 & 131 & 13.4 & -67.66 \\
\hline
\end{tabular}

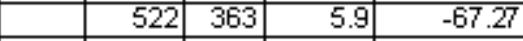

\begin{tabular}{r|r|r|r|r|}
\hline & 522 & 019 & 22.5 & -67.12 \\
\hline & 522 & 089 & 14.8 & -65.29 \\
\hline & 522 & 439 & 8.9 & -64.69 \\
\hline
\end{tabular}

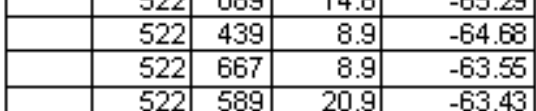

\begin{tabular}{|c|c|c|c|c|}
\hline & 522 & 589 & 20.9 & -63.43 \\
\hline & 522 & 493 & 6.0 & -61.53 \\
\hline
\end{tabular}

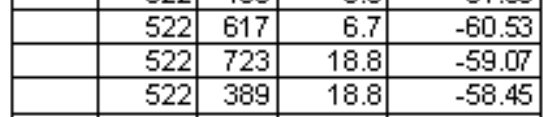

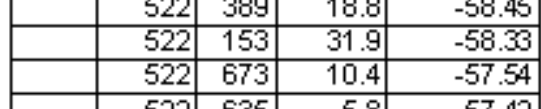

\begin{tabular}{|l|r|r|r|r|}
\hline & 522 & 635 & 5.8 & -57.42 \\
\hline $\mathrm{P}$ & 522 & 041 & 13.1 & -56.49 \\
\hline
\end{tabular}

\begin{tabular}{|l|r|r|r|r|}
\hline$P$ & 522 & 561 & 18.8 & -55.73 \\
\hline & 522 & 203 & 15.8 & -55.68 \\
\hline
\end{tabular}

\begin{tabular}{|r|r|r|r|r|}
\hline & 522 & 329 & 12.1 & -55.06 \\
\hline $\mathrm{P}$ & 522 & 711 & 9.6 & -52.88 \\
\hline
\end{tabular}

\begin{tabular}{|l|r|r|r|r|}
\hline$P$ & 522 & 101 & 9.9 & -52.63 \\
\hline$P$ & 522 & 461 & 9.0 & -52.33 \\
\hline
\end{tabular}


12,08 \% Change HLM Rank

Pilot NACS CompAge yrs. 1,05-109 Groupings HLM Count Mean Std.Dev Max Min

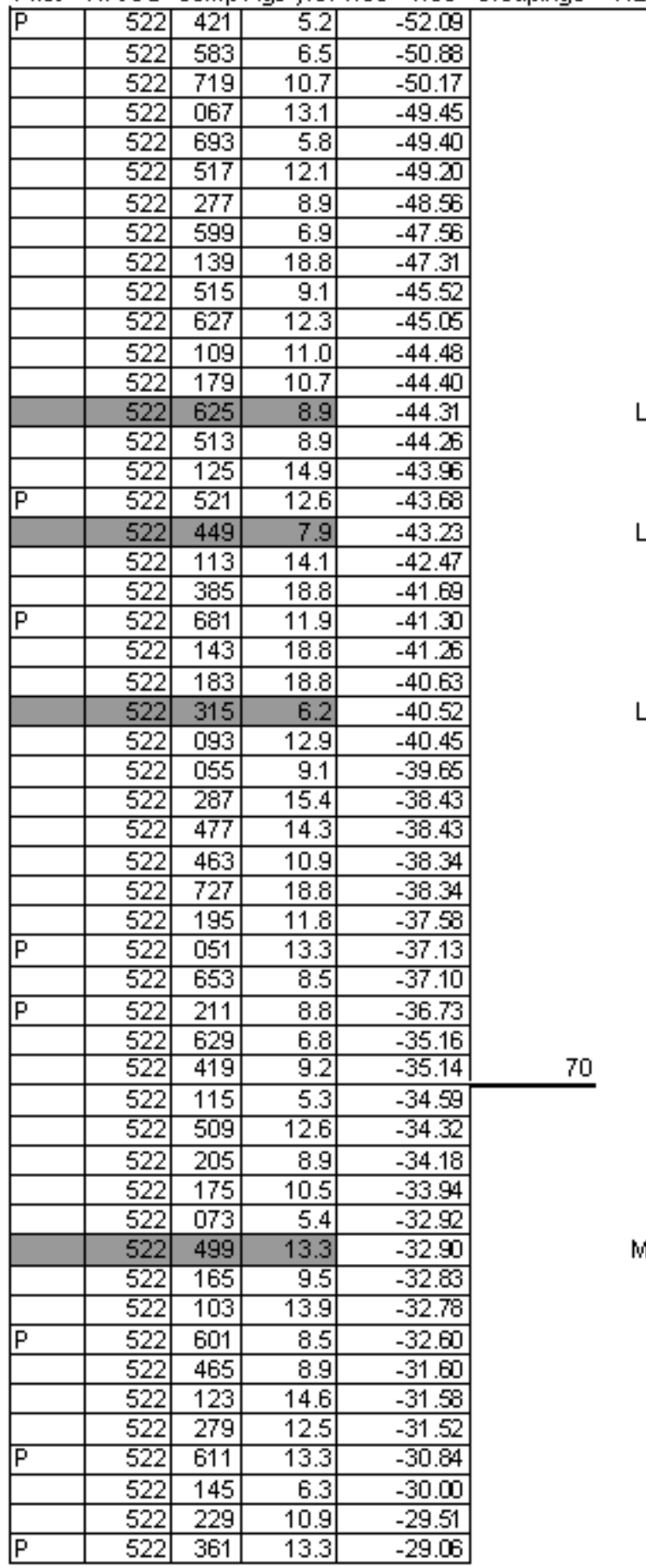


12,08 \% Change HLM Rank

Pilot NACS CompAge yrs. 1,05-109 Groupings HLM Count Mean Std.Dev Max Min

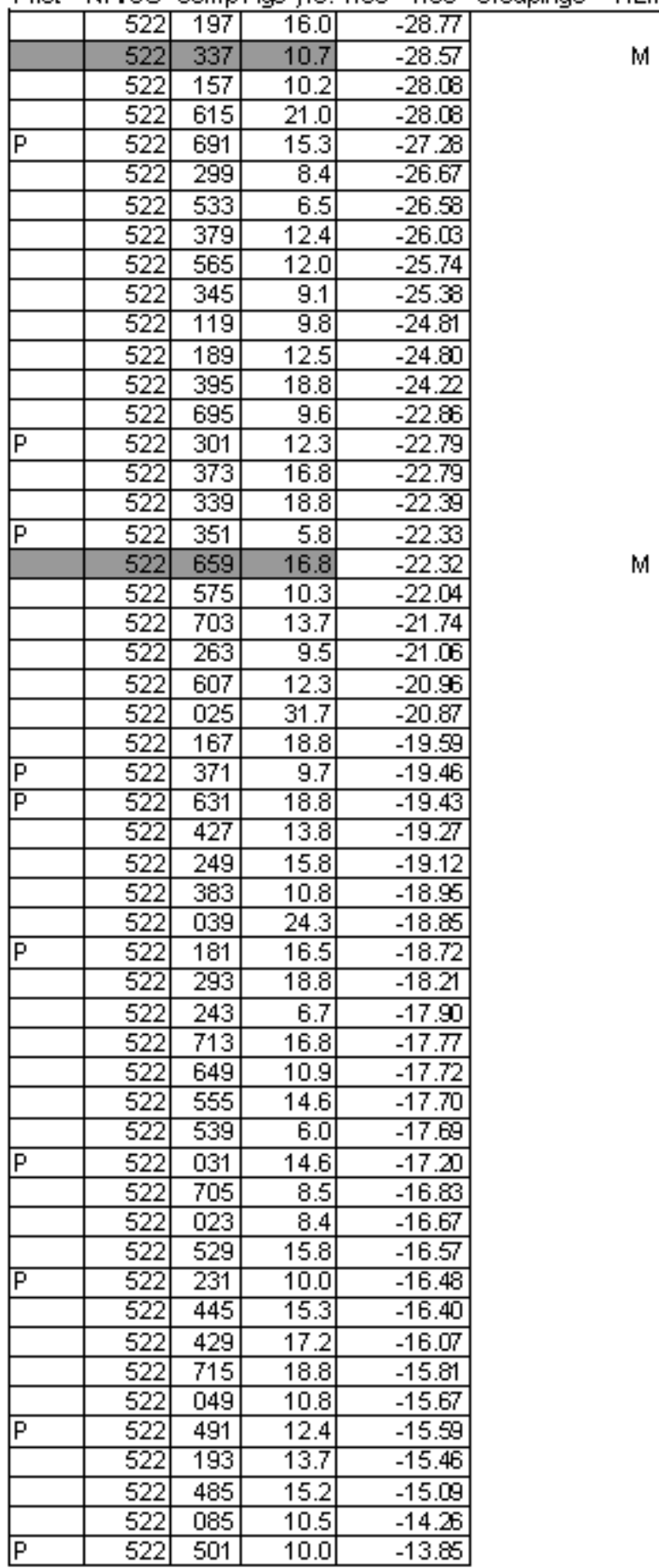


12008 \% Change HLM Rank

Pilot NAICS CompAge yrs. 1,05-1,09 Groupings HLM Count Mean Std.Dev Max Min

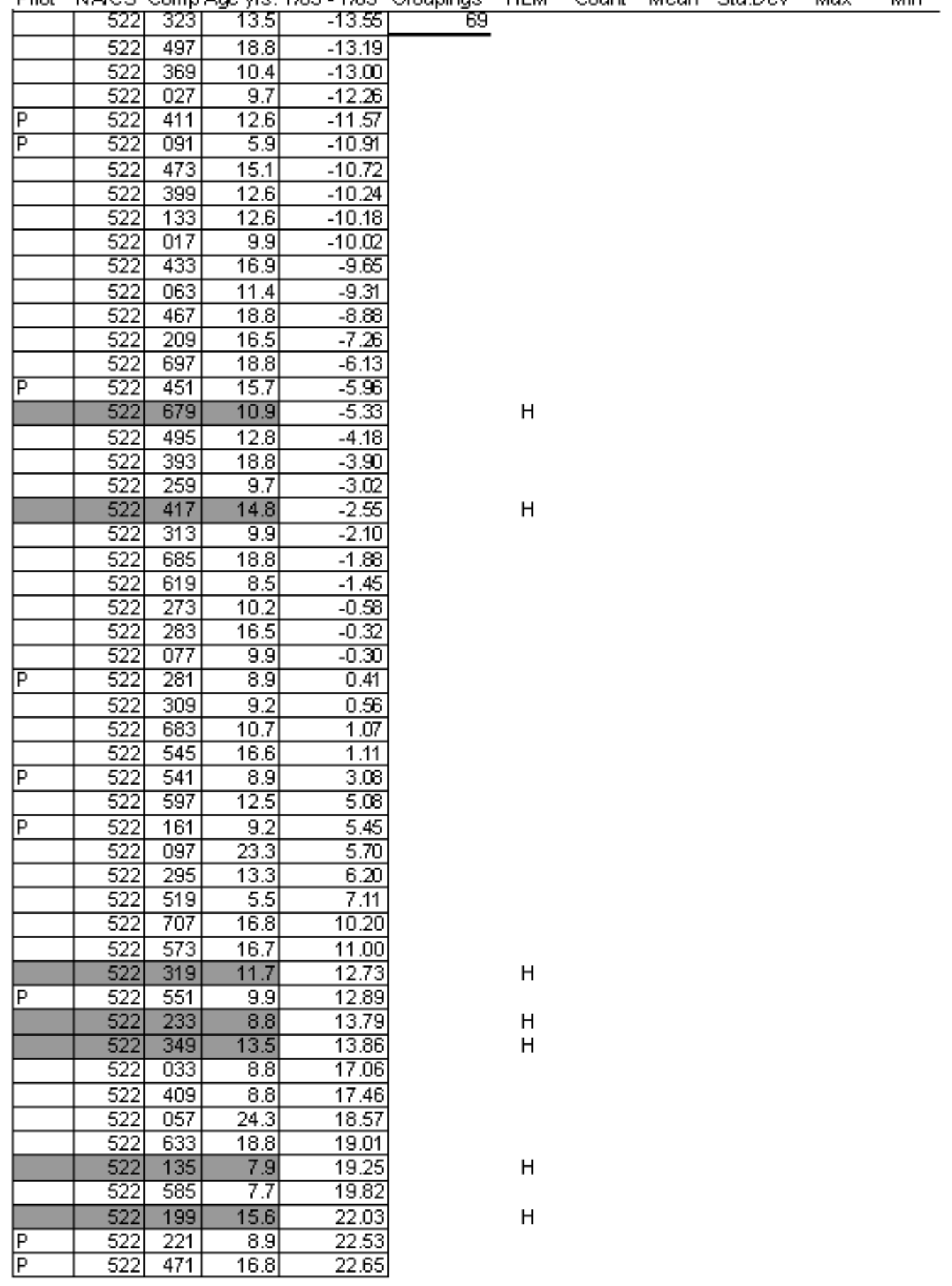


$12,08 \%$ Change HLM

Rank

Pilot NACS CompAge yrs. 1,05-1,09 Groupings HLM Count Mean Std.Dev Max Min

\begin{tabular}{|c|c|c|c|c|c|c|c|c|c|c|}
\hline $\mathrm{P}$ & 522 & 661 & 21.1 & 23.01 & & & & & & \\
\hline $\mathrm{P}$ & 522 & 311 & 10.7 & 23.22 & & & & & & \\
\hline & 522 & 523 & 9.7 & 23.62 & & & & & & \\
\hline & 522 & 185 & 24.3 & 24.30 & & & & & & \\
\hline & 522 & 547 & 10.1 & 25.02 & & & & & & \\
\hline & 522 & 579 & 11.9 & 25.76 & & & & & & \\
\hline & 522 & 677 & 9.8 & 28.94 & & & & & & \\
\hline & 522 & 563 & 10.4 & 33.91 & & & & & & \\
\hline & 522 & 447 & 11.0 & 34.92 & & & & & & \\
\hline \multirow[t]{4}{*}{$P$} & 522 & 721 & 17.0 & 36.89 & & & & & & \\
\hline & 522 & 605 & 10.6 & 39.54 & & & & & & \\
\hline & 522 & 047 & 8.4 & 47.57 & & & & & & \\
\hline & 522 & 359 & 17.5 & 50.11 & & $\mathrm{H}$ & & & & \\
\hline $\mathrm{P}$ & 522 & 061 & 22.2 & 59.34 & & & & & & \\
\hline \multirow[t]{3}{*}{$\bar{P}$} & 522 & 291 & 15.1 & 71.51 & & & & & & \\
\hline & 522 & 527 & 18.8 & 82.08 & & & & & & \\
\hline & 522 & 663 & 18.8 & 96.73 & & & & & & \\
\hline \multirow[t]{3}{*}{$\bar{P}$} & 522 & 651 & 18.8 & 99.58 & & & & & & \\
\hline & 522 & 403 & 7.6 & 193.00 & 70 & & 209 & -20.57 & 35.98 & $193.00-89.00$ \\
\hline & 524 & 126 & 15.2 & -96.61 & & & & & & \\
\hline
\end{tabular}

\begin{tabular}{|l|l|l|l|l|}
\hline & 524 & 008 & 17.4 & -94.09 \\
\hline & 524 & 102 & 13.7 & -91.01 \\
\hline
\end{tabular}

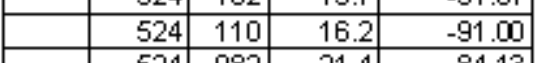

\begin{tabular}{|c|c|c|c|c|}
\hline & 524 & 082 & 21.4 & -84.13 \\
\hline & 524 & 012 & 16.8 & -61.49 \\
\hline
\end{tabular}

\begin{tabular}{|l|l|l|l|l|}
\hline & 524 & 052 & 23.8 & -59.16 \\
\hline & 524 & 070 & 18.8 & -48.36 \\
\hline
\end{tabular}

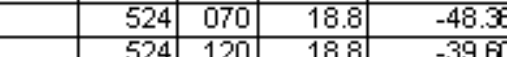

\begin{tabular}{|l|r|r|r|r|}
\hline$P$ & 524 & 056 & 16.8 & -32.25 \\
\hline
\end{tabular}

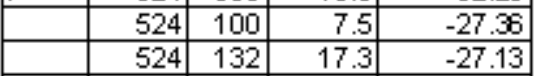

\begin{tabular}{|c|c|c|c|}
\hline & 52 & 00 & \\
\hline & $\frac{124}{524}$ & 09 & \\
\hline
\end{tabular}

\begin{tabular}{r|r|r|r|r|r|}
\cline { 1 - 3 } & 524 & 010 & 5.0 & -21.14 & 15 \\
\hline & 524 & 050 & 12.4 & -16.90 &
\end{tabular}

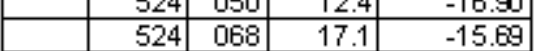

\begin{tabular}{|l|r|r|r|r|}
\hline & 524 & 034 & 18.8 & -14.61 \\
\hline$P$ & 524 & 106 & 22.4 & -11.61 \\
\hline
\end{tabular}

\begin{tabular}{|l|l|l|l|l|}
\hline $\mathrm{P}$ & 524 & 096 & 18.8 & -11.41 \\
\hline
\end{tabular}

\begin{tabular}{|l|r|r|r|r|} 
& 524 & 124 & 18.5 & -11.24 \\
\hline $\mathrm{P}$ & 524 & 066 & 17.1 & -8.48 \\
\hline
\end{tabular}

\begin{tabular}{|r|r|r|r|r|}
\hline & 524 & 066 & 17.1 & -8.40 \\
\hline & 524 & 040 & 17.7 & -3.54 \\
\hline & 524 & 122 & 21.0 & 2.3 \\
\hline
\end{tabular}

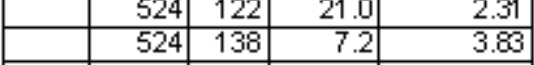

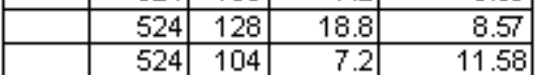

\begin{tabular}{|l|r|r|r|r|}
\hline$P$ & 524 & 116 & 18.8 & 22.46 \\
\hline
\end{tabular}

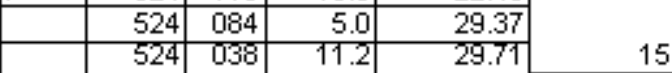

\begin{tabular}{|l|r|r|r|r|}
\hline & 524 & 118 & 9.7 & 31.35 \\
\hline $\mathrm{P}$ & 524 & 026 & 12.6 & 34.74 \\
\hline $\mathrm{P}$ & 524 & 086 & 8.5 & 35.64 \\
\hline
\end{tabular} 
12,08 \% Change HLM Rank

Pilot NAlCS CompAge yrs. 1,05-1,09 Groupings HLM Count Mean Std.Dev Max Min

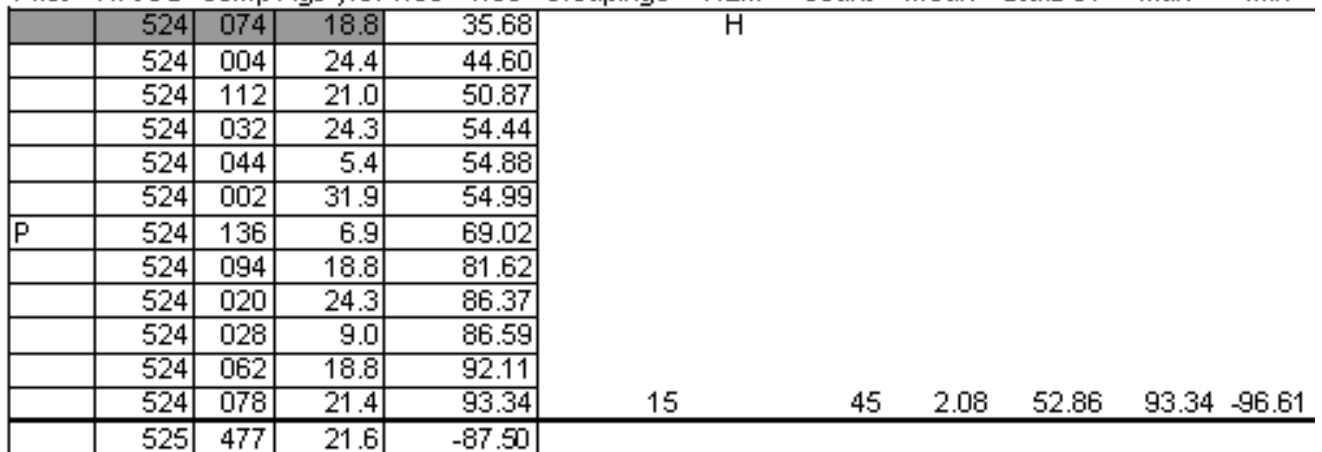

\begin{tabular}{l|r|r|r|r|}
\hline 525 & 333 & 10.7 & -83.78 \\
\hline
\end{tabular}

\begin{tabular}{|l|r|r|r|r|}
\hline$P$ & 525 & 815 & 10.9 & -79.40 \\
\hline
\end{tabular}

\begin{tabular}{|r|r|r|r|}
\hline 525 & 703 & 10.9 & -66.17 \\
\hline
\end{tabular}

\begin{tabular}{|l|r|r|r|r|}
\hline $\mathrm{P}$ & 525 & 445 & 5.5 & -52.78 \\
\hline
\end{tabular}

\begin{tabular}{|l|l|l|l|l|}
\hline & 525 & 043 & 5.3 & -43.65 \\
\hline & 525 & 777 & 5.1 & -40.40 \\
\hline
\end{tabular}

\begin{tabular}{|l|r|r|r|r|}
\hline$P$ & 525 & 145 & 22.4 & -37.95 \\
\hline
\end{tabular}

\begin{tabular}{|l|l|l|l|l|}
\hline & 525 & 709 & 13.3 & -37.24 \\
\hline
\end{tabular}

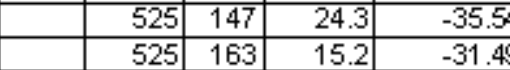

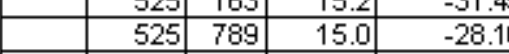

\begin{tabular}{|l|r|r|r|r|}
\hline & 525 & 039 & 10.8 & -27.69 \\
\hline $\mathrm{P}$ & 525 & 385 & 20.9 & -27.30 \\
\hline
\end{tabular}

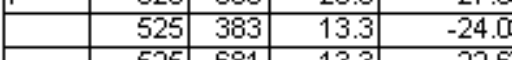

\begin{tabular}{|l|r|r|r|r|} 
& 525 & 681 & 13.3 & -22.67 \\
\hline $\mathrm{P}$ & 525 & 315 & 5.2 & -15.40 \\
\hline
\end{tabular}

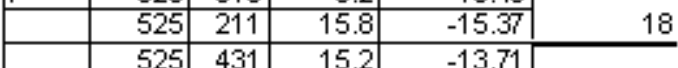

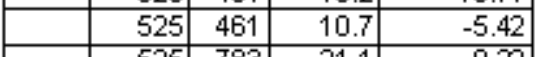

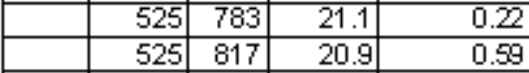

\begin{tabular}{|l|r|r|r|r|}
\hline$P$ & 525 & 165 & 15.8 & 3.74 \\
\hline & 525 & 011 & 14.7 & 8.00 \\
\hline
\end{tabular}

\begin{tabular}{l|l|l|l|}
525 & 479 & 10.0 & 8.00 \\
\hline
\end{tabular}

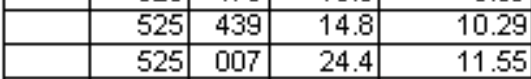

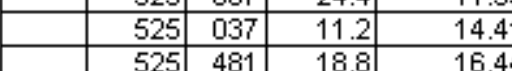

\begin{tabular}{|c|c|c|c|}
\hline & 525 & 481 & 18.8 \\
\hline & 525 & 711 & 13.3 \\
\hline
\end{tabular}

\begin{tabular}{rr|r|r|}
\hline 525 & 387 & 16.8 & 18.29 \\
\hline
\end{tabular}

\begin{tabular}{|l|r|r|r|r|}
\hline $\mathrm{P}$ & 525 & 035 & 5.2 & 18.82 \\
\hline
\end{tabular}

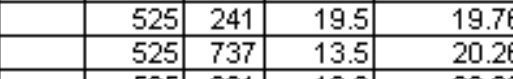

\begin{tabular}{|l|r|r|r|r|r}
\hline & 525 & 291 & 16.3 & 26.23 & \multirow{1}{*}{18} \\
\hline & 525 & 401 & 11.2 & 29.72 & \\
\hline & 525 & 023 & 11.1 & 30.68 & \\
\hline
\end{tabular}

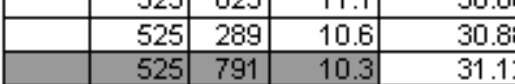

\begin{tabular}{|l|r|r|r|r|}
\hline $\mathrm{P}$ & 525 & 455 & 12.6 & 31.16 \\
\hline
\end{tabular} 
$12,08 \%$ Rank

Pilot NAJCS CompAge yrs. 1,05-1,09 Groupings HLM Count Mean Std.Dev Max Min

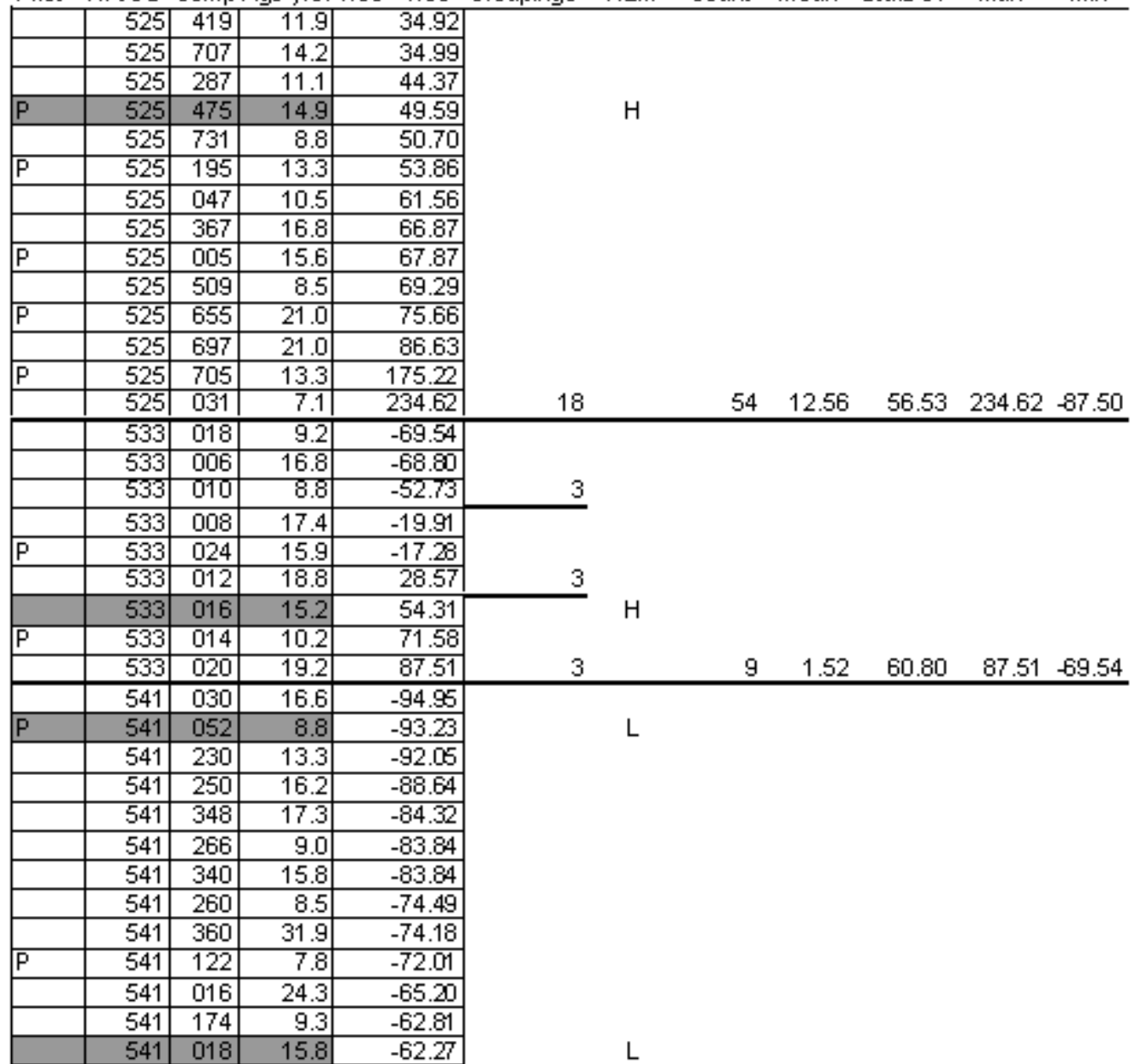

\begin{tabular}{|r|r|r|r|r|}
\hline$P$ & 541 & 268 & 7.3 & -62.14 \\
\hline$P$ & 541 & 022 & 11.9 & -61.8 \\
\hline
\end{tabular}

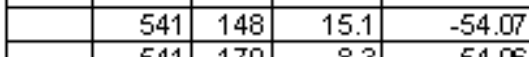

\begin{tabular}{l|r|r|r}
541 & 170 & 8.3 & -54.06 \\
\hline
\end{tabular}

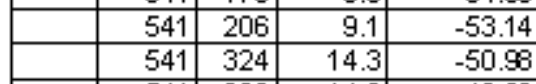

\begin{tabular}{|r|r|r|r|}
\hline 541 & 226 & 14.9 & -48.03 \\
\hline
\end{tabular}

\begin{tabular}{|l|r|r|r|r|}
\hline$P$ & 541 & 212 & 9.0 & -44.61 \\
\hline
\end{tabular}

\begin{tabular}{r|r|r|r|r|}
\hline & 541 & 358 & 18.8 & -43.57 \\
\hline & 541 & 086 & 8.8 & -42.44 \\
\hline
\end{tabular}

\begin{tabular}{|l|r|r|r|r|}
\hline $\mathrm{P}$ & 541 & 192 & 16.7 & -41.95 \\
\hline
\end{tabular}

\begin{tabular}{l|r|r|r|r|}
\hline & 541 & 100 & 11.9 & -41.37 \\
\hline & 541 & 128 & 16.8 & -38.17 \\
\hline
\end{tabular}

\begin{tabular}{|l|r|r|r|r|}
\hline $\mathrm{P}$ & 541 & 082 & 9.8 & -37.05 \\
\hline
\end{tabular}

\begin{tabular}{r|r|r|r|}
541 & 068 & 14.8 & -33.30 \\
\hline
\end{tabular}

\begin{tabular}{l|l|l|l|}
541 & 056 & 5.1 & -32.28 \\
\hline
\end{tabular} 
12,08 \% Change HLM Rank

Pilot NASCS CompAgeyrs. 1,05-1,09 Groupings HLM Count Mean Std.Dev Max Min

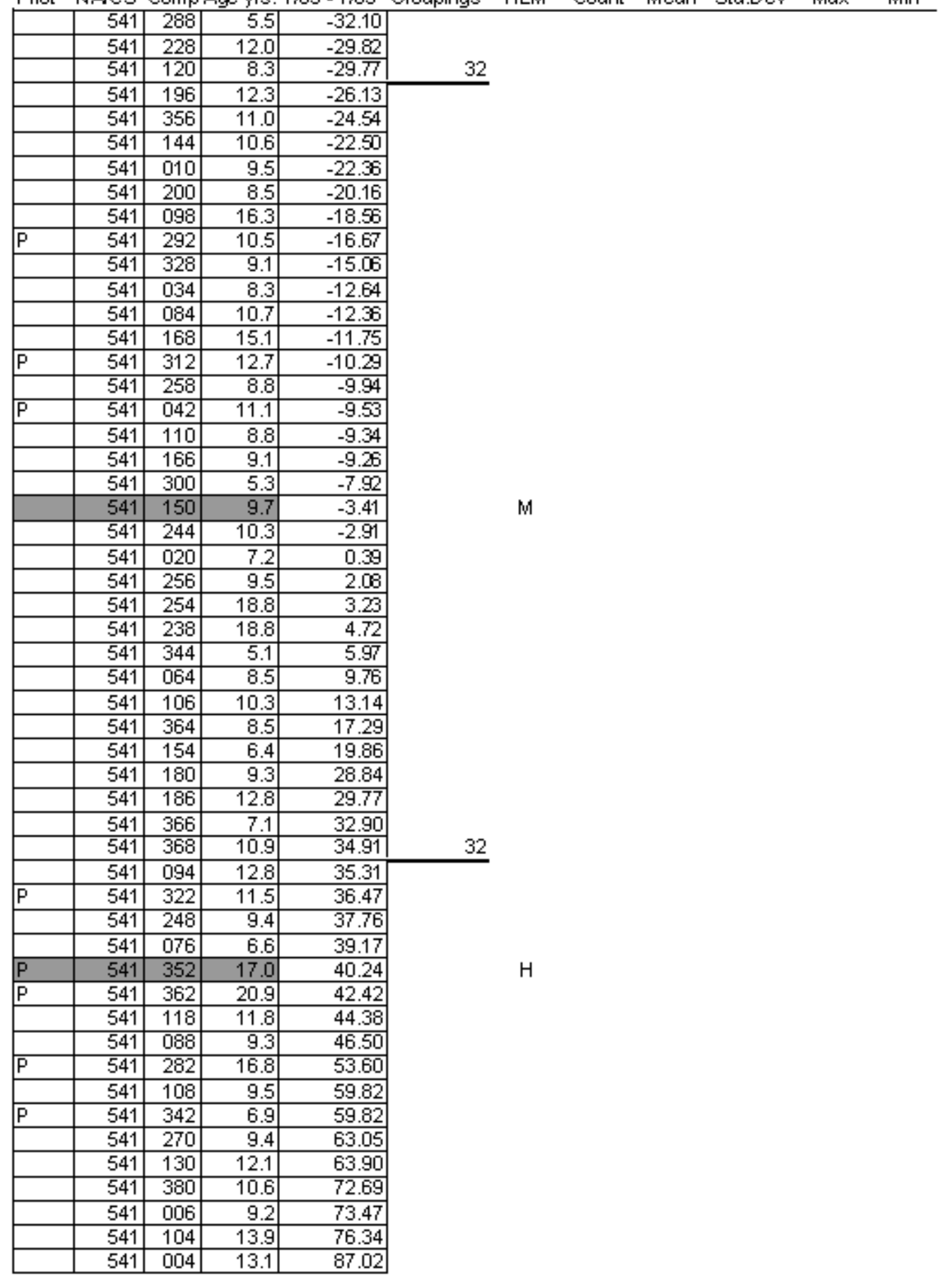


12008 \% Change HLM Rank

Pilot NAICS CompAge yrs. 1,05-1,09 Groupings HLM Count Mean Std.Dev Max Min

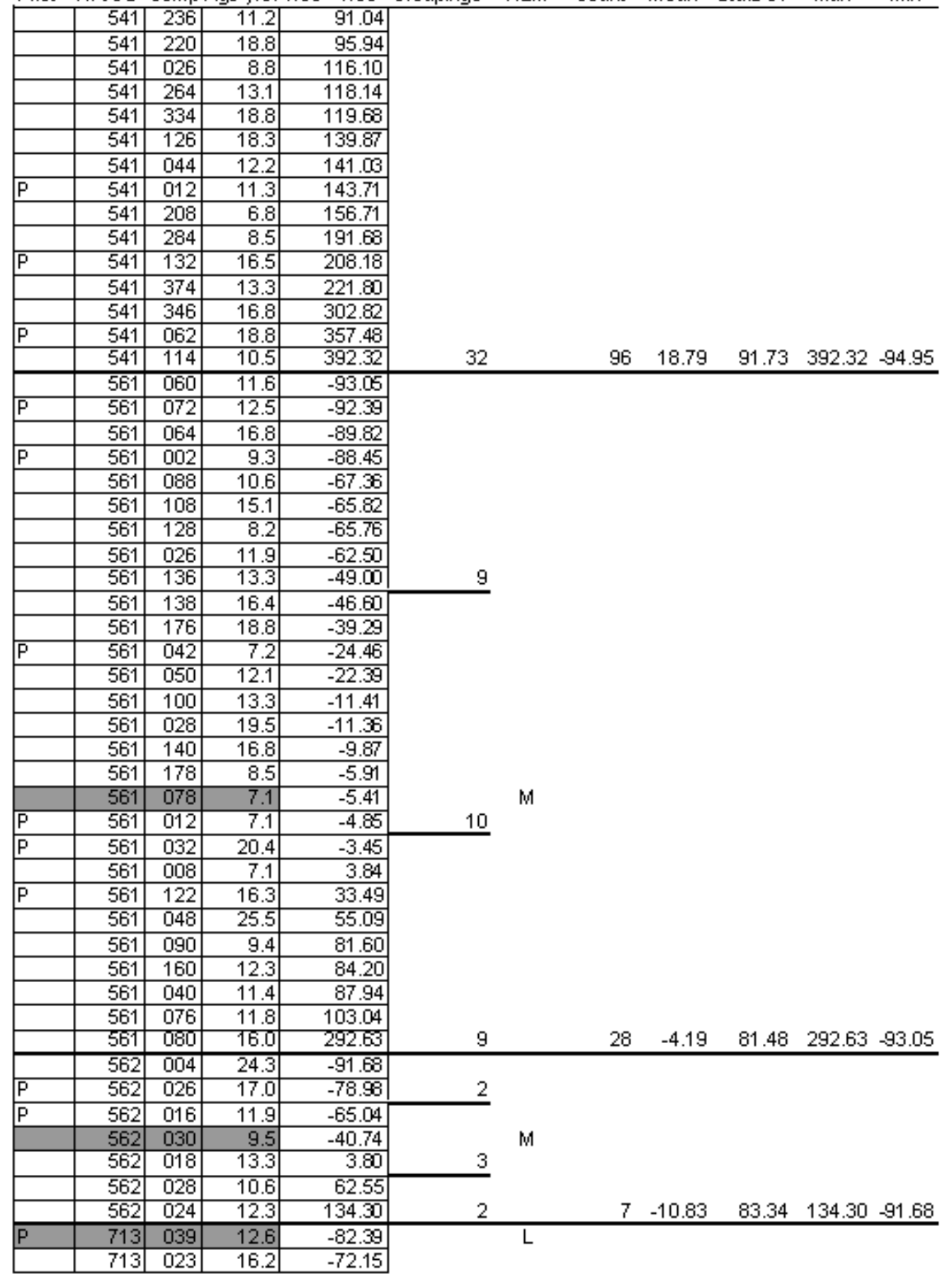


$12,08 \%$ Rank Pilot NalCS CompAge yrs. 1,05-1,09 Groupings HLM Count Mean Std.Dev Max Min

\begin{tabular}{|c|c|c|c|c|c|c|c|c|c|c|}
\hline & 713 & 051 & 9.8 & -69.67 & & & & & & \\
\hline \multirow[t]{2}{*}{$\mathrm{P}$} & 713 & 029 & 16.0 & -59.29 & 4 & & & & & \\
\hline & 713 & 007 & 15.2 & -57.74 & & & & & & \\
\hline \multirow[t]{12}{*}{$\bar{P}$} & 713 & 009 & 15.1 & -55.13 & & & & & & \\
\hline & 713 & 005 & 8.8 & -37.74 & & & & & & \\
\hline & 713 & 003 & 15.1 & -13.25 & & & & & & \\
\hline & 713 & 015 & 12.2 & -1.63 & 5 & & & & & \\
\hline & 713 & 013 & 46.9 & 20.00 & & & & & & \\
\hline & 713 & 035 & 14.6 & 57.33 & & & & & & \\
\hline & 713 & 047 & 11.8 & 101.99 & & & & & & \\
\hline & 713 & 017 & 15.3 & 123.86 & 4 & & 13 & -11.22 & 68.39 & $123.86-82.39$ \\
\hline & 722 & 028 & 15.1 & -78.74 & & & & & & \\
\hline & 722 & 020 & 6.1 & -65.99 & & & & & & \\
\hline & 722 & 066 & 16.8 & -62.81 & & & & & & \\
\hline & 722 & 052 & 17.9 & -52.36 & & & & & & \\
\hline \multirow[t]{3}{*}{$\bar{P}$} & 722 & 054 & 10.0 & -50.63 & & $\mathrm{~L}$ & & & & \\
\hline & 722 & 016 & 16.3 & -49.05 & & & & & & \\
\hline & 722 & 060 & 9.5 & -44.70 & 7 & & & & & \\
\hline \multirow[t]{4}{*}{$\mathrm{P}$} & 722 & 004 & 24.3 & -38.13 & & & & & & \\
\hline & 722 & 058 & 6.4 & -31.45 & & & & & & \\
\hline & 722 & 008 & 19.4 & -28.49 & & & & & & \\
\hline & 722 & 030 & 12.1 & -19.85 & & & & & & \\
\hline \multirow[t]{2}{*}{$\mathrm{P}$} & 722 & 014 & 18.8 & -18.65 & & & & & & \\
\hline & 722 & 018 & 18.8 & -8.66 & 6 & & & & & \\
\hline \multirow[t]{3}{*}{$\mathrm{P}$} & 722 & 034 & 13.3 & -8.05 & & & & & & \\
\hline & 722 & 006 & 24.3 & -1.05 & & & & & & \\
\hline & 722 & 032 & 24.3 & 0.69 & & & & & & \\
\hline \multirow[t]{4}{*}{$\mathrm{P}$} & 722 & 044 & 23.4 & 3.05 & & & & & & \\
\hline & 722 & 022 & 13.6 & 27.33 & & & & & & \\
\hline & 722 & 050 & 15.8 & 126.54 & & & & & & \\
\hline & 722 & 046 & 38.9 & 127.07 & 7 & & 20 & -13.70 & 55.02 & $127.07 \quad-78.74$ \\
\hline \multirow[t]{9}{*}{$\mathrm{P}$} & 812 & 026 & 17.0 & -98.72 & & & & & & \\
\hline & 812 & 022 & 12.4 & -49.44 & & & & & & \\
\hline & 812 & 018 & 17.5 & -46.53 & & & & & & \\
\hline & 812 & 024 & 17.2 & -7.81 & 4 & & & & & \\
\hline & 812 & 008 & 19.5 & -6.29 & & & & & & \\
\hline & 812 & 030 & 7.1 & -3.28 & & & & & & \\
\hline & 812 & 004 & 12.1 & 3.60 & 3 & & & & & \\
\hline & 812 & 012 & 8.5 & 13.22 & & & & & & \\
\hline & 812 & 002 & 24.3 & 18.40 & & & & & & \\
\hline \multirow[t]{2}{*}{$\bar{P}$} & 812 & 006 & 11.4 & 33.68 & & $\mathrm{H}$ & & & & \\
\hline & 812 & 028 & 20.9 & 34.99 & 4 & $\mathrm{H}$ & 11 & -9.83 & 40.36 & $34.99 \quad-98.72$ \\
\hline \multicolumn{2}{|c|}{ Count } & & 1290 & 1288 & & & 1288 & & & \\
\hline \multicolumn{2}{|c|}{ Mean } & & 14.66 & 9.88 & & & & & & \\
\hline \multicolumn{2}{|c|}{ Std. Dev } & & 6.17 & 105.54 & & & & & & \\
\hline \multicolumn{2}{|c|}{ Median } & & 13.67 & -13.22 & & & & & & \\
\hline \multicolumn{2}{|c|}{$\operatorname{Max}$} & & 46.92 & 1007.75 & & & & & & \\
\hline \multicolumn{2}{|c|}{ Min } & & 5.00 & -99.62 & & & & & & \\
\hline
\end{tabular}




\section{Appendix N: Dependent Variable by Responding Companies Only}

\begin{tabular}{|c|c|c|c|c|c|}
\hline Pilot & W. & Co. No. & Age yts. & $1,05-1,09$ & Performance \\
\hline$\overline{\mathrm{P}}$ & 541 & 052 & 8.6 & -9323 & \multirow{24}{*}{ L } \\
\hline $\mathrm{P}$ & 713 & 0139 & 12.6 & -62.39 & \\
\hline & 221 & 001 & 8.3 & -72.93 & \\
\hline $\mathrm{P}$ & 325 & 153 & 8.5 & 69.66 & \\
\hline & 211 & DEG & 6.6 & -66.05 & \\
\hline & 541 & 018 & 158 & -62.27 & \\
\hline $\mathrm{P}$ & 311 & 0.58 & 18.6 & -61.97 & \\
\hline & 441 & 025 & 210 & -61.46 & \\
\hline & 325 & 035 & 20.5 & 56.57 & \\
\hline & 334 & Ei]1 & 21.8 & -51.82 & \\
\hline & 515 & 009 & 8.5 & -51.56 & \\
\hline $\mathrm{P}$ & 722 & 0.54 & 10.0 & 50.63 & \\
\hline \multirow[t]{15}{*}{$\mathrm{P}$} & 325 & 453 & 5.2 & 46.45 & \\
\hline & 312 & 033 & 8.8 & 45.67 & \\
\hline & 522 & 625 & 8.9 & 44.31 & \\
\hline & 522 & 449 & 79 & 4323 & \\
\hline & 562 & 030 & 9.5 & 40.74 & \\
\hline & 522 & 315 & 6.2 & 40.52 & \\
\hline & 237 & 027 & 11.9 & 40.21 & \\
\hline & 334 & 499 & 24.3 & -34.99 & \\
\hline & 522 & 499 & 133 & 32.90 & \\
\hline & 334 & 439 & 14.4 & 30.14 & \\
\hline & 333 & 130 & 138 & -29.69 & \\
\hline & 522 & 337 & 10.7 & -28.57 & \\
\hline & 511 & 159 & 8.2 & -23.97 & \multirow{24}{*}{ 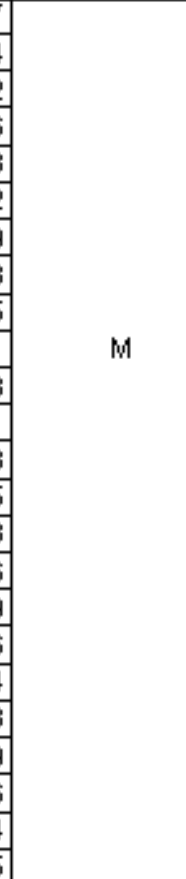 } \\
\hline & 311 & 084 & 133 & -22.94 & \\
\hline & 522 & 659 & 16.8 & -22.32 & \\
\hline \multirow[t]{9}{*}{$\mathrm{P}$} & 336 & 048 & 14.4 & -18.66 & \\
\hline & 332 & 0107 & 9.3 & -15.19 & \\
\hline & 511 & 019 & 9.5 & -14.22 & \\
\hline & 333 & 104 & 23.4 & -12.99 & \\
\hline & 511 & 0105 & 138 & -9.13 & \\
\hline & 443 & D106: & 11.4 & -6.95 & \\
\hline & 561 & 078 & 7.1 & -5.41 & \\
\hline & 522 & 679 & 10.9 & -5.33 & \\
\hline & 541 & 150 & 9.7 & -3.41 & \\
\hline \multirow[t]{5}{*}{$\mathrm{P}$} & 423 & 135 & 13.4 & -3.13 & \\
\hline & 522 & 417 & 14.8 & -2.55 & \\
\hline & 339 & 0187 & 16.8 & -0.23 & \\
\hline & 325 & 277 & 8.5 & 3.56 & \\
\hline & 334 & 131 & 18.8 & 4.49 & \\
\hline \multirow[t]{6}{*}{$\mathrm{P}$} & 237 & 민ㅁ & 10.2 & 5.56 & \\
\hline & 334 & 477 & 11.0 & 12.64 & \\
\hline & 522 & 319 & 11.7 & 12.73 & \\
\hline & 522 & 233 & 8.8 & 13.79 & \\
\hline & 522 & 349 & 13.5 & 13.86 & \\
\hline & 221 & 049 & 21.1 & 15.34 & \\
\hline $\bar{P}$ & 325 & 303 & 9.8 & 15.55 & \\
\hline
\end{tabular}




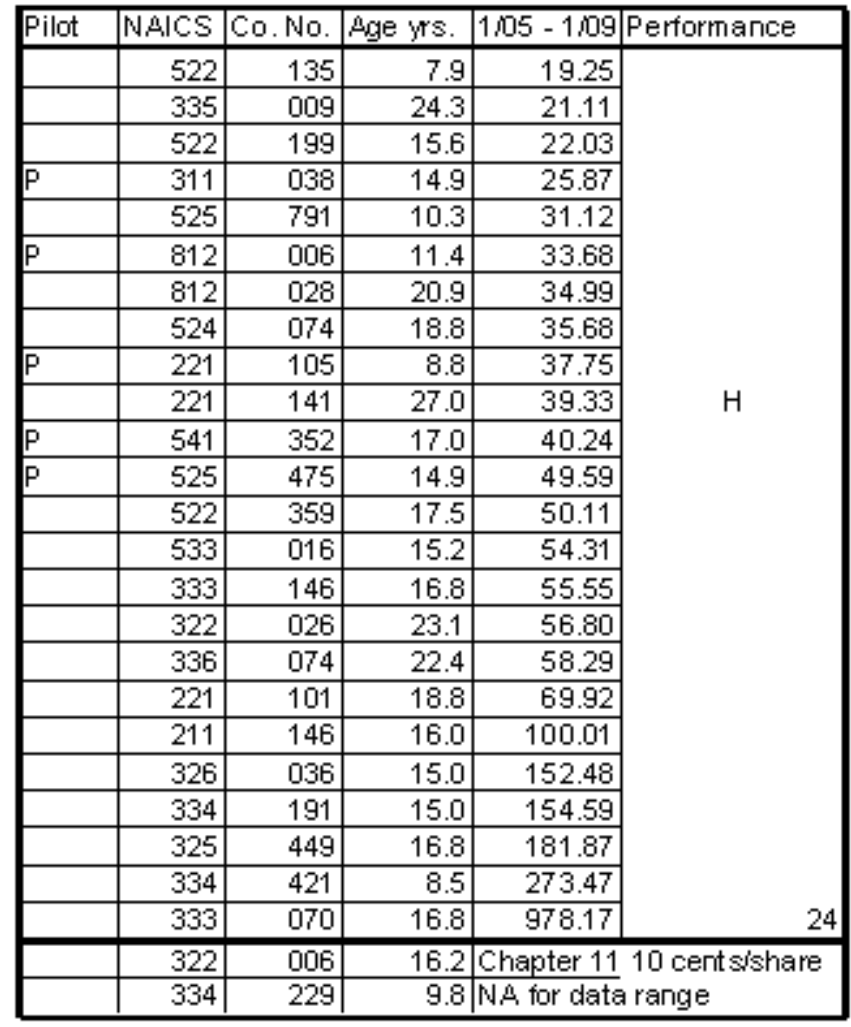

\begin{tabular}{lrr|}
\hline Count & 74 & 72 \\
Mean & 13.81 & 17.63 \\
Std. Dev. & 5.09 & 130.42 \\
Wedian & 13.46 & -3.27 \\
Max & 27.00 & 978.17 \\
Win & 5.17 & -93.23 \\
\hline
\end{tabular}




\section{Appendix O: Process Ranking Conversion $\mathrm{H}_{1}$}

\begin{tabular}{|c|c|c|c|c|c|c|c|c|c|}
\hline $\begin{array}{ll} & 1 \mathrm{H}, 2 \mathrm{M} \\
\mathrm{R} \in \mathrm{P}, 3 \mathrm{~L} \\
\mathrm{ID} & \mathrm{R} \text { ank } \\
\end{array}$ & Company & $\begin{array}{l}\text { Process } \\
\text { Rark } 1 \\
\text { (abreviated) }\end{array}$ & $\begin{array}{l}\text { Process } \\
\text { Rark } 2 \\
\text { (abreviated) }\end{array}$ & $\begin{array}{l}\text { Process } \\
\text { Rank } 3 \\
\text { (abreviated) }\end{array}$ & $\begin{array}{l}\text { Process } \\
\text { Rank } 4 \\
\text { (abreviated) }\end{array}$ & $\begin{array}{l}\text { Identity } \\
\text { Process } \\
\text { Rank }\end{array}$ & $\begin{array}{l}\text { Priority } \\
\text { Process } \\
\text { Rank }\end{array}$ & $\begin{array}{l}\text { Back ground } \\
\text { Process } \\
\text { Rark }\end{array}$ & $\begin{array}{l}\text { Mandated } \\
\text { Process } \\
\text { Rank }\end{array}$ \\
\hline $22 \mathrm{M}$ & 3360.048 & 1 & $B$ & $M$ & $\mathrm{P}$ & 1 & 4 & 2 & 3 \\
\hline $32 \mathrm{M}$ & 4230.135 & P & $B$ & 1 & $M$ & 3 & 1 & 2 & 4 \\
\hline $43 \mathrm{~L}$ & 7220.054 & I & $\mathrm{P}$ & B & $M$ & 1 & 2 & 3 & 4 \\
\hline $52 \mathrm{M}$ & 4230.135 & $M$ & B & 1 & $\mathrm{P}$ & 3 & 4 & 2 & 1 \\
\hline $61 \mathrm{H}$ & 5250.475 & $\mathrm{P}$ & B & 1 & $M$ & 3 & 1 & 2 & 4 \\
\hline $73 \mathrm{~L}$ & 7130.039 & I & B & $\mathrm{P}$ & $M$ & 1 & 3 & 2 & 4 \\
\hline $81 \mathrm{H}$ & 8120.006 & $\mathrm{P}$ & $M$ & B & 1 & 4 & 1 & 3 & 2 \\
\hline $93 \mathrm{~L}$ & 5410.052 & 1 & $\mathrm{P}$ & B & $M$ & 1 & 2 & 3 & 4 \\
\hline $101 \mathrm{H}$ & 5410.352 & $\mathrm{P}$ & $M$ & B & 1 & 4 & 1 & 3 & 2 \\
\hline $111 \mathrm{H}$ & 3250.303 & $B$ & $M$ & $\mathrm{P}$ & 1 & 4 & 3 & 1 & 2 \\
\hline $122 \mathrm{M}$ & 2210.105 & 1 & $\mathrm{P}$ & B & $M$ & 1 & 2 & 3 & 4 \\
\hline $132 \mathrm{M}$ & 2370.001 & $B$ & $\mathrm{P}$ & $M$ & 1 & 4 & 2 & 1 & 3 \\
\hline $141 \mathrm{H}$ & 3110.038 & B & $M$ & $\mathrm{P}$ & 1 & 4 & 3 & 1 & 2 \\
\hline $153 \mathrm{~L}$ & 3250.153 & $B$ & $\mathrm{P}$ & 1 & $M$ & 3 & 2 & 1 & 4 \\
\hline $163 \mathrm{~L}$ & 3110.058 & B & $M$ & 1 & P & 3 & 4 & 1 & 2 \\
\hline $173 \mathrm{~L}$ & 3330.104 & $M$ & B & 1 & $\mathrm{P}$ & 3 & 4 & 2 & 1 \\
\hline $183 \mathrm{~L}$ & 3110.084 & $\mathrm{~B}$ & $M$ & $\mathrm{P}$ & 1 & 4 & 3 & 1 & 2 \\
\hline $192 \mathrm{M}$ & 5110.159 & I & B & $\mathrm{P}$ & $M$ & 1 & 3 & 2 & 4 \\
\hline $203 \mathrm{~L}$ & 5220.625 & $M$ & B & $\mathrm{P}$ & 1 & 4 & 3 & 2 & 1 \\
\hline $213 L$ & 5220.315 & $M$ & 1 & $\mathrm{P}$ & B & 2 & 3 & 4 & 1 \\
\hline $222 \mathrm{M}$ & 5620.030 & $M$ & 1 & $\mathrm{P}$ & B & 2 & 3 & 4 & 1 \\
\hline $231 \mathrm{H}$ & 5220.349 & 1 & $\mathrm{P}$ & $M$ & B & 1 & 2 & 4 & 3 \\
\hline $241 \mathrm{H}$ & 2210.101 & $M$ & $\mathrm{P}$ & 1 & B & 3 & 2 & 4 & 1 \\
\hline $253 \mathrm{~L}$ & 3120.033 & $\mathrm{P}$ & B & $M$ & 1 & 4 & 1 & 2 & 3 \\
\hline $262 \mathrm{M}$ & 3340.439 & B & $\mathrm{P}$ & 1 & $M$ & 3 & 2 & 1 & 4 \\
\hline $291 \mathrm{H}$ & 5330.016 & B & 1 & P & $M$ & 2 & 3 & 1 & 4 \\
\hline $301 \mathrm{H}$ & 5220.319 & 1 & $M$ & $\mathrm{P}$ & B & 1 & 3 & 4 & 2 \\
\hline $31 \mathrm{HH}$ & 3330.070 & $\mathrm{P}$ & B & 1 & $M$ & 3 & 1 & 2 & 4 \\
\hline $32 \mathrm{2M}$ & 3340.499 & B & $M$ & 1 & $\mathrm{P}$ & 3 & 4 & 1 & 2 \\
\hline $332 \mathrm{M}$ & 3340.601 & 1 & B & $M$ & $\mathrm{P}$ & 1 & 4 & 2 & 3 \\
\hline $343 L$ & 2210.049 & $\mathrm{P}$ & $M$ & B & 1 & 4 & 1 & 3 & 2 \\
\hline $351 \mathrm{H}$ & 5220.359 & $\mathrm{P}$ & 1 & B & $M$ & 2 & 1 & 3 & 4 \\
\hline $361 \mathrm{H}$ & 5240.074 & $\mathrm{P}$ & 1 & $M$ & B & 2 & 1 & 4 & 3 \\
\hline $371 \mathrm{H}$ & 5250.791 & $B$ & $M$ & $\mathrm{P}$ & 1 & 4 & 3 & 1 & 2 \\
\hline $382 \mathrm{M}$ & 5220.337 & $M$ & $\mathrm{P}$ & 1 & B & 3 & 2 & 4 & 1 \\
\hline $391 \mathrm{H}$ & 5220.135 & 1 & $M$ & $\mathrm{P}$ & $B$ & 1 & 3 & 4 & 2 \\
\hline $401 \mathrm{H}$ & 3360.074 & $\mathrm{P}$ & $M$ & B & 1 & 4 & 1 & 3 & 2 \\
\hline $412 \mathrm{M}$ & 3390.087 & $\mathrm{P}$ & $M$ & $\mathrm{~B}$ & 1 & 4 & 1 & 3 & 2 \\
\hline $421 \mathrm{H}$ & 3340.421 & $\mathrm{P}$ & $M$ & B & 1 & 4 & 1 & 3 & 2 \\
\hline $432 \mathrm{M}$ & 5150.009 & $\mathrm{P}$ & 1 & B & $M$ & 2 & 1 & 3 & 4 \\
\hline $442 \mathrm{M}$ & 3250.277 & $M$ & $\mathrm{P}$ & 1 & B & 3 & 2 & 4 & 1 \\
\hline $451 \mathrm{H}$ & 3220.026 & B & 1 & $M$ & $\mathrm{P}$ & 2 & 4 & 1 & 3 \\
\hline $463 \mathrm{~L}$ & 2370.027 & B & $M$ & $\mathrm{P}$ & 1 & 4 & 3 & 1 & 2 \\
\hline $473 \mathrm{~L}$ & 5410.018 & $\mathrm{P}$ & $M$ & B & 1 & 4 & 1 & 3 & 2 \\
\hline $481 \mathrm{H}$ & 5220.199 & $B$ & $M$ & P & 1 & 4 & 3 & 1 & 2 \\
\hline $491 \mathrm{H}$ & 5220.679 & $M$ & B & $\mathrm{P}$ & 1 & 4 & 3 & 2 & 1 \\
\hline $503 \mathrm{~L}$ & 3320.007 & $\mathrm{~B}$ & $M$ & $\mathrm{P}$ & 1 & 4 & 3 & 1 & 2 \\
\hline $513 \mathrm{~L}$ & 2110.060 & B & 1 & $\mathrm{P}$ & $M$ & 2 & 3 & 1 & 4 \\
\hline $521 \mathrm{H}$ & 3340.191 & B & 1 & $P$ & $M$ & 2 & 3 & 1 & 4 \\
\hline $531 \mathrm{H}$ & 5220.233 & $M$ & B & $\mathrm{P}$ & 1 & 4 & 3 & 2 & 1 \\
\hline $543 \mathrm{~L}$ & 5220.449 & $M$ & 1 & $\mathrm{P}$ & B & 2 & 3 & 4 & 1 \\
\hline $552 \mathrm{M}$ & 5110.019 & 1 & $\mathrm{P}$ & B & $M$ & 1 & 2 & 3 & 4 \\
\hline $562 \mathrm{M}$ & 3330.145 & $\mathrm{P}$ & 1 & $M$ & B & 2 & 1 & 4 & 3 \\
\hline $572 \mathrm{M}$ & 5220.659 & $M$ & $\mathrm{P}$ & 1 & B & 3 & 2 & 4 & 1 \\
\hline $583 \mathrm{~L}$ & 2210.001 & $\mathrm{P}$ & B & 1 & $M$ & 3 & 1 & 2 & 4 \\
\hline
\end{tabular}




\begin{tabular}{|c|c|c|c|c|c|c|c|c|c|c|}
\hline $\begin{array}{l}R \in P \\
\text { ID }\end{array}$ & $\begin{array}{l}1 \mathrm{H}, 2 \mathrm{M} \\
3 \mathrm{~L} \\
\mathrm{R} \text { ank }\end{array}$ & Company & $\begin{array}{l}\text { Process } \\
\text { Rark } 1 \\
\text { (abreviated) }\end{array}$ & $\begin{array}{l}\text { Process } \\
\text { Rark } 2 \\
\text { (abreviated) }\end{array}$ & $\begin{array}{l}\text { Process } \\
\text { Rank } 3 \\
\text { (abreviated) }\end{array}$ & $\begin{array}{l}\text { Process } \\
\text { Rank } 4 \\
\text { (abreviated) }\end{array}$ & $\begin{array}{l}\text { Identity } \\
\text { Process } \\
\text { Rank }\end{array}$ & $\begin{array}{l}\text { Priority } \\
\text { Process } \\
\text { Rank }\end{array}$ & $\begin{array}{l}\text { Back ground } \\
\text { Process } \\
\text { Rark }\end{array}$ & $\begin{array}{l}\text { Mandated } \\
\text { Process } \\
\text { Rank }\end{array}$ \\
\hline 59 & $1 \mathrm{H}$ & 3250.449 & $M$ & 1 & $\mathrm{~B}$ & $\mathrm{P}$ & 2 & 4 & 3 & 1 \\
\hline 60 & $2 \mathrm{M}$ & 5610.078 & B & $M$ & $\mathrm{P}$ & 1 & 4 & 3 & 1 & 2 \\
\hline 61 & $2 \mathrm{M}$ & 5220.499 & $M$ & B & $\mathrm{P}$ & 1 & 4 & 3 & 2 & 1 \\
\hline 62 & $2 \mathrm{M}$ & 2210.141 & $M$ & B & 1 & $\mathrm{P}$ & 3 & 4 & 2 & 1 \\
\hline 63 & $1 \mathrm{H}$ & 3260.036 & $B$ & $\mathrm{P}$ & 1 & $M$ & 3 & 2 & 1 & 4 \\
\hline 64 & $2 \mathrm{M}$ & 5110.005 & $\mathrm{~B}$ & $M$ & 1 & $\mathrm{P}$ & 3 & 4 & 1 & 2 \\
\hline 65 & $2 \mathrm{M}$ & 4430.006 & B & $M$ & $\mathrm{P}$ & 1 & 4 & 3 & 1 & 2 \\
\hline 66 & $2 \mathrm{M}$ & 3350.009 & 1 & B & $\mathrm{P}$ & $M$ & 1 & 3 & 2 & 4 \\
\hline 67 & $1 \mathrm{H}$ & 3340.131 & $\mathrm{P}$ & 1 & $M$ & B & 2 & 1 & 4 & 3 \\
\hline 68 & $2 \mathrm{M}$ & 5410.150 & $M$ & $\mathrm{P}$ & B & 1 & 4 & 2 & 3 & 1 \\
\hline 69 & & 3250.035 & $\mathrm{P}$ & $M$ & B & & 5 & 1 & 3 & 2 \\
\hline 71 & $1 \mathrm{H}$ & 3340.477 & B & 1 & $\mathrm{P}$ & $M$ & 2 & 3 & 1 & 4 \\
\hline 73 & $1 \mathrm{H}$ & 5220.417 & B & $M$ & $\mathrm{P}$ & 1 & 4 & 3 & 1 & 2 \\
\hline 74 & $1 \mathrm{H}$ & 8120.028 & $\mathrm{~B}$ & $M$ & $\mathrm{P}$ & 1 & 4 & 3 & 1 & 2 \\
\hline 75 & $2 \mathrm{M}$ & 2110.145 & $\mathrm{~B}$ & $M$ & & & 5 & 5 & 1 & 2 \\
\hline 76 & & 4410.025 & 1 & $\mathrm{P}$ & $M$ & B & 1 & 2 & 4 & 3 \\
\hline
\end{tabular}




\section{Appendix P: Financial Nonfinancial $\mathrm{H}_{2}$ Kruskal-Wallis Ranked Means}

by Clockspeed

\begin{tabular}{|c|c|c|c|c|c|c|c|c|}
\hline \begin{tabular}{|l} 
Resp \\
ID \\
\end{tabular} & $\begin{array}{l}1 \mathrm{H}, 2 \mathrm{HI} \\
3 \mathrm{~L} \\
\text { Raık }\end{array}$ & $\begin{array}{l}\text { Prodict } \\
\text { Clange } \\
(1,2,3)\end{array}$ & $\begin{array}{l}\text { Process } \\
\text { Clange }\end{array}$ & $\begin{array}{l}\text { Pert. } \\
\text { Meas I } \\
\text { Clalge }\end{array}$ & $\begin{array}{l}\text { F II al chal } \\
\text { Me a I I res }\end{array}$ & $\begin{array}{l}\text { Floascbl } \\
\text { lie as I res } \\
\text { Rauked w/ } \\
\text { tos }\end{array}$ & $\begin{array}{l}\text { Nol- } \\
\text { Fluaıc bI } \\
\text { Meası I s }\end{array}$ & $\begin{array}{l}\text { Nouth ar ckal } \\
\text { Me as I res } \\
\text { Rauked w/ } \\
\text { tes }\end{array}$ \\
\hline 6 & $1 \mathrm{H}$ & & 1 & 1 & 10 & 35.5 & 28 & 12 \\
\hline 8 & $1 \mathrm{H}$ & & 1 & 2 & 5 & 10.5 & 30 & 45 \\
\hline 10 & $1 \mathrm{H}$ & & 2 & 2 & 6 & 15.5 & 26 & 40 \\
\hline 11 & $1 \mathrm{H}$ & & 1 & 1 & 20 & 58 & 65 & 63 \\
\hline 14 & $1 \mathrm{H}$ & & 1 & 1 & 10 & 35.5 & 23 & 38.5 \\
\hline 23 & $1 \mathrm{H}$ & & 1 & 2 & 10 & 35.5 & 21 & 37 \\
\hline 24 & $1 \mathrm{H}$ & & 3 & 1 & 300 & 70 & 550 & 70 \\
\hline 29 & $1 \mathrm{H}$ & & 3 & 2 & 8 & 24 & 10 & 14 \\
\hline 30 & $1 \mathrm{H}$ & & 1 & 1 & 50 & 68.5 & 150 & 67 \\
\hline 31 & $1 \mathrm{H}$ & & 1 & 1 & 10 & 35.5 & 40 & 54 \\
\hline 35 & $1 \mathrm{H}$ & & 1 & 1 & 8 & 24 & 14 & 25.5 \\
\hline 35 & $1 \mathrm{H}$ & & 2 & 2 & 5 & 10.5 & 20 & 35 \\
\hline 37 & $1 \mathrm{H}$ & & 2 & 1 & 9 & 26.5 & 23 & 38.5 \\
\hline 39 & $1 \mathrm{H}$ & & 1 & 1 & 40 & 67 & 30 & 15 \\
\hline 40 & $1 \mathrm{H}$ & & 1 & 1 & 13 & 47.5 & 230 & 69 \\
\hline 42 & $1 \mathrm{H}$ & & 2 & 2 & 7 & 20 & 5 & 3 \\
\hline 45 & $1 \mathrm{H}$ & & 3 & 1 & 25 & 64 & 35 & 49.5 \\
\hline 18 & $1 \mathrm{H}$ & & 1 & 1 & 20 & 58 & 30 & 45 \\
\hline 49 & $1 \mathrm{H}$ & & 1 & 1 & 15 & 51.5 & 16 & 29.5 \\
\hline 52 & $1 \mathrm{H}$ & & 1 & 1 & 12 & 45 & 44 & 58.5 \\
\hline 53 & $1 \mathrm{H}$ & & 1 & 1 & 15 & 51.5 & 20 & 35 \\
\hline 59 & $1 \mathrm{H}$ & & 2 & 2 & 25 & 64 & 90 & 65 \\
\hline 63 & $1 \mathrm{H}$ & & 1 & 1 & 12 & 45 & 28 & 12 \\
\hline 67 & $1 \mathrm{H}$ & & 1 & 1 & 4 & 5 & 11 & 18.5 \\
\hline 71 & $1 \mathrm{H}$ & & 1 & 1 & 15 & 51.5 & 35 & 49.5 \\
\hline 73 & $1 \mathrm{H}$ & & 1 & 1 & 20 & 58 & 70 & 64 \\
\hline 74 & $1 \mathrm{H}$ & & 2 & 1 & 20 & 58 & 40 & 54 \\
\hline \multicolumn{5}{|c|}{ Hyb Peitome r Alverage Ralked the als } & \multicolumn{2}{|c|}{12.055556} & \multicolumn{2}{|c|}{44.3703704} \\
\hline 2 & 2 In & & 1 & 1 & 10 & 35.5 & 13 & 22 \\
\hline 3 & $2 n$ & & 1 & 1 & 4 & 5 & 14 & 25.5 \\
\hline & & & 1 & 1 & 20 & 58 & 60 & 62 \\
\hline 12 & & & 2 & 2 & 20 & 58 & 220 & 68 \\
\hline 13 & & & 1 & 1 & 10 & 35.5 & 10 & 14 \\
\hline 19 & & & 1 & 1 & 10 & 35.5 & 16 & 29.5 \\
\hline 22 & $2 \mathrm{ll}$ & & 3 & 2 & 7 & 20 & 11 & 18.5 \\
\hline 25 & & & 1 & 1 & 7 & 20 & 13 & 22 \\
\hline 32 & & & 2 & 2 & 10 & 35.5 & 28 & 42 \\
\hline 33 & & & 1 & 1 & 7 & 20 & 7 & 6 \\
\hline 38 & & & 1 & 1 & 4 & 5 & 10 & 14 \\
\hline 41 & & & 1 & 2 & 20 & 58 & 41 & 56 \\
\hline 13 & & & 1 & 2 & 10 & 35.5 & 35 & 49.5 \\
\hline 44 & & & 2 & 2 & 13 & 47.5 & 13 & 22 \\
\hline 55 & & & 1 & 1 & 10 & 35.5 & 14 & 25.5 \\
\hline 55 & 21.1 & & 1 & 1 & 5 & 10.5 & 18 & 31.5 \\
\hline 57 & & & 1 & 1 & 35 & 65 & 10 & 14 \\
\hline 60 & & & 1 & 1 & 10 & 35.5 & 11 & 18.5 \\
\hline 61 & & & 1 & 1 & 12 & 45 & 51 & 60 \\
\hline 62 & & & 3 & 2 & 6 & 15.5 & 37 & 52 \\
\hline 64 & & & 1 & 1 & 5 & 10.5 & 40 & 54 \\
\hline 65 & & & 1 & 1 & 10 & 35.5 & 19 & 33 \\
\hline 65 & & & 1 & 1 & 7 & 20 & 11 & 18.5 \\
\hline 68 & & & 3 & 1 & 15 & 51.5 & 20 & 35 \\
\hline 75 & & & 3 & 1 & 10 & 35.5 & 3 & 3 \\
\hline \multicolumn{5}{|c|}{ He dlum Pe nomner Ave age Rauked l.eaus } & & 33.2 & & 31.8 \\
\hline & $3 \mathrm{~L}$ & & 1 & 1 & 50 & 68.5 & 55 & 61 \\
\hline & $3 L$ & & 1 & 2 & 4 & 5 & 42 & 57 \\
\hline 9 & $3 \mathrm{~L}$ & & 1 & 1 & 20 & 58 & 35 & 49.5 \\
\hline 15 & & & 1 & & 14 & 49 & 18 & 31.5 \\
\hline 16 & & & & 1 & 9 & 26.5 & 15 & 28 \\
\hline 18 & & & 1 & 1 & 3 & 2 & 9 & 10.5 \\
\hline 20 & & & 1 & 1 & 10 & 35.5 & 14 & 25.5 \\
\hline 21 & & & 1 & 2 & 8 & 24 & 9 & 10.5 \\
\hline 25 & & & 2 & 3 & 6 & 15.5 & 8 & 8.5 \\
\hline 28 & & & 1 & 1 & 10 & 35.5 & 10 & 14 \\
\hline 34 & & & 3 & 3 & 5 & 10.5 & 7 & 0 \\
\hline 45 & & & & 2 & 2 & 1 & 8 & 8.5 \\
\hline 47 & & & 1 & 1 & 10 & 35.5 & 7 & 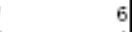 \\
\hline 51 & & & 3 & 3 & 5 & 10.5 & 0 & 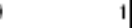 \\
\hline 54 & & & 2 & 2 & 6 & 15.5 & 6 & 5 \\
\hline 58 & & & 2 & 1 & 4 & 5 & 32 & 47 \\
\hline 69 & & & 1 & 1 & 25 & 64 & 44 & 58.5 \\
\hline 76 & & & 2 & 1 & 20 & 58 & 80 & 65 \\
\hline \multicolumn{5}{|c|}{ Lon Pe nome r Ave age Ravked the an s } & \multicolumn{2}{|r|}{28.851111} & \multicolumn{2}{|r|}{27.3333333} \\
\hline
\end{tabular}




\begin{tabular}{|c|c|c|c|c|c|c|c|c|c|c|}
\hline $\begin{array}{l}\mathrm{R} \in \mathrm{s}=\mathrm{p} \\
{[\mathrm{ID}}\end{array}$ & $\begin{array}{l}1 \mathrm{H}, 2 \mathrm{hl} \\
\partial \mathrm{L} \\
\text { Fenkt }\end{array}$ & $\begin{array}{l}\text { Produst } \\
\text { Change } \\
(1,2,3)\end{array}$ & $\begin{array}{l}\text { Financial } \\
\text { hule azures }\end{array}$ & $\begin{array}{l}\text { Financial } \\
\text { Measunes } \\
\text { Rianked wid } \\
\text { ties }\end{array}$ & $\begin{array}{l}\text { Non- } \\
\text { Financia } \\
\text { huleasures }\end{array}$ & $\begin{array}{l}\text { Monfinancial } \\
\text { Muleasures } \\
\text { Fanked wi } \\
\text { ties }\end{array}$ & $\begin{array}{l}\text { Financia } \\
\text { Masears } \\
\text { Ang }\end{array}$ & Count & & $\begin{array}{l}\text { Won- } \\
\text { inan cial } \\
\text { uleans } \\
\text { Aog }\end{array}$ \\
\hline$\overline{6}$ & $1 \mathrm{H}$ & 1 & 100 & 35.5 & 28 & 42 & & & & \\
\hline 8 & $1 \mathrm{H}$ & 1 & 5 & 10.5 & 30 & 46 & & & & \\
\hline 11 & $1 \mathrm{H}$ & 1 & 20 & 59 & 65 & 63 & & & & \\
\hline 14 & $1 \mathrm{H}$ & 1 & 100 & 35.5 & 20 & 385 & & & & \\
\hline 23 & $1 \mathrm{H}$ & $i$ & 10 & 35.5 & 21 & 37 & & & & \\
\hline 30 & $1 \mathrm{H}$ & 1 & 50 & 68.5 & 150 & 67 & & & & \\
\hline 31 & & 1 & 10 & 35.5 & 40 & 54 & & & & \\
\hline 35 & $1 \mathrm{H}$ & 1 & 9 & 24 & 14 & 25.5 & & & & \\
\hline 39 & $1 \mathrm{H}$ & 1 & 40 & 67 & 30 & 45 & & & & \\
\hline 40 & $1 \mathrm{H}$ & 1 & 13 & 47.5 & 230 & 60 & & & & \\
\hline 48 & $1 \mathrm{H}$ & 1 & 20 & 58 & 30 & 45 & & & & \\
\hline 49 & $1 \mathrm{H}$ & $i$ & 15 & 51.5 & 16 & 20.5 & & & & \\
\hline 52 & $1 \mathrm{H}$ & 1 & 12 & 45 & 44 & 58.5 & & & & \\
\hline 53 & $1 \mathrm{H}$ & 1 & 15 & 51.5 & 20 & 35 & & & & \\
\hline 63 & $1 \mathrm{H}$ & 1 & 12 & 45 & 28 & 42 & & & & \\
\hline 67 & $1 \mathrm{H}$ & 1 & 4 & 5 & 11 & 19.5 & & & & \\
\hline 71 & & 1 & 15 & 51.5 & 35 & 40.5 & & & & \\
\hline 73 & $1 \mathrm{H}$ & 1 & 20 & 50 & 70 & 64 & 435 & $1:$ & $\theta$ & 46 \\
\hline 2 & $2 \mathrm{hd}$ & 1 & 10 & 35.5 & 13 & 22 & & & & \\
\hline 3 & $2 \mathrm{w} 1$ & 1 & 4 & 5 & 14 & 25.5 & & & & \\
\hline 5 & $2 \mathrm{Mu}$ & 1 & 20 & 58 & 80 & 62 & & & & \\
\hline 13 & $2 \mathrm{ly}$ & $i$ & 10 & 35.5 & 10 & 14 & & & & \\
\hline 19 & $2 \mathrm{M}$ & 1 & 10 & 35.5 & 16 & 20.5 & & & & \\
\hline 26 & $2 \mathrm{l} 1$ & $i$ & 7 & 20 & 13 & 22 & & & & \\
\hline 33 & $2 \mathrm{w}$ & 1 & 7 & 20 & 7 & 6 & & & & \\
\hline 38 & $2 \mathrm{~W}$ & $i$ & 4 & 5 & 10 & 14 & & & & \\
\hline 41 & $2 \mathrm{hy}$ & $i$ & 20 & 58 & 41 & 56 & & & & \\
\hline 43 & $2 \mathrm{hi}$ & 1 & 10 & 35.5 & 35 & 40.5 & & & & \\
\hline 55 & $2 M$ & 1 & 10 & 35.5 & 14 & 25.5 & & & & \\
\hline 56 & $2 \mathrm{ht}$ & 1 & 5 & 10.5 & 18 & 31.5 & & & & \\
\hline 57 & $2 \mathrm{M}$ & 1 & 35 & 86 & 10 & 14 & & & & \\
\hline 60 & $2 \mathrm{hy}$ & 1 & 100 & 35.5 & 11 & 18.5 & & & & \\
\hline 61 & $2 \mathrm{~W}$ & $i$ & 12 & 45 & 51 & 60 & & & & \\
\hline 64 & $2 \mathrm{tw}$ & 1 & 5 & 10.5 & 40 & 54 & & & & \\
\hline 65 & $2 \mathrm{~W}$ & 1 & 10 & 35.5 & 19 & 33 & & & & \\
\hline 86 & $2 M$ & 1 & 7 & 20 & 11 & 18.5 & 31.47222 & $1:$ & 8 & 3086111 \\
\hline 4 & $3 \mathrm{~L}$ & 1 & 50 & 68.5 & 55 & 61 & & & & \\
\hline 7 & $3 \mathrm{~L}$ & 1 & 4 & 5 & 42 & 57 & & & & \\
\hline & $3 \mathrm{~L}$ & $i$ & 20 & 58 & 35 & 49.5 & & & & \\
\hline 15 & $3 \mathrm{~L}$ & 1 & 14 & 49 & 18 & 31.5 & & & & \\
\hline 18 & $3 \mathrm{~L}$ & 1 & 3 & 2 & 9 & 10.5 & & & & \\
\hline 20 & & 1 & 10 & 35.5 & 14 & 25.5 & & & & \\
\hline 21 & & $i$ & 8 & 24 & 9 & 10.5 & & & & \\
\hline 28 & $3 \mathrm{~L}$ & $i$ & 10 & 35.5 & 10 & 14 & & & & \\
\hline 47 & & 1 & 10 & 35.5 & 7 & 6 & & & & \\
\hline 69 & $3 \mathrm{~L}$ & 1 & 25 & 64 & 44 & 58.5 & 37.7 & 1t & 0 & 32.4 \\
\hline 10 & $1 \mathrm{H}$ & 2 & 6 & 15.5 & 26 & 40 & & & & \\
\hline 36 & $1 \mathrm{H}$ & 2 & 5 & 10.5 & 20 & 35 & & & & \\
\hline 37 & $1 \mathrm{H}$ & 2 & 9 & 26.5 & 20 & 39.5 & & & & \\
\hline 42 & $1 \mathrm{H}$ & 2 & 7 & 20 & 5 & 3 & & & & \\
\hline 59 & $1 \mathrm{H}$ & 2 & 25 & 64 & 90 & 66 & & & & \\
\hline 74 & & 2 & 20 & 58 & 40 & 54 & 32.41667 & & 6 & 39.41667 \\
\hline 12 & $2 \mathrm{w}$ & 2 & 20 & 58 & 220 & 68 & & & & \\
\hline 32 & $2 \mathrm{M}$ & 2 & 10 & 35.5 & 29 & 42 & & & & \\
\hline 44 & $2 \mathrm{M}$ & 2 & 13 & 47.5 & 13 & 22 & 47 & & 3 & 44 \\
\hline 25 & $3 \mathrm{~L}$ & 2 & 6 & 15.5 & 9 & 9.5 & & & & \\
\hline 54 & & 2 & 6 & 15.5 & 6 & 4 & & & & \\
\hline 58 & & 2 & 4 & 5 & 32 & 47 & & & & \\
\hline 76 & $3 \mathrm{~L}$ & 2 & 20 & 58 & 80 & 65 & 235 & & 4 & 31.125 \\
\hline 24 & $1 \mathrm{H}$ & 3 & 300 & 70 & 550 & 70 & & & & \\
\hline 29 & $1 \mathrm{H}$ & 3 & $\theta$ & 24 & 10 & 14 & & & & \\
\hline 45 & & 3 & 25 & 64 & 35 & 49.5 & 52.66667 & & 3 & 44.5 \\
\hline 22 & $2 M$ & 3 & 7 & 20 & 11 & 19.5 & & & & \\
\hline 62 & $2 \mathrm{w}$ & 3 & 6 & 15.5 & 37 & 52 & & & & \\
\hline 68 & $2 \mathrm{w}$ & 3 & 15 & 51.5 & 20 & 35 & & & & \\
\hline 75 & $2 \mathrm{~W}$ & 3 & 10 & 355 & 3 & 2 & 30.625 & & 4 & 26.875 \\
\hline 34 & & 3 & 5 & 10.5 & 7 & 6 & & & & \\
\hline 51 & $3 \mathrm{~L}$ & 3 & 5 & 10.5 & ô & 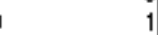 & 105 & & 2 & 35 \\
\hline 16 & $3 \mathrm{~L}$ & & $\bar{g}$ & 26.5 & 15 & 28 & & & & \\
\hline 46 & $3 \mathrm{~L}$ & & 2 & 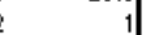 & 8 & 8.5 & & & & \\
\hline
\end{tabular}




\begin{tabular}{|c|c|c|c|c|c|c|c|c|c|c|c|}
\hline Resp & $\begin{array}{l}1 \mathrm{H}, 2 \mathrm{hW} \\
3 \mathrm{~L} \\
\text { Fenik }\end{array}$ & $\begin{array}{l}\text { Product } \\
\text { Change } \\
(1,2,3)\end{array}$ & $\begin{array}{l}\text { Prooess } \\
\text { Change }\end{array}$ & $\begin{array}{l}\text { Pert. } \\
\text { Wheasure } \\
\text { Change }\end{array}$ & $\begin{array}{l}\text { Financial } \\
\text { twe asures }\end{array}$ & $\begin{array}{l}\text { Financial } \\
\text { huleasunes } \\
\text { Fianked wi } \\
\text { ties }\end{array}$ & $\begin{array}{l}\text { Won- } \\
\text { Financia } \\
\text { Wheasures }\end{array}$ & $\begin{array}{l}\text { Nonfinancial } \\
\text { Whasures } \\
\text { Fenked wi } \\
\text { ties }\end{array}$ & $\begin{array}{l}\text { Financial } \\
\text { Wears: } \\
\text { Aug }\end{array}$ & Bount & $\begin{array}{l}\text { Pbn- } \\
\text { inancial } \\
\text { Whans } \\
\text { Ang }\end{array}$ \\
\hline$\overline{8}$ & $1 \mathrm{H}$ & & & 1 & 10 & 35.5 & 28 & 42 & & & \\
\hline 11 & $1 \mathrm{H}$ & & & 1 & 20 & 58 & 65 & 63 & & & \\
\hline 14 & $1 \mathrm{H}$ & & & 1 & 10 & 35.5 & 23 & 39.5 & & & \\
\hline 24 & $1 \mathrm{H}$ & & & 1 & 3000 & 70 & 560 & 70 & & & \\
\hline 30 & $1 \mathrm{H}$ & & & 1 & 50 & 60.5 & 150 & 67 & & & \\
\hline 31 & $1 \mathrm{H}$ & & & 1 & 100 & 35.5 & 40 & 54 & & & \\
\hline 35 & $1 \mathrm{H}$ & & & 1 & 8 & 24 & 14 & 25.5 & & & \\
\hline 37 & $1 \mathrm{H}$ & & & 1 & 9 & 26.5 & 23 & 38.5 & & & \\
\hline 39 & $1 \mathrm{H}$ & & & 1 & 40 & 67 & 30 & 45 & & & \\
\hline 40 & $1 \mathrm{H}$ & & & 1 & 13 & 47.5 & 230 & 69 & & & \\
\hline 45 & $1 \mathrm{H}$ & & & 1 & 25 & 64 & 35 & 49.5 & & & \\
\hline 48 & $1 \mathrm{H}$ & & & 1 & 20 & 58 & 30 & 45 & & & \\
\hline 49 & $1 \mathrm{H}$ & & & 1 & 15 & 51.5 & 16 & 29.5 & & & \\
\hline 52 & $1 \mathrm{H}$ & & & 1 & 12 & 45 & 44 & 58.5 & & & \\
\hline 50 & $1 \mathrm{H}$ & & & 1 & 15 & 51.5 & 20 & 35 & & & \\
\hline 63 & $1 \mathrm{H}$ & & & 1 & 12 & 45 & 28 & 42 & & & \\
\hline 67 & $1 \mathrm{H}$ & & & 1 & 4 & 5 & 11 & 18.5 & & & \\
\hline 71 & $1 \mathrm{H}$ & & & 1 & 15 & 51.5 & 35 & 49.5 & & & \\
\hline 73 & $1 \mathrm{H}$ & & & 1 & 20 & 58 & 70 & 64 & & & \\
\hline 74 & & & & 1 & 20 & 58 & 40 & 54 & 47.775 & 2 & 47. \\
\hline 22 & $2 \mathrm{M}$ & & & 1 & 10 & 35.5 & 13 & 22 & & & \\
\hline 32 & $2 \mathrm{M}$ & & & 1 & 4 & 5 & 14 & 25.5 & & & \\
\hline 5 & $2 \mathrm{~m}$ & & & 1 & 20 & 58 & 60 & 62 & & & \\
\hline 132 & $2 \mathrm{M}$ & & & 1 & 10 & 35.5 & 10 & 14 & & & \\
\hline $19:$ & $2 M$ & & & 1 & 10 & 35.5 & 16 & 29.5 & & & \\
\hline $26:$ & $2 \mathrm{~W}$ & & & 1 & 7 & 20 & 13 & 22 & & & \\
\hline 302 & $2 \mathrm{M}$ & & & 1 & 7 & 20 & 7 & 6 & & & \\
\hline 382 & $2 \mathrm{M}$ & & & 1 & 4 & 5 & 10 & 14 & & & \\
\hline 55 & $2 \mathrm{MI}$ & & & 1 & 100 & 35.5 & 14 & 25.5 & & & \\
\hline 562 & $2 \mathrm{w}$ & & & 1 & 5 & 10.5 & 18 & 31.5 & & & \\
\hline 57 & $2 \mathrm{M}$ & & & 1 & 35 & 66 & 10 & 14 & & & \\
\hline 602 & $2 \mathrm{M}$ & & & 1 & 10 & 35.5 & 11 & 18.5 & & & \\
\hline 612 & $2 \mathrm{M}$ & & & 1 & 12 & 45 & 51 & 60 & & & \\
\hline 642 & $2 \mathrm{~m}$ & & & 1 & 5 & 10.5 & 40 & 54 & & & \\
\hline 65 & $2 \mathrm{M}$ & & & 1 & 10 & 35.5 & 19 & 33 & & & \\
\hline 660 & $2 \mathrm{~m}$ & & & 1 & 7 & 20 & 11 & 18.5 & & & \\
\hline $68:$ & $2 \mathrm{~W}$ & & & 1 & 15 & 51.5 & 20 & 35 & & & \\
\hline 75 & $2 \mathrm{M}$ & & & 1 & 10 & 35.5 & 3 & 2 & 31.11111 & 1 & $9 \quad 270555$ \\
\hline 43 & $3 \mathrm{~L}$ & & & 1 & 50 & 68.5 & 55 & 61 & & & \\
\hline $9:$ & $3 L$ & & & 1 & 20 & 58 & 35 & 49.5 & & & \\
\hline $16:$ & $3 \mathrm{~L}$ & & & 1 & 9 & 26.5 & 15 & 28 & & & \\
\hline $18:$ & $3 \mathrm{~L}$ & & & 1 & 3 & 2 & 9 & 10.5 & & & \\
\hline $20: 3$ & $3 \mathrm{~L}$ & & & 1 & 10 & 35.5 & 14 & 25.5 & & & \\
\hline 283 & $3 \mathrm{~L}$ & & & 1 & 10 & 35.5 & 10 & 14 & & & \\
\hline 47 & $3 \mathrm{~L}$ & & & 1 & 10 & 35.5 & 7 & 6 & & & \\
\hline $58:$ & $3 \mathrm{~L}$ & & & 1 & 4 & 5 & 32 & 47 & & & \\
\hline $69:$ & $3 \mathrm{~L}$ & & & 1 & 25 & 64 & 44 & 58.5 & & & \\
\hline $76:$ & $3 \mathrm{~L}$ & & & 1 & 20 & 58 & 80 & 65 & 3885 & 16 & 364 \\
\hline 9 & $1 \mathrm{HH}$ & & & 2 & 5 & 10.5 & 30 & 45 & & & \\
\hline 10 & $1 \mathrm{H}$ & & & 2 & 6 & 15.5 & 26 & 40 & & & \\
\hline 23 & $1 \mathrm{H}$ & & & 2 & 100 & 35.5 & 21 & 37 & & & \\
\hline 29 & $1 \mathrm{H}$ & & & 2 & 8 & 24 & 10 & 14 & & & \\
\hline 36 & $1 \mathrm{H}$ & & & 2 & 5 & 10.5 & 20 & 35 & & & \\
\hline 42 & $1 \mathrm{H}$ & & & 2 & 7 & 20 & 5 & 3 & & & \\
\hline 59 & $1 \mathrm{H}$ & & & 2 & 25 & 64 & 90 & 66 & 25.71429 & & 7342857 \\
\hline 122 & $2 \mathrm{Wt}$ & & & 2 & 20 & 58 & 220 & 68 & & & \\
\hline 22 & $2 \mathrm{M}$ & & & 2 & 7 & 20 & 11 & 18.5 & & & \\
\hline 32 & $2 \mathrm{M}$ & & & 2 & $10 \overline{0}$ & 35.5 & 28 & 42 & & & \\
\hline $41:$ & $2 \mathrm{M}$ & & & 2 & 20 & 58 & 41 & 56 & & & \\
\hline 432 & $2 \mathrm{M}$ & & & 2 & 10 & 35.5 & 35 & 49.5 & & & \\
\hline 442 & & & & 2 & 13 & 47.5 & 13 & 22 & & & \\
\hline 622 & $2 \mathrm{M}$ & & & 2 & 8 & 15.5 & 37 & 52 & 39.57149 & & 7 \\
\hline $7:$ & $3 \mathrm{~L}$ & & & 2 & 4 & 5 & 42 & 57 & & & \\
\hline $21:$ & $3 \mathrm{~L}$ & & & 2 & 9 & 24 & 9 & 10.5 & & & \\
\hline $46:$ & & & & 2 & 2 & 1 & 8 & 8.5 & & & \\
\hline 543 & & & & 2 & 6 & 15.5 & 6 & 4 & 11375 & & 4 \\
\hline $25:$ & & & & 9 & 6 & 15.5 & 8 & 8.5 & & & \\
\hline $34: 3$ & & & & 9 & 5 & 10.5 & 7 & 6 & & & \\
\hline $51:$ & & & & 3 & 5 & 10.5 & 0 & 1 & 12.16667 & & 35.16666 \\
\hline 153 & $3 \mathrm{~L}$ & & & & 14 & 49 & 18 & 31.5 & & & \\
\hline
\end{tabular}




\begin{tabular}{|c|c|c|c|c|c|c|c|c|c|c|c|c|}
\hline $\begin{array}{l}\text { Fisp } \\
\text { [L] }\end{array}$ & $\begin{array}{l}1 \mathrm{H}, 2 \mathrm{MI} \\
3 \mathrm{~L} \\
\text { Fenalk }\end{array}$ & $\begin{array}{l}\text { Product } \\
\text { Change } \\
(1,2,3)\end{array}$ & $\begin{array}{l}\text { Prowes } \\
\text { Change }\end{array}$ & $\begin{array}{l}\text { Perf. } \\
\text { Measure } \\
\text { Change }\end{array}$ & $\begin{array}{l}\text { Financial } \\
\text { Wheagures }\end{array}$ & $\begin{array}{l}\text { Financial } \\
\text { buleasures } \\
\text { Ranked wi } \\
\text { ties }\end{array}$ & $\begin{array}{l}\text { Won- } \\
\text { Financiz } \\
\text { hileagures }\end{array}$ & $\begin{array}{l}\text { Wonfinancial } \\
\text { heasures } \\
\text { Fennked wi' } \\
\text { ties }\end{array}$ & $\begin{array}{l}\text { Financial } \\
\text { heare } \\
\text { Aog }\end{array}$ & Count & & $\begin{array}{l}\text { Pon- } \\
\text { inancial } \\
\text { Means } \\
\text { Ang }\end{array}$ \\
\hline & $1 \mathrm{H}$ & & & 1 & $\overline{10 \overline{1}}$ & 35.5 & 28 & 42 & & & & \\
\hline 8 & $1 \mathrm{H}$ & & & 1 & 5 & 10.5 & 30 & 45 & & & & \\
\hline 11 & $1 \mathrm{H}$ & & & 1 & 20 & 58 & 65 & 63 & & & & \\
\hline 24 & $1 \mathrm{H}$ & & & 1 & 300 & 70 & 550 & 70 & & & & \\
\hline 30 & $1 \mathrm{H}$ & & & 1 & 50 & 68.5 & 150 & 67 & & & & \\
\hline 31 & $1 \mathrm{H}$ & & & 1 & 100 & 35.5 & 40 & 54 & & & & \\
\hline 30 & $1 \mathrm{H}$ & & & 1 & 40 & 67 & 30 & 45 & & & & \\
\hline 40 & $1 \mathrm{H}$ & & & 1 & 13 & 47.5 & 230 & 69 & & & & \\
\hline 52 & $1 \mathrm{H}$ & & & 1 & 12 & 45 & 44 & 58.5 & & & & \\
\hline 50 & $1 \mathrm{H}$ & & & 1 & 25 & 64 & 90 & 66 & & & & \\
\hline 67 & $1 \mathrm{H}$ & & & 1 & 4 & 5 & 11 & 18.5 & & & & \\
\hline 73 & $1 \mathrm{H}$ & & & 1 & 20 & 58 & 70 & 64 & 47.04167 & & 12 & 55.16667 \\
\hline 2 & 2 wi & & & 1 & 10 & 35.5 & 13 & 22 & & & & \\
\hline & $2 \mathrm{w}$ & & & 1 & 4 & 5 & 14 & 25.5 & & & & \\
\hline 12 & 2 w & & & 1 & 20 & 58 & 220 & 68 & & & & \\
\hline 13 & $2 \mathrm{hw}$ & & & 1 & 10 & 35.5 & 10 & 14 & & & & \\
\hline 19 & $2 \mathrm{hi}$ & & & 1 & 10 & 35.5 & 16 & 29.5 & & & & \\
\hline 33 & $2 \mathrm{hil}$ & & & 1 & 7 & 20 & 7 & 6 & & & & \\
\hline 44 & $2 \mathrm{w} 1$ & & & 1 & 13 & 47.5 & 13 & 22 & & & & \\
\hline 55 & & & & 1 & 10 & 35.5 & 14 & 25.5 & & & & \\
\hline 57 & & & & 1 & 35 & 60 & 10 & 14 & & & & \\
\hline 61 & & & & 1 & 12 & 45 & 51 & 60 & & & & \\
\hline 62 & & & & 1 & 6 & 15.5 & 37 & 52 & & & & \\
\hline 64 & & & & 1 & 5 & 10.5 & 40 & 54 & & & & \\
\hline 65 & $2 \mathrm{Wu}$ & & & 1 & 10 & 35.5 & 19 & 33 & & & & \\
\hline 69 & & & & 1 & 15 & 51.5 & 20 & 35 & 35.46429 & & 14 & 3299286 \\
\hline 4 & $3 \mathrm{~L}$ & & & 1 & 50 & 68.5 & 55 & 61 & & & & \\
\hline 7 & $3 \mathrm{~L}$ & & & 1 & 4 & 5 & 42 & 57 & & & & \\
\hline 18 & $3 \mathrm{~L}$ & & & 1 & 3 & 2 & 9 & 10.5 & & & & \\
\hline 25 & $3 \mathrm{~L}$ & & & 1 & 6 & 15.5 & 8 & 8.5 & & & & \\
\hline 60 & $3 \mathrm{~L}$ & & & 1 & 25 & 64 & 44 & 58.5 & & & & \\
\hline 76 & & & & 1 & 20 & 58 & 80 & 65 & 355 & & 6 & 4341667 \\
\hline 10 & & & & 2 & 6 & 15.5 & 26 & 40 & & & & \\
\hline 14 & $1 \mathrm{H}$ & & & 2 & 10 & 35.5 & 23 & 38.5 & & & & \\
\hline 35 & $1 \mathrm{H}$ & & & 2 & 8 & 24 & 14 & 25.5 & & & & \\
\hline 36 & $1 \mathrm{H}$ & & & $\bar{z}$ & 5 & 10.5 & 20 & 35 & & & & \\
\hline 37 & $1 \mathrm{H}$ & & & 2 & 9 & 26.5 & 23 & 38.5 & & & & \\
\hline 42 & $1 \mathrm{H}$ & & & 2 & 7 & 20 & 5 & 3 & & & & \\
\hline 45 & $1 \mathrm{H}$ & & & 2 & 25 & 64 & 35 & 49.5 & & & & \\
\hline 48 & $1 \mathrm{H}$ & & & 2 & 20 & 58 & 30 & 45 & & & & \\
\hline 49 & $1 \mathrm{H}$ & & & 2 & 15 & 51.5 & 16 & 29.5 & & & & \\
\hline 63 & $1 \mathrm{H}$ & & & $\overline{2}$ & 12 & 45 & 28 & 42 & & & & \\
\hline 74 & & & & 2 & 20 & 58 & 40 & 54 & 37.13656 & & 11 & 36.40009 \\
\hline 2 & & & & 2 & 7 & 20 & 11 & 18.5 & & & & \\
\hline 26 & 2 tw & & & 2 & 7 & 20 & 13 & 22 & & & & \\
\hline 32 & & & & 2 & 10 & 35.5 & 28 & 42 & & & & \\
\hline 41 & $2 \mathrm{w}$ & & & 2 & 20 & 58 & 41 & 56 & & & & \\
\hline 43 & $2 \mathrm{M}$ & & & 2 & 10 & 35.5 & 35 & 49.5 & & & & \\
\hline 56 & $2 \mathrm{hw}$ & & & 2 & 5 & 10.5 & 18 & 31.5 & & & & \\
\hline 80 & $2 \mathrm{M}$ & & & 2 & 10 & 35.5 & 11 & 18.5 & 30.71429 & & 7 & 34 \\
\hline$\overline{9}$ & $3 \mathrm{~L}$ & & & 2 & 20 & 58 & 35 & 49.5 & & & & \\
\hline 16 & & & & 2 & 9 & 26.5 & 15 & 28 & & & & \\
\hline 20 & & & & 2 & 10 & 35.5 & 14 & 25.5 & & & & \\
\hline 21 & & & & 2 & 8 & 24 & 9 & 10.5 & & & & \\
\hline 28 & & & & 2 & 10 & 35.5 & 10 & 14 & & & & \\
\hline 47 & $3 \mathrm{~L}$ & & & 2 & 10 & 35.5 & 7 & 6 & & & & \\
\hline 51 & & & & $\bar{z}$ & 5 & 10.5 & 0 & 1 & & & & \\
\hline 54 & & & & 2 & 6 & 15.5 & 6 & 4 & & & & \\
\hline 56 & & & & 2 & 4 & 5 & 32 & 47 & 27330 & & $g$ & 2061111 \\
\hline 23 & & & & 3 & $1 \overline{0}$ & 35.5 & 21 & 37 & 355 & & 1 & 37 \\
\hline 5 & $2 \mathrm{w}$ & & & 3 & 20 & 58 & 60 & 62 & & & & \\
\hline 38 & & & & 3 & 4 & 5 & 10 & 14 & & & & \\
\hline 75 & $2 \mathrm{w}$ & & & 3 & 10 & 35.5 & 3 & 2 & 32.8930 & & 3 & 26 \\
\hline 34 & & & & 3 & 5 & 100.5 & 7 & 6 & 1005 & & 1 & $\overline{6}$ \\
\hline 20 & & & & & 8 & 24 & 10 & 14 & & & & \\
\hline 53 & $1 \mathrm{H}$ & & & & 15 & 51.5 & 20 & 35 & & & & \\
\hline 71 & & & & & 15 & 51.5 & 35 & 49.5 & & & & \\
\hline 66 & 2 w & & & & 7 & 20 & 11 & 18.5 & & & & \\
\hline 15 & & & & & 14 & 40 & 18 & 31.5 & & & & \\
\hline 46 & $3 \mathrm{~L}$ & & & & 2 & 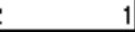 & 8 & 8.5 & & & & \\
\hline
\end{tabular}




\section{Appendix Q: $\mathrm{H}_{3}$ Cramer's Phi}
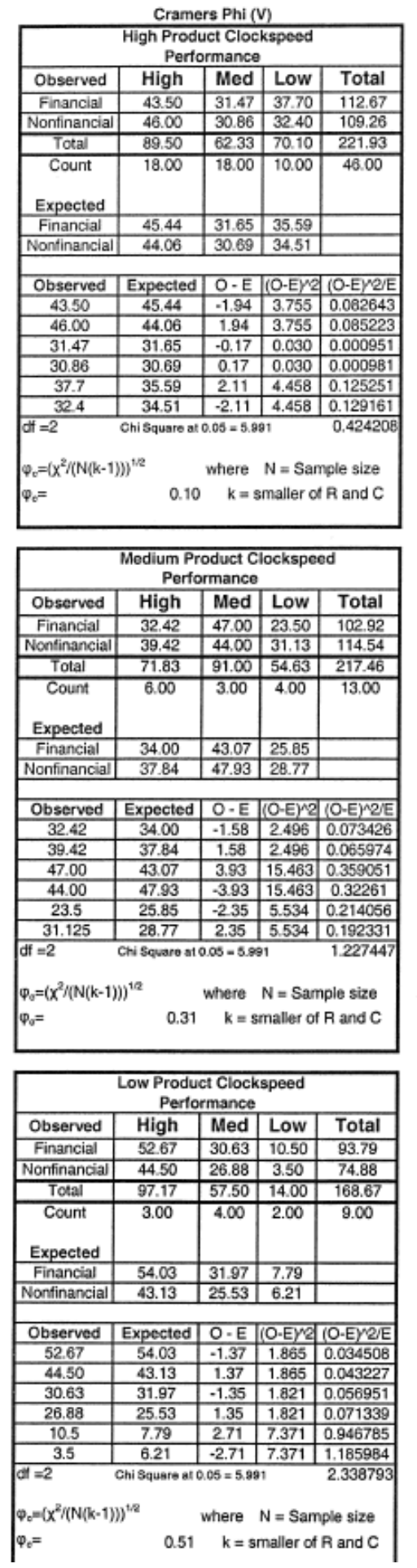
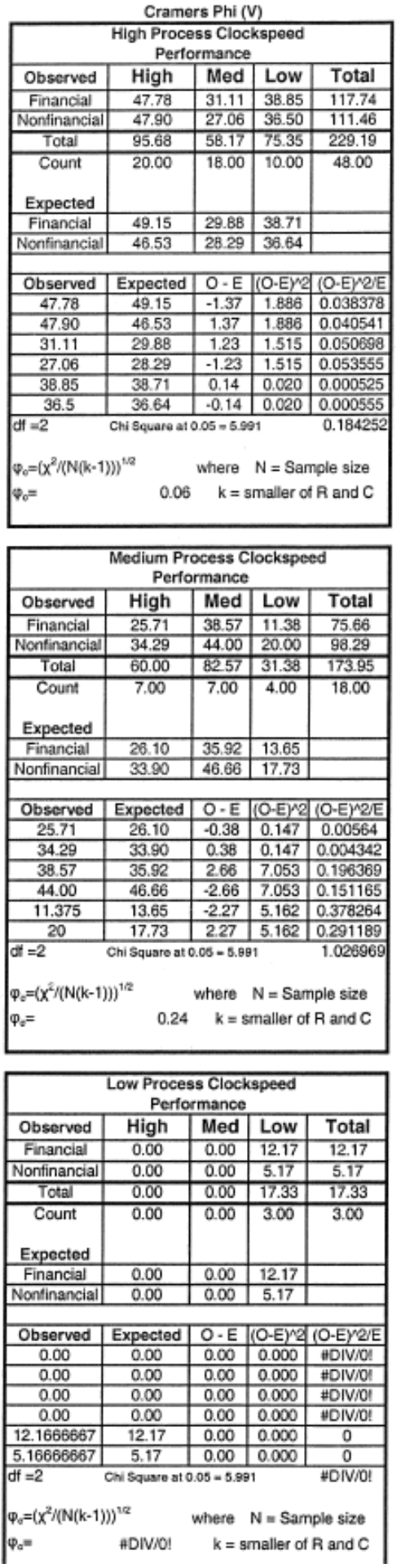

Cramers Phi (V)

\begin{tabular}{|c|c|c|c|c|}
\hline \multicolumn{5}{|c|}{$\begin{array}{l}\text { High Measurment System Clockspeed } \\
\text { Performance }\end{array}$} \\
\hline Observed & High & Med & Low & Total \\
\hline Financial & 47.04 & 35.46 & 35.50 & 118.01 \\
\hline Nonfinancial & 55.17 & 32.89 & 43.42 & 131.48 \\
\hline Total & 102.21 & 68.38 & 78.92 & 249.48 \\
\hline $\begin{array}{c}\text { Count } \\
\text { Expected }\end{array}$ & 12.00 & 14.00 & 6.00 & 32.00 \\
\hline Financial & 48.34 & 32.33 & 37.33 & \\
\hline Nonfinancial & 53.86 & 36.02 & 41.59 & \\
\hline Observed & Expected & $\mathrm{O} \cdot \mathrm{E}$ & O-E) & (O-EY) \\
\hline 47.04 & 48.34 & -1.30 & 1.698 & 0.035132 \\
\hline 55.17 & 53.86 & 1.30 & 1.698 & 0.031532 \\
\hline 35.46 & 32.33 & 3.13 & 9.804 & \begin{tabular}{|c|}
0.303214 \\
\end{tabular} \\
\hline 32.89 & 36.02 & -3.13 & 9.804 & 0.272148 \\
\hline 35.5 & 37.33 & -1.83 & 3.341 & \begin{tabular}{|l|}
0.089507 \\
\end{tabular} \\
\hline 43.4166667 & 41.59 & 1.83 & 3.341 & 0.080337 \\
\hline \multicolumn{5}{|l|}{$\mathrm{df}=2$} \\
\hline \multicolumn{5}{|c|}{$\varphi_{e}=\left(x^{2} /(N(k-1))\right)^{1 / 2}$} \\
\hline$\varphi_{c}=$ & 0.16 & $k=s$ & maller of & $\mathrm{R}$ and $\mathrm{C}$ \\
\hline
\end{tabular}

\begin{tabular}{|c|c|c|c|c|}
\hline \multicolumn{5}{|c|}{$\begin{array}{l}\text { Medium Measurment System Clockspeed } \\
\text { Performance }\end{array}$} \\
\hline Observed & High & Med & Low & Total \\
\hline Financial & 37,14 & 30.71 & 27.33 & 95.18 \\
\hline Nonfinancial & 36.41 & 34.00 & 20.61 & 91.02 \\
\hline Tolal & 73.55 & 64.71 & 47.94 & 186.20 \\
\hline Count & 11.00 & 7.00 & 9.00 & 27.00 \\
\hline \multicolumn{5}{|l|}{ Expected } \\
\hline Financial & 37.60 & 33.08 & 24.51 & \\
\hline Nonfinancial & 35.95 & 31.63 & 23.44 & \\
\hline Observed & Expected & $O=E$ & TO-EYQ & (O-E) $2 / E$ \\
\hline 37.14 & 37.60 & -0.46 & 0.210 & 0.005595 \\
\hline 36.41 & 35.95 & 0.46 & 0.210 & 0.005851 \\
\hline 30.71 & 3308 & -2.37 & 5.600 & 0.169279 \\
\hline 34.00 & 31.63 & 2.37 & 5.600 & 0.177023 \\
\hline 27.3333333 & 24.51 & 2.83 & 7.981 & 0.325644 \\
\hline \begin{tabular}{|l|}
20.6111111 \\
\end{tabular} & 23.44 & -2.83 & 7.981 & 0.34054 \\
\hline \multicolumn{5}{|c|}{ off $=2 \quad$ Chi square at $0.05=5.991 \quad 1.023934$} \\
\hline \multicolumn{5}{|c|}{$\varphi_{\mathrm{z}}=\left(\mathrm{X}^{2} /(\mathrm{N}(\mathrm{k}-1))\right)^{1 / 2}$} \\
\hline$\varphi_{\mathrm{g}}=$ & 0.19 & $k=$ & smaller o & $R$ and $C$ \\
\hline
\end{tabular}

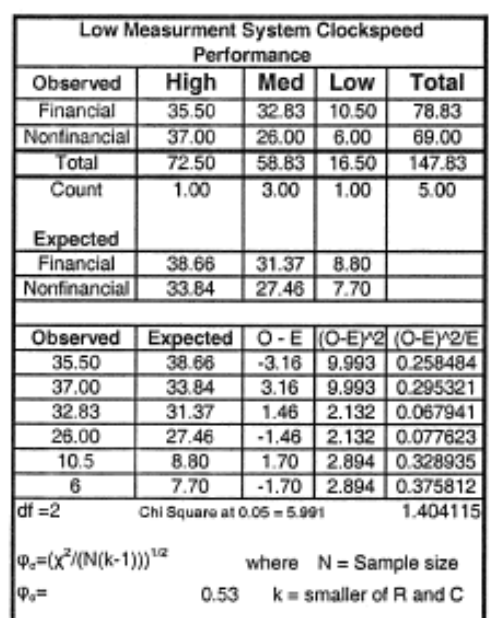




\section{Appendix R: Number of Balanced Scorecard Categories Used}

\begin{tabular}{|c|c|c|c|c|c|c|}
\hline $1 \mathrm{H}, 2 \mathrm{M}$ & & & Internal & Employee & & \\
\hline $\operatorname{Resp}, 3 \mathrm{~L}$ & & Financial & Operating & Related & Customer & $\mathrm{BSC}$ \\
\hline ID Rank & Company & Measures & Measures & Measures & Measures & Used \\
\hline $22 \mathrm{M}$ & 3360.048 & 10 & 5 & 5 & 3 & 4 \\
\hline $32 \mathrm{M}$ & 4230.135 & 4 & 7 & 2 & 5 & 4 \\
\hline $43 \mathrm{~L}$ & 7220.054 & 50 & 20 & 15 & 20 & 4 \\
\hline $52 \mathrm{M}$ & 4230.135 & 20 & 30 & 5 & 25 & 4 \\
\hline $61 \mathrm{H}$ & 5250.475 & 10 & 12 & 6 & 10 & 4 \\
\hline $73 \mathrm{~L}$ & 7130.039 & 4 & 20 & 2 & 20 & 4 \\
\hline $81 \mathrm{H}$ & 8120.006 & 5 & 20 & 5 & 5 & 4 \\
\hline $93 \mathrm{~L}$ & 5410.052 & 20 & 20 & 5 & 10 & 4 \\
\hline $101 \mathrm{H}$ & 5410.352 & 6 & 8 & 10 & 8 & 4 \\
\hline $111 \mathrm{H}$ & 3250.303 & 20 & 30 & 15 & 20 & 4 \\
\hline $122 \mathrm{~m}$ & 2210.105 & 20 & 150 & 20 & 50 & 4 \\
\hline $132 \mathrm{M}$ & 2370.001 & 10 & 3 & 4 & 3 & 4 \\
\hline $141 \mathrm{H}$ & 3110.038 & 10 & 10 & 5 & 8 & 4 \\
\hline $153 \mathrm{~L}$ & 3250.153 & 14 & 15 & 0 & 3 & 3 \\
\hline $163 \mathrm{~L}$ & 3110.058 & 9 & 8 & 4 & 3 & 4 \\
\hline $183 \mathrm{~L}$ & 3110.084 & 3 & 3 & 2 & 4 & 4 \\
\hline $192 \mathrm{M}$ & 5110.159 & 10 & 5 & 3 & 8 & 4 \\
\hline $203 \mathrm{~L}$ & 5220.625 & 10 & 2 & 5 & 7 & 4 \\
\hline $213 \mathrm{~L}$ & 5220.315 & 8 & 2 & 3 & 4 & 4 \\
\hline $222 \mathrm{~m}$ & 5620.030 & 7 & 2 & 5 & 4 & 4 \\
\hline $231 \mathrm{H}$ & 5220.349 & 10 & 10 & 7 & 4 & 4 \\
\hline $241 \mathrm{H}$ & 2210.101 & 300 & 400 & 50 & 100 & 4 \\
\hline $253 \mathrm{~L}$ & 3120.033 & 6 & 5 & 0 & 3 & 3 \\
\hline $26 \quad 2 \mathrm{M}$ & 3340.439 & 7 & 10 & 0 & 3 & 3 \\
\hline $283 \mathrm{~L}$ & 3330.130 & 10 & 10 & 0 & 0 & 2 \\
\hline $291 \mathrm{H}$ & 5330.016 & 8 & 5 & 3 & 2 & 4 \\
\hline $301 \mathrm{H}$ & 5220.319 & 50 & 100 & 25 & 25 & 4 \\
\hline $311 \mathrm{H}$ & 3330.070 & 10 & 20 & 10 & 10 & 4 \\
\hline $322 \mathrm{~m}$ & 3340.499 & 10 & 15 & 5 & 8 & 4 \\
\hline $332 \mathrm{~m}$ & 3340.601 & 7 & 3 & 2 & 2 & 4 \\
\hline $343 \mathrm{~L}$ & 2210.049 & 5 & 5 & 1 & 1 & 4 \\
\hline $351 \mathrm{H}$ & 5220.359 & 8 & 5 & 5 & 4 & 4 \\
\hline $361 \mathrm{H}$ & 5240.074 & 5 & 15 & 2 & 3 & 4 \\
\hline $371 \mathrm{H}$ & 5250.791 & 9 & 12 & 4 & 7 & 4 \\
\hline $382 \mathrm{~m}$ & 5220.337 & 4 & 5 & 3 & 2 & 4 \\
\hline $391 \mathrm{H}$ & 5220.135 & 40 & 10 & 10 & 10 & 4 \\
\hline $401 \mathrm{H}$ & 3360.074 & 13 & 200 & 10 & 20 & 4 \\
\hline $412 \mathrm{M}$ & 3390.087 & 20 & 25 & 6 & 10 & 4 \\
\hline $42 \mathrm{1H}$ & 3340.421 & 7 & 3 & 0 & 2 & 3 \\
\hline $432 \mathrm{~m}$ & 5150.009 & 10 & 20 & 5 & 10 & 4 \\
\hline $442 \mathrm{~m}$ & 3250.277 & 13 & 8 & 4 & 1 & 4 \\
\hline $451 \mathrm{H}$ & 3220.026 & 25 & 15 & 15 & 5 & 4 \\
\hline $463 \mathrm{~L}$ & 2370.027 & 2 & 4 & 2 & 2 & 4 \\
\hline $473 \mathrm{~L}$ & 5410.018 & 10 & 4 & 2 & 1 & 4 \\
\hline $481 \mathrm{H}$ & 5220.199 & 20 & 20 & 5 & 5 & 4 \\
\hline $491 \mathrm{H}$ & 5220.679 & 15 & 10 & 3 & 3 & 4 \\
\hline $513 \mathrm{~L}$ & 2110.060 & 5 & 0 & 0 & 0 & 1 \\
\hline $521 \mathrm{H}$ & 3340.191 & 12 & 24 & 10 & 10 & 4 \\
\hline $531 \mathrm{H}$ & 5220.233 & 15 & 10 & 2 & 8 & 4 \\
\hline
\end{tabular}




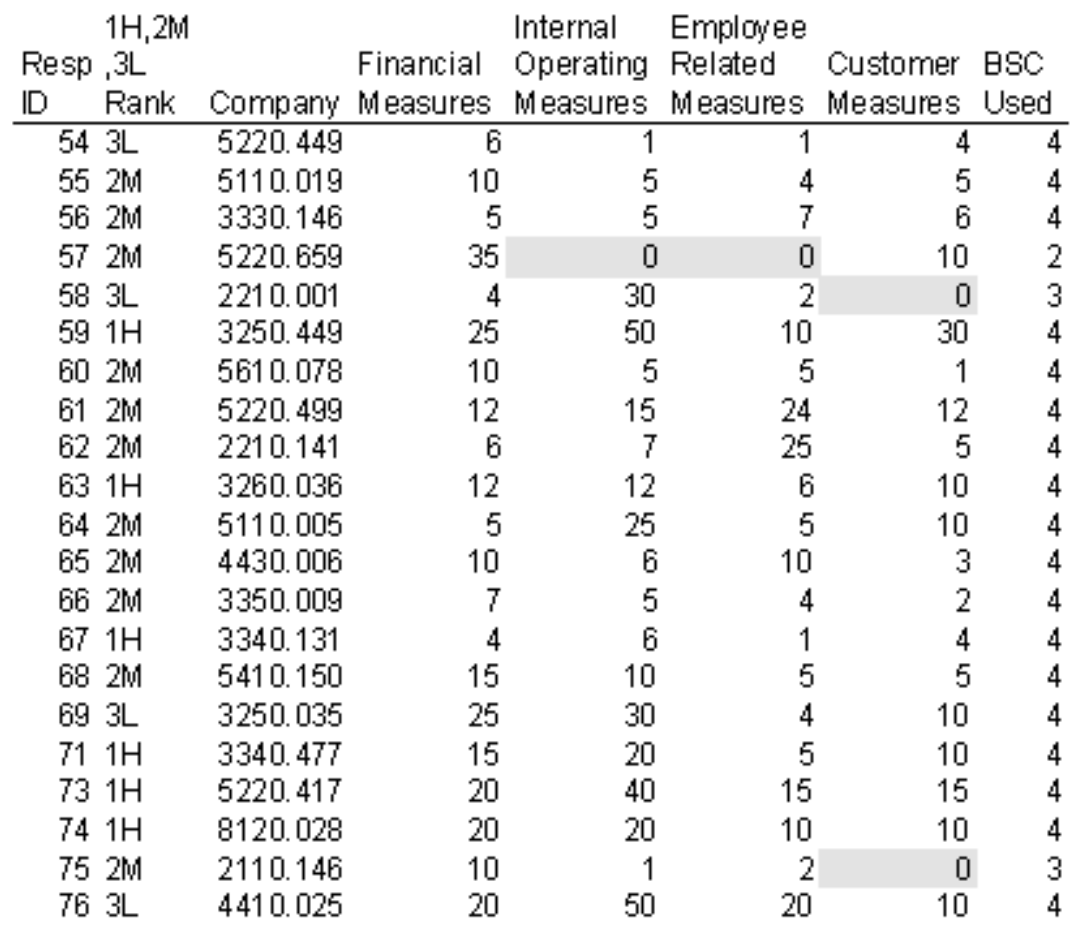




\section{Appendix S: Dissertation Survey}

\section{Portland State}

U N I VER S I T Y

\section{Dissertation Survey}

consent Form

Web-Based Survey

\section{Performance Measures for Managerial Decision Making: Performance Measurement Synergies in Multi-Attribute Performance Measurement Systems}

You are invited to participate in a research study conducted by Robert A. Fowke from Portland State University, Systems Science: Business Administration. The researcher hopes to learn the impact of use of different performance measures, varied feedback lags, and distribution of performance measurement information on business performance, in partial

If you decide to participate, you will be asked to complete the following questionnaire regarding performance measurement criteria and processes in your company/department.

Any information that is obtained in connection with this study and that can be linked to you or identify you will be kept confidential. This information will be kept confidential by Any information that is obtained in connection with this study and that can be linked to you or identify you will be kept confidential. This information will
coding of companies and participants (Company $x \times x x$, Participant $1-x$ ). Each participant has the option to receive results of the study upon request.

Your participation is voluntary. You do not have to take part in this study, and it will not affect your relationship with your company or Portland State University. You may also withdraw from this study at any time without affecting your relationship with your company or Portland State University.

If you have concerns or problems about your participation in this study or your rights as a research subject, please contact the Human Subjects Research Review Committee, Office of Research and Sponsored Projects, 600 Unitus Bldg., Portland State University, (503) 725-4288 / 1-877-480-4400.

If you have questions about the study itself, contact Robert Fowke at Portland State University, c/o Dr. Beverly Fuller, SBA.

Clicking the submit button at the end of the survey indicates that you have read and understand the above information and agree to take part in this study. Please understand that you may withdraw your consent at any time without penalty, and that, by not continuing, you are not waiving any legal claims, rights or remedies. The researcher will provide you with a copy of this form for your own records upon request.

Results of the study will be available to participants upon request.

1) Please enter your company code.

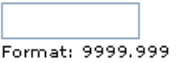

2) How frequently does your product change/improve? (check most appropriate)

$$
O<3 \text { years } O 3-10 \text { years } O>10 \text { years } O \text { Don't know }
$$

3) How frequently do your technical processes change/improve? (check most appropriate)

$$
O<3 \text { years } \bigcirc 3-10 \text { years } O>10 \text { years } O \text { Don't know }
$$

4) How frequently does your company or department change its performance measurement system? (select one)

$$
\bigcirc<3 \text { years } \bigcirc 3-10 \text { years } \bigcirc>10 \text { years } \bigcirc \text { Don't know }
$$

5) What is your position in the company? (select one)

Executive OVP O Front line Supervisor O Other (please specify)

If you selected other, please specify:

6) In which department do you work? (select one)

O corporate OHR OFinance OMarketing Oproduction O Engineering OIT O Other (please specify)

If you selected other, please specify: 

7) Estimate the Number of Financial Measures you use to monitor performance, or which are used to measure your performance. (see following sample
list to help estimate number of measures used)

Sales; Capital Expenditures; Maintenance Expenditures; Operating Expenses; SG\&A Expenses; Product Quality Costs (warranty costs); ROI; ROA; Total Manufacturing Costs; Labor Costs; Material Costs; Indirect (overhead) Costs; Manufacturing Process Improvement Costs...

8) Estimate the Number of Internal Operating (Non-Financial) Measures you use to monitor performance, or which are used to measure your performance. (see following sample list to help estimate number of measures used)

Information Technology (\% cost); New Product \% of Sales; Proprietary Products \% of Sales; New Product Introduction vs Competitors; Manufacturing Process Capabilities; Time to Develop Next Generation of Products; Product quality (defect rates); Delivery (on time); Manufacturing Efficiency; Suppliers (quality, defect rates, overtime); Machine Productivity (hours running, available, downtime); Material Usage (inefficiency, waste); Setup Efficiency (setup time, number of setups); Manufacturing Cycle Time (total process time); Inventory (turnover); Product Defects (number of errors, rework, scrap)...

9) Estimate the Number of Employee Related (Learning and Growth, Non-Financial) Measures you use to monitor performance, or which are used to measure your performance. (see following sample list to help estimate number of measures used)

Safety (number of accidents, injuries); Employee Satisfaction (surveys, grievances); Employee Skills (level of education, experience); Employee Empowerment (\# suggestions, \# on improvement teams); Employee Training/Education (hours or time allocated for training); Employee Loyalty / Turnover; Absenteeism; Employee Perception of Leadership...

10) Estimate the Number of Customer (Non-Financial) Measures you use to monitor performance, or which are used to measure your performance. (see following sample list to help estimate the number of measures used)

Customer Acquisition (\# new, \% sales from new); Customer Retention / Loyalty (\# repeat customers); Customer Satisfaction (surveys, complaints); Phone System Utility

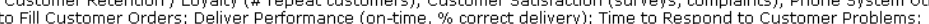
Flexibility/Responsiveness (ability to vary product)...

11) Rank in order from most important to least important the process types related to performance measures you use or by which your performance is measured.

\begin{tabular}{l|l|} 
1) Choose One & v \\
2) Choose One & v \\
3) Choose One & v \\
4) Choose One
\end{tabular}

12) Comments or suggestions?

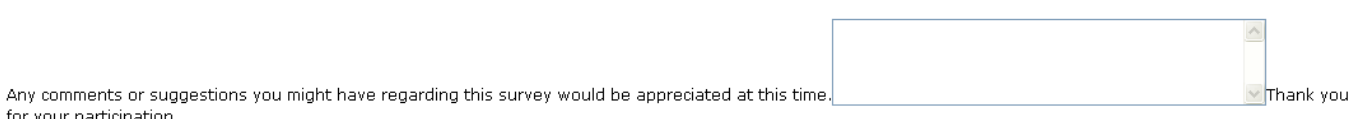
for your participation.

By clicking the Submit Survey button below I acknowledge that I have read the consent form and agree to participate in this study.

Submit Survey

Online Survey Software powered by Vovici.

Note: Question 11 offers the following options presented in random sequence:

Rank in order from most important to least important the process types related to performance measures you use or by which your performance is measured.

$\ll$

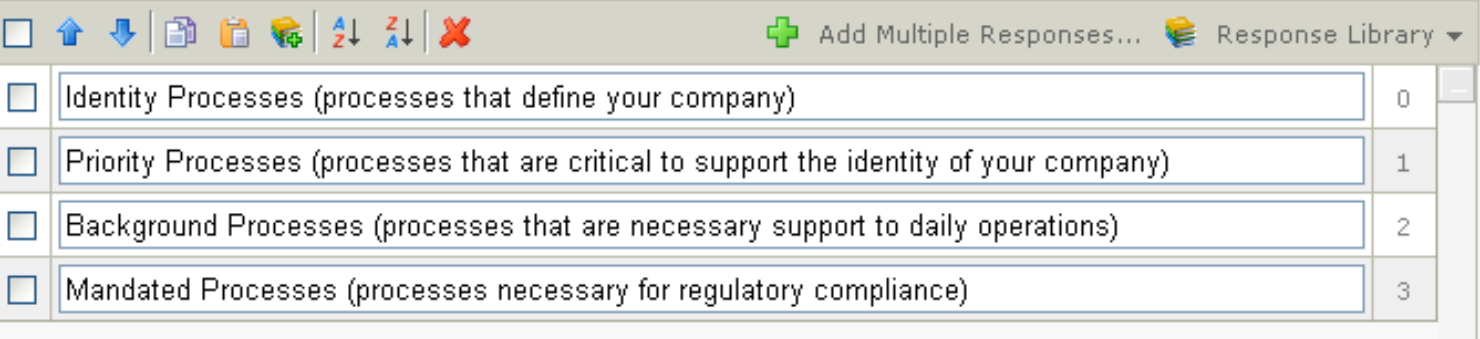

